\title{
The snowball effect in aortic valve disease
}

Citation for published version (APA):

Peeters, F. E. C. M. (2019). The snowball effect in aortic valve disease: gaining insight in imaging, circulating and tissue biomarkers towards a halt in disease progression. [Doctoral Thesis, Maastricht University]. Maastricht University. https://doi.org/10.26481/dis.20190418fp

Document status and date:

Published: 01/01/2019

DOI:

10.26481/dis.20190418fp

Document Version:

Publisher's PDF, also known as Version of record

\section{Please check the document version of this publication:}

- A submitted manuscript is the version of the article upon submission and before peer-review. There can be important differences between the submitted version and the official published version of record.

People interested in the research are advised to contact the author for the final version of the publication, or visit the DOI to the publisher's website.

- The final author version and the galley proof are versions of the publication after peer review.

- The final published version features the final layout of the paper including the volume, issue and page numbers.

Link to publication

\footnotetext{
General rights rights.

- You may freely distribute the URL identifying the publication in the public portal. please follow below link for the End User Agreement:

www.umlib.nl/taverne-license

Take down policy

If you believe that this document breaches copyright please contact us at:

repository@maastrichtuniversity.nl

providing details and we will investigate your claim.
}

Copyright and moral rights for the publications made accessible in the public portal are retained by the authors and/or other copyright owners and it is a condition of accessing publications that users recognise and abide by the legal requirements associated with these

- Users may download and print one copy of any publication from the public portal for the purpose of private study or research.

- You may not further distribute the material or use it for any profit-making activity or commercial gain

If the publication is distributed under the terms of Article $25 \mathrm{fa}$ of the Dutch Copyright Act, indicated by the "Taverne" license above, 


\section{The snowball effect in aortic valve disease}

Gaining insight in imaging, circulating and tissue biomarkers towards a halt in disease progression

\section{PROEFSCHRIFT}

Ter verkrijging van de graad van doctor aan de Universiteit Maastricht, op gezag van de Rector Magnificus, Prof. dr. Rianne M. Letschert volgens het besluit van het College van Decanen, in het openbaar te verdedigen op $\mathbf{1 8}$ april 2019 om $\mathbf{1 0 . 0 0}$ uur

Door

Frederique Elisabeth Corline Maria Peeters 
Prof. dr. H.J.G.M. Crijns

Prof. dr L.J. Schurgers

\section{Copromotores}

Dr. S.J.R. Meex

Dr. B.L.J.H. Kietselaer (Zuyderland Medisch Centrum)

Assessment committee

Prof. dr. T.M. Hackeng (chairman)

Prof. dr. M.W. de Haan

Prof. dr. A.W.J. van 't Hof

Prof. dr. T. Leiner (UMC Utrecht)

Prof. dr. D.E. Newby (University of Edinburgh, Scotland, United Kingdom)

Financial support by the Dutch Heart Foundation for the publication of this thesis is gratefully acknowledged.

Financial support for the printing of this thesis as provided by the following sponsors is gratefully acknowledged:

Stichting Hartsvrienden RESCAR | Bayer | Boehringer-Ingelheim |

ISBN:

978-94-6323-490-0

\section{Graphic Design and layout by:}

M. Mafi Rad

Printed by:

Gildeprint 
Bicuspid aortic valve stenosis and the effect of vitamin $\mathrm{K} 2$ on calcification using ${ }^{18} \mathrm{~F}$-sodium fluoride positron emission tomography/magnetic resonance: the BASIK2 rationale and trial design

NUTRIENTS

2018 MAR 21: 10(4)

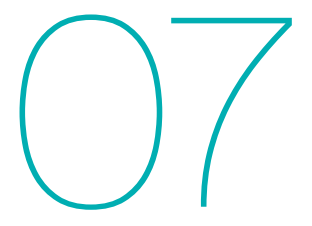

Sex-related differences in valvular fibrosis and calcification in aortic stenosis: application of a noninvasive imaging strategy using ${ }^{18} \mathrm{~F}-\mathrm{NaF}$ PET/CT

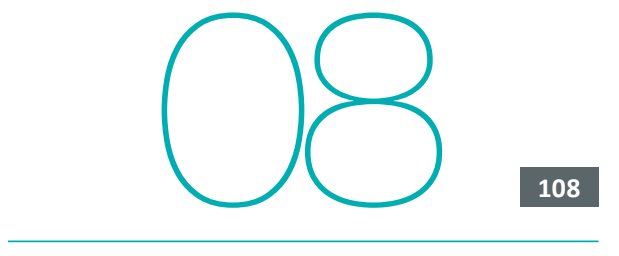

Biomarkers associated with early aortic valve calcification: should we focus on sex specific processes?

SUBMITTED

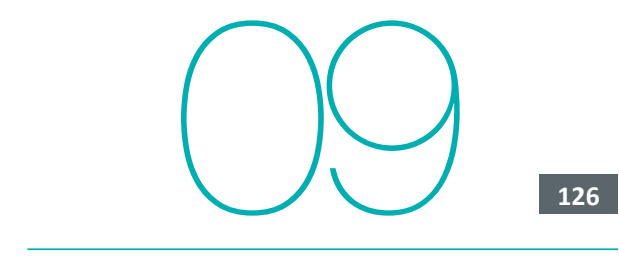

Biological variation of cardiac markers in patients with aortic valve stenosis

SUBMITTED

IN PREPARATION

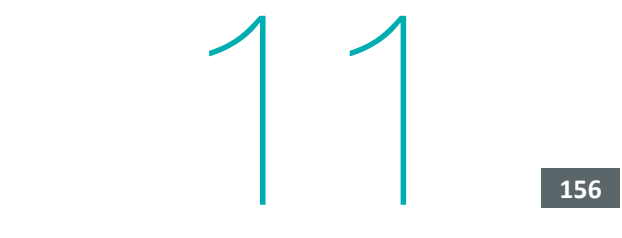

General Discussion

Clinical and echocardiographic determinants in bicuspid aortic dilatation: results from a longitudinal observational study

MEDICINE

2016 DEC;95(52):e5699

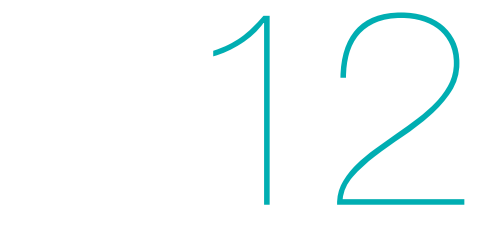

Summary

Nederlandse samenvatting

Valorization

Dankwoord

About the Author

List of publications

IN PREPARATION 
General introduction 


\section{AORTIC VALVE DISEASE: DEFINITION AND EPIDEMIOLOGY}

Aortic valve disease, also known as calcific aortic valve disease (CAVD), calcific aortic valve stenosis (CAVS) or aortic valve stenosis (AS), is a spectrum of disease, ranging from aortic valve sclerosis to severe AS. Aortic valve sclerosis is defined as diffuse thickening of the aortic valve without significant blood flow obstruction. The occurrence of aortic valve sclerosis is common, even in relatively young populations: its incidence increases from $1.9 \%$ to $8.8 \%$ with age and its prevalence is approximately $40 \%$ in patients $>75$ years. Moreover, aortic valve sclerosis is associated with increased cardiovascular risk. ${ }^{1,2}$ Over time, aortic valve disease progresses slowly, and $\sim 2 \%$ of patients develop hemodynamically significant AS per year., ${ }^{3,4}$ Aortic valve stenosis is defined as narrowing of the valve causing blood flow obstruction. It is relatively uncommon in patients $<65$ years old without a congenital valve abnormality. However, the Troms $\varnothing$ study reported a substantial age-dependent increase in prevalence: $0.2 \%$ in $50-59$ years, $1.3 \%$ in $60-69$ years, $3.9 \%$ in 70-79 years and $9.8 \%$ in 80-89 years. The incidence of new AS was 5 per 1000 per year and progression rates were variable. ${ }^{5}$ Upon the development of symptomatic severe AS, the prognosis without intervention is dismal and aortic valve replacement (AVR), or transcatheter aortic valve implantation (TAVI) is indicated. In patients $>70$ years of age with tricuspid aortic valve stenosis, the need for intervention becomes more common. In younger patient groups, development of significant AS is mostly seen in patients with a bicuspid aortic valve (BAV). ${ }^{6}$ BAV is a common congenital abnormality, occurring in 13 per 1000 people in the general population. ${ }^{78}$ It is associated with valvular and vascular morbidity, and development of AS at a young age is common. In this patient group, valve replacement is usually indicated between the fourth and sixth decade 6,9

With an increasingly elderly population, disease burden of AS will increase in the coming years. The number of patients with an indication for treatment is expected to double by 2050 in Europe and the USA. ${ }^{10}$ Due to complexity and challenges, the need for long-term follow-up, investigation and treatment costs result in a substantial impact of AS (and other valvular heart diseases) on the healthcare systems, showing a trend to increase even more with the aging population.

\section{PATHOPHYSIOLOGY: INITIATION AND DISEASE PROGRESSION}

Whereas AS was considered a passive disease whereby 'wear and tear' resulted in calcification of the valve, emerging evidence showed that it is an active disease, involving highly complex and tightly regulated pathways. ${ }^{11,12}$ However, there is still lack of understanding of the pathophysiological processes and their exact contribution to AS and its progression. Roughly, two phases in AS can be distinguished: initiation and progression, each with common and different dominating processes. The initiation phase shares similarities to atherosclerosis and is dominated by endothelial activation and damage, lipid infiltration and oxidation and inflammatory response. Within affected regions, microcalcifications colocalize with lipids, creating a nidus for calcium crystal deposition and hydroxyapatite formation. Moreover, the initiation phase of AS shows common risk factors including age, male sex, body mass index, smoking, hypertension and elevated lipid levels. ${ }^{13,14}$ Whereas inflammation is predominant in the initiation phase, fibrosis and calcification are becoming increasingly dominant in the progression phase. Alterations in nitric oxide expression, up-regulation of the renin-angiotensin system (RAS), upregulation of pro-calcific regulatory pathways (Notch, receptor activator of nuclear factor kappa B (RANK)/receptor activator of nuclear factor B ligand (RANKL)/osteoprotegerin (OPG), WBT/b-catenin and bone morphogenetic proteins) and downregulation of anti-calcification processes (Fetuin-A, Matrix Gla Protein) are key components in the propagation phase. ${ }^{11,15-18} \mathrm{As}$ a consequence, valvular dysfunction and changes in mechanical stress and flow are caused, thereby creating self-sustaining mechanisms underlying progression.

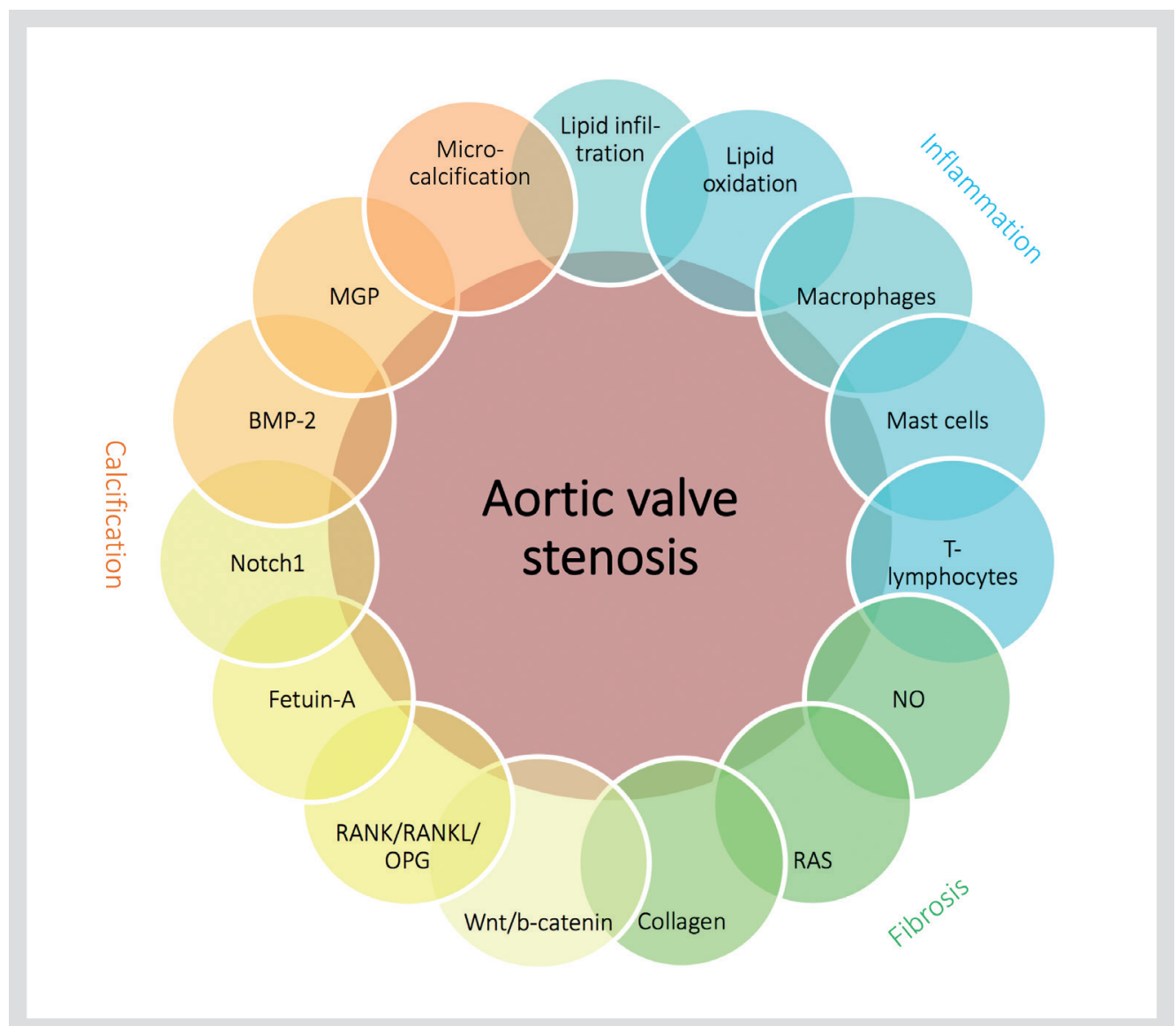

Figure 1. Overview mechanisms aortic valve stenosis. Inflammatory mechanisms in blue, fibrosis in green and calcification in orange and yellow.

Abbreviations: BMP: Bone Morphogenetic Protein-2; MGP: Matrix Gla Protein; NO: Nitric Oxide: RANK/ RANKL/OPG: nuclear factor kappa B (RANK)/receptor activator of nuclear factor B ligand (RANKL)/osteoprotegerin (OPG); RAS: Renin-Angiotensin System

\section{PATIENT MONITORING - IMAGING}

AS is typically diagnosed by echocardiography after detection of a systolic murmur. ${ }^{19}$ That is, echocardiography is the principal diagnostic tool in clinical practice to determine the presence of aortic valve stenosis and the degree of severity. Moreover, it is widely available, safe and provides clinicians with information regarding other valvular disease and ventricular (dys)function and remodeling. ${ }^{20}$ The international guidelines recommend grading of AS severity by integrating measurements at the valvular level (peak velocity, mean gradient, (indexed) aortic valve area and velocity ratio). ${ }^{21}$ However, due to technical limitations inherent to the echocardiographic examination, guidelines recommend to consider not only echocardiographic parameters (including ventricular dimensions and function), but rely on clinical evaluation (including symptoms, physical examination, and other diagnostic testing) besides estimating AS severity. ${ }^{20-23}$ 
Since AS progression shows a marked variability between patients 5 , follow-up assessment of AS by echocardiography should be performed at regular intervals, depending on severity and rate of progression of AS. ${ }^{23}$ Unfortunately, discordant echocardiographic measurements are found in a substantial group of patients with AS, challenging a correct interpretation of AS severity.

In asymptomatic patients with potential severe AS and conflicting measurements on echocardiography, quantification of aortic valve calcification (AVC) using computed tomography (CT) is recommended by current guidelines. AVC scoring is closely associated with hemodynamic measurements on echocardiography. Moreover, it may be used for prediction of disease progression and holds potential as an alternative to assess AS severity. ${ }^{24-27}$

Although current assessment and management of AS relies heavily on echocardiographic measurements, other imaging modalities such as positron emission tomography (PET) and magnetic resonance imaging (MRI) hold potential as alternative approaches providing the clinician additional information regarding disease progression and left ventricular fibrosis and decompensation. ${ }^{26}$

\section{PROGRESSIVE AS - TREATMENT}

Upon mild valve obstruction, disease progression with increasing hemodynamic severity is common. Once patients develop symptomatic severe AS prognosis without intervention is disma: mortality is estimated at 50\% in 3-5 years. ${ }^{28,29}$ Despite growing knowledge, experience, and technological developments, the only treatment for (symptomatic) severe CAVS is surgical aortic valve replacement (AVR) or transcatheter aortic valve implantation (TAVI). Currently, the choice of intervention type depends on patient comorbidities and risk of surgery, risk scores, and local experience and is carefully assessed by a Heart Team. TAVI is mostly recommended in patients $>75$ years of age and patients with high-risk, whilst in low-risk patients or younger patients, AVR (mechanical or bioprosthetic valve) is preferred. ${ }^{20}$ Mortality associated with AVR decreased dramatically in the last decades and intervention improves symptoms and increases life expectancy in patients with severe symptomatic AS. ${ }^{30,31}$ Moreover, TAVI shows favorable results over surger in elderly patients with an increased surgical risk. For patients with asymptomatic severe AS, are controversial, representing an unmet clinical need to develop new treatment strategies delaying

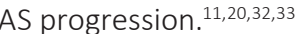

Overall, the timing of intervention is challenging. One of the indications for valve interventions is the development of symptoms. Assessment of symptoms in patients with multiple comorbidities can be challenging though, and exercise testing is recommended to unmask symptoms. Another indication is evidence of left ventricular systolic dysfunction (LVEF $<50 \%$ ), a feature that often occurs in a late stage of disease, and tends to be irreversible. Intervention should be considered in asymptomatic patients with fast progression, severe pulmonary hypertension and with "marked elevated brain natriuretic protein (BNP) levels confirmed by repeated measurements" 20,34-37 Strikingly, the role of circulating biomarkers in AS is limited, while they are commonly used in clinical decision making for diagnosing, risk stratification and management of other cardiovascular diseases. ${ }^{38,39}$ However, recent studies suggest that biomarkers have a prognostic value in AS and acknowledge a potential role for biomarkers as a complementary approach to gain insight in As progression and timing of intervention. ${ }^{40-43}$ These studies need to be validated in larger cohorts.
SEX DIFFERENCES IN AS

alcification, inflammation and fibrosis are key players in the progression of AS. ${ }^{11,33,44,45}$ Howeve sex differences may apply. ${ }^{46}$ Aortic valves of women with severe AS show less aortic valve calcification (AVC) on CT when compared to men with similar hemodynamic severity of AS, but similar progression rates were found in males and females..$^{47-50}$ Recently, it was hypothesized that more valvular fibrosis might explain the basis of this sex-related discrepancy between the AVC load and hemodynamic severity in females..$^{51}$ This could be of importance in the understanding of AS pathophysiology and the development of sex-specific drug therapeutic options and therefore, in clinical practice.

\section{GAPS IN KNOWLEDGE}

Prediction of progression of aortic stenosis and timing of surgery remains challenging. Further optimization and integration of imaging and circulating biomarkers seems reasonable and prompts questions like: Can the integration of a multimodality imaging approach help clinicians in optimizing assessment of AS severity and predict progression? Also, will circulating biomarkers play a role in follow-up and timing of surgery? At present, there is no consensus on effective pharmacological interventions to halt AS progression. In addition, we are unaware of how sex-related differences in predominant processes may help clarify disease progression and how these differences are affected by pharmacological therapy. Therefore, additional questions can be asked, such as: Can we develop treatment strategies to stop the "snowball effect" of AS? And, should we focus on tailor-made management of AS, also considering differences between males and females?

\section{AIM AND OUTLINE OF THE THESIS}

This thesis aims to increase knowledge of pathophysiology, imaging and blood biomarkers and potential treatment options in AS.

In Chapter 2, we start our investigations by discussing current and evolving knowledge of the pathophysiology of AS. Moreover, we provide insight into the challenges we are facing in daily practice to develop an effective medical treatment strategy to reduce or halt disease progression. Finally, we provide a rationale that targeting Matrix Gla Protein (MGP; a vitamin K dependent protein known as a vascular calcification inhibitor) holds potential in the management of AS, and propose the application of additional imaging techniques to visualize AS and progression.

If MGP plays a vital role in the inhibition of valvular and vascular calcification, depletion of Vitamin K, thereby losing MGP functionality, should theoretically enhance calcification. Chapter 3 investigates whether an association between calcification and treatment with Vitamin $K$ Antagonists (VKA) exists in a propensity-score matched population treated with VKA, non-Vitamin K Antagonist Coagulants (NOAC) and patients not treated with oral anticoagulants.

In Chapter 4 and 5, two studies investigate whether progression of aortic valve stenosis can be reduced by increasing the active form of MGP through supplementation with vitamin K1 (Chapter 4) and vitamin K2 (Chapter 5). More specifically, Chapter 4 investigates whether supplementation with vitamin K1 in a group of in patients with tricuspid aortic valve stenosis results in a reduction in progression. Additionally, Chapter 5 describes the rationale and design of a study investigating the effect of vitamin $\mathrm{K} 2$ on progression in patients with bicuspid AS.

Chapter 6 investigates in valvular histology whether the inactive form of MGP is present in regions contiguous to calcified regions in the valve, thereby suggesting it is a forerunner for development and expansion of calcification. 
Chapter 7 places particular focus on the application of the specific imaging technique PET/CT using ${ }^{18} \mathrm{~F}$-sodium fluoride to describe calcification, calcification activity and fibrosis in aortic valve stenosis. Moreover, it investigates potential phenotype differences in females and males with aortic valve stenosis.

In Chapter 8, potential sex-specific processes leading to a different phenotype (fibrotic vs. calcific) are investigated in a patient group with valve calcification without hemodynamic consequences, using a panel of circulating biomarkers. Subsequently, in Chapter 9 serial measurements of a specific set of cardiac biomarkers were studied in a group of patients with stable aortic valve stenosis; what is their natural variation and do they hold potential to be integrated into clinical follow-up?

AS mostly develops in patients with a tricuspid aortic valve over 65 years of age. The occurrence of aortic valve stenosis at younger ages is most commonly seen in patients with a bicuspid aortic valve. Early development of valvular and vascular complications is typical in this group. In Chapter 10, the association between echocardiographic and clinical parameters and aortic dilatation in patients with a bicuspid aortic valve was investigated.

The final chapter, Chapter 11, contains a general discussion of the results described in this thesis and provides directions for future research.
REFERENCES

Corfey S, Cairns BJ, lung B. The modern epidemiology of 18. Koos R, Brandenburg V, Mahnken AH et al. Association of . Heart 2016;102:75-85 of Cardiology 2014;63:2852-61. calcification determinants and progression in the poplat 2007;27:642-8.

4. Novaro GM, Katz R, Aviles RJ et al. Clinical factors, but not $C$-reactive protein, predict progression of calcific aortic-valve disease. the Cardiovascular Health Study. Journ

5. Eveborn $G W$ Schirmer $H$, Heggelund $G$, Lunde $P$, Rasm sen $\mathrm{K}$. The evolving epidemiology of valvular aortic stenosis. the Tromso study. Heart 2013;99:396-400.

6. Roberts WC, Ko JM. Frequency by decades of unicuspid, bicuspid, and tricuspid aortic valves in adults having isoated aortic valve replacement for aortic stenosis, with or 2005:111:920-5. of the American College of Cardiology 2010;55:2789-800. Writing Group M, Mozaffarian D, Benjamin EJ et al. Heart Disease and Stroke Statistics-2016 Update: A Report 2016;133:e38-360.

9. Michelena HII Dejardins VA Avierinos IF et al Naturat history of asymptomatic patients with normally functioning minimally dysfunctional bicuspid aortic valve in the community. Circulation 2008;117:2776-84.

10. Osnabrugge RL, Mylotte D, Head SJ et al. Aortic stenosis in the elderly: disease prevalence and number of candidates and modeling study Journal of the American Coll Cardiology 2013;62:1002-12.

11. Pawade TA, Newby DE, Dweck MR. Calcification in Aortic Stenosis: The Skeleton Key. Journal of the American College of Cardiology 2015;66:561-77.

2. Rajamannan NM, Evans $\mathrm{FJ}$, Aikawa E et al. Calcific aortic and and Blood Institute Aortic Stenosis Working Group. Executive summary: Calcific aortic valve disease-2011 update. Circulation 2011;124:1783-91

13. New SE, Aikawa E. Molecular imaging insights into early inflammatory stages of arterial and aortic val

- Stewart BF, Siscovick D, Lind BK et a C Clinical foctors associated with calcific aortic valve disease. Cardiovascular Health Study. Journal of the American College of Cardioogy 1997;29:630-4.

15. El Accaoui RN, Gould ST, Hajj GP et al. Aortic valve sclerosis mice deficient in endothellal exide synthase. Amer2014:306:H1302-13.

Nays in aortic value cells . cardiology 2009;47:828-34.

17. Schurgers LJ, Vitto J, Reutelingsperger CP. Vitami K-dependent carboxylation of matrix Gla-protein: a crucia lar medicine 2013;19:217-26.
fetwin-A Bevels with the progression of alartic valve calcification in non-dialyzed pagients. European heart journ 2009;30:2054-61.

. Lindman BR, Bonow RO, Otto CM. Current management of calcific aortic stenosis. Circulation research 2013;113:223-37. lines for the m, Falk V, BaxJJ et al. 2017 ESC/EACTS Guide pean heart journal 2017:38:2739-2791.

on the Echocardiographic Assessment of Aortic Valve Stenosis: A Focused Update from the European Association of Cardiovascular Imaging and the American Society of Echodiography: official publication of the American Society of Echocardiography 2017;30:372-392.

Nishimura RA, Otto CM, Bonow RO et al. 2017 AHA/ACC Focused Update of the 2014 AHA/ACC Guideline for the Management of Patients With Valvular Heart Disease: Report of the American College of Cardiology/America Lines Circulation 2017,135 e1150 C1195. 作 Heart Disease: a report of the American College of Cardioogy/American Heart Association Task Force on Practice Budeff ML Takasu L, Katz, R et al. Reproduct

measurements of a katic value calcification, mitral ann calcification, and aortic wall calcification in the multi-eth 2006;13:166-72.

Cartlidge TR, Pawade TA, Dweck MR. Aortic stenosis and C calcium scoring. Is it for everyone? Heart 2017;103:8-9.

tion in Patients With Aortic Stenosis Using Nove ImacApproaches. Circulation Cardiovascular imaging 2015;8:e00342

Nguyen V, Cimadevilac, Estellat $C$ et al. Haemodynamic and anatomic progression of aortic stenosis. Heat 2015;101:943-7. population: treatment considerations and sur. Annals of thoracic surgery 2010;89:453-8.

Pellika PA, Sarano ME, Nishimura RA et al. Outcome of 622 adurts with asymptomatic, hemodynamically significant 2005;111:3290-5.

JM, O'Brien SM, Wu C, Sikora JA, Griffith BP, Gammie IS. Isolated aortic valve replacement in North America comprising 108,687 patients in 10 years: changes in risks, valve types, and outcomes in the Society of Thoracic Surdiovascular surgery 2009;137:82-90.

utcome trends for nnals of thoracic sur-

. Hutcheson JD, Aikawa E, Merryman WD. Potential drug tarology 2014;11:218-31.

. Peeters F, Meex SJR, Dweck MR et al. Calcific aortic valve approach towards diagnosis and treatment. Europer heart journal 2017.
C Ratcliffe $M$ et al. Severe aortic stenosis 
34. Everett RJ, Newby DE, Jabbour A, Fayad ZA, Dweck MR. The Role of Imaging in Aortic Valve Disease. Current cardiova Connolly HM Oh JK Orszulak TA

ment for artic stenosis with seval. Aortic valve replace function. Prognostic indicators. Circulation 1997;95:2395 400.

36. Connolly HM, Oh JK, Schaff HV et al. Severe aortic stenosis with low transvalvular gradient and severe left ventricular dystunctions

Lancellotit P. Donal E, Magne $\rfloor$ et al.

asymptomatic moderate to severe aortic stenosis: the importance of the valvular, arterial and ventricular interplay. Heart 2010;96:1364-71

8. Toutouzas K, Stathogiannis K, Latsios $G$ et al. Biomarkers in scatheter aortic valve implantation. Current medicina

van Holten TC, Waanders LF, de Groot PG et al. Circulatin biomarkers for predicting cardiovascular disease risk; a systematic review and comprehensive overview of meta-ana yses. Plos one 2013,8.e62080.

et al. High-sensitivity troponin I concentrations are a marker of an advanced hyperaortic stenosis. European heart journal 2014;35:2312-21.

1. Dahou A, Clavel MA, Capoulade R et al. B-Type Natriuretic Peptide and High-Sensitivity Cardiac Troponin for Risk Stratification in Low Flow, Low Gradent Aortic Stenosis: A 2018;11:939-947.

42. Dweck MR, Everett RJ. Multibiomarker Strategies in Aortic
Stenosis JACC Cardiovascular imaging 2018;11:948-950. Stenosis. JACC Cardiovascular imaging 2018;11:948-950. Lindman $B R$, Breyley $J G$, Schilling J et al. Prognostic utility
of novel biomarkers of cardiovascular stress in patients with aortic stenosis undergoing valve replacement. Hea

44. Carabello BA, Paulus WJ. Aortic stenosis. Lancet

5. Otto CM, Kuusisto J, Reichenbach DD, Gown AM, O'Brien $\mathrm{KD}$. Characterization of the early lesion of 'degenerative' valvular aortic stenosis. Histological and immunohisto-

.

differences in calcific aortic valve stenosis: pathophysiology, epidemiology, etiolHeart 2017.

47. Aggarwal SR, Clavel MA, Messika-Zeitoun D et al. Sex differ ences in aortic valve calcification measured by multidetec-

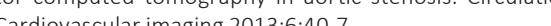

Clavel MA, Messika-Zeitoun D, Pibarot P et al. The complex
nature of discordant severe calcified aortic valve disease grading: new insights from combined Doppler echocardiographic and computed tomographic study. Journal of the

49. Clavel MA, Pibarot $P$, Messika-Zeitoun $D$ et al. Impact of in patients with aortic stenosis: results of an internation registry study. Journal of the American College of Cardio-

ogy 2014;64:1202-13.
50. Thomassen HK, Cioffi G, Gerdts E et al. Echocardiographic aortic valve calcification and outcomes in women and $\mathrm{men}$
Between Aortic Valve Calcification and Hemodynamic (1) 


\section{Calcific aortic valve stenosis: hard disease in the heart. A biomolecular approach towards diagnosis and treatment}

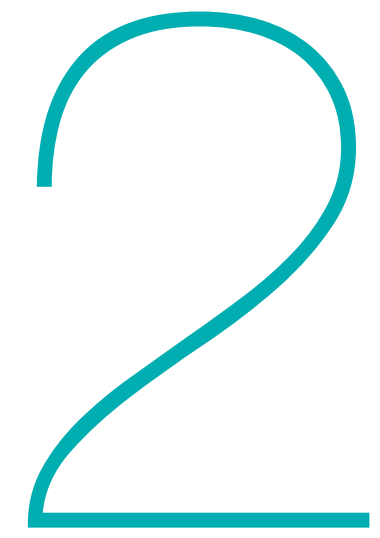

Frederique E.C.M. Peeters ${ }^{1}$, Steven J.R. Meex², Marc R. Dweck MD³, Elena Aikawa4, Harry J.G.M. Crijns ${ }^{1}$, Leon J. Schurgers $5^{*}$, Bas L.J.H. Kietselaer ${ }^{6^{*}}$

${ }^{1}$ Maastricht University Medical Center+ and CARIM, department of Cardiology, Maastricht, the Netherlands,

${ }^{2}$ Maastricht University Medical Center+ and CARIM, department of Clinical chemistry, Maastricht, the Netherlands,

${ }^{3}$ Centre for Cardiovascular Science, University of Edinburgh, Edinburgh, UK,

${ }^{4}$ Harvard Medical School, department of Medicine, Cardiovascular Division, Brigham and Women's Hospital, USA,

${ }^{5}$ Maastricht University and CARIM, department of Biochemistry, Maastricht, the Netherlands,

${ }^{6}$ Zuyderland Medisch Centrum Heerlen/Sittard, Heerlen/Sittard, the Netherlands,

*contributed equally 


\section{ABSTRACT}

Calcific aortic valve stenosis (CAVS) is common in the aging population, and set to become an increasing economic and health burden. Once present, it inevitably progresses and has a poor prognosis in symptomatic patients. No medical therapies are proven to be effective in holding or reducing disease progression. Therefore aortic valve replacement remains the only available treatment option. Improved knowledge of the mechanisms underlying disease progression has provided us with insights that CAVS is not a passive disease. Rather, CAVS is regulated by numerous mechanisms with a key role for calcification. Aortic valve calcification (AVC) is actively regulated involving cellular and humoral factors that may offer targets for diagnosis and intervention. The discovery that the vitamin K-dependent proteins are involved in the inhibition of AVC has boosted our mechanistic understanding of this process and has opened up novel avenues in disease exploration. This review discusses processes involved in CAVS progression, with an emphasis on recent insights into calcification, methods for imaging calcification activity and potential therapeutic options.

\section{INTRODUCTION}

Degenerative calcific aortic valve stenosis (CAVS) is the most common type of valvular disease in the Western world, representing a substantial and increasing disease burden in the aging population. ${ }^{1}$ Upon mild valve obstruction, disease progression with increasing hemodynamic severity is inevitable. Once symptomatic severe CAVS has developed, the prognosis without intervention is dismal. Despite growing knowledge, experience and technological developments, the only treatment for (symptomatic) severe CAVS is surgical or transcatheter aortic valve replacement (AVR), to which not all patients are suited. ${ }^{2}$ Pharmacological interventions have thus far failed to alter the course of CAVS. Therefore, an unmet clinical need exists to develop new treatment strategies delaying CAVS progression.

We still lack precise molecular insight in to the pathophysiological underlying CAVS, although calcification is well known to play a fundamental role in progressive valvular narrowing. Today calcification is no longer considered a passive consequence of ageing, but rather an active proces involving cellular and molecular pathways. The exact processes underlying the initiation and progression of valvular calcification remain unresolved. ${ }^{3}$ Understanding the biomolecular mechanisms related to the genesis of calcification in CAVS will propel our knowledge and open novel avenues for diagnosis and treatment. In this review, we summarize the latest research progress in the pathophysiology of CAVS and offer novel targets holding potential for pharmacological interventions and imaging.

\section{AORTIC VALVE CUSP FUNCTION}

ortic valve cusps (or leaflets) must be both strong and flexible to withstand the considerable mechanical stress and strain associated with valve closure. To maintain cusp function, the specialized cusp microarchitecture is crucial and consists of three layers: fibrosa, spongiosa and ventricularis (Figure 1). ${ }^{4}$ Valvular endothelial cells (VECS) are located at valvular blood-contacting surfaces, constituting a barrier that regulates valve permeability, the adhesion of inflammatory cells and paracrine signalling. Valvular interstitial cells (VICS), the major cell type, are present throughout all valvular layers. VICs are key in valve remodelling, regulating both the synthesis and degradation of extracellular matrix components. Physiologically, VICs exist in a quiescent state,

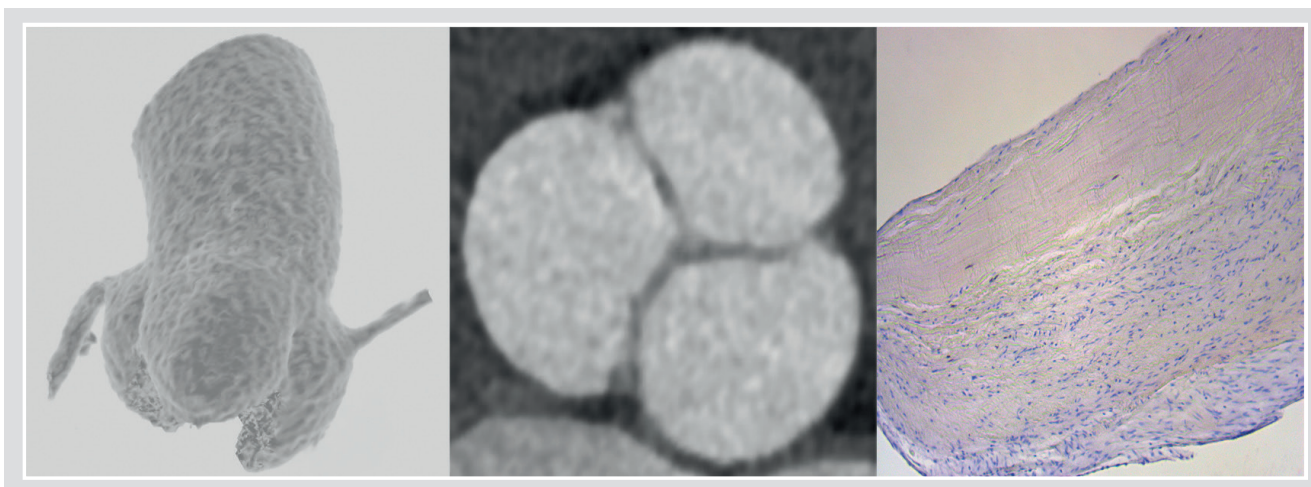

Figure 1. Aortic valve. Left panel: 3D-reconstruction (from bottom to top:) aortic valve with 3 cusps and proximal ascending aorta. Middle panel: $2 \mathrm{D}$ view. Right panel: valvular histology (bottom to top): ventricularis, spongiosa, fibrosa 
with similar characteristics to fibroblasts ${ }^{5}$ Stimulation of VECs and VICs by molecular and mechanical triggers including high blood pressure, altered shear stress, cytokines, and growth-factor contributes to CAVS pathophysiology, altering the local valve environment and making it calcification prone.

\section{CAVS ETIOLOGY}

Whilst the most common cause of aortic stenosis in the western world is degenerative CAVS (referred to as "CAVS" in this review), rheumatic heart disease remains common in developing countries. Rheumatic aortic stenosis is caused by an abnormal immune response to group A streptococcal infections. Calcification is again a predominant feature, and whilst this is believed to relate to chronic inflammation, precise mechanisms remain poorly defined. ${ }^{6}$ CAVS is accelerated in patients with congenitally bicuspid aortic valves (BAV) with aortic stenosis developing several decades earlier than in patients with trileaflet valves. More than $50 \%$ of patients with severe aortic stenosis requiring aortic valve replacement have BAV.?

\section{CAVS PATHOPHYSIOLOGY}

\section{Initiation phase}

CAVS can be divided in two distinct phases; the initiation and propagation phase, each dominated by different mechanisms (Figure 2). The initiation phase shows similarities with atherosclerosis, both ignited by endothelial activation/damage and an inflammatory response ${ }^{8}$ and sharing common risk factors including age, male sex, body mass index, smoking, hypertension and elevated lipid levels including $\mathrm{Lp}(\mathrm{a}) .{ }^{9}$ Moreover, stenotic valves from animals fed a high-fat diet display similar lesions as found in early human atherosclerotic plaques. ${ }^{10,11}$

Classically, the initiation phase is triggered by mechanical stress in the valve causing endothelial damage and activation. This is perhaps best illustrated by the accelerated development of aortic stenosis in patients with BAV that are characterised by altered flow patterns, increased mechanical stress and reduced shear stress. ${ }^{12}$ The endothelial damage results in lipid infiltration and subsequent oxidation, thereby initiating an inflammatory response within the valvular endothelium involving macrophages, T-lymphocytes, and mast cells. ${ }^{11}$ Within affected regions, microcalcifications colocalize with lipids. Formation of microcalcifications is mediated by release of apoptotic bodies and extracellular vesicles, in a similar manner to vesicle-induced calcification in bone and the vasculature. ${ }^{8,13}$ These calcification-prone extracellular vesicles function as nucleating sites for calcium crystal deposition and facilitate formation of hydroxyapatite. Hydroxyapatite crystals in turn set the stage for CAVS progression by (1) expanding quickly (creating more nucleation sites for calcium deposition) and, (2) evoking additional pro-inflammatory responses, 5,8,14

Propagation phase: fibrosis and calcification as hallmarks of disease progression

Whereas the initiation phase is mainly mediated by inflammatory responses, the role of inflammation and lipid deposition is less prominent in the propagation phase (Figure 2). Instead, it is characterized by fibrosis and accelerated calcification, leading to valvular dysfunction and changes in mechanical stress and flow, thereby creating self-sustaining mechanisms underlyin CAVS progression. Pro-fibrotic processes are mediated by [1] reduced nitric oxide expression following endothelial injury ${ }^{15}$ and [2] upregulation of the renin-angiotensin system (RAS), and formation of angiotensin II (ANGII). Down regulation of expression of the angiotensin II type 2

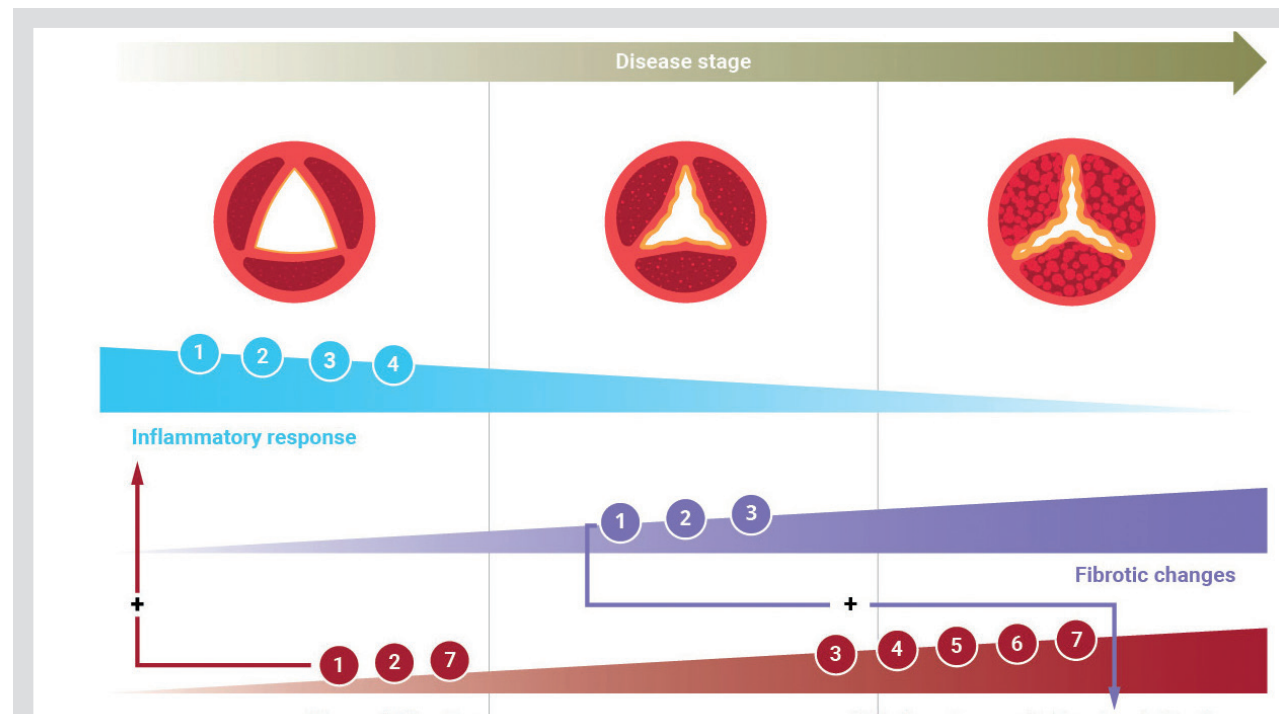

Microcalcification

VIC phenotype switching \& calcification

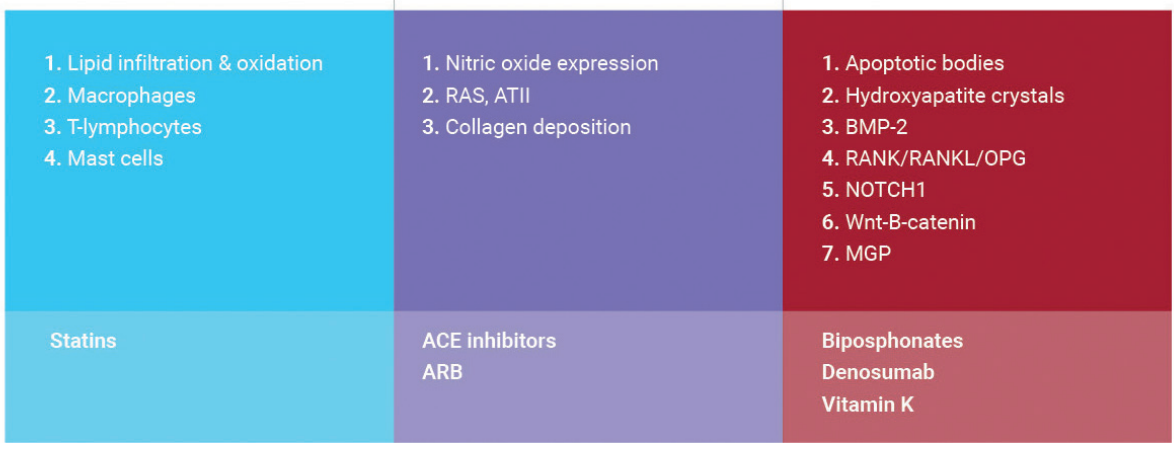

Figure 2. Pathophysiology and potential treatment targets (schematic overview)

A: Progressive CAVS stages from non-stenotic to severe stenosis (left-right). Progressive thickening and calcification result in valvular dysfunction, characterized by decreased cusp mobility and opening altered hemodynamics and stress

B: Cellular involvement in CAVS. Endothelial damage triggers lipid infiltration and upon oxidation an inflammatory response involving macrophages, T-lymphocytes and mast cells. Inflammation triggers phenotypic switching of VICs resulting in increased extracellular vesicle release, providing a nidus for calcification. Microcalcification provokes an inflammatory response, resulting in increased apoptosis and/or delayed phagocytosis thereby expanding calcium deposition. Upon propagation, pro-fibrotic and pro-calcific processes dominate. Pro-fibrotic changes leading to collagen deposition and facilitating progressive calcification are mediated by reduced nitric oxide expression and upregulation of RAS. Calcification is the dominant process driving disease progression. VIC phenotype switching to an osteoblast phenotype is thought to play a role in the progression phase by multiple regulatory pathways including Notch, RANK/RANKL/OPG, Wnt/b-catenin and BMP-2

C: Potential pharmacological interventions 
receptor has been shown to result in a predominant pro-fibrotic profile, resulting in collage deposition and the facilitation of progressive calcification. ${ }^{16}$ CAVS is viewed as a fibrocalcific disease; however once calcification becomes abundant, pro-osteogenic mechanisms become overwhelming, ultimately leading to severe calcification and valvular dysfunction. The phenotypic switching of VICs into an osteoblast-like phenotype is thought to be the fundamental step in accelerating valvular calcification, initiated at least in part by inflammation. In the propagation phase disease progression is driven by calcific regulatory pathways including Notch, receptor activator of nuclear factor kappa B(RANK)/receptor activator of nuclear factor kappa B ligand (RANKL)/osteoprotegerin (OPG), Wnt/b-catenin and bone morphogenetic proteins (BMPs). Notch-1 is essential in the development of the aortic valve during embryology and a mutation in Notch-1 is associated with development of BAV (but multiple genetic factors associated with BAV and CAVS have been described). ${ }^{17}$ Also, Notch-1 is associated with early valve calcification by stimulating BMP-2. ${ }^{18}$ BMP-2 is upregulated through binding of RANKL to RANK. Activation of the RANK/RANKL pathway results in formation of proteins involved in calcification such as alkaline phosphatase and osteocalcin ${ }^{16}$ and is involved in CAVS (Figure 2).

Belonging to the multifunctional TGF- $\beta$ superfamily, BMP-2 is an important osteogenic differentiation factor. BMP-2 is a key protein in phenotypic switching of VICs and hence in the development of aortic valve calcification. ${ }^{16}$ Physiologically, BMP is inhibited by matrix Gla-protein (MGP). ${ }^{19}$ The vital role of MGP to inhibit vascular calcification was demonstrated in MGP-deficien mice, showing lethal rupture of severely calcified arteries $>2$ months after birth. ${ }^{20}$ Although the inhibitory function of MGP on BMP-2 and subsequent VIC differentiation in CAVS seems evident, MGP also exerts its effect via a second mechanism. MGP interacts directly with hydroxyapatite inhibiting growth of hydroxyapatite crystals in vascular tissue. ${ }^{19}$ Since we hypothesize that hydroxyapatite crystals are involved in the early phase of CAVS, MGP is a potential target to inhibit microcalcification. MGP is a vitamin K-dependent protein and is present in two distinct forms; uncarboxylated inactive (UCMGP) and carboxylated active (cMGP). Like all vitamin K-dependent proteins, MGP requires vitamin K-induced carboxylation to exert its function (Figure 3). ${ }^{19}$ VKA inhibits recycling of vitamin $\mathrm{K}$ thereby inducing inactive vitamin $\mathrm{K}$-dependent proteins. Although VKA is important for prophylaxis of thrombo-embolic events in certain patient-populations, calcification should be acknowledged as a side effect. In animal models, warfarin treatment increased vascular and valvular calcification, similar to the MGP knock-out mouse.21 The detrimental effect of warfarin was also identified in humans, where patients using VKA demonstrated more vascular and valvular calcification. ${ }^{22,23}$ With our expanding knowledge of CAVS pathophysiology, possible treatment targets for pharmacological interventions become evident.

\section{PHARMACOLOGICAL TREATMENT TARGETS IN CAVS}

Current guidelines do not recommend pharmacological interventions to halt CAVS progression. However, the importance and need to reduce or even reverse progression of CAVS is evident. Therefore, multiple observational studies and randomized controlled trials (RCTs) have attempted to repurpose commonly used pharmacological interventions to slow CAVS progression.

\section{ACE-inhibitors and ARB}

Hypertension affects the stenotic aortic valve and increases afterload, thereby accelerating LV hypertrophy. Both LV hypertrophy and high valvuloarterial impedance are associated with adverse events in patients with CAVS. ${ }^{24,25}$ Therefore, current guidelines recommend treatment of concomitant hypertension. ${ }^{2}$

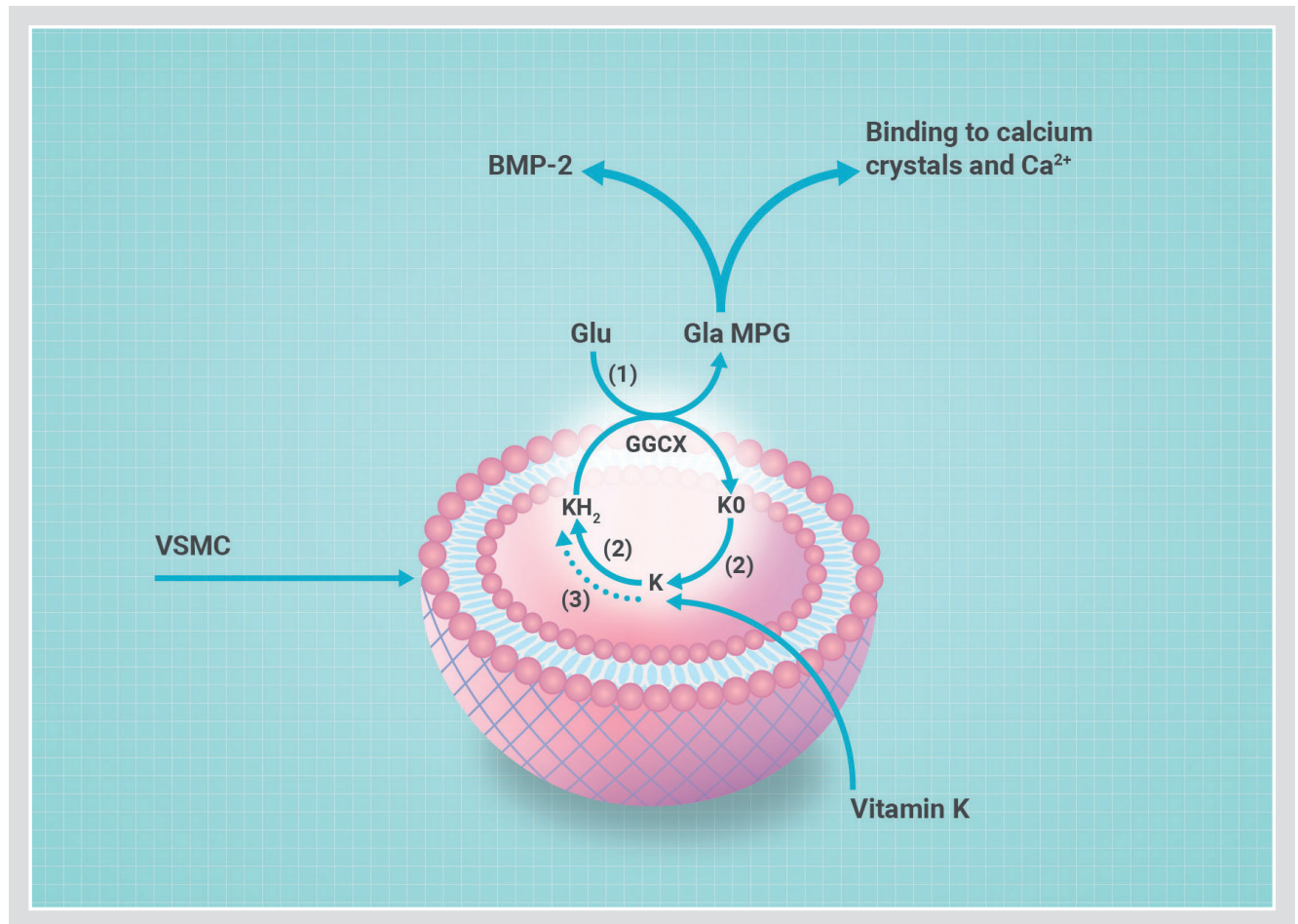

Figure 3. Synthesis of active MGP: schematic overview of vitamin K metabolism. The vitamin K cycle has a central role in the posttranslational carboxylation of glutamate (Glu) to $\gamma$-carboxyglutamate (Gla). Reduction of vitamin $\mathrm{K}$ to vitamin $\mathrm{K}$ hydroxyquinone (KH2) in presence of Vitamin $\mathrm{K}$ Epoxide reductase (VKOR)(2) or DDT-diaphorase(3). KH2 is oxidized during $\mathrm{y}$-glutamyl carboxylation by gamma-glutamyl carboxylase (GGCX)(1) into vitamin K epoxide (KO). KO is reduced to vitamin K by VKOR(2). Carboxylated $M G P(C M G P)$ is active MGP that is secreted in the extracellular environment and inhibits calcification via binding to BMP-2 or direct inhibition of calcium-crystal formation.

RAS is an important player in cardiovascular disease, being involved in pathological processes in both the valve and myocardium in CAVS. ACE-inhibitors and ARBs are well-known attenuators of RAS effects. However, observational retrospective studies investigating the ACE-inhibitor effects on CAVS progression provided conflicting results. Treatment with ACE-inhibitors was associated with less aortic valve calcification ${ }^{26}$, but did not appear to slow hemodynamic progression. ${ }^{27}$ In principle ARBs might have superior effects on both valve fibrosis and calcification ${ }^{28}$, but prospective RCTs are lacking. With respect to the LV hypertrophic response, the RIAS RCT showed a modest but significant reduction of myocardial hypertrophy in patients with CAVS treated with ramipril. ${ }^{20}$ Finally, clinical observational studies have suggested that ACE-inhibitors and ARBs are associated with favourable effects on symptoms (dyspnea and exercise tolerance) and improved survival in patients with CAVS. ${ }^{30}$ Again RCT data is lacking.

\section{Statins}

Statins are widely used for lipid lowering in atherosclerosis and inflammation, being a specific inhibitor of hydroxymethylglutarylco-enzyme A-reductase (HMG-CoA-reductase). Whilst retrospective studies 
suggested that statins might also be of benefit in CAVS, subsequent RCTs demonstrated that statins in fact have no effect on CAVS progression or clinical outcomes. This conclusion was confirmed by a subsequent meta-analysis. ${ }^{31}$ The most plausible explanation for this failure is that whilst statins might intervene with inflammation and lipid deposition in the initiation phase, they have little effect once the propagation phase has become established when fibrosis and calcification are the dominant pathological processes.

\section{Lipoprotein(a) (Lp(a))}

Lp(a), the preferential plasma carrier of oxidized phospholipids (oxPL), is an LDL-like particle, containing additional apolipoprotein(a) and apolipoprotein-B100. A causal relationship between aortic valve calcification and a single-nucleotide polymorphism in the LPA-locus was suggested. ${ }^{32}$ Whilst the precise mechanisms of action of $L p(a)$ require further elucidation there is considerable interest in investigating whether $L p(a)$ is a modifiable target in CAVS. Statins are ineffective in reducing $\operatorname{Lp}(a)^{33}$, however several other therapeutic agents are currently in different stages of investigation. IONIS-APO(a) $)_{p \times}$ and IONIS-APO(a)- $\mathrm{L}_{\text {px }}$ ([Ligand-conjugated] antisense oligonucleotides targeting hepatic apolipoprotein(a) mRNA) have been investigated in phase 1 and 2 trials, demonstrating an ability to reduce $\operatorname{Lp}(a)$ concentrations. ${ }^{34}$ Other promising $\operatorname{Lp}(\mathrm{a})$ lowering alternatives are proprotein convertase subtilisin/kexin type 9 (PCSK9) inhibitors and Niacin. ${ }^{35}$ The effects of Niacin/PCSK9 on aortic stenosis are currently being investigated ("EAVaLL". Clinicaltrials. gov identifier: NCT02109614 and "PCSK9 inhibitors in the progression of aortic stenosis", Clinicaltrials.gov identifier: NCT03051360).

\section{Bisphosphonates and denosumal}

The calcification paradox implies that treatments for bone diseases (f.i. bisphosphonates or denosumab) might have a beneficial effect on vascular and valvular calcification whilst maintainin bone health. ${ }^{36}$ Bisphosphonates inhibit osteoclast-mediated bone resorption, resulting in decreased bone loss..$^{37}$ The inhibitory effect of bisphosphonates on vascular calcification was demonstrated in animals. ${ }^{38}$ Retrospectively, a delay in CAVS progression was confirmed ${ }^{39}$, whereas a more recent study failed to show a positive effect on hemodynamic CAVS progression or survival. ${ }^{40}$ These data are however confounded by the disease accelerating effects of osteoporosis. The on-going SALTIRE 2 (Clinicaltrials.gov identifier: NCT02132026) RCT will help determine the true impact of bisphosphonates. Denosumab, a human monoclonal antibody targeting RANKL, has been investigated in pre-clinical models. Its binding prevents the interaction between RANK and RANKL, resulting in inhibition of vascular calcification in mice. ${ }^{41}$ It too is being investigated as part of SALTIRE 2.

\section{Vitamin K}

Vitamin $\mathrm{K}$ is a fat-soluble vitamin consisting of two forms, namely phylloquinone (vitamin K1, VK1) present in green leafy vegetables and menaquinones (vitamin K2, VK2) present in fermented food. Long chain menaquinones (i.e. MK7) are transported more efficiently to extra-hepatic tissues. However, dietary intake of vitamin $\mathrm{K}$ is not sufficient to ensure full activation of MGP. ${ }^{19}$ Vitamin K supplementation is an attractive option to replenish vascular vitamin $\mathrm{K}$ stores to ensure optimal calcification inhibition. Vitamin K-supplementation in rats showed regression of warfarin-induced vascular calcification..$^{43}$ The prospective Rotterdam study was the first to report that dietary intake of VK2 showed an inverse relation with vascular calcification and mortality. ${ }^{44}$ Furthermore, low vitamin K status was shown to be associated with increased ucMGP levels and coronary artery calcification. ${ }^{19}$ Although promising, these studies were limited by the short-term follow-up, precluding measurable effects on clinical endpoints. Recently, the first in-man RCT demonstrated that vitamin K supplementation decelerated valvular calcification on CT in a small group of patients with CAVS. ${ }^{45}$ The effectiveness of vitamin $\mathrm{K}$ supplementation to reduce or hold calcification progression is currently subject of investigation in multiple trials ("iPACK-HD". Clinicaltrials.gov identifier: NCT01528800, "VitaVasK". ClinicalTrials. gov identifier: NCT01742273, "VitaK-CAC trial". ClinicalTrials.gov identifier: NCT01002157,“BASIK2”. ClinicalTrials.gov identifier: NCT02917525).

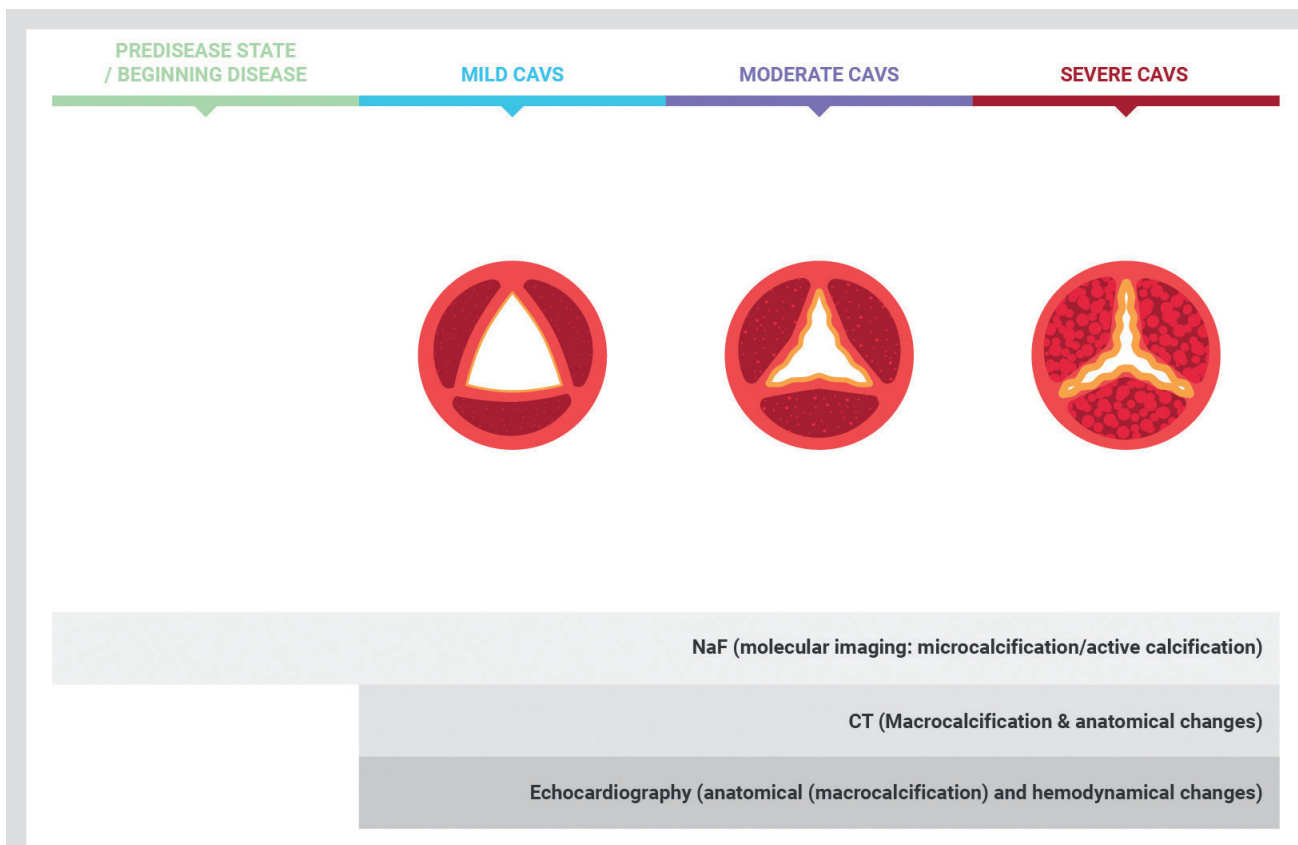

Figure 4. Role of imaging techniques in displaying calcification in stages of disease progression. Molecular ima ging using ${ }^{18} \mathrm{~F}-\mathrm{NaF}$ enables to visualize microcalcification and active calcification from beginni stages onwards. CT displays macrocalcification and anatomical changes in latter phases. Echocardiography visualizes anatomical changes and hemodynamic changes. ${ }^{2}$

\section{IMAGING IN CAVS: FROM ASSESSMENT OF HEMODYNAMICS TO DISEASE} PROGRESSION

The development of potential CAVS therapies creates a need for novel imaging techniques to assess their efficacy in phase 2 clinical trials. These would select the most promising agents to proceed to larger and more expensive phase 3 trials incorporating clinical endpoints. Several of such imaging approaches are discussed below and are listed in Figure 4.

\section{Echocardiography}

Echocardiography is the most commonly used imaging technique to assess patients with aortic stenosis, providing detailed information on aortic stenosis severity, LV wall thickness and function. 
Despite slow annual rate of CAVS progression and relatively high scan-rescan variability echocardiography is the most commonly used method for assessing aortic stenosis progression.

Another application of echocardiography is quantification of valve calcification, using a sem quantitative 4-point scale. Whilst echo-assessed calcification is an independent predictor of events (death or AVR) and disease progression ${ }^{46}$, it is not widely used largely because of poor reproducibility and repeatability.

\section{Computed Tomography (CT)}

Non-contrast multislice $\mathrm{CT}$ (MSCT) provides a more detailed and reproducible calcification scoring system. CT aortic valve calcium scoring (CT-AVC) enables quantification of mass, density and volume of macroscopic valvular calcification, expressed in Agatston units (AU), similar to the approach developed for the coronary arteries. CT-AVC correlates well with hemodynamic parameters on echocardiography. ${ }^{47}$ Interestingly, women require less calcification to develop severe CAVS than men, resulting in gender specific CT-AVC thresholds for severe CAVS (1275AU/2065AU for females/males), with additional prediction of subsequent disease progression and clinical events. ${ }^{48}$ Furthermore CT-AVC demonstrates relatively large annualized changes and specific calcification patterns provide additional insight for surgical and TAVI planning. ${ }^{49} \mathrm{CT}-\mathrm{AVC}$ is therefore appealing as an alternative method to assess disease severity and progression, and was recommended in the recent ESC guidelines for this purpose. ${ }^{2}$ Although CT-AVC provides excellent quantification of the established valve calcific burden it does not inform about disease activity or the biological mechanisms underlying CAVS.

\section{Positron Emission Tomography (PET)}

In contrast to echocardiography and CT, PET is an imaging technique that informs about the activity of specific biological processes. Inflammation and calcification can both be targeted using the PET tracers ${ }^{18} \mathrm{~F}$-fluorodeoxyglucose $\left({ }^{18} \mathrm{~F}\right.$-FDG) and ${ }^{18} \mathrm{~F}$-sodium fluoride $\left({ }^{18} \mathrm{~F}-\mathrm{NaF}\right)$, respectively. ${ }^{18}$ F-FDG has been applied to quantify vascular inflammation in the carotid arteries, correlating with macrophage infiltration..$^{50}$ Increased valvular ${ }^{18} \mathrm{~F}-\mathrm{FDG}$ uptake was demonstrated recently in CAVS and associated with faster subsequent disease progression. ${ }^{51-53}$ However, assessment of valvular ${ }^{18} \mathrm{~F}-\mathrm{FDG}$ activity is frequently obscured by uptake in the adjacent myocardium ${ }^{54}$ and may reflect glucose utilisation by a range of different cells or stimulating mechanisms. ${ }^{55}{ }^{18} \mathrm{~F}-\mathrm{NaF}$ has been used for many decades for the detection of bone metastases and primary osteoblastic tumors. ${ }^{56}$ In the vasculature it has been used to image developing microcalcification in carotid coronary and aortic atheroma ${ }^{57,58}$ and in CAVS, ${ }^{59}$ providing complementary information to CT-AVC. Indeed a striking mismatch has consistently been observed between the localization of the macroscopic calcium deposits on $\mathrm{CT}$ and the developing microcalcification identified by ${ }^{18} \mathrm{~F}-\mathrm{NaF}$. ${ }^{18} \mathrm{~F}-\mathrm{NaF}$ preferentially adsorbs to the available surface area of active hydroxyapatite crystal growth in areas of microcalcification whilst uptake is low in regions with established areas of macroscopic calcification. ${ }^{60}$ Histological validation of ${ }^{18} \mathrm{~F}-\mathrm{NaF}$ uptake in the valve in CAVS has been provided demonstrating a close correlation with proteins involved in active calcification. ${ }^{59}$

Prospective longitudinal studies have demonstrated that areas of microcalcification on ${ }^{18} \mathrm{~F}-\mathrm{NaF}$ PET develop with time into novel areas of macroscopic calcification. Thus ${ }^{18} \mathrm{~F}-\mathrm{NaF}$ PET acts as a good predictor of early disease progression in CAVS. ${ }^{52,59} \mathrm{On}$ this basis ${ }^{18} \mathrm{~F}-\mathrm{NaF}$ serves as a marker of calcification activity in CAVS and holds major potential as a surrogate end point to test the efficacy of novel pharmacological interventions.

\section{CONCLUSION AND FUTURE PERSPECTIVES}

AVS represents an increasing health care burden, leading to either adverse events or the requirement for major heart surgery. The pathophysiological mechanisms involved in CAVs initiation and progression are being rapidly elucidated and include inflammation, fibrosis and calcification. With this advancing knowledge we have identified novel therapeutic targets like vitamin $\mathrm{K}$ and new imaging techniques such as ${ }^{18} \mathrm{~F}-\mathrm{NaF}$ PET that can be used to test the efficacy of novel agents and further inform our pathophysiological understanding. Indeed several potential pharmacological treatments and under current investigation to achieve the ultimate goal: the inhibition of disease progression in CAVS. 


\section{REFERENCES}

Bonow RO, Greenland P. Population-wide trends in aortic 2. Baumgartner H. Falk V. Bax JJ et al. 2017 ESC/EACTS Guidelines for the management of valvular heart disease: The Task Force for the Management of Valvular Heart Disease of the European Society of Cardiology (ESC) and the Euro-
pean Association for Cardio-Thoracic Surgery (EACTS).

et al. Aortic stenosis, atherosclerosis, and skeletal bone: is there a common link with
calcification and inflammation? European heart journ 2013;34:1567-74.

4. Aikawa E, Libby P. A Rock and a Hard Place: Chiseling Away 2017:135:1951-1955.

5. Hutcheson JD, Aikawa E, Merryman WD. Potential drug targets for calcific aortic valve ology 2014;11:218-31.

6. Antonini-Canterin F, Leiballi E, Enache Ret al. Hydroxymethylglutaryl coenzyme-a reductase inhibitors delay the proechocardiographic study Journal of the American Coterm of Cardiology 2009:53:1874-9.

Roberts WC, Ko JM. Frequency by decades of unicuspid, bicuspid, and tricuspid aortic valves in adults having isowithout assoce replacement for aortic stenosis, with or without associated aortic regurgitation. Circulatio

inflammatory sta. Molecular imaging insights into earl tion. Circulation research 2011;108:1381-91.

Stewart BF, Siscovick D, Lind BK et al. Clinical factors associated with calcific aortic valve disease. Cardiovascul College of Cardio

Aikawa, E, Nahrend

moled activities in early aortic value disease. Circulation 2007;115:377-86

11. Otto CM, Kuusisto J, Reichenbach DD, Gown AM, O'Brie KD. Characterization of the early lesion of 'degenerative' chemical studies, Circulation 1994:90:844-53.

12. Sun L, Chandras, Sucosky P. Exvivo evidence for the contri-

bution of hemodynamic shear stress abnormalities to the early pathogenesis of calcific bicuspid aortic valve disease. Plos one 2012; 7: 48843

3. Hutcheson JD, Goettsch C, Bertazzo S et al. Genesis and in atherosclerotic plaques. Nature materials 2016:15:335-

14. Kim KM. Calcification of matrix vesicles in human aortic valve and aortic media. Federation proceeding 1976;35:156-62.

15. ElA Accaoui RN, Gould ST, Haji GP et al. Aortic valve sclerosis 2014;306: H1302-13.

16. Pawade TA, Newby DE, Dweck MR. Calcification in Aortic
Stenosis: The Skeleton Key Journal of the American College Sordiology 2015; 66:561-77.
of Card

17. Prakash SK, Bosse Y, Muehlschlegel JD et al. A roadmap to
(Bicuspid Aortic Valve Consortium). Journal of the Ameri(a)

Nigam V, Srivastava D. Notch1 represses osteogenic pathWers in aortic valve cells. Journal of molecular and cellular

Schurgers $L J$, Uitto J, Reutelingsperger CP. Vitamin K-dependent carboxylation of matrix Gla-protein: a crucia lar medicine 2013:19:217-26.

Luo G, Ducy P, Mckee MD et al. Spontaneous calcification of arteries and cartilage in mice lacking matrix GLA protein. Nature 1997;386:78-81.

Price PA, Faus SA, Williamson MK. Warfarin causes rapid valves. Arteriosclerosis, thrombosis, and vascurand hea 1998;18:1400-7.

Schurgers 1 , Aebert H, Vermeer C, Bultmann B, Janzen Oral anticoagulant treatment: friend or foe in cardiovascular disease? Blood 2004;104:3231-2.

Wijis B, Blaaun w, Rennenberg RJ et al. Patients using vitacation: an observational study in low-risk atrial fibrillation patients. European heart journal 2011;32:2555-62.

Gerdts E, Rossebo AB, Pedersen TR et al. Relation of Left Ventricular Mass to Prognosis in Initially Asymptomatic Mild to Moderate Aortic Valve Stenosis. Circulation Cardio-

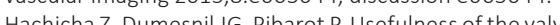

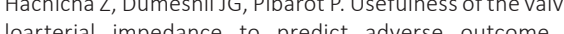
asymptomatic aortic stenosis, Journal of the American Colege of Cardiology 2009; 54:1003-11.

O'Brien KD, Probstfield JL, Caulfield MT et al. Angiotensin-converting enzyme inhibitors and change in aortic valve

Calcium. Archives of internal mediche 2005,165.858-62.

sin-converting enzyme inhibitors delay progression of aortic stenosis. Circulation 2004;110:1291-5.

Cote N, Mahmut A, Fournier D et al. Angiotensin receptor blockers are associated with reduced fibrosis and interleukin-6 expression in calcific aortic valve disease. Pathobioogy : journal of immunopathology, molecular and cellu

列 blind, randomized controlled trial of the angiotensin-converting enzyme inhibitor Ramipril In Aortic Stenosis (RIAS
trial). European heart journal cardiovascular Imagin 2015;16:834-41.

Nadir MA, Wei L, Elder DHJ et al. Impact of Renin-Angioten. 2011;58:570-576

Teo KL, Corsi DJ, Tam JW, Dumesnil JG, Chan KL. Lipid lowering on progression of mild to moderate aortic stenosis: meta-analysis of the randomized placebo-controlled clincaltials 2011:27:80 pat

作 New England journal of medicine 2013:368:503-12.

Thanassoulis G. Lipoprotein (a) in calcific aortic valve disease. from genomics to novel arug target for aotic stenosis. Joumalor lipid research 2016,57.917-24

nucleotides targeting apolipoprotein(a) in people with raised lipoprotein(a): two randomised, double-blind, placebo-conCrolled, dose-ranging trials. Lancet 2016,388.22 239-2253.

Much to tearn Jurnat of the Americon Hert Assocition 2016;5.

36. Persy V, D'Haese P. Vascular calcification and bone disease: 2009;15:405-16.

Sa

38. Price PA, Faus SA, Williamson MK. Bisphosphostes atendronate and ibandronate inhibit artery calcification doses comparable to those that inhibit bone resorption. Arteriosclerosis, thrombosis, and vascular biology 2001;21:817-24.

Innasimuthu AL, Katz WE. Effect of bisphosphonates on the progression of degenerative aortic stenosis. EchocardiogAksoy O Cam A

A 作

lege of Cardiology 2012;59:1452-9. tor activator of NF-kappaB ligand by denosumab atten ates vascular calcium deposition in mice. The America

pathways of K-vitamins in healthy subjects. Bion transport biophysica acta 2002;1570:27-32.

43. Schurgers LI, Spronk HM, Soute BA, Schiffers PM, DeMey 6 , Vermeer. Regression of warfarin-induced medial elastocalcinosis by high intake of vitamin $\mathrm{K}$ in rats. Bloo

reer C, Grobbee DE et al. Dietary intake of menaquinone is associated with a reduced risk of coronutrition 2004;134:3100-5.

45. Brandenburg VM, Reinartz $\mathrm{S}$, Kaesler $\mathrm{N}$ et al. Slower Prog ress of Aortic Valve Calcification With Vitamin K Supple mentation: Results From a Prospective Interventional
Proof-of-Concept Study. Circulation 2017:135:2081-2083.

6. Rosenhek R, Klaar U, Schemper M et al. Mild and modera aortic stenosis. Natural history and risk stratification by echocardiography. European heart journal 2004;25:199-205. . Cowell SJ, Newby DE, Burton J et al. Aortic valve calcifica tion on computed tomography predicts the severity of ao aortic valve calcification, as measured by $M D C T$, on surviva registry study. Journat to the American College of Cardioogy 2014;64:1202-13.

4. Sturla $F$, Ronzoni $M$, Vitali $M$ et al. Impact of different aortic valve calcification patterns on the outcome of transcathe nal of biomechanics 2016;49:2520-30.

50. Tawakol A, Migrino RQ, Bashian $\mathrm{GG}$ et al. In vivo 18F-fluorodeoxyglucose positron Bmission tomography maghng provides a noninvasive measure of carotid plaque inflammaCardiology 2006:48:1818-24.

51. Abdelbaky A, Corsini E, Figueroa AL et al, Early aortic valve inflammation precedes calcification: a longitudinal FDGPET/CT study. Atherosclerosis 2015;238:165-72.

52. Dweck MR, Jones C, Joshi NV et al. Assessment of valvular calification and intammation by positron emission 2012;125:76-86.
Jenkins WS, Vesey AT, Shah AS et al. Valvular (18)F-Fluoride and (18)F-Fluorodeoxyglucose Uptake Predict Disease Proanal of the American (1) 18F-sodian fluoride uptake. a novel marker of plaque bioof. Journal of the American College of Cardiology

Folco EJ, Sheikine Y, Rocha VZ et al. Hypoxia but not inflammation augments glucose uptake in human macrophages ing atherosclerosis with 18 fluorine Journal of the American College of Cardiology 2011;58:603

Grant FD, Fahey FH, Packard AB, Davis RT, Alavi A, Treves ST. Skeletal PET with 18F-fluoride: applying new technology to tion, Society of Nuclear Medicine 2008:49:68-78.

Derlin T, Richter U, Bannas $P$ et al. Feasibility of 18 F-sodium fluoride PET/CT for imaging of atherosclerotic plaque. Journal of nuclear medicine : official publication, Society Nerlin T, Wisotzki $C$, Richter $U$ et eral deposition in carotid plaque using 18 F-sodium fluorid nuclear medicine : official publication, Society of Nuclear Medicine 2011;52:362-8,

DweckMR, Jenkns Ws, Vesey AT et al. 18F-sodium fluorid uptake is a marker of active calcification and disease provascular imaging 2014; 7:371-8.

Irkle A, Vesey AT, Lewis DY et al. Identifying active vascular microcalcification by (18)F-sodium fluoride positron emission tomography. Nature communications 2015;6:749. 


\section{Vitamin K Antagonists, Non-vitamin K Antagonist Oral AntiCoagulants and vascular calcification in patients}

with atrial fibrillation

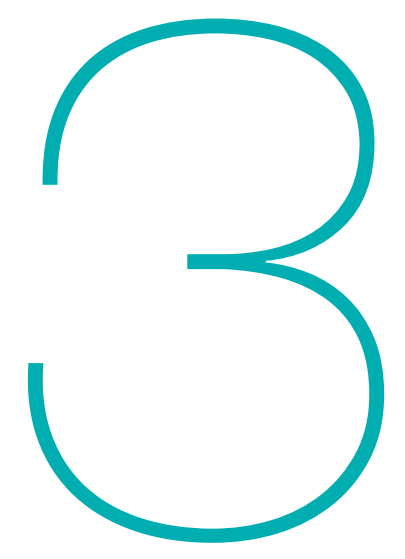

Frederique E.C.M. Peeters ${ }^{1}$, Elton A.M.P. Dudink ${ }^{1}$, Dorien M. Kimenai ${ }^{2}$, Bob Weijs ${ }^{1}$, Sibel Altintas ${ }^{1}$, Luuk I.B. Heckman ${ }^{1}$, Casper Mihl ${ }^{3}$, Leon J. Schurgers ${ }^{4}$, Joachim E. Wildberger', Steven J.R. Meex², Bas L.J.H. Kietselaer ${ }^{5}$, Harry J.G.M. Crijns

${ }^{1}$ Maastricht University Medical Center+ and CARIM, department of Cardiology, Maastricht, the Netherlands,

${ }^{2}$ Maastricht University Medical Center+, department of Clinical Chemistry, Maastricht, the Netherlands,

${ }^{3}$ Maastricht University Medical Center+ and CARIM, department of Radiology and Nuclear Medicine, Maastricht, the Netherlands,

${ }^{4}$ Maastricht University and CARIM, department of Biochemistry, Maastricht, the Netherlands,

${ }^{5}$ Maastricht University Medical Center+, department of Cardiology. Current affiliation: Zuyderland Medical Center, department of Cardiology, Heerlen/Sittard, the Netherlands 


\section{ABSTRACT}

Background: Vitamin K antagonists (VKA) are associated with coronary artery calcification in low-risk populations, but their effect on calcification of large arteries remains uncertain. The effect of non-vitamin $\mathrm{K}$ antagonist oral anticoagulants (NOAC) on vascular calcification is unknown. We investigated the influence of VKA and NOAC-use on calcification of the aorta and aortic valve.

Methods: In patients with atrial fibrillation without a history of major adverse cardiac or cerebrovascular events who underwent computed tomographic angiography, presence of ascending aorta (AsAC), descending aorta (DAC) and aortic valve (AVC) calcification was determined Confounders for VKA/NOAC treatment were identified and propensity score adjusted logistic regression explored the association between treatment and calcification (Agatston score $>0$ ). AsAC, DAC and AVC differences were assessed in propensity score matched groups.

Results: Of 236 patients (33\% female, age 58ะ9years), 71(30\%) used VKA (median duration: 122 weeks) and 79(34\%) used NOAC (median duration: 16 weeks). Propensity score adjusted logistic regression revealed that VKA-use was significantly associated with AsAC (OR2.31 (95\%Cl: 1.16-4.59), $p=0.017)$ and DAC (OR2.38 (95\%Cl: 1.22-4.67), $\mathrm{p}=0.012)$ and a trend in AVC (OR1.92 (95\%Cl0.983.80), $p=0.059)$ compared to non-anticoagulation. This association was absent in NOAC vs. non-anticoagulant (AsAC OR0.51 (95\%Cl: 0.21-1.21), p=0.127; DAC ORO.80 (95\%Cl: 0.36-1.76) $p=0.577$, AVC OR0.62 (95\%Cl:0.27-1.40), $p=0.248$ ). 178 patients were propensity score matched in three pairwise comparisons. Again, VKA-use was associated with DAC $(p=0.043)$ and a trend towards more AsAC ( $p=0.059)$, while NOAC-use was not (AsAC $p=0.264$; DAC $p=0.154$; AVC $p=0.280$ ).

Conclusions: This cross-sectional study shows that VKA-use seems to contribute to vascular calcification. The calcification effect was not observed in NOAC-users.

Key words: Cardiac computer tomographic (CT) imaging; aortic and arterial diseases; atrial fibrillation oral anticoagulant treatment 
via, Siemens Healthineers, Forchheim, Germany). For quantitative assessment of aortic valve and ascending and descending aorta calcification, the Agatston score was determined using a $3 \mathrm{~mm}$ CT slice thickness and a detection threshold of $\geq 130 \mathrm{HU}$ involving $\geq 1 \mathrm{~mm} 2$ area/lesion (3 pixels). ${ }^{1}$ Presence of calcification of the ascending aorta (AsAC), descending aorta (DAC) and aortic valve calcification (AVC) was defined as Agatston score $>0$. Calcium localized above the origin of the right coronary artery to the end of scan range, or up to the origin of the brachiocephalic artery was considered to be in the ascending aorta. Calcium present distal from the origin of the left subclavian artery up to the diaphragm was considered to be localized in the descending aorta. Two independent observers calculated the Agatston score of each segment separately, both blinded to medical data. In case of ambiguity, consensus was reached by discussion.

\section{Echocardiography}

An independent observer performed the echocardiography while subjects were lying in the left lateral decubitus position. Standard two-dimensional transthoracic echocardiography was performed, including M-mode, and Doppler echocardiography (Sonos 5500 and IE33, Philips Medical Systems, Andover, MA, USA) according to the guidelines of the European Association of Echocardiography. ${ }^{12}$

\section{Statistical analyses}

Statistical analyses were performed using SPSS version 22 (IBM Corp, Armonk, NY).

Normally distributed continuous variables are expressed as mean \pm standard deviation (SD) and compared using independent samples t-testing, non-normally distributed continuous variables median [interquartile range] (IQR) and compared using the Mann-Whitney $U$ test. Categorical variables are expressed as absolute numbers and percentages and tested using the $x^{2}$-test or Fishers exact test.

Patients were stratified according to type of treatment (non-anticoagulant, VKA or NOAC) and the effect on the primary outcome, the presence of calcification in the ascending and descending aorta and the aortic valve between the groups was investigated using propensity scores (Supplemental figure 1). Since patients were not randomly assigned to type of treatment, we adjusted for factors favouring non-anticoagulant, VKA or NOAC prescription using propensity score to reduce the selection bias and see the genuine effect of therapy on the primary outcome. Groups were compared in a pairwise manner ${ }^{13}$, and for every pair, two analyses were performed as previously described. Firstly, variables associated with VKA or NOAC treatment were identified by univariable logistic regression (retention level set at 0.1). Subsequently, propensity scores of the variables showing a significant relation with prescription of VKA or NOAC were calculated for each patient and integrated in the logistic regression models as a covariate with presence of calcification in the ascending aorta, descending aorta and the aortic valve as outcome variables. Secondly, a propensity score matched analysis was performed for every pair, in which patients were propensity score matched to patients in the treatment group they were to be compared with, using the Greedy matching strategy. ${ }^{13,14}$ Propensity scores were matched to the closes propensity score of a patient in the other treatment arm with a maximum difference of 5\%. a patil all patients were matched, or until the propensity scores differed $>5 \%$ between arms. The $\chi^{2}$-test was performed to assess the differences in presence of AsAC, DAC and AVC
The Jonckheere-Terpstra test was performed to assess whether duration of VKA treatment (in tertiles) was associated with the height of the total Agatston score in the VKA treatment group. A similar analysis could not be performed in NOAC-users, due to the shorter duration of treatment in this group. Overall, statistical significance was assumed for $\mathrm{P}<0.05$.

\section{RESULTS}

\section{Baseline variables}

Mean age $( \pm S D)$ of the total population was $58 \pm 9$ years, $67 \%(n=159)$ were male and the median [IOR] CHA DS -VASC score was 1.0 [1.0]. Indications for MSCT were work-up for ablation (51.5\%) and general check-up for coronary artery disease. Further baseline characteristics are shown in table 1. AsAC, DAC or AVC was present in 81 (34.3\%), 109 (46.2\%) and 88 (37.3\%) patients, respectively (Figure $1 \mathrm{a}$ and $1 \mathrm{~b}$ and Supplemental figure 2). A total of 47 (19.9\%) patients showed calcification of both the aortic segments and the aortic valve. Of the total study population, 86 patients (36.4\%) were in the non-anticoagulant group, 71 (30.1\%) in the VKA-group and $79(33.5 \%)$ in the NOAC-group. Median duration of VKA or NOAC treatment was 122 [IQR 51-209] and 16 [IQR 6-42] weeks, respectively.

\section{Table 1. Baseline characteristics of the total population}

\begin{tabular}{|c|c|c|c|c|}
\hline & $\begin{array}{l}\text { Total population } \\
(\mathrm{n}=236)\end{array}$ & $\begin{array}{l}\text { Non- anticoagulant } \\
(\mathrm{n}=86)\end{array}$ & $\begin{array}{l}\text { VKA } \\
(n=71)\end{array}$ & $\begin{array}{l}\text { NOAC } \\
(n=79)\end{array}$ \\
\hline \multicolumn{5}{|l|}{ Demographics } \\
\hline Age (years) & $58.3 \pm 9.2$ & $55.8 \pm 9.1$ & $57.9 \pm 9.0$ & $61.5 \pm 8.5$ \\
\hline Male sex & $159(67.4)$ & $53(61.6)$ & $57(80.3)$ & $49(62.0)$ \\
\hline $\mathrm{BMI}\left(\mathrm{kg} / \mathrm{m}^{2}\right)$ & $27.1 \pm 3.6$ & $26.6 \pm 3.3$ & $27.5 \pm 3.2$ & $27.2 \pm 4.2$ \\
\hline Smoking & $27(11.4)$ & $11(12.8)$ & $8(11.3)$ & $8(10.1)$ \\
\hline Positive family history (AMI) & $33(14.0)$ & $9(10.5)$ & $7(9.9)$ & $17(21.5)$ \\
\hline Systolic blood pressure $(\mathrm{mmHg})$ & $128.2 \pm 12.9$ & $125.5 \pm 9.9$ & $124.5 \pm 10.7$ & $134.4 \pm 15.1$ \\
\hline Fasting blood glucose (mmol/L) & $5.44 \pm 0.52$ & $5.43 \pm 0.43$ & $5.31 \pm 0.54$ & $5.58 \pm 0.56$ \\
\hline Cholesterol (mmol/L) & $5.40 \pm 0.96$ & $5.44 \pm 0.90$ & $5.47 \pm 1.14$ & $5.28 \pm 0.83$ \\
\hline Triglycerides (mmol/L) & $1.68 \pm 0.92$ & $1.56 \pm 0.82$ & $1.79 \pm 1.13$ & $1.70 \pm 0.78$ \\
\hline LDL (mmol/L) & $3.41 \pm 0.83$ & $3.55 \pm 0.73$ & $3.52 \pm 0.99$ & $3.16 \pm 0.74$ \\
\hline $\mathrm{HDL}(\mathrm{mmol} / \mathrm{L})$ & $1.27 \pm 0.35$ & $1.20 \pm 0.28$ & $1.21 \pm 0.41$ & $1.41 \pm 0.34$ \\
\hline Creatinine $(\mu \mathrm{mol} / \mathrm{L})$ & $86.7 \pm 13.9$ & $86.2 \pm 14.2$ & $88.9 \pm 12.6$ & $85.6 \pm 14.5$ \\
\hline PROCAM risk score (\%) & $7.0[7.0]$ & $5.7[7.6]$ & $7.0[9.3]$ & $7.0[6.0]$ \\
\hline $\mathrm{CHA}_{2} \mathrm{DS}_{2}$-VASc score & $1.0[1.0]$ & $1.0[1.0]$ & $1.0[1.0]$ & $1.0[1.0]$ \\
\hline $\begin{array}{l}\text { Anticoagulation duration } \\
\text { (weeks) }\end{array}$ & NA & NA & $122[158]$ & $16[36]$ \\
\hline AF duration (months) & 25 [70] & 26 [72] & 42 [71] & $8[24]$ \\
\hline
\end{tabular}




\begin{tabular}{|c|c|c|c|c|}
\hline & $\begin{array}{l}\text { Total population } \\
(\mathrm{n}=236)\end{array}$ & $\begin{array}{l}\text { Non- anticoagulant } \\
(n=86)\end{array}$ & $\begin{array}{l}\text { VKA } \\
\text { (n=71) }\end{array}$ & $\begin{array}{l}\text { NOAC } \\
(n=79)\end{array}$ \\
\hline \multicolumn{5}{|l|}{ Medication } \\
\hline Rhythm control & $146(61.9)$ & $51(59.3)$ & $51(71.8)$ & $44(55.7)$ \\
\hline Rate control & $120(50.8)$ & $41(47.7)$ & $40(56.3)$ & $39(49.4)$ \\
\hline ACE-inhibitors & $32(13.6)$ & $9(10.5)$ & $11(15.5)$ & $12(15.2)$ \\
\hline Angiotensin receptor blockers & $54(22.9)$ & $16(18.6)$ & $18(25.4)$ & $20(25.3)$ \\
\hline Diuretics & $33(14.0)$ & $8(9.3)$ & $12(16.9)$ & $13(16.5)$ \\
\hline Statins & $37(15.7)$ & $13(15.1)$ & $13(18.3)$ & $11(13.9)$ \\
\hline \multicolumn{5}{|l|}{ Echocardiography } \\
\hline LA dimension $(\mathrm{mm})$ & $41.1 \pm 5.2$ & $40.0 \pm 5.1$ & $42.5 \pm 5.0$ & $41.1 \pm 5.2$ \\
\hline LA volume (ml) & $76.8 \pm 23.6$ & $69.5 \pm 21.9$ & $80.3 \pm 21.6$ & $81.9 \pm 25.7$ \\
\hline RA volume (ml) & $60.8 \pm 24.8$ & $57.0 \pm 18.5$ & $62.2 \pm 22.7$ & $64.0 \pm 32.5$ \\
\hline IVS (mm) & $8.6 \pm 0.8$ & $8.5 \pm 0.8$ & $8.7 \pm 0.8$ & $8.7 \pm 0.8$ \\
\hline PW (mm) & $8.5 \pm 0.8$ & $8.5 \pm 0.7$ & $8.6 \pm 0.8$ & $8.5 \pm 0.8$ \\
\hline LVEF (\%) & $60.2 \pm 6.0$ & $60.9 \pm 6.1$ & $60.5 \pm 6.5$ & $59.1 \pm 5.1$ \\
\hline
\end{tabular}

Continuous variables are expressed as mean $\pm S D$ or median [IQR] depending on their distribution. Categorical variables are reported as $n(\%)$.

Abbreviations: AMI; acute myocardial infarction, $L A$; left atrium, RA; right atrium, IVS; interventricular septum, PW; posterior wall, LVEF; left ventricular ejection fraction

\section{Identification of confounders for VKA or DOAC treatmen}

Patients on VKA treatment were more likely to be male and treated with rhythm control medication than non-anticoagulant patients. Moreover, they were more likely to have a higher BMI and larger left atrial diameters. Compared to the non-anticoagulant or VKA group, NOAC users were older, more likely to have higher systolic blood pressure and their medical history with AF and thus anticoagulant treatment duration was shorter, however they had a comparable left atrial volume to the VKA-group. Additionally, they had a more favourable LDL and HDL cholesterol profile. In pairwise comparison between NOAC and VKA users, NOAC users were more likely to be of female sex and had slightly higher plasma glucose levels (Supplemental Table 1).

\section{Calcification differences between types of anticoagulation}

Distribution of prevalence of calcification of all segments of the three groups is displayed in Figure 1.

Regression analyses, adjusted for the propensity scores to correct for the identified confounders, revealed that patients in the VKA treatment group had significantly more AsAC and DAC when compared to the non-anticoagulation group (AsAC: OR 2.31, 95\% Cl 1.16-4.59, $p=0.017$; DAC: OR $2.38,95 \% \mathrm{Cl} 1.22-4.67, \mathrm{p}=0.012$ ) and showed a trend towards a higher calcium score of the aortic valve (AVC: OR 1.92, 95\% Cl 0.98-3.80, p=0.059) (Figure 2, panel A). AsAC, DAC and AVC were not significantly different between the non-anticoagulation and NOAC groups (AsAC: OR 0.51, 95\% C 0.21-1.21, p=0.127; DAC: OR 0.80, 95\% Cl 0.36-1.76, p=0.577; AVC: OR 0.62, 95\% Cl 0.27-1.40,

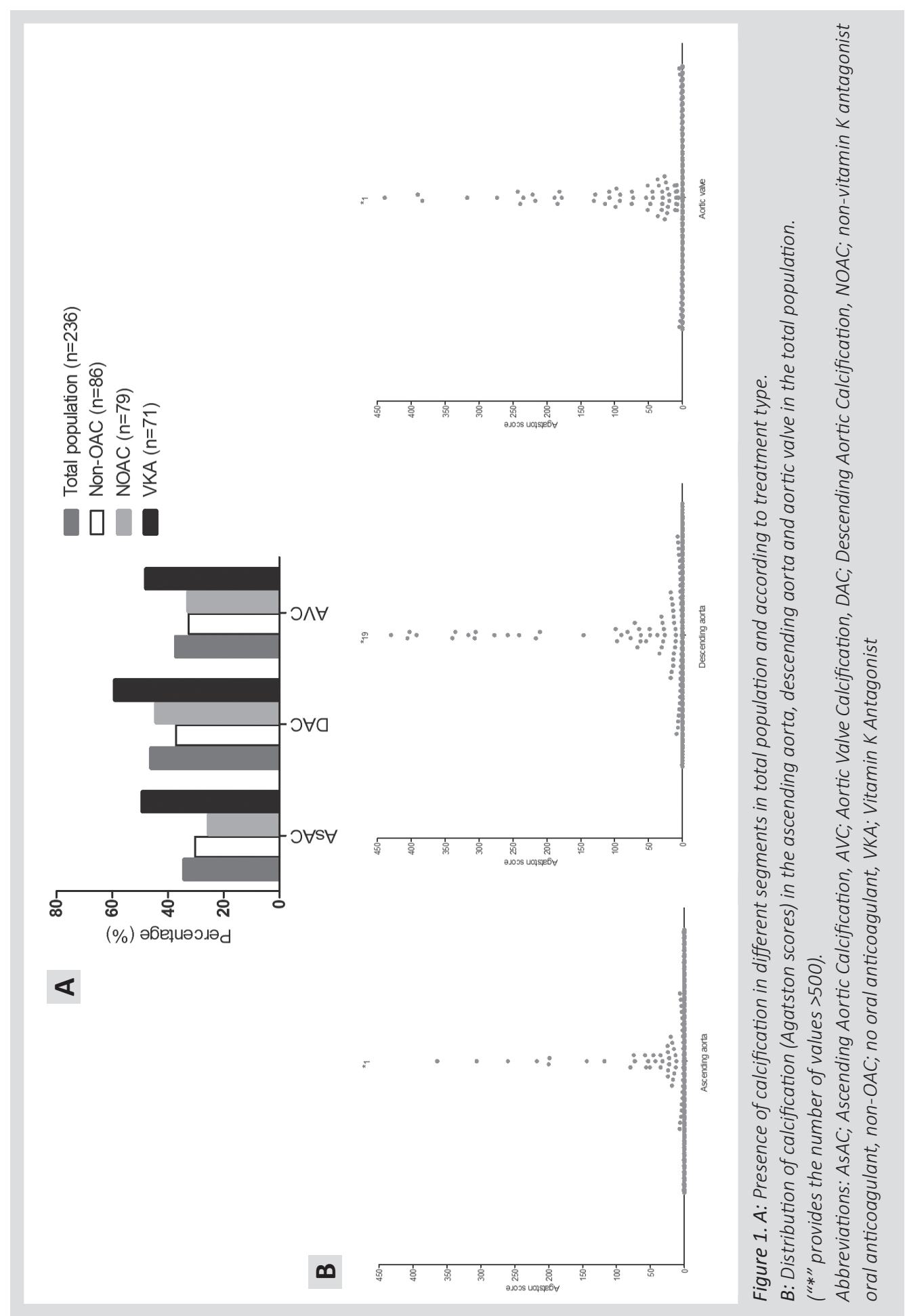




$2.31(1.16-4.59)$
$2.38(1.22-4.67)$
$1.92(0.98-3.80)$

Figure 2. AsAC, DAC and AVC in propensity score adjusted regression analyses (odds ratio $(95 \% \mathrm{CI})$ ). Panel A: non-anticoagulation (reference) vs. VKA. Panel B: non-anticoagulation (reference) vs. NOAC. Panel C: NOAC (reference) Vs. VKA.

Abbreviations: AsAC; Ascending Aortic Calcification, AVC; Aortic Valve Calcification, DAC; Descending Aortic Calcification, NOAC; non-vitamin K antagonist oral anticoagulant, VKA; Vitamin K antagonist

$p=0.248$ respectively) (Figure 2, panel B). Occurrence of DAC and AVC was significantly higher in the VKA group when compared to the NOAC group (DAC: OR 3.60, 95\% Cl 1.18-10.97, p=0.025; AVC: OR $3.26,95 \% \mathrm{Cl} 1.09-9.70, p=0.034)$. AsAC was higher in the VKA treatment group as compared with the NOAC group, although a significant difference was not reached (AsAC OR 2.39, 95\% Cl 0.82-6.92, $\mathrm{p}=0.109$ ) (Figure 2, panel C)

\section{Calcification differences in the propensity score matched population}

A total of 178 patients were propensity-score matched in three pairwise comparisons according to treatment type. For comparison between non-anticoagulation and the VKA group, 118 patients were matched (59 in each group). These numbers were 32 and 28 (16 and 14 per group) for the nonanticoagulation/NOAC and the NOAC/VKA comparisons, respectively. After the matching procedure, clinical conditions and echocardiographic measurements were identical (Supplemental table 2).

When comparing the presence of calcification between the propensity-score matched groups of non-anticoagulation and VKA, presence of DAC remained significantly higher in the VKA-treated group $(n=23(39.0 \%)$ vs. $n=34(57.6 \%), p=0.043)$, while AsAC showed a trend towards a higher presence $(n=18(30.5 \%)$ vs. $n=28(47.5 \%), p=0.059)$. AVC was not significantly different between the two groups ( $n=24(40.7 \%)$ vs. $n=29(49.2 \%) p=0.355)$ (Figure 3A).

The non-anticoagulation/NOAC matched cohort did not reveal significant differences of AsAC, DAC and AVC ( $n=7$ (43.8\%) vs. $n=4$ (25.0\%), $p=0.264 ; n=9$ (56.3\%) vs. $n=5$ (31.3), $p=0.154 ; n=8$

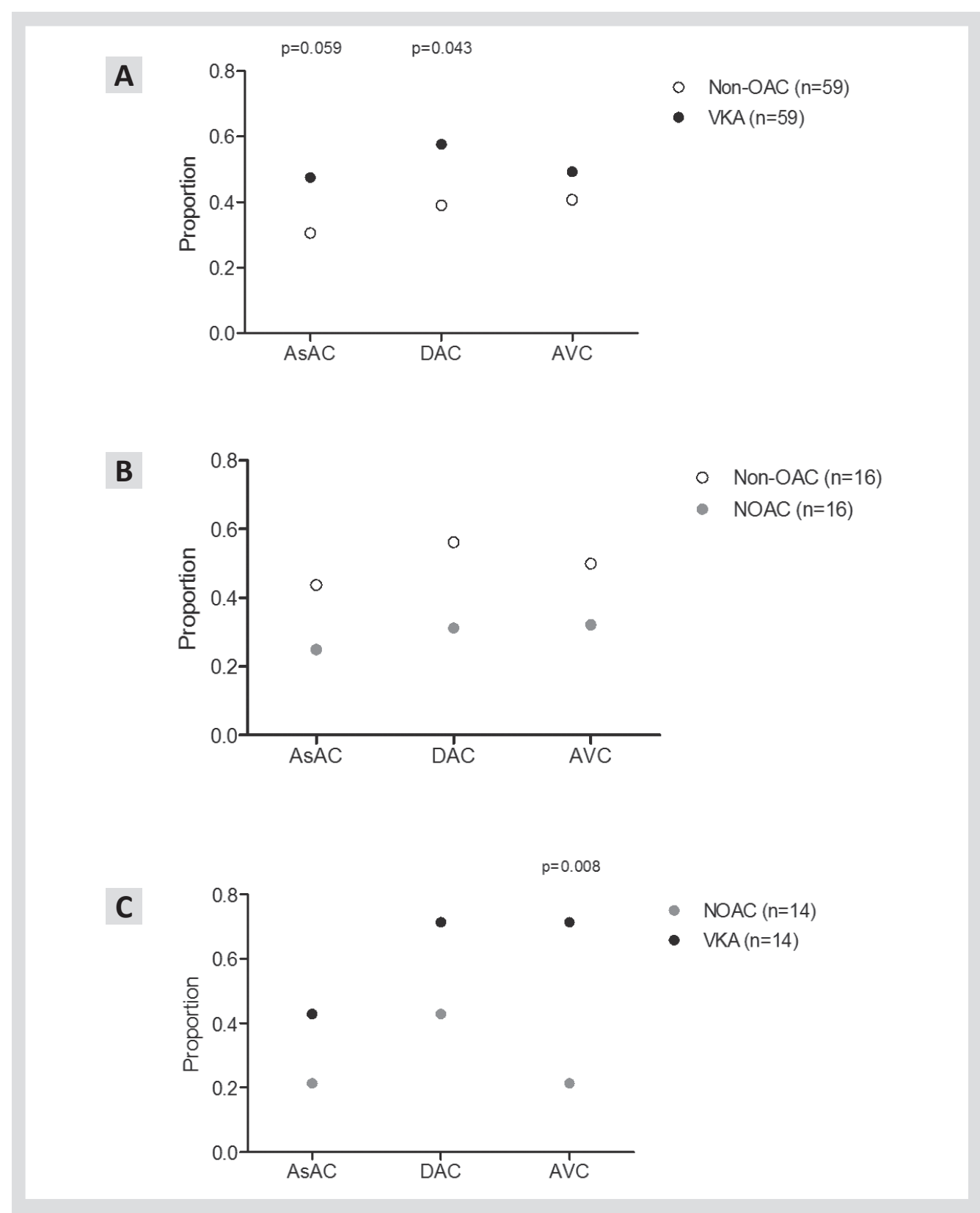

Figure 3. ASAC, DAC and AVC per treatment group after propensity score matching.

A: propensity score matched cohort non-anticoagulation/VKA. B: propensity score matched cohort non-anticoagulation/NOAC. C: propensity score matched cohort NOAC/VKA. Statistical testing using chi-square. Abbreviations: AsAC; Ascending Aortic Calcification, AVC; Aortic Valve Calcification, DAC Descending Aortic Calcification, NOAC: non-vitamin K antagonist oral anticoagulant, non-OAC non-anticoagulant, VKA: Vitamin K antagonist 
(50.0\%) vs. $n=5(31.3 \%), p=0.280$, Figure $3 B$ ). Less calcification in AsAC and DAC was seen in the NOAC group, although statistical significance was not reached $(p=0.225$ and $p=0.127)$, yet AVC was statistically significantly different between these groups ( $n=3$ (21.4\%) vs. $n=10(71.4 \%), p=0.008$, Figure 3C)

\section{VKA duration and calcification}

Duration of treatment with VKA differed between patients. The Jonckheere-Terpstra test revealed a statistically significant trend between the extent of total vascular calcification and increased duration of VKA treatment $(p=0.034)$. The rise in calcification levels and VKA duration is illustrated in Figure 4.

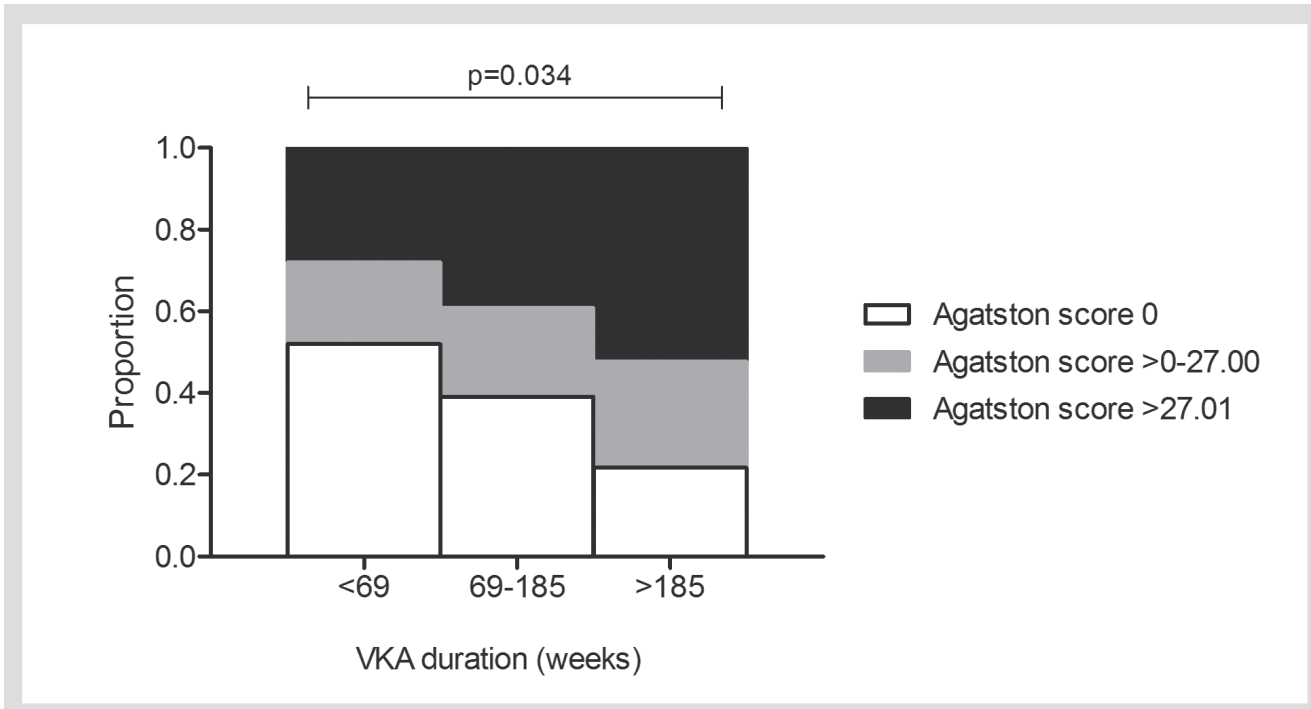

Figure 4. Vascular calcium score categories (tertiles) in patients with different treatment duration of vitamin Kantagonist (VKA). Statistical testing using Jonkheere-Terpstra test

\section{DISCUSSION}

This study investigated the effect of anticoagulant treatment with VKAs and NOACs on vascular calcification as compared to patients with AF not treated with oral anticoagulation. The main finding of this study is that treatment with VKA is associated with a higher prevalence of calcification of the thoracic aorta when compared to patients without oral anticoagulation or NOAC treatment, whereas a difference in calcification between the NOAC and group without oral anticoagulation was not observed.

\section{Vitamin $\mathrm{K}$ antagonists and vascular calcification}

Several studies describing populations with variable cardiovascular disease profiles reported an association between vascular and valvular calcification and VKA use. ${ }^{2-4}$ Additionally, two studies considering relatively low-risk populations reported an association between femoral artery calcification and coronary artery calcification and VKA treatment. ${ }^{9,15}$ Using propensity matched analyses, we provide a funded direction to causality and show that in patients with similar risk profiles, those treated with VKA have more calcification of systemic arteries and the aortic valve in comparison to patients not treated with VKA. With that, our results are in accordance with previous data and extend knowledge, derived from these studies involving patients with different risk profiles, all treated with VKA. Although former studies showed controversial results, 2,16 we observed a trend in the duration of VKA treatment towards increased vascular calcification in the present population, hinting towards a direct calcification-inducing effect of VKA.

\section{The mechanism connecting VKA and calcification}

VKAs are established in prevention of thrombo-embolic complications and exert their anticoagulant effect by inhibition of the activation of vitamin $\mathrm{K}$ dependent coagulation factors II, VII, IX and X. Bleeding is a major adverse effect of VKA and has been extensively described in literature However, the calcification-induction potential of VKAs is not considered in its prescription in daily clinical practice. This effect on calcification is caused by the inhibition of matrix- $\gamma$-carboxyglutamic acid (Gla) protein (MGP), a vitamin K-dependent protein synthesized by vascular smooth muscle cells (VSMC). ${ }^{17}$ The function of MGP as an inhibitor of soft tissue calcification was shown in MGP-deficient mice which developed extensive arterial calcification and died within 2 months due to aortic rupture. ${ }^{18}$ The effect of VKA on calcification was demonstrated in experimental animal models which showed similar development of calcification in vascular and aortic valve tissue as in MGP knock-out animals. ${ }^{19}$ The inhibition of MGP by VKA results in decreased calcium binding and inhibition of calcium crystals formation and bone morphogenetic protein (BMP) action ${ }^{18,20,21}$ The loss of inhibitory function is furthermore caused by a relative shortage of vitamin $\mathrm{K}$ due to upregulation of MGP in areas with calcification, resulting in a high ratio of uncarboxylated versus carboxylated MGP. The last phenomenon is clinically associated with an increased risk for arterial and coronary calcification. $22-24$

\section{VKA versus NOAC for patients with atrial fibrillation}

Aortic calcification is considered a subclinical marker of atherosclerosis, like coronary artery calcification, sharing risk factors with the latter. ${ }^{25}$ The prognostic value of cardiovascular calcification has clearly been demonstrated in several patient populations with cardiovascular disease (CVD), showing an increased risk of future events with increasing calcification. ${ }^{26,27}$ The negative pharmacological characteristics and adverse events associated with VKA-use opened the market for a new generation of anticoagulants: NOACs. ${ }^{1}$ NOACs exert their anticoagulant effect by direct inhibition of factors Ila or Xa, and thus NOACs do not interfere with vitamin K-dependen proteins such as MGP. ${ }^{17}$ In our study we found no difference between the group of patients treated with NOACs as compared to those without oral anticoagulation. Furthermore, although treatment duration has to be taken into account, we found less calcification in the NOAC group in comparison to the VKA group. Thereby, although preliminary, we provide evidence that NOACs lack the calcification-induction effect of VKAs. The effect of both VKA and NOACs on both valvular and vascular calcification (including coronary artery calcification) are currently investigated in randomized controlled trials considering populations with different cardiovascular disease profiles. $^{28}$

Next to the suggested absence of this calcification inducing effect, direct beneficial effects of NOACs on attenuation of atherosclerosis and plaque stability have been suggested in animal models, ${ }^{29}$ as NOACs inhibit PAR-receptor function through inhibition of factors Ila or Xa. Exemplar for this is that the COMPASS trial, investigating whether Rivaroxaban alone or added to aspirin leaded to less cardiovascular events in comparison to aspirin alone, was halted after the interim 
analysis revealed superiority of the combined Rivaroxaban plus aspirin in comparison to aspirin alone. ${ }^{30} \mathrm{As}$ AF is generally associated with an increased cardiovascular risk profile, this effect of NOACs can be expected to be of particular value in AF patients.

Overall, growing insight in the presence/absence of calcification-induction of different ora anticoagulants and their effect on atherosclerosis holds potential to provide important implications to aid clinicians in their choice for anticoagulant treatment type, especially in patients with lifelong indication for $\mathrm{OAC}$, such as patients with $\mathrm{AF}$.

\section{Strengths and limitations}

Our study creates the opportunity to assess the true effect of the anticoagulation type in patients with a comparable cardiovascular profile by excluding a number of potential variables that could have influenced this effect, providing a funded direction towards a causal relationship. We believe that our results are of additive value due to the method of analyses using propensity score matching. Although the current study provides important insight, some caution must be considered with the interpretation. First, the sample size of the study resulted in relatively small propensity matched arms of non-anticoagulation/NOAC and NOAC/VKA. Second, we tried to circumvent limitations generally involved in studies with a retrospective nature and a cross-sectional design. However, the design of the current study does not allow correcting for unmeasured confounders, whereby residual confounding could persist. Furthermore, levels of calcification before initiation of anticoagulation are unknown, and may have differed between patients. Also, the shorter duration of NOAC treatment in the current study has to be taken into account. Therefore, this result has a rather basic nature regarding the true (absent) effect of NOACs on calcification. Extrapolation of these results to a broader patient population should be done with caution. Studies on long term NOAC-use are necessary to confirm these findings and to provide valuable additional information in the determination of a true "absent" effect of NOACs on calcification.

\section{CONCLUSION}

Vascular calcification is known to be a marker of increased cardiovascular morbidity and mortality, making it non-negligible, certainly in initially low-risk populations. All studies performed so far suggest, but could not define true causality between VKA and aortic and valvular calcification due to their design. Yet in this study, we show that VKA contributes to the presence of vascular calcification, which is not observed in NOAC. To address the question whether VKAs cause vascular calcification and NOACs do not, randomized controlled trials are on their way.

\section{REFERENCES}

van Gorp RH, Schurgers LJ. New Insights into the Pros an 2015;7:9538-57.

2. Koos R, Mahnken AH, Muhlenbruch G et al. Relation of ora anticoagulation to cardiac valvular and coronary calcium assessed by multislice spiral computed tomography. Am mostasis : JTH 2009; 7:2023-7.

4. Schurgers LJ, Aebert H, Vermeer C, Bultmann B, Janzen Oral anticoagulant treatment: friend or foe in cardiovasc lar disease? Blood 2004,104.3231-2.

, hurgers L, van Engelshove marker of increased cardiovascular risk: a meta-ans alysis. Vasc Health Risk Manag 2009;5:185-97.

6. Kirchhof P, Benussi S, Kotecha D et al. 2016 ESC Guidelines for the management of atrial fibrillation developed in co2016:37.2893-2962.

Sterne JA, Bodalia PN, Bryden PA et al. Oral anticoagulants tion of venous thromboembolic disease, and for prevention of stroke in atrial fibrillation: systematic review, nework meta-analysis and cost-effectiveness analysis. Healt

. Morishima Y, Kamisato C, Honda Y, Furugohri T, Shibano $T$. an, an oral direct factor undercarboxylated osteocalcin (uc-osteocalcin) in rats. Thrombosis research 2013;131:59-63.

Weijs B, Blaauw $Y$, Rennenberg RJ et al. Patients using vitaCation: an observational study in low- of corrial fibrillation patients. European heart journal 2011:32:2555-62.

10. Assmann G, Cullen P, Schulte H. Simple scoring scheme for calculating the risk of acute coronary events based on the 10-year follow-up of the prospective cardiovascular Munster (PROCAM) study. Cliculation 2002,105:310-5.

WR, Hildner FJ, Zusmer NR, Viacalcium using ultrafast computed tomography. $\downarrow$. Am Col Cardiol 1990;15:827-32.

12. Evangelista A, Flachskampf F, Lancellotti P et al. European Association of Echocardiography recommendations for standardization of performance, digital storage and reportechocardiography : the journal of the Working Group on Echocardiography of the European Society of Cardiology 29. 2008;9:438-48.

13. Rassen JA, Shelat AA, Franklin JM, Glynn RJ, Solomon DH, Schneeweiss $S$. Matching by propensity score in coho studies with three treatment

14. Blackstone EH. Comparing ap

15. Rennenberg RJMW, van Varik BJ, Schurgers LJ et al. Chronic coumarin treatment is associated with increased extracoronary arterial calcification 5123 Holden RM, Sanfilippo AS, Hopman WM, Zimmerman D
Garland JS, Morton AR. Warfarin and aortic valve calcifica- tion in hemodialysis patients. Journal of nephrology 2007:20:417-22. K-dependent carboxylation of matrix Gla-protein: a crucia swe the to control ectopic mineralization. Trends Mol Med 2013;19:217-26.

of al. Spontaneous calcification

calcification of the elastic lamellae in rat arteries and heart valves. Arterioscler Thromb Vasc Biol 1998;18:1400-7.

. Dowd P, Hershline R, Ham SW, Naganathan S. Vitamin Kand energy transduction. a base strength ampl

tional modifications regulate matrix Gla protein funstioimportance for inhibition of vascular smooth muscle cell calcification. Journal of thrombosis and haemostasis : JTH 2007,5.2503-11.

Atsma $\mathrm{F}$ et al. High dietary menaqution. Atherosclerosis 2009:203:489-93. intake reduces the incidence of coronary heart disease. Nutr Metab Cardiovasc Dis 2009;19:504-10

. Characterisation (MGP) species. Thrombosis and hae Gla pro2010;104:811-22.

Kalsch H, Lehmmann N, Mohlenkamp $S$ et al, Prevalence of thoracic aortic calcification and its relationship to cardiovascular risk factors and coronary calcification in an unselected population-based cohort: the Heinz Nixdorf Recal 2013:29:207-16.

$\mathrm{N}$, Berg MH et al. Coronary artery calcfication outperforms thoracic aortic calcification for the prediction of myocardial infarction and all-cause mortality. the Heinz Nixdorf Recall Study. European journal of preventive cardiology 2014;21:1163-70.

政 of Coronary Atherosclerosis. Journal of the American Heart Association 2017;6.

. Caluwe R, Pyfferoen L, De Boeck K, De Vriese AS. The effects of vitamin $K$ supplementation and vitamin $K$ antagonists on progression of vascular calcification: ongoing ran2016;9:273-9

Kadoglou N. Moustardas P, Katsimpoulas M et al The beneficial effects of a direct thrombin inhibitor, dabigatran etexilate, on the development and stability of atheroscleroticlesions in apolipoprotein E-deficient mice: dabigatran etexilate and atherosclerosis. Cardiovascular drugs and

Eikelboom JW, Connolly SJ, Bosch J et al. Rivaroxaban wit New England journal of medicine 201 


\section{SUPPLEMENTAL MATERIAL}

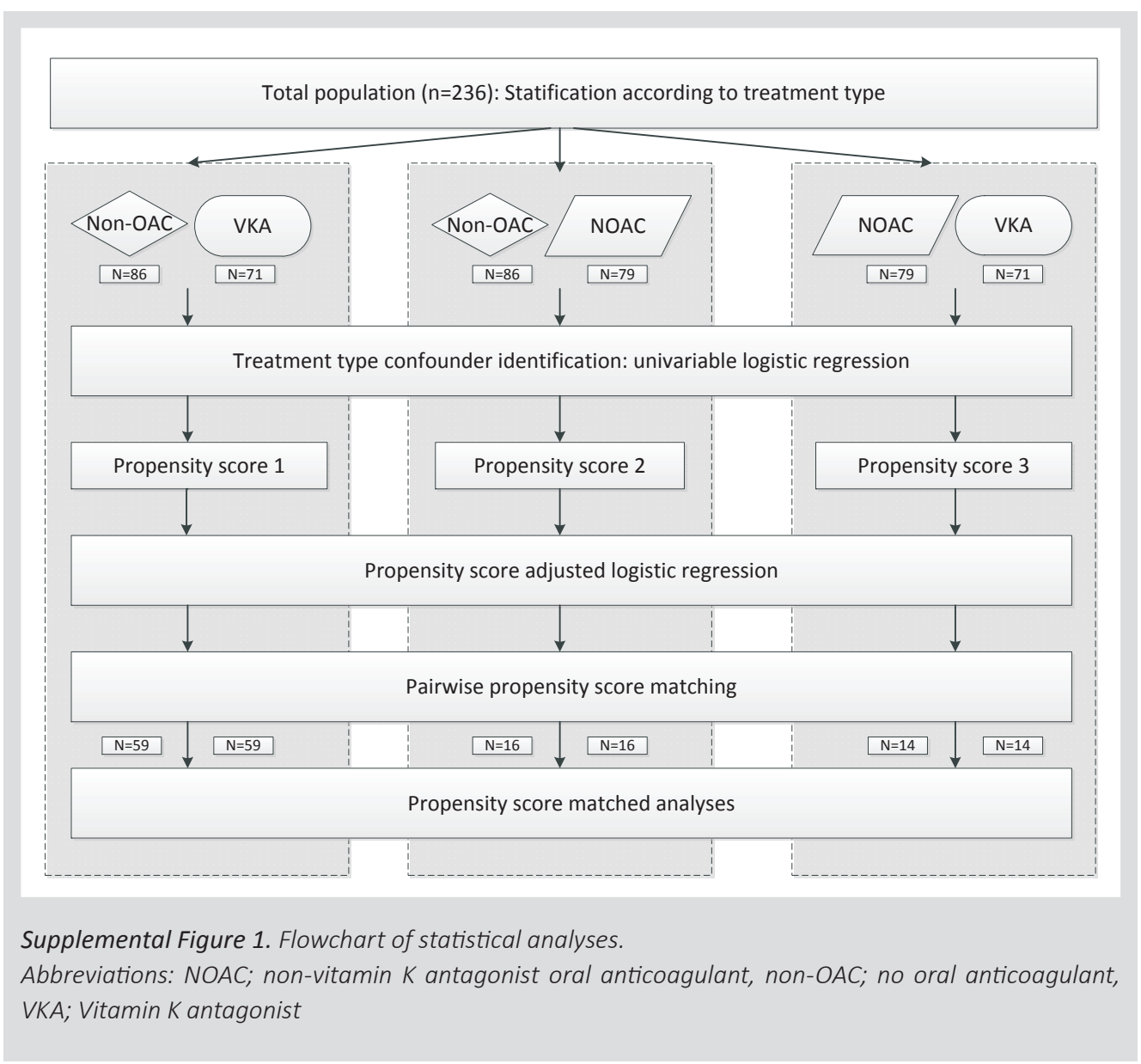




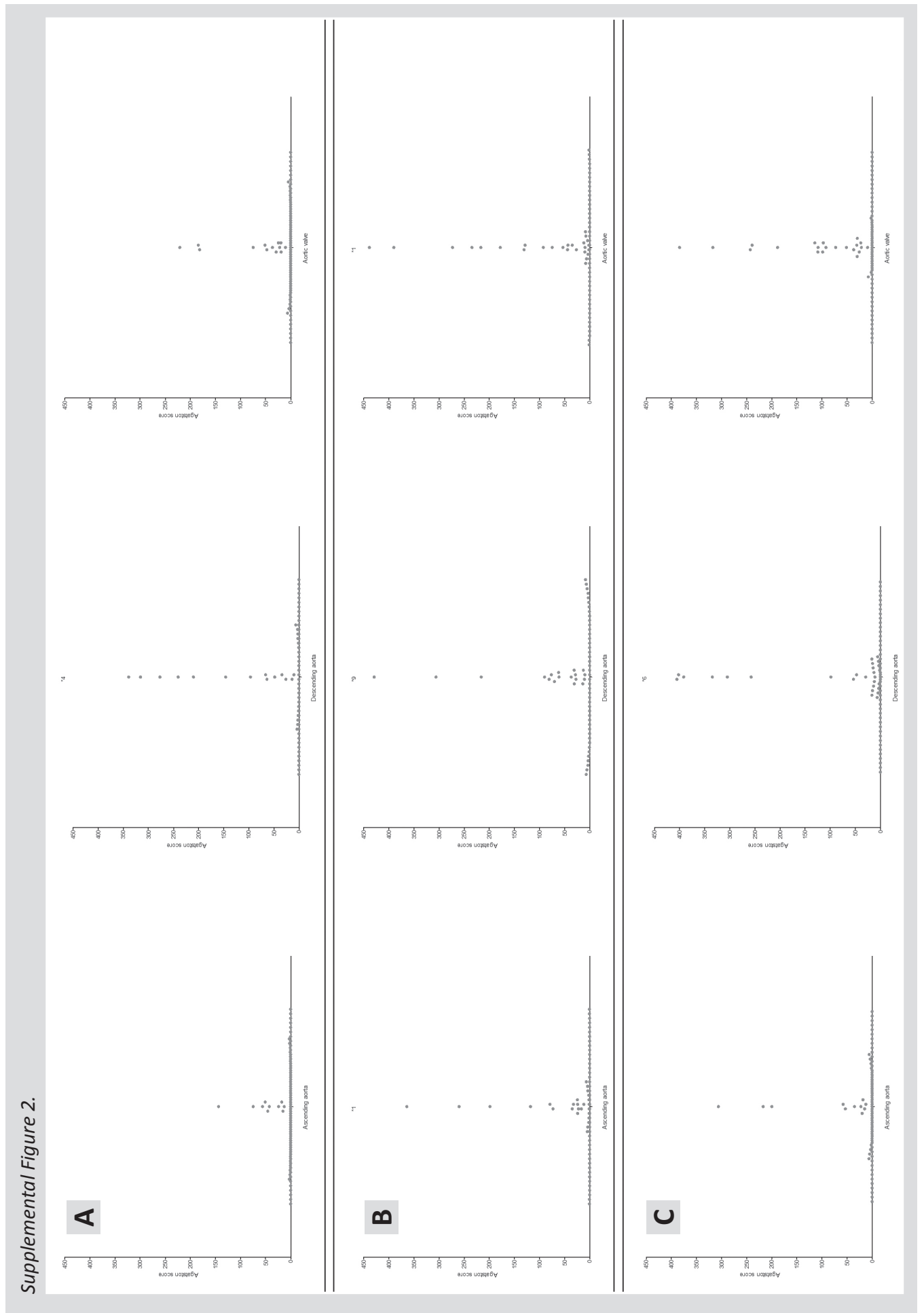

Supplemental Figure 2. Distribution of calcification (Agatston scores) in the ascending aorta, descending arta and aortic valve. A: non-OAC population. B: VKA population. C: NOAC population. ("*" provides the number of values $>450$

Abbreviations: NOAC; non-vitamin K antagonist oral anticoagulant, non-OAC; no oral anticoagulant,

VKA; Vitamin K antagonist 

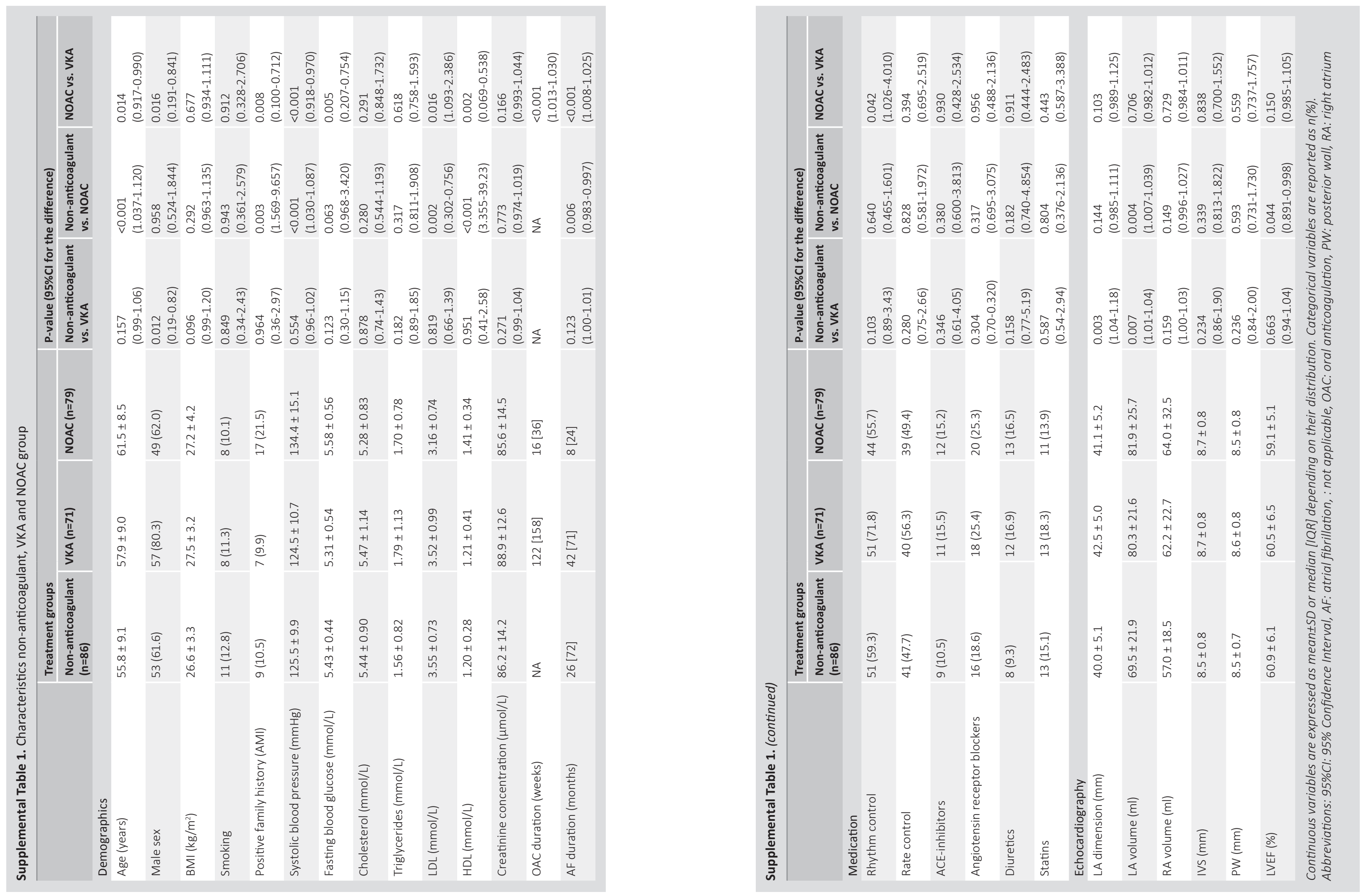

Chapter 3

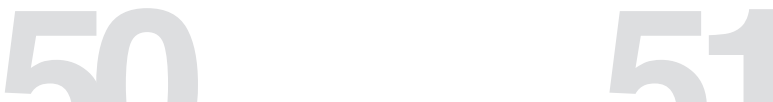



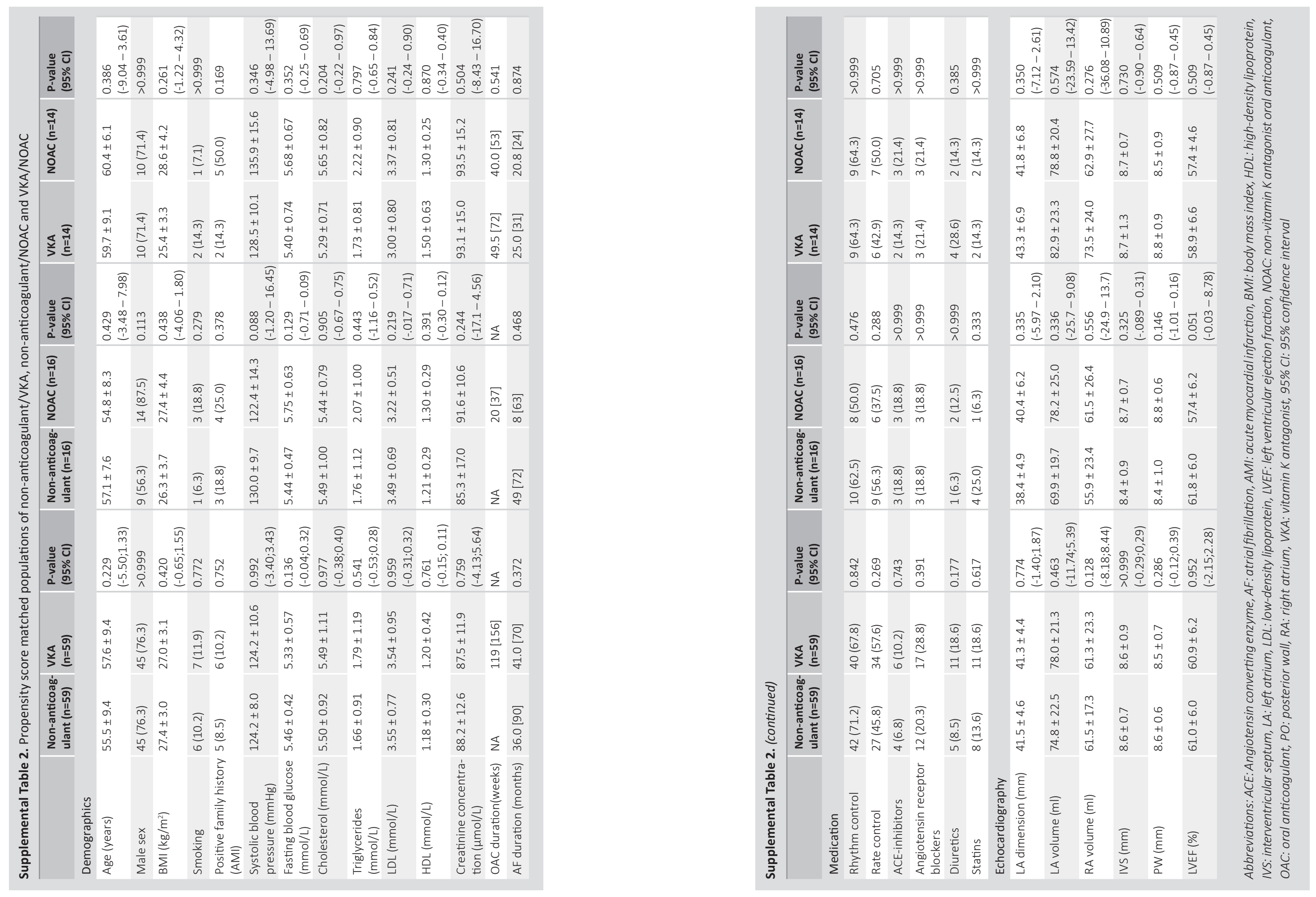


\section{Slower progress of aortic valve calcification with vitamin K supple- mentation. Results from a prospective interventional proof-of-concept study}

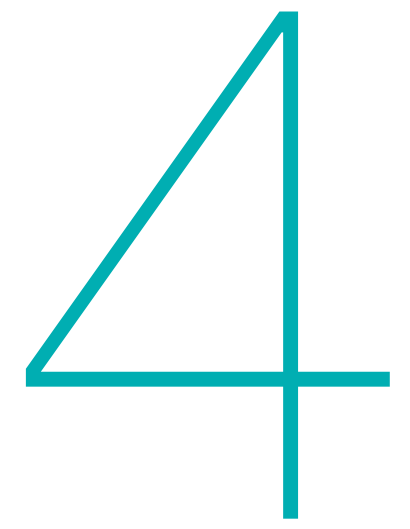

Vincent M. Brandenburg ${ }^{1}$, Sebastian Reinartz ${ }^{2}$, Nadine Kaesler ${ }^{3}$, Thilo Krüger ${ }^{3}$, Tim Dirrichs ${ }^{2}$, Rafael Kramann ${ }^{3}$, Frederique E.C.M. Peeters ${ }^{4}$, Jürgen Floege ${ }^{3}$, Andras Keszei ${ }^{5}$, Nikolaus Marx ${ }^{1}$, Leon J. Schurgers ${ }^{6}$, Ralf Koos ${ }^{1}$

${ }^{1}$ University Hospital of Rheinisch-Westfälische Technische Hochschule Aachen, department of Cardiology, Aachen, Germany,

${ }^{2}$ University Hospital of Rheinisch- Westfälische Technische Hochschule Aachen, department of Radiology, Aachen, Germany,

${ }^{3}$ University Hospital of Rheinisch- Westfälische Technische Hochschule Aachen, department of Nephrology, Aachen, Germany,

${ }^{4}$ Maastricht University Medical Center+ and CARIM, department of Cardiology, Maastricht, the Netherlands,

${ }^{5}$ University Hospital of Rheinisch- Westfälische Technische Hochschule Aachen, department of Medical Informatics, Aachen, Germany,

${ }^{6}$ Maastricht University and CARIM, department of Biochemistry, Maastricht, the Netherlands. 
Calcific aortic stenosis is a common degenerative disease characterized by progressive aortic valve calcification (AVC). ${ }^{1}$ Effective medical treatment options to retard the progression of AVC are sparse. ${ }^{1}$ Epidemiological data point to vitamin $\mathrm{K}$ as a potential protective factor for cardiovascular health, particularly for protection against vascular calcification., ${ }^{2,3}$ Matrix Gla-protein (MGP), a potent inhibitor of cardiovascular calcification, requires vitamin $\mathrm{K}$ for posttranslational carboxylation and hence full bioactivity. ${ }^{4}$ Thus, vitamin $\mathrm{K}$ supplementation might retard the progression of AVC.1,2 Dephosphorylated undercarboxylated MGP (dp-ucMGP) serves as a circulating marker for vitamin $\mathrm{K}$ deficiency. ${ }^{2,3}$

We performed a 12-month prospective, single-center, open-label, randomized interventional trial in patients with asymptomatic or mildly symptomatic AVC. Written informed consent was obtained before inclusion in the trial (URL: http://www.clinicaltrials.gov, Unique identifier: NCT00785109; RWTH Aachen Institutional Review Board No. 165/08). Inclusion criterion was peak flow velocity exceeding $2 \mathrm{~m} / \mathrm{s}$. The main exclusion criteria were chronic kidney disease estimated glomerular filtration rate $<60 \mathrm{~mL} / \mathrm{min}^{-1} / 1.73 \mathrm{~m}^{-2}$ ), expected valve replacement within the next year, and anticoagulation with vitamin $\mathrm{K}$ antagonists. Patients were randomized 1:1 to receive $2 \mathrm{mg}$ phytomenadione (vitamin K1, Ka-vit, INFECTOPHARM Arzneimittel CONSILIUM $\mathrm{GmbH}$, Heppenheim, Germany) or matching placebo once daily orally. Patients underwent baseline and end-of-study cardiac computed tomography (CT) scan for AVC quantification (volume calcification score). All CT examinations were performed on a 128-slice dual-source CT scanner (SOMATOM Definition Flash, Siemens, Germany) and were reanalyzed in a blinded fashion by 2 radiologists experienced in cardiac $\mathrm{CT}$. The primary end point was the difference in progression of AVC volume score between vitamin $\mathrm{K}$ and placebo. We also assessed changes of dp-ucMGP plasma levels (IDS, Boldon, UK) as a secondary end point. Linear regression models for AVC change with treatment effect and baseline measures were used as independent variables, and $95 \%$ confidence intervals for treatment effects were calculated.

The trial cohort included 99 patients ( $82 \%$ male; $35 \%$ with aortic sclerosis $[\leq 2.5 \mathrm{~m} / \mathrm{s}], 38 \%$ with mild aortic stenosis $[2.6-2.9 \mathrm{~m} / \mathrm{s}]$, and $27 \%$ with moderate aortic stenosis $[3.0-4.0 \mathrm{~m} / \mathrm{s}] ; 71 \%$ of each group received statins). Seventy-two participants also underwent an end-of-study cardiac CT scan (representing the per-protocol analysis cohort: $n=38$ vitamin $K, n=34$ placebo). Twenty-seven patients (12 vitamin K, 15 placebo) dropped out of the study. Reasons for discontinuation wer initiation of oral anticoagulant treatment ( $n=3$ placebo, $n=4$ vitamin $K$ ), loss to follow-up, withdrawa of consent ( $n=6$ placebo, $n=3$ vitamin $K$ ), cardiac surgery ( $n=2$ each), death ( $n=1$ each), or other reasons ( $n=3$ placebo, $n=2$ vitamin $K$ ).

Over the 12-month period, the AVC volume score progressed by $10.0 \%$ in patients in the vitamin $\mathrm{K}$ group compared with $22.0 \%$ in the placebo group (Table 1), representing a significan attenuation of AVC progression by vitamin K compared with placebo. Linear regression with treatment group and baseline AVC as independent variables revealed an estimated difference in the change in AVC volume score between the vitamin $\mathrm{K}$ and placebo groups of $-101 \mathrm{~mL}(95 \%$ confidence interval, -194 to $-8.3 ; P=0.03$, adjusted $R^{2}=0.08$ ). Adding age to the model did no improve the model or change the estimated difference. Baseline mean gradient and peak flow velocity were highly correlated $(r=0.88)$. After adjustment for mean gradient, the estimated difference in AVC volume progression was -65 (95\% confidence interval, -147 to 17 : $P=0.12$ adjusted $\mathrm{R}^{2}=0.26$ ). Similar results were obtained after AVC was indexed to body surface area. Plasma dpucMGP concentration significantly decreased in the vitamin $\mathrm{K}$ group by $45 \%(P<0.001$ Table 1). Statistically, the change in peak flow velocity was not significantly different between the 2 groups. No thromboembolic events occurred.
Table 1. Iongitudinal development of valvular calcification and echocardiographic parameters and Matrix Gla Protein levels, by intervention group

\begin{tabular}{|c|c|c|c|c|c|c|c|c|c|c|}
\hline & \multicolumn{5}{|c|}{ Vitamin K group ( $n=38$ ) } & \multicolumn{5}{|c|}{ Placebo group ( $n=34$ ) } \\
\hline & \multicolumn{2}{|l|}{ TO } & \multicolumn{2}{|l|}{ T12 } & \multirow[b]{2}{*}{ P-value } & \multicolumn{2}{|l|}{ TO } & \multicolumn{2}{|l|}{$\mathrm{T} 12$} & \multirow[b]{2}{*}{ P-value } \\
\hline & Mean & SD & Mean & SD & & Mean & SD & Mean & SD & \\
\hline Age, years & 69.3 & 10.1 & & & & & & 68.9 & 7.9 & \\
\hline $\begin{array}{l}\text { Calcification volume } \\
(\mathrm{mL})\end{array}$ & 793 & 742 & 871 & 791 & $0.006^{*}$ & 836 & 856 & 1017 & 939 & $<0.001^{\dagger}$ \\
\hline $\begin{array}{l}\text { Mean valvular } \\
\text { gradient (mmHg) }\end{array}$ & 17 & 6 & 17 & 7 & $0.63^{*}$ & 18 & 8 & 21 & 11 & $0.03^{+}$ \\
\hline $\begin{array}{l}\text { Peak flow velocity } \\
(\mathrm{m} / \mathrm{s})\end{array}$ & 2.70 & 0.44 & 2.63 & 0.56 & $0.42^{*}$ & 2.82 & 0.62 & 2.95 & 0.70 & $0.24 \dagger$ \\
\hline Dp-ucMGP, pmol/L & 432 & 149 & 243 & 165 & $<0.001^{*}$ & 483 & 215 & 515 & 276 & $0.10^{+}$ \\
\hline $\begin{array}{l}\Delta \text { calcification } \\
\text { volume }(\mathrm{mL})\end{array}$ & & & 78 & 165 & & & & 181 & 234 & $0.04 \ddagger$ \\
\hline $\begin{array}{l}\Delta \text { calcification } \\
\text { volume index } \\
\left(\mathrm{mL} / \mathrm{m}^{2} \mathrm{BSA}\right)\end{array}$ & & & 41 & 84 & & & & 91 & 113 & $0.04 \ddagger$ \\
\hline $\begin{array}{l}\text { Delta dp-ucMGP } \\
\text { (pmol/L) }\end{array}$ & & & -199 & 233 & & & & 37 & 112 & $<0.001 \neq$ \\
\hline $\begin{array}{l}\text { Delta peak flow } \\
\text { velocity }(\mathrm{m} / \mathrm{s})\end{array}$ & & & -0.05 & -0.38 & & & & 0.09 & 0.40 & $0.16 \neq$ \\
\hline
\end{tabular}

Abbreviations: BSA: body surface area; dp-ucMGP, dephosphorylated undercarboxylated matrix Gla-protein $S D$ : standard deviation

*Nominal P values from paired comparison between baseline (TO) and 12 months (T12) in the vitamin $K$ group

tNominal P values from paired comparison between TO and T12 in the placebo group

Fominal P values from unadjusted, unpaired comparison of delta values between treatment groups

The present study is the first randomized controlled trial in men to demonstrate that vitamin $\mathrm{K}$ supplementation might decelerate the progression of AVC. Our findings are clinically meaningfu because a strong, significant correlation exists between the AVC volume score and functional valvular parameters such as mean gradient or peak flow velocity. ${ }^{5}$ Hence, deceleration of AVC progression, a direct precursor of hemodynamic impairment, might finally translate into a stabilization of valvular functionality in calcific aortic stenosis and a slowing of cardiac and clinical deterioration. In parallel, vitamin K treatment induced a marked reduction of plasma dpucMGP, indicating increased vitamin $\mathrm{K}$ bioactivity.

We consider the present study results to represent the first proof of concept in the evaluation of the potential anti-calcification effects of vitamin $\mathrm{K}$ treatment in human calcific aortic valvular disease. We acknowledge that our results need to be confirmed and should therefore be interpreted with caution. Limitations of our trial are the relatively small study size and the additional high dropout rate, resulting in missing data for primary end-point interpretation, as well as the shor duration of follow-up, the open-label design, and the broad spectrum of severity of valvular disease at baseline. Moreover, the study was not powered to assess valve functionality using 
echocardiography, an important determinant for clinical end points. Despite these limitations, our data lay the basis for future intervention trials to investigate valvular hemodynamic parameters or patient outcomes in parallel to calcification parameters.

In summary, vitamin K supplementation may represent an effective and safe therapy in cardiovascular disease related to ectopic calcification such as calcific aortic stenosis.

\section{REFERENCES}

G, Redfors B, Leon MB, G.n.reux P. Medica stenosis. Circulation. 2016;134:1766-

. Brandenburg VM, Schurgers LJ, Kaesler N, Püsche K, van Gorp RH, Leftheriotis G, Reinartz S, Koos R, Krüger T. Prevention of vasculopathy by vitamin K supplementation: can doi: 101016/i atheroscerosis 201502040 -

The realm of vitamin $\mathrm{K}$ dependent proteins: shifting from coagulation toward calcification. Mol Nutr Food Res. 2014:58:1620-1635. doi: 10.1002/mnfr.201300743.

Cranenburg EC, Vermeer C, Koos R, Boumans ML, Hackeng TM, Bouwman $F G$, Kwaijtaal $M$, Brandenburg VM matrix Gla Protein (UC- MGP) as a biomarker for cardiovascular calcification. J Vasc Res. 2008;45:427-436. do 10.1159/000124863.

5. Koos R, Mahnken AH, Sinha AM, Wildberger JE, Hoffmann R, Kuhl HP. Aortic valve calcification as a marker for aortic

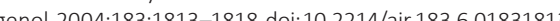




\section{Bicuspid aortic valve stenosis and the effect of vitamin K2 on calci- fication using ${ }^{18} \mathrm{~F}$-sodium fluoride positron emission tomography/ magnetic resonance: the BASIK2 rationale and trial design}

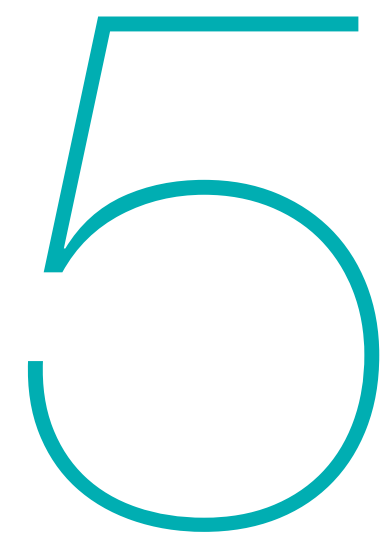

Frederique E.C.M. Peeters ${ }^{1}$, Manouk J.W. van Mourik', Steven J.R. Meex², Jan Bucerius ${ }^{3,4}$, Simon M. Schalla ${ }^{5}$, Suzanne C. Gerretsen ${ }^{3}$, Casper Mihl ${ }^{3}$, Marc R. Dweck ${ }^{6}$, Leon J. Schurgers ${ }^{7}$, Joachim E. Wildberger ${ }^{3}$, Harry J.G.M. Crijns ${ }^{1}$ and Bas L.J.H. Kietselaer ${ }^{8}$

${ }^{1}$ Maastricht University Medical Center+ and CARIM, department of Cardiology, Maastricht, the Netherlands,

${ }^{2}$ Maastricht University Medical Center+ and CARIM, department of Clinical chemistry, Maastricht, the Netherlands,

${ }^{3}$ Maastricht University Medical Center+ and CARIM, department of Radiology and Nuclear Medicine, Maastricht, the Netherlands,

${ }^{4}$ University Hospital RWTH Aachen, department of Nuclear Medicine, Aachen, Germany,

${ }^{5}$ Maastricht University Medical Center+ and CARIM, departments of Cardiology and Radiology, Maastricht, the Netherlands,

${ }^{6}$ Centre for Cardiovascular Science, University of Edinburgh, Edinburgh, UK,

${ }^{7}$ Maastricht University and CARIM, department of Biochemistry, Maastricht, the Netherlands,

${ }^{8}$ Zuyderland Medisch Centrum Heerlen/Sittard, Heerlen/Sittard, the Netherlands 


\section{ABSTRACT}

BASIK2 is a prospective, double-blind, randomized placebo-controlled trial investigating the effect of vitamin K2 (menaquinone-7; MK7) on imaging measurements of calcification in the bicuspid aortic valve (BAV) and calcific aortic valve stenosis (CAVS). BAV is associated with early developmen of CAVS. Pathophysiologic mechanisms are incompletely defined, and the only treatment available is valve replacement upon progression to severe symptomatic stenosis. Matrix Gla protein (MGP) inactivity is suggested to be involved in progression. Being a vitamin $\mathrm{K}$ dependent protein supplementation with MK7 is a pharmacological option for activating MGP and intervening in the progression of CAVS. Forty-four subjects with BAV and mild-moderate CAVS will be included in the study, and baseline ${ }^{18} \mathrm{~F}$-sodiumfluoride $\left({ }^{18} \mathrm{~F}-\mathrm{NaF}\right)$ positron emission tomography (PET)/ magnetic resonance (MR) and computed tomography (CT) assessments will be performed. Thereafter, subjects will be randomized (1:1) to MK7 (360 mcg/day) or placebo. During an 18-month follow-up period, subjects will visit the hospital every 6 months, undergoing a second ${ }^{18} \mathrm{~F}-\mathrm{NaF}$ PET/ $M R$ after 6 months and $C T$ after 6 and 18 months. The primary endpoint is the change in PET/MR ${ }^{18} \mathrm{~F}-\mathrm{NaF}$ uptake (6 months minus baseline) compared to this delta change in the placebo arm. The main secondary endpoints are changes in calcium score (CT), progression of the left ventricular remodeling response and CAVS severity (echocardiography). We will also examine the association between early calcification activity (PET) and later changes in calcium score (CT).

Key words: bicuspid aortic valve; calcific aortic valve stenosis; vitamin K2; menaquinone-7; PET/ $\mathrm{MR} ;{ }^{18} \mathrm{~F}-\mathrm{NaF}$ 


\section{TRIAL DESIGN}

The BASIK2 trial is an investigator-initiated, prospective, double blind, randomized, placebocontrolled trial, studying the effects of vitamin K2 (menaquinone-7, MK7) or placebo on the progression of calcification in CAVS using ${ }^{18} \mathrm{~F}-\mathrm{NaF}$ PET/MR in patients with a bicuspid aortic valve and calcific aortic valve stenosis. The study was approved by the institutional review board (Maastricht Academic Hospital and Maastricht University, the Netherlands: NL54600.068.015/ METC152045) and conducted according to the principles of the Declaration of Helsinki. The BASIK2 trial is registered in clinicaltrials.gov as NCT02917525. All subjects gave their written informed consent for inclusion before they participated in the study.

In subjects meeting requirements for trial participation an ${ }^{18} \mathrm{~F}-\mathrm{NaF}$ PET/MR and a non-contrast CT will be performed at baseline after providing informed consent. Furthermore, echocardiography and venipuncture will be performed. Thereafter, subjects will be randomized (1:1) to the intervention or control group, receiving an oral dose of 360 micrograms $(\mathrm{mcg})$ menaquinone-7 or placebo respectively (NattoPharma ASA, Hovik, Norway). The total study duration is 18 months, in which subjects will visit the outpatient clinic every six months. After six months, subjects will again undergo PET-MR, and uptake of ${ }^{18} \mathrm{~F}-\mathrm{NaF}$ will be quantified to assess the (difference in) active calcification of the aortic valve and the potential effect of MK7 supplementation. Furthermore, subjects will undergo a (non-contrast) CT after 6 and 18 months. Transthoracic echocardiography and venipuncture will be performed every visit during the follow-up period. Additional clinical information (such as medical history, cardiovascular risk factors, current medication, family history) will be obtained from the electronic hospital charts and will be evaluated every visit (if relevant).

The study flowchart is illustrated in Figure 1. These investigations will enable the evaluation of several effects of MK7 and (the natural course of) progression of CAVS in this population, in addition to the pre-specified primary endpoint. The total study population will consist of 44 patients.

\section{INCLUSION AND EXCLUSION CRITERIA}

A detailed overview of inclusion and exclusion criteria is provided in Table 2. In short, all patients ( $>18$ years) being followed up at the outpatient clinics of the Maastricht University Medical Center (MUMC+) with a bicuspid aortic valve (BAV), mild to moderate aortic valve stenosis and calcification confirmed on echocardiography will be screened for eligibility. The presence of BAV will be confirmed using short-axis echocardiographic images and morphology will be determined during systole [26]. Patients who meet any of the exclusion criteria (including standard contra-indications for MR) and those unable to provide written informed consent will not be included.

\section{STUDY OBJECTIVES AND STATISTICAL ANALYSES PLAN}

\section{Primary endpoints and sample size calculation}

The central aim of the current trial is to assess whether supplementation with menaquinone-7 wil slow or even reverse aortic valve calcification activity. Therefore, the primary endpoint is the change in ${ }^{18} \mathrm{~F}-\mathrm{NaF}$ tracer uptake on ${ }^{18} \mathrm{~F}-\mathrm{NaF}$ PET/MR (6 months minus baseline).

At the time that the current study was designed, literature reporting the specified treatment effect in similar studies was sparse. Therefore, the sample size calculation was based on expected changes in CT calcium scores at the secondary endpoint. ${ }^{22,27,28}$ The mean annual calcification progression on $\mathrm{CT}$ has been estimated to be $21.7 \%$, with a standard deviation of $19.8 \% .^{23}$

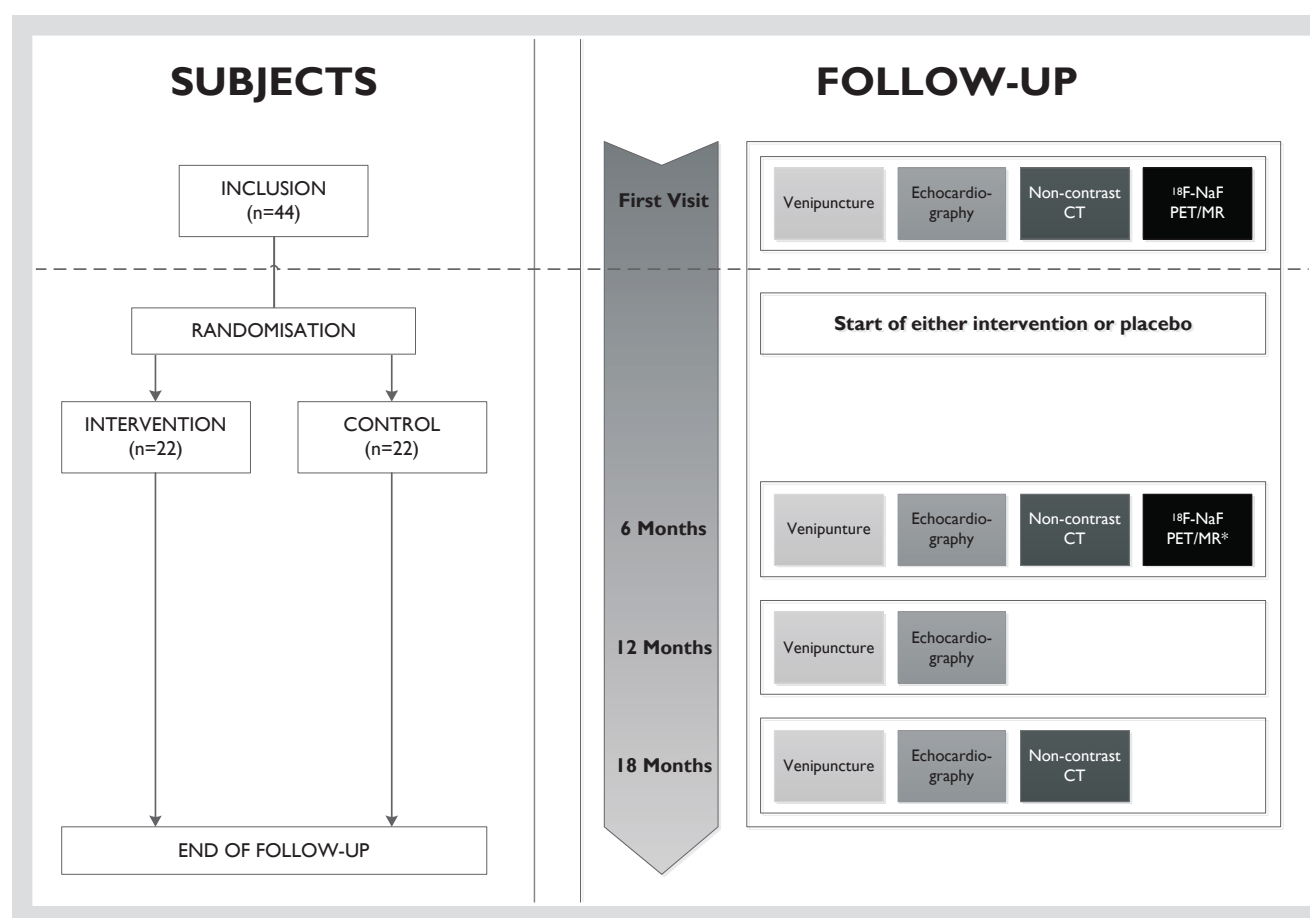

Figure 1. Study flowchart.* Primary endpoint (change from baseline in tracer uptake in the aortic valve by ${ }^{18} \mathrm{~F}$-sodiumfluoride ${ }^{18} \mathrm{~F}$-NaF) positron emission tomography (PET)/ magnetic resonance (MR) at 6 months). Abbrevations: ${ }^{18} \mathrm{~F}-\mathrm{NaF}$; ${ }^{18} \mathrm{~F}$-sodiumfluoride, CT; computed tomography, MR: magnetic resonance, PET; positron emission tomography

Considering these premises, the variability of the calcification progression is estimated to be comparable to the standard deviation mentioned above (19.8\%). An absolute difference in calcification progression of $20 \%$ between the groups is considered a significant effect. With a significance level alpha of 0.05 , a power of $80 \%$ and an estimated dropout of approximately $25 \%$, 44 patients will be required to detect a difference between the treatment groups ( $\sim 22$ subjects each). ${ }^{29}$ This power calculation is conservative since change in CT calcium score is considered les sensitive than changes in ${ }^{18} \mathrm{~F}-\mathrm{NaF}$ uptake, ${ }^{18}$ and the calculated number of individuals is expected to afford more power to demonstrate a treatment effect for the primary endpoint $\left({ }^{18} \mathrm{~F}-\mathrm{NaF}\right.$ uptake). ${ }^{18} \mathrm{~F}-\mathrm{NaF}$ tracer uptake was shown to be present in patients with CAVS in regions overlying, adjacent to and remote from existing valvular calcification, ${ }^{30}$ and a recent study provided the first preliminary evidence that ${ }^{18} \mathrm{~F}-\mathrm{NaF}$ is a very sensitive marker of progression of aortic valve disease. ${ }^{18}$ The six month time window between baseline and follow up measurement with molecular PET imaging is also rather conservative and was derived from earlier studies investigating the effect of short-term statin therapy on vascular inflammation and calcification on fluorodeoxyglucose (FDG) PET/CT, showing a significant reduction in tracer uptake after 3 months of treatment. ${ }^{31,32}$ 

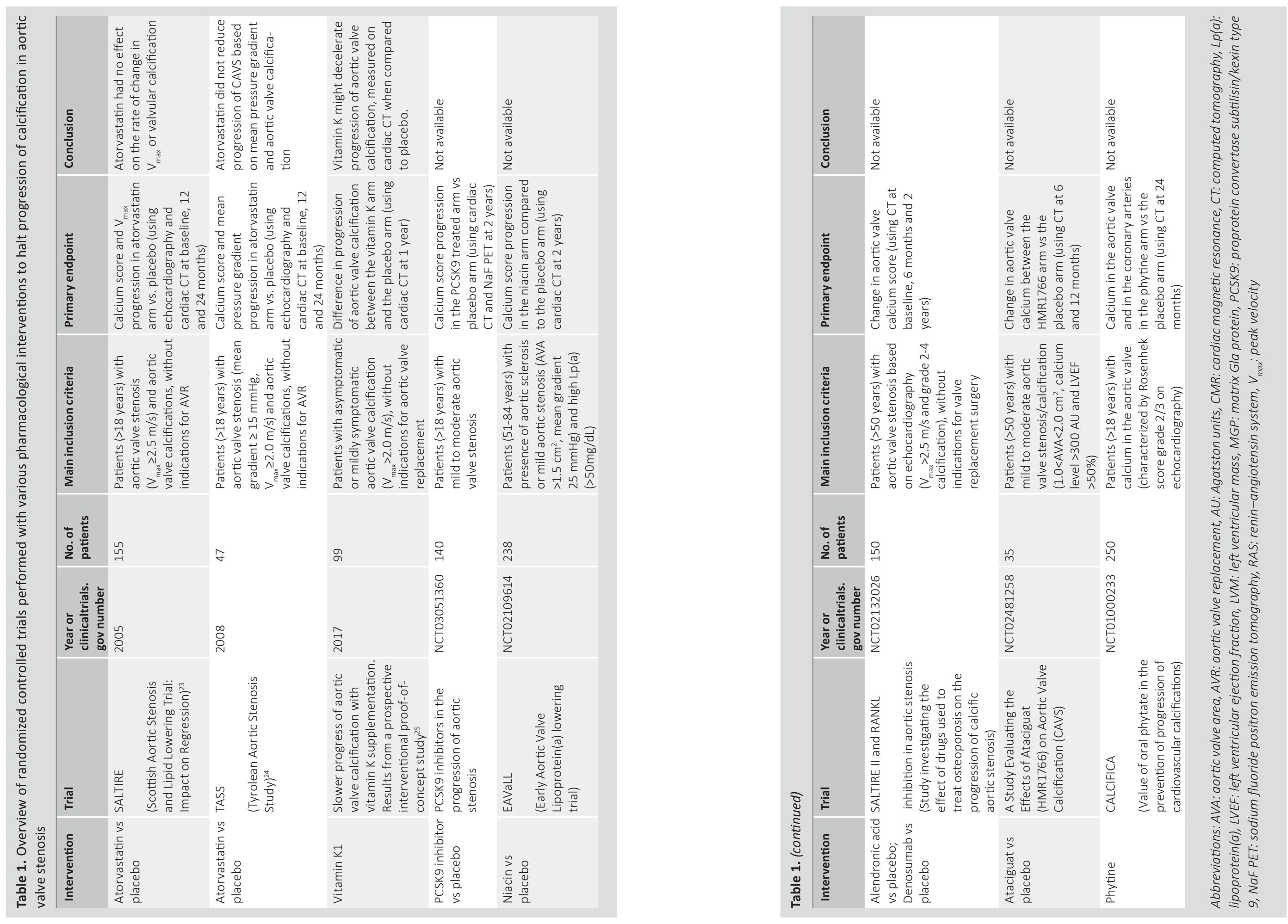

Chapter 5 


\section{Table 2. Eligibility criteria}

\section{Inclusion criteria}

Age $>18$ years

Presence of bicuspid aortic valve

Calcified mild to moderate aortic valve stenosis (mean gradient $<40 \mathrm{mmHg}$, maximum gradient between 25-64 $\mathrm{mmHg}$ or $\mathrm{V}_{\max }$ between $2.5-4 \mathrm{~m} / \mathrm{s}$ )

\section{Exclusion criteria}

Absence of bicuspid aortic valve

Absence of calcified aortic valve stenosis (mean gradient $<10 \mathrm{mmHg}$, Vmax $<2.5 \mathrm{~m} / \mathrm{s}$ or AVA $3-4 \mathrm{~cm}^{2}$ ) Presence of severe aortic valve stenosis (mean gradient $>40 \mathrm{mmHg}$, maximum gradient $>64 \mathrm{mmHg}$ or AVA $\left.<1.0 \mathrm{~cm}^{2}\right)$

Aortic valve replacement or repair (scheduled)

Accepted atrial fibrillation

Use of vitamin $\mathrm{K}$ antagonists

Malignant disease $<2$ years (except non-melanoma skin cancer, or in situ carcinoma of the cervix)

Life expectancy $<2$ years

Present pregnancy or wish for near future pregnancy

Claustrophobia

Metallic implant (neurostimulator, cochlear implant, vascular clip)

Pacemaker or ICD

Adipositas per magna

Abbreviations: AVA: aortic valve area; ICD: implantable cardiac defibrillator; $V_{\max }$ : peak jet velocity.

Since it is known that progression of aortic valve stenosis is not a linear process, but rather shows a trend towards an increasing progression rate in advanced disease,,$^{33}$ patients with less than mild aortic valve stenosis and patients with severe calcified aortic valve stenosis at baseline will be excluded. Patients with a bicuspid aortic valve have an increased risk for aortic valve replacement from approximately the fourth decade in life, ${ }_{,}^{4}$ suggesting a more rapid rate of progression, possibly due to altered hemodynamic circumstances. ${ }^{5}$

\section{Secondary endpoints}

Secondary objectives include the following: change from baseline in calcium score of the aortic valve measured by CT after 6 and 18 months, the correlation between tracer uptake after 6 months and calcium score by $\mathrm{CT}$ after 18 months, change from baseline in echocardiographic parameters depicting the progression of CAVS, the response of left ventricular (LV) function to CAVS on echocardiography, and the change from baseline in left ventricular function, left ventricular mass, aortic distensibility and aortic flow on MR after 6 months. Furthermore, the LV response to aortic valve stenosis, as measured by changes in resting cardiac troponin and NT-proBNP will be investigated, as well as associations between changes in biomarkers and progression of calcification after 6,12 and 18 months.

Data from all included patients will be used for the analyses. In the case of exclusion of a patient during the trial, data from the patient will be collected and included in the analyses up unti their exclusion.

\section{Additional analyses}

The main outcome parameter (change in calcification activity between the MK7-treated arm an placebo) will be presented as a continuous variable. The mean difference in calcification activity between treatment arms will be expressed as the difference between ${ }^{18} \mathrm{~F}-\mathrm{NaF}$ uptake during follow-up and ${ }^{18} \mathrm{~F}-\mathrm{NaF}$ uptake at baseline. The $\mathrm{CT}$ calcium score will be presented as a continuous variable, and the calcification (score) progression will be expressed as the mean difference (calcium score (aortic valve) during follow-up minus calcium score (aortic valve) at baseline) and will be presented as a dichotomous variable (rapid progression and slow progression).

Data will be analyzed based on the intention-to-treat principle. Baseline and follow-up categorical variables will be expressed as percentages and continuous variables as means t standard deviations. The independent $t$-test or Mann-Whitney $U$ test will be used to test differences between normally-distributed continuous variables and continuous variables not showing a normal distribution, respectively. A paired $t$-test or Wilcoxon signed rank test will be applied when appropriate. Categorical variables will be tested using the Fisher's exact or Chi square test. A two-sided significance level of $5 \%$ will be considered to be statistically significant.

Univariate analysis and multiple regression analysis will be used to investigate the existence of significant predictor(s) for the outcome variable-calcification progression.

\section{STUDY PROCEDURES}

PET and MR imaging

Combined PET/MR scans will be performed at inclusion and after 6 months of follow-up using full-integrated Tesla PET-MR scanner (Siemens Biograph Mmr $r^{\top \mathrm{M}}$, Siemens Healthineers, Forchheim Germany). A dose of $185 \mathrm{MBq}$ of NaF will be injected intravenously. After $30 \mathrm{~min}$, (non-contrast) MR scanning will be started. PET data acquisition will be started 60 min after intravenous administration of the radiopharmacon. Dixon-based MR images will be used for attenuation correction.

Heart and large vessel anatomy will be determined using a T1-weighted black blood sequence (transversal and oblique sagittal plane) turbo spin echo sequence, prospectively triggered (average repetition time (TR)/echo time (TE): $740 \mathrm{~ms} / 27 \mathrm{~ms}$, resolution $1.3 \times 1.3 \times 8.0 \mathrm{~mm}$ ). Cine-MR views of the heart in the horizontal and vertical long axes, short axes and left ventricular outflow tract will be acquired according to standard clinical protocols to obtain ventricular volume, mass and function, and $3-5$ slices of cross sectional cine images at the level of the aortic root will be acquired to obtain valvular anatomy and function (all cine images: balanced fast field echo sequence, retrospectively triggered. TR/TE/flip angle: $41.28 \mathrm{~ms} / 1.51 \mathrm{~ms} / 50^{\circ}$, resolution $1.3 \times 1.3 \times 8.0 \mathrm{~mm}$ ). Flow imaging will be performed at the level of the aortic valve and the ascending aorta. Analyses of source images will be performed using dedicated software (Syngo.via ${ }^{T M}$, Siemens Healthineers, Forchheim, Germany). PET signal quantification will be performed by delineating regions of interest (ROI) using both PET and MR images. Moreover, (non-contrast) CT images will be used to localize regions of macrocalcification.

\section{Computed Tomography (CT) imagin}

A breath-held, non-contrast, enhanced CT scan will be performed at inclusion and during the visits at 6 and 18 months of follow-up to determine calcification of the aortic valve and the thoracic aorta. These scans will be performed using a third generation, dual-source CT-scanner (Somatom Definition Force, Siemens Healthineers, Forchheim, Germany). The scan protocol for calcium 
scoring will be performed at a tube voltage of $120 \mathrm{kV}$, reference quality tube current of $80 \mathrm{mAs}$, $2 \times 192 \times 0.6 \mathrm{~mm}$ collimation, a gantry rotation time of $0.25 \mathrm{~s}$ and a pitch value of 3.2. Calcification quantification (mass, volume and score) of the aortic valve and the thoracic aorta will be determined using dedicated post-processing software (Syngo.via, Siemens Healthineers, Forchheim, Germany). ${ }^{27}$ Calcium localized from the sinotubular junction to the end of scan range, or up to the origin of the brachiocephalic artery, is considered to be in the ascending aorta. Calcium present distal from the origin of the left subclavian artery up to the diaphragm is considered to be localized in the descending aorta. Quantification will be performed by two observers, both blinded to medical data. In the case of ambiguity, consensus will be reached by discussion/in the presence of a third observer.

\section{Echocardiography}

Transthoracic echocardiographic examinations will be performed every 6 months. All parameter (presented in Table 3) are part of the regular echocardiographic examination and will be assessed according to EAE/ASE guidelines.

\section{Table 3. Echocardiographic parameters}

Anatomy and function $\mathrm{AoV}^{34-36}$

Diameter LVOT, aortic sinus, STJ, ascending aorta

Systolic LV function and dimension ${ }^{37-38}$

Filling pressure and LV diastolic function ${ }^{39}$

RV function ${ }^{40}$

Abbreviations: AoV: aortic valve, LV: left ventricle, LVOT: left ventricular outflow tract, RV: right ventricle, STJ: sinotubular junction

\section{Laboratory assessments}

Blood sampling will be conducted by standard venipuncture during all study visits. Standard hematological parameters (hemoglobin, hematocrit, thrombocytes, leucocytes) and differentiation will be evaluated. Additional samples will be stored at $-80{ }^{\circ} \mathrm{C}$ for future biomarker analyses investigating kidney function, vitamin $\mathrm{K}$ status and calcification inhibitor concentrations over time. Moreover, the association between biomarkers and calcification/aortic valve stenosis, left ventricular response and (long term) diastolic function will be investigated in future analyses.

\section{Randomization and study intervention}

Subjects will be randomized after the initial scans in a 1:1 fashion, by an independent investigator to the (1) intervention group (MK7) or (2) placebo group (Figure 1). Block randomization (4 or 6 subjects per block) will be assembled to safeguard equal allocation of subjects to the treatment groups.

\section{Study intervention}

Patients in the intervention group will receive a capsule containing $360 \mathrm{mcg}$ of menaquinone-7 (MK7, NattoPharma ASA, Oslo, Norway) daily for 18 months. Capsules consist of synthetic MK7 (bioequivalent to soy and natural chickpea MenaQQ7). ${ }^{41}$ The choice to use MK7 is based on its longer half-life and its favorable extra-hepatic distribution compared to other forms of vitamin K2. ${ }^{16}$ The dose to be used in this trial was established in a dose-finding study, in which a positive dose-dependent effect of menaquinone-7 on MGP- and osteocalcin-carboxylation was found. Non-functional MGP was decreased most effectively using a daily dose of $360 \mathrm{mcg}$ MK7.42-44 Furthermore, MK7 does not cause a hypercoagulable state and is well-tolerated. ${ }^{45}$

The placebo capsule does not differ from the MK7 capsule with regard to shape, taste and additives, but does not contain MK7.

Patients receive a pre-specified number of tablets at each visit. The next visit, patients will hand the leftover tablets to the investigator who will provide the patient with the next pre-specified number of tablets. Compliance will be monitored at each visit by performing and registering a pill count. Furthermore, vitamin $\mathrm{K}$ status and concentration of dephosphorylated uncarboxylated MGP (dp-ucMGP) over time will be determined at the end of the study.

\section{SUMMARY}

The BASIK2 study is a proof of concept trial that will provide us with information on calcium activity in the aortic valve and the potential effect of supplementation with vitamin K2 (more specifically; MK7). This trial bears the potential to open novel avenues for future large scale randomized controlled trials to intervene in the progression of CAVS. 


\section{REFERENCES}

Siu, S.C.; Silversides, C.K. Bicuspid ao
Am. Coll. Cardiol. 2010, 55, 2789-2800. Go, A.S.; Arnett, D.K.; Blaha, M.J.; Cushman, M.; Das, S.R. de Ferranti, S.; Despres, J.P., et al. Heart Disease and Stroke
Statistics-2016 Update: A Report From the American Heart Association. Circulation 2016, 133, e38-e360.

Hutcheson, J.D.; Aikawa, E.; Merryman, W.D. Potential drus targets for calcific

4. Roberts, W.C.; Ko, J.M. Frequency by decades of unicuspid, bicuspid, and tricuspid aortic valves in adults having isolated aortic valve replacement for aortic stenosis, with or Culation 2005,

contributiondra, S.; Sucosky, P. Ex vivo evidence for the to the early pathogenesis of calcific bicuspid aortic valve disease. PLOS ONE 2012, 7, e48843,

New, S.E.; Aikawa, E. Molecular imaging insights into early inflammatory stages of arterial and ar. Res. 2011, 108, 1381-1391.

Otto, C.M. Kuusisto, J. Reichenbach, D.D. Gown AM O'Brien, K.D. Characterization of the early lesion of 'dege erative' valvular aortic stenosis. Histological and immun-
histochemical studies. Circulation 1994, 90, 844-853.

8. histochemical studies. Circulation $1994,90,844-853$. Pawade, T.A.; Newby, D.E.; Dweck, M.R. Calcification in
Aortic Stenosis: The Skeleton Key. I. Am. Coll. Cardiol. 2015, 66, 561-577.

9. Rajamannan, N.M.; Evans, F.J; Aikawa, E.; Grande-Allen K.J.; Demer, L.L.; Heistad, D.D.; Simmons, C.A. Masters, K.S.; Mathieu, P.; O'Brien, K.D.; et al. Calcific aortic valve disease: Not simply a degenerative process: A review and agenda for research from the National Heart and Lung and 2 (a) summary: Calcific aortic valve
lation 2011, 124, 1783-1791.

10. Schurgers, L.J.; Vitto, J.; Reutelingsperger, C.P. Vitamin $K$-dependent carboxylation of matrix Gla-protein: A crucia switch to control ectopic mineralization. Irends $\mathrm{Mol}$. Med

11. Price, P.A.; Thomas, G.R.; Pardini, A.W.; Figueira, W.F.; lar weight complex of calcium, phosphate, fetuin, and matrix gamma-carboxyglutamic acid protein in the serum of etidronate-treated rats. J. Biol. Chem. 2002, 277, Venardos, $N$

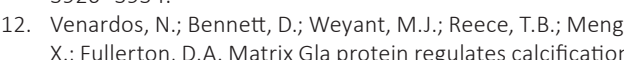
of the aortic valve. J. Surg. Res. 2015, 199, 1-6. 13. Gast, G.C.; de Roos, N.M.; Sluijs, I.; Bots, M.L.; Beulens, J.W.
Geleijnse, J.M.; Witteman, J.C.; Grobbee, D.E.; Peeters, P.H. van der Schouw, Y.T. A high menaquinone intake reduces
the incidence of coronary heart disease. Nutr. Metab. Carthe incidence of coronary heart disease.
diovasc. Dis. NMCD 2009, 19, 504-510.

14. Geleijnse, J.M.; Vermeer, C.; Grobbee, D.E.; Schurgers, L.J Knapen, M.H.; van der Meer, I.M.; Hofman, A.; Witteman, .C. Dietary intake of menaquinone is associated with reduced risk of coronary heart dis
Study. J. Nutr. 2004, 134, 3100-3105.

5. Schurgers, L.J.; Vermeer, C. Determination of phylloqu none and menaquinones in food. Effect of food matrix on 30, 298-307.
Schurgers, L.J.; Teunissen, K.J.; Hamulyak, K.; Knapen, M.H. Vik, H.; Vermeer, C. Vitamin K-containing dietary supplements: Comparison of synthetic vitamin K1 and nattoA. Dweck, M.R.; Joshi, F.R.; Gallagher, F.A.; Warburton, E.A. Bennett, M.R.; et al. Identifying active vascular microcalcification by (18)F-sodium fluoride positron emission tomegrphy. Nat. Commun. 2015, 6, 7495 C.W.; Malley, T.S.; Cowie, W.J.; Tsampasian, V.; Richardson, H.; marker of active calcification and disease progression patients with aortic sten

Jenkins, W.S.; Vesey, A.T.; Shah, A.S.; Pawade, T.A.; Chin, C.W.; White, A.C.; Fletcher, A.; Cartlidge, T.R.; Mitchell, A.J.
Pringle, M.A.; et al. Valvular (18)F-Fluoride and (18) F-Fluorodeoxyglucose Uptake Predict Disease Progressio and Clinical Outcome in Patients With Aortic Stenosis. Am. Coll. Cardiol. 2015, 66, 1200-1201.

PET/MRI:Challenges and Opportunities. Cardiol Clin. 2016, $34,25-35$.

Ratib, O.; Nkoulou, R. Potential Applications of PET/MR Imasing in Cardiology. J. Nucl. Med. 2014, 55 (Suppl. 2), 40S-465. Baumgartner, H., Falk, V., Bax, J.J., De Bonis, M., Hamm, C. Holm, P.., lung, B., Lancellott, ,., Lansac, E., M Mnoz, D. R. valvular heart disease: The Task Force for the Management of Valvular Heart Disease of the European Society of Cardology (ESC) and the European Association for Cardio-Thoracic Surgery (EACTS). Eur. Heart J. 2017, 38, 2739-2791. Cowell, S.J.; Newby, D.E.; Prescott, R.J.; Bloomfield, P.; Reid, Lowering Trial, Impact on Regression (SALTIRE) Investigators. A randomized trial of intensive lipid-lowering therapy in cacific aortic stenosis. N. Engl. J. Med. 2005, 352, 2389-2397. Dichtl, W.; Alber, H.F.; Feuchtner, G.M.; Hintringer, F.; Reinthaler, M.; Bartel, T.; Sussenbacher, A.; Grander, W.; Ulmer H., Pachinger, $\mathrm{O}$, ; et al. Prognosis and risk factors in patient torvastatin $(20 \mathrm{mg}) . \mathrm{m}$. Cardio $2008,102,743-748$ Brandenburg, V.M.: Reinartz, S.; Kaesler, N. Kruger, T. Dirrichs, T.; Kramann, R.; Peeters, F.; Floege, J.; Keszei, A.; Marx, N.; et al. Slower Progress of Aortic Valve Calcification With Vitamin K Supplementation: Results From a Prospective Interventional Profof Concen $S$

C. A classification system for the Cardiovasc. Surg. 2007, 133, 1226-1233.

Agatston, A.S., Janowitz, W.R.; Hildner, F.J.; Zusmer, N.R. arery .

Messika-Zeitoun, D.; Bielak, L.F; Peyser, P.A.; Sheedy, P.F. Tajik, A.J.; et al. Aortic valve calcification: Determinants an progression in the population. Arterioscler. Thromb. Vas Biol. 2007, 27, 642-648.

Noordzij, M.; Tripepi, G.; Dekker, F.W.; Zoccali, C.; Tanck, M.W. mon pitfalls. Nephrol. Dial. Transplant. 2010, 25, 1388-1393.
30. Dweck, M.R.; Jones, C.; Joshi, N.V.; Fletcher, A.M.; Richardson, H.; White, A.; Marsden, M.; Pessotto, R.; Clark, J.C. Wallace, W.A., et al. patients with aortic stenosis. Circulation 2012, 125,76-86.

31. Tahara, N.; Kai, H.; Ishibashi, M.; Nakaura, H.; Kaida, H.; Baba, K.; Hayabuchi, N.; Imaizumi, T. Simvastatin attenuates plaque inflammation: Evaluation by fluorodeoxyglucose positron emission tomography. J. Am. Coll. Cardiol. 2006,

32. Wu, Y.W.; Kao, H.L.; Huang, C.L.; Chen, M.F.; Lin, L.Y.; Wang, of 3-month atorvastatin therapy on arterial infla calcification, abdominal adipose tissue and circulating biomarkers. Eur. J. Nucl. Med. Mol. Imaging 2012, 39, 399307.

3. Eveborn, G.W.; Schirmer, H.; Heggelund, G.; Lunde, P.; Rasmussen, K. The evolving epidemiology of valvular ao
stenosis. The Tromso study. Heart 2013, $99,396-400$.

34. Baumgartner, H.; Hung, J.; Bermejo, J.; Chambers, J.B. Evangelista, A.; Griffin, B.P.; lung, B.; Otto, C.M.; Pellikka, P.A.; Quinones, M.; American Society of Echocardiography;
European Association of Echocardiography. EchocardioEuropean Association of Echocardiography. Echocardio-
graphic assessment of valve stenosis: EAE/ASE recommendations for clinical practice I. Am. Soc. Echocardiogr 2009, 45 22, 1-23.

35. Task Force Members; Vahanian, A.; Alfieri, O.; Andreotti, F Antunes, M.J.; Baron-Esquivias, G.; Baumgartner, H. Borger, M.A.; Carrel, T.P.; De Bonis, M.; et al. Guidelines on the management of valvular heart disease (version 2012): Disease of the European Society of Cardiology (ESC) and the European Association for Cardio-Thoracic Surgery (EACTS). Eur. Heart J. 2012, 33, 2451-2496,

36. Lancellott, P.; Moura, L.; scu, B.A.; Tribouilloy, C.; Hagendorff, A.; Monin, J.L.; cardiography European Association of Echocardiogaphy recommendations for the assessment of valvular regurgtation. Part 2: Mitral and tricuspid regurgitation (native valve disease). Eur. J. Echocardiogr. 2010, 11, 307-332.

37. Lang, R.M.; Bierig, M.; Devereux, R.B.; Flachskampf, F.A.; Fos ter, E.; Pellikka, P.A.; Picard, M.H.; Roman, M.J.; Seward, Shanewise, J.S.; et al. Recommendations for chamber quantiraphy's Guidelines and Standards Committee and the Chamber Quantification Writing Growp with the European Association of Echocardiography, a branch of the European Society of Cardiology. J. Am. Soc. Echocard-

ogr. 2005, 18, 1440-1463.
38. Schiller, N.B.; Shah, P.M.; Crawford, M.; DeMaria, A.; Sahn D. Schnittger I. et al Recommendations for cuantita tion of the left ventricle by two-dimensional echocardiography. American Society of Echocardiography Committee on Standards, Subcommittee on Quantitation of Two-Dimensional Echocardograms. J. Am. Soc. Echocardiogr. 1989,

Nagueh,

Oh, J.K. S.Fiseth, Apton, C.P.; Gillebert, T.C.; Marino, P.N. Pellikka, P.A.; Evangelisa, A. Recommendations for the evauation of left ventricular diastolic function by echocardiography. Eur. J. Echocardiogr. 2009, 10, 165-193.

40. Rudski, L.G.; Lai, W.W.; Afilalo, J.; Hua, L.; Handschumacher

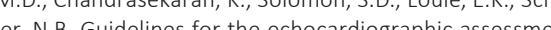

of the right heart in adults: A report from the American Society of Echocardiography endorsed by the European

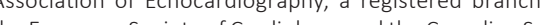
ty of Echocardiography. Am. Soc. Echocardiogr. 2010, $23,685-713$

MenaQ7. Avalable online. http://menaq7.com/whymenaq7/varieties/ (accessed on 2 March 2018).

Ahmed, N.: Vermeer, C. Beulens, IW. The effect E. menaquinone 7 supplementation on circulting species of matrix Gla protein. Atherosclerosis 2012, 225, 397-402.

Westenfeld, R.; Krueger, T.; Schlieper, G.; Cranenburs. Magdeleyns, E.J.; Heidenreich, S.; Holzmann, S.; Vermeer, K., Jahnen-Dechent, W.; Ketteler, M., et al. Effect of vitamin hemodialysis patients: A randomized Trial. An $J$ Kidy 2012, 59, 186-195.

S Ten Cate, H.; Shearer, M.J.; Vermeer, C.; Schurgers, L.J. eftect of low-dose supplements of menaquinone-7 (vitamin K2) on the stability of oral anticoagulant treatment Doser

Theuwissen, E.; Cranenburg, E.C.; Knapen, M.H.; Magdeleyns, E.J.; Teunissen, K.J.; Schurgers, L.J.; Smit, E.; Vermeer C. Low-dose menaquinone-7 supplementation improved bin generation in healthy subjects. Br. J. Nutr. 2012, 108

De, A.B.; Pedersen, T.R.; Boman, K.; Brudi, P.; Chambers, J.B.; Egstrup, K.; Gerdts, E.; Gohlke-Barwolf, C.
Holme, I.; Kesaniemi, Y.A.; et al. Intensive lipid lowerin with simvastatin and ezetimibe in aortic stenosis. N. Engl. J. Med. 2008, 359, 1343-1356

Chan, K.L.; Teo, K.; Dumesnil, J.G.; Ni, A.; Tam, J.; Investigators, A. Effect of Lipid lowering with rosuvastatin on proprogression observation: Measuring effects of ros suvastatis (ASTRONOMER) trial. Circulation 2010, 121, 306-314.

48. Van der Linde, D.; Yap, S.C., van Dijk, A.P.; Budts, W.; Pieper, P.G.; van der Burgh, P.H.; Mulder, B.J.; Witsenburg, M. Cuypers, J.A., Lindemans, J., et al. Effects of rosuvastatin on progression of stenosis in adult patients with congenita 265-271.

Fi. Francis, J.M. M.; Joseph, J.; Gerry, S.; Kar mitsos, T.D., Prendergast, B.D.; Banning, A.P.; Neubauer, S, Myerson, S.G. A prospective, double-blind, randomized controlled trial of the angiotensin-converting enzym Cardior Ramiprin Aortic Stenosis (RIAS thic

shaw, C. Richards, A.M. Edwards B. F. Whang, A.A.; Occledlander, D.; Williams, M.; et al. A randomized trial of the aldosterone-receptor antagonist eplerenone in asympto matic moderate-severe aortic stenosis. Am. Heart J. 2008, $156,348-355$

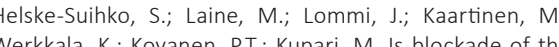
Renin-Angiotensin system able to reverse the structura and functional remodeling of the left ventricle in severe aortic stenosis? J. Cardiovasc. Pharmacol. 2015, 65, 233-240. 


\section{SUPPLEMENTAL MATERIAL}



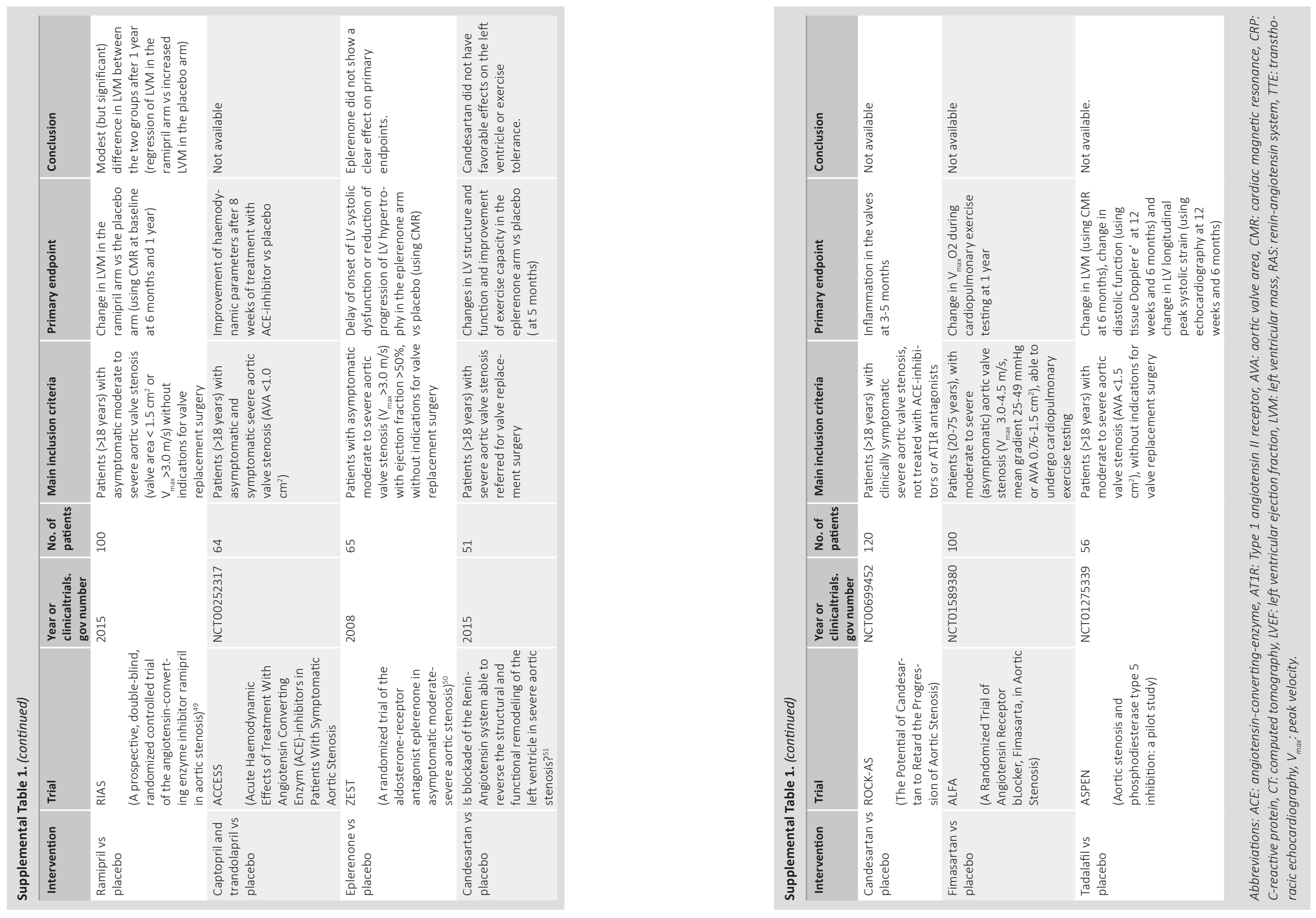

Chapter 5 


\section{Role of calcification in the progression of aortic valve stenosis: involvement of vitamin K-dependent Matrix Gla Protein}

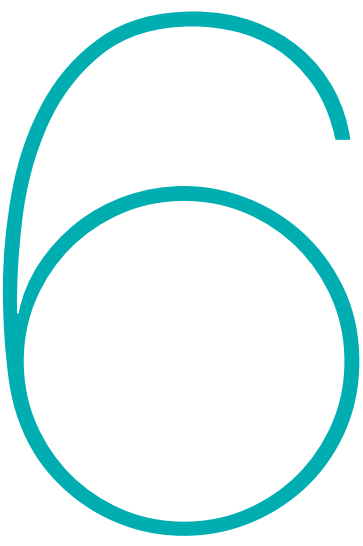

Frederique E.C.M. Peeters ${ }^{1}$, A.M.G. Jaminon², D. Peters ${ }^{1}$, M. Suverein ${ }^{3}$, J. Mesu', R. Lorusso ${ }^{4}$, P. Segers ${ }^{4}$, S. Kats ${ }^{4}$, H.J.G.M. Crijns ${ }^{1}$, S.J.R. Meex ${ }^{5}$, B.L.J.H. Kietselaer ${ }^{6}$, L.J. Schurgers

${ }^{1}$ Maastricht University Medical Center+ and CARIM, department of Cardiology, Maastricht, the Netherlands,

${ }^{2}$ Maastricht University and CARIM, department of Biochemistry, Maastricht, the Netherlands,

${ }^{3}$ Maastricht University Medical Center+ and CARIM, Intensive Care, Maastricht, the Netherlands,

${ }^{4}$ Maastricht University Medical Center+ and CARIM, department of Cardiothoracic Surgery,

${ }^{5}$ Maastricht University Medical Center+ and CARIM, department of Clinical Chemistry, Maastricht, the Netherlands,

${ }^{6}$ Zuyderland Medical Centre, Department of Cardiology, Heerlen, the Netherlands 


\section{ABSTRACT}

Background: Differentiation of valvular interstitial cells (VICs) to an osteoblast-like phenotype is thought to play a central role in progression of aortic valve stenosis (AS). Matrix Gla Protein (MGP) is a potent calcification inhibitor, and its expression was found to be reduced in diseased VICs, contributing to valvular calcification. Given the persisting need to intervene in the progression of AS, this study aimed to clarify the role of MGP in AS, by histopathologically mapping MGP distribution.

Methods: Aortic valves of 50 patients undergoing aortic valve replacement were collected and processed for analysis. Calcification volume of the valvular leaflets was determined ex vivo using micro-computed tomography. (Immuno)-histological staining with Von Kossa, osteocalcin (OC) and uncarboxylated MGP (ucMGP) was performed in a total of 40 valves.

Results: uCMGP was observed in areas extending from borders of regions showing significant calcification and in areas without significant calcification, suggesting involvement of MGP in early phases of inhibition of calcification. On the contrary, Von Kossa and osteocalcin positivity were specifically found within developed calcified areas. At last, calcification volume correlated well with the calcification quantification found in the leaflet tissue.

Conclusion: Our data provide evidence for a role of vitamin K-dependent proteins in AS. UCMGP is present in areas preceding calcification and also in regions with macro-calcification. With that our data supports further investigation of MGP as a potential follow-up strategy and druggable targets in AS.
INTRODUCTION

Aortic valve stenosis (AS) is commonly encountered in clinical practice in cardiology and its prevalence and health care burden will increase with an increasing elderly population. ${ }^{1}$ Once present, AS is a progressive disease. Untreated severe AS will lead to heart failure, cardiac arrhythmia and sudden cardiac death. A large variety of trials investigated potential pharmacological therapies, but at present none has proven to be irrefutable effective to reduce or reverse clinical progression of the disease. ${ }^{2,3}$ Therefore, aortic valve replacement (AVR) or transcatheter aortic valve implantation (TAVI) remain the sole effective treatment options for (symptomatic) severe AS at present. ${ }^{4}$

Inflammation, fibrosis and calcification are involved in AS, each having a phase of predominance in the initiation or progression. The exact mechanisms and the extent to which these drive AS progression are still under debate. Whereas inflammation is considered as the most dominant feature in the initiation phase of disease, fibrosis and primarily calcification predominate durin progression of AS, ultimately resulting in severe valvular stiffening and narrowing. Differentiation of valvular interstitial cells (VICs) from a myofibroblast phenotype in an osteoblast-like phenotype is thought to play a central role, driven by multiple regulatory pathways including bone morphogenetic proteins (BMPs). ${ }^{2,3,5-7}$ Within the BMP-family, BMP-2 is a key protein in the phenotypic switching of VICs and with that, in valvular calcification. ${ }^{8}$ Physiologically, BMP is inhibited by Matrix Gla Protein (MGP), a well-known inhibitor of vascular calcification. ${ }^{9,10}$ MGP is a vitamin $\mathrm{K}$-dependent protein, in need of carboxylation to exert its function (inactive MGP: uncarboxylated, (ucMGP); active, carboxylated MGP (cMGP)) ${ }^{10-12}$ Recent studies show that a decreased expression or activity of MGP in isolated VICs increases progression of valvular calcification. ${ }^{13,14}$ Besides forming a complex with BMP-2, hence inhibiting the BMP-2 pro-mineralizing activity, MGP exerts its function via a second mechanism by directly binding to hydroxyapatite and inhibiting growth of crystals. ${ }^{15}$

There is a persisting need to intervene in the progression of AS, and thus it is important to find targets to intervene in the processes of calcification. Therefore, this study aimed to histopathologically map markers of calcification involved in AS and more specifically Matrix Gla Protein, and to explore to what extent they are active in patients with established AS. Additionally, we analyzed how calcification visualized by micro-computed tomography (micro-CT) correlates with these results.

\section{METHODS}

\section{Patient population and tissue collection}

In this prospective, cross-sectional observational study, human aortic valves were obtained from 50 patients scheduled for (isolated or combined) aortic valve replacement at Maastricht University Medical Center+ (MUMC+). Clinical information was obtained from the electronic hospital charts and this study was approved by the local Institutional Review Board. All patients provided informed consent prior to final inclusion.

\section{Tissue preparation}

Valvular tissue samples were transported from the operating room in Paraformaldehyde $4 \%$ (PFA). Tissue samples were processed in different orientations (Figure 1) and embedded in paraffin $(n=40)$. Tissue samples were decalcified in $4 \mathrm{M} \mathrm{HCl}$ overnight. Tissue samples were sectioned in 5 um sections using a Microtome (Leica Reichert Jung 2035, Germany) and collected on glass slides. 


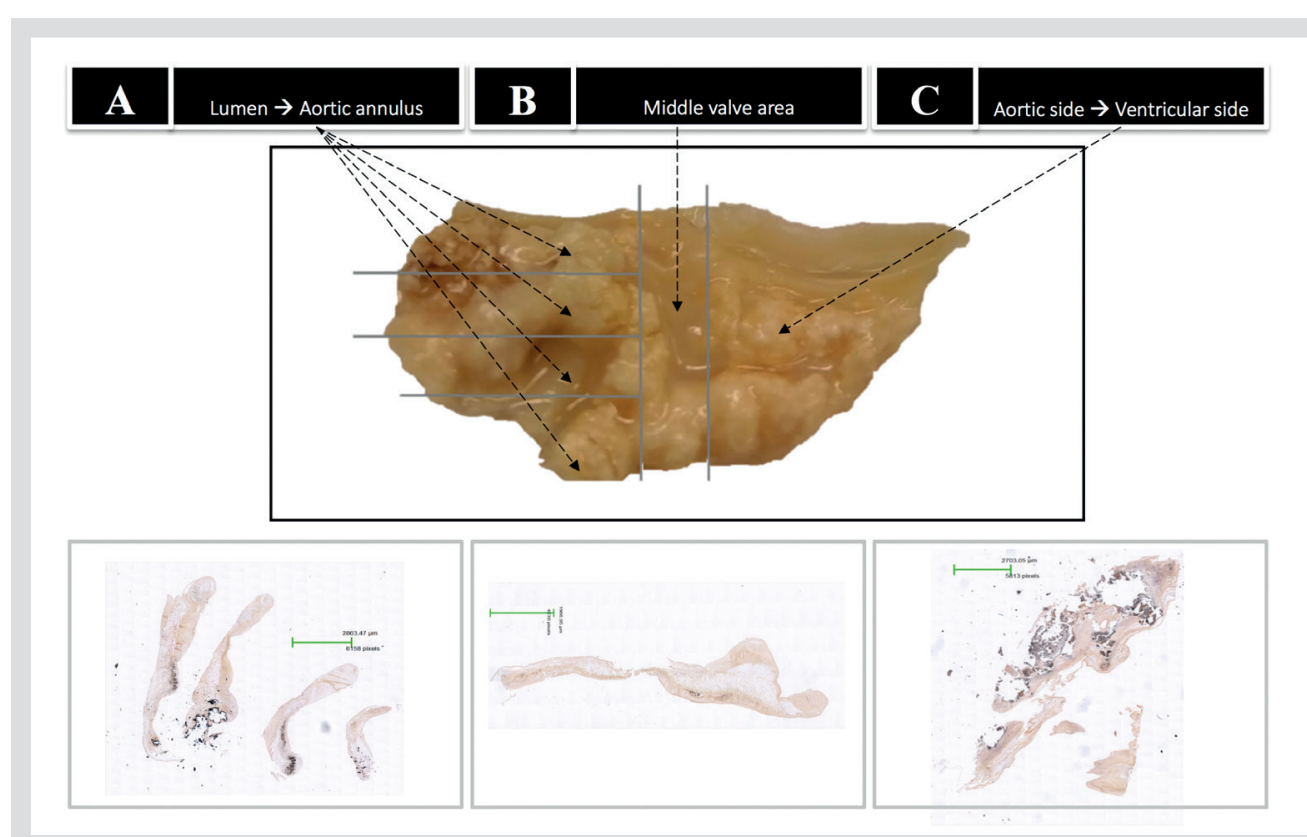

Figure 1. Orientation of valve leaflet slicing sections (viewed from aortic side). A: Slices from lumen to annulus, B: Slices from middle valve area, C: Slices from aortic to ventricular side.

(Immuno-) histochemical staining and analyses

Adjacent sections were stained using (immuno-) histochemistry techniques for markers for calcification von Kossa, osteocalcin (OC) and UCMGP. Prior to tissue analyses, whole tissue reference images were taken with the VENTANA iscan HT slide scanner (Ventana medical systems Inc, Roche). Valve leaflets were analyzed using two methods, described below.

Method I: overall calcification of valve leaflets

Quantitative analysis of calcification burden (von Kossa) in the valve leaflets was performed using an in-house programmed macro for image processing software FIJI (Fiji Is Just Image J, US National Institutes of Health, Bethesda, MD, USA). Staining intensities were converted to grey values and these grey values were quantified, resulting in a calcified area as percentage (\%) of the total valve

\section{Method II: region selection}

From tissue reference images, regions of heavy and light calcification were selected within valve leaflets in 10x magnifications using Ventana image viewer (Ventana Medical Systems Inc, Roche, Tucson, Arizona, USA). These regions were classified based on von Kossa positivity, scaled from to 5 , representing increasing calcification. Adjacent sections stained for UcMGP and osteocalcin were analyzed accordingly.

\section{Gross calcification quantification}

Ex vivo aortic valve calcification was quantified in 40 valves using a cone-beam computed tomography (CT) based image-guided irradiation unit (micro-IGRT; Precision X-ray/UHN, X-Rad 225CX) in
$40 \mathrm{kV}$ setting. All slides were scanned in 360 degrees in $512 \times 512$ pixel format. Total valvular volume (in $\mathrm{cm}^{3}$ ), calcification volume (using a $\geq 1500$ pixel threshold) and non-calcified volume (using $<1500$ pixel threshold) of each valve were calculated using OsiriX software (OsiriX Imaging Software, Geneva, Switzerland). The optimal threshold was determined using visual inspection of ex vivo valvular macrocalcification and corresponding areas of calcification on micro-CT images. Calcification volume was reported as percentage (\%) of the total valve leaflet volume.

\section{Statistical analyses}

Statistical analyses were performed using SPSS version 22 (IBM Corp, Armonk, NY).

Normally distributed continuous variables are expressed as mean \pm standard deviation (SD) and non-normally distributed continuous variables as median [interquartile range] (IOR). Pearson's Correlation Coefficient was used to determine correlations. Categorical and ordinal variables are expressed as absolute numbers and percentages and tested using the $\chi^{2}$-test and the Mantel-Haenszel test of trend.

\section{RESULTS}

\section{Population characteristics}

Mean ( $\pm S D$ ) age of the population was $66 \pm 9$ years, $22 \%$ were female and $40 \%$ of patients were known with a bicuspid aortic valve (BAV) prior to surgery. Of all patients, $48 \%$ underwent isolated aortic valve replacement for AS ( $2 \%$ AVR for aortic valve regurgitation) and $50 \%$ underwent AVR in combination with coronary artery bypass grafting (CABG) or ascending aortic repair/replacement. Population characteristics are presented in Supplemental Results Table 1.

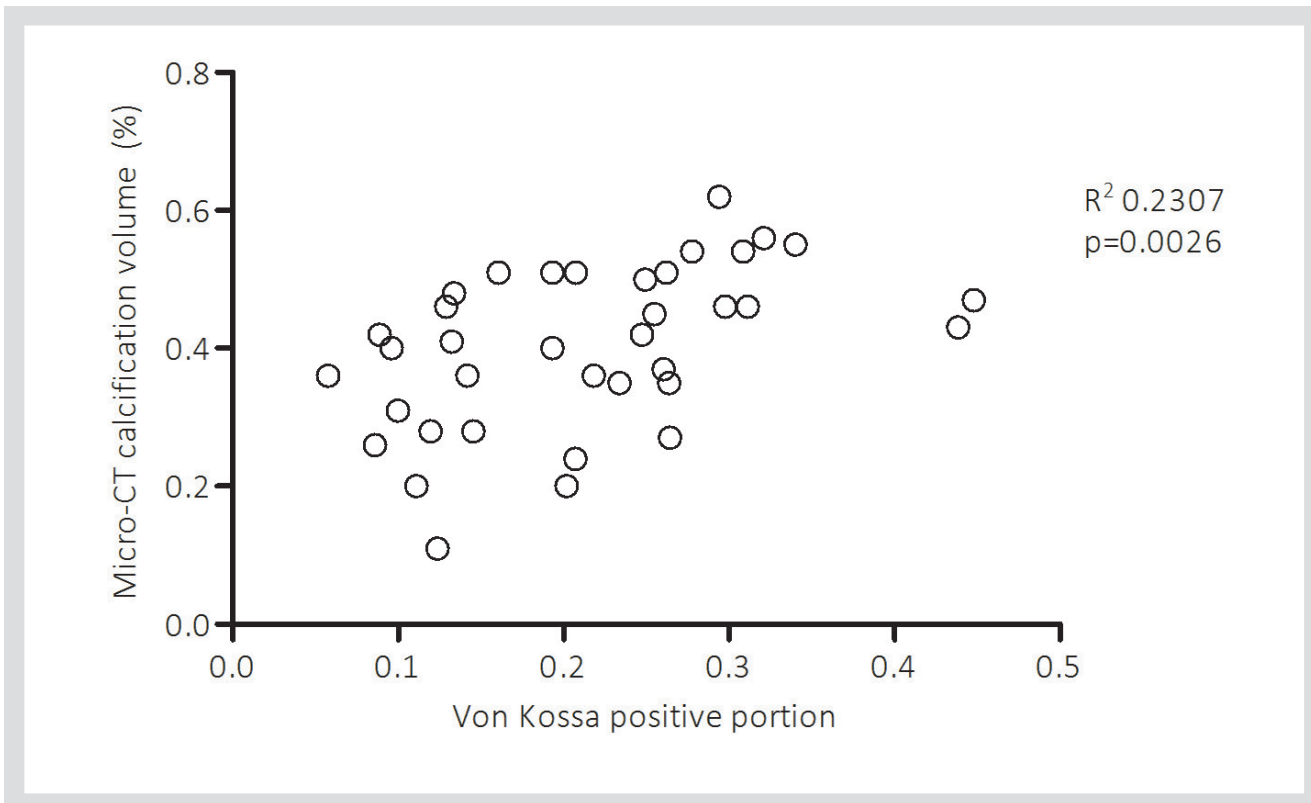

Figure 2. Pearson Correlation between Von Kossa staining and corresponding calcification volume on micro-CT (percentage, \%) 


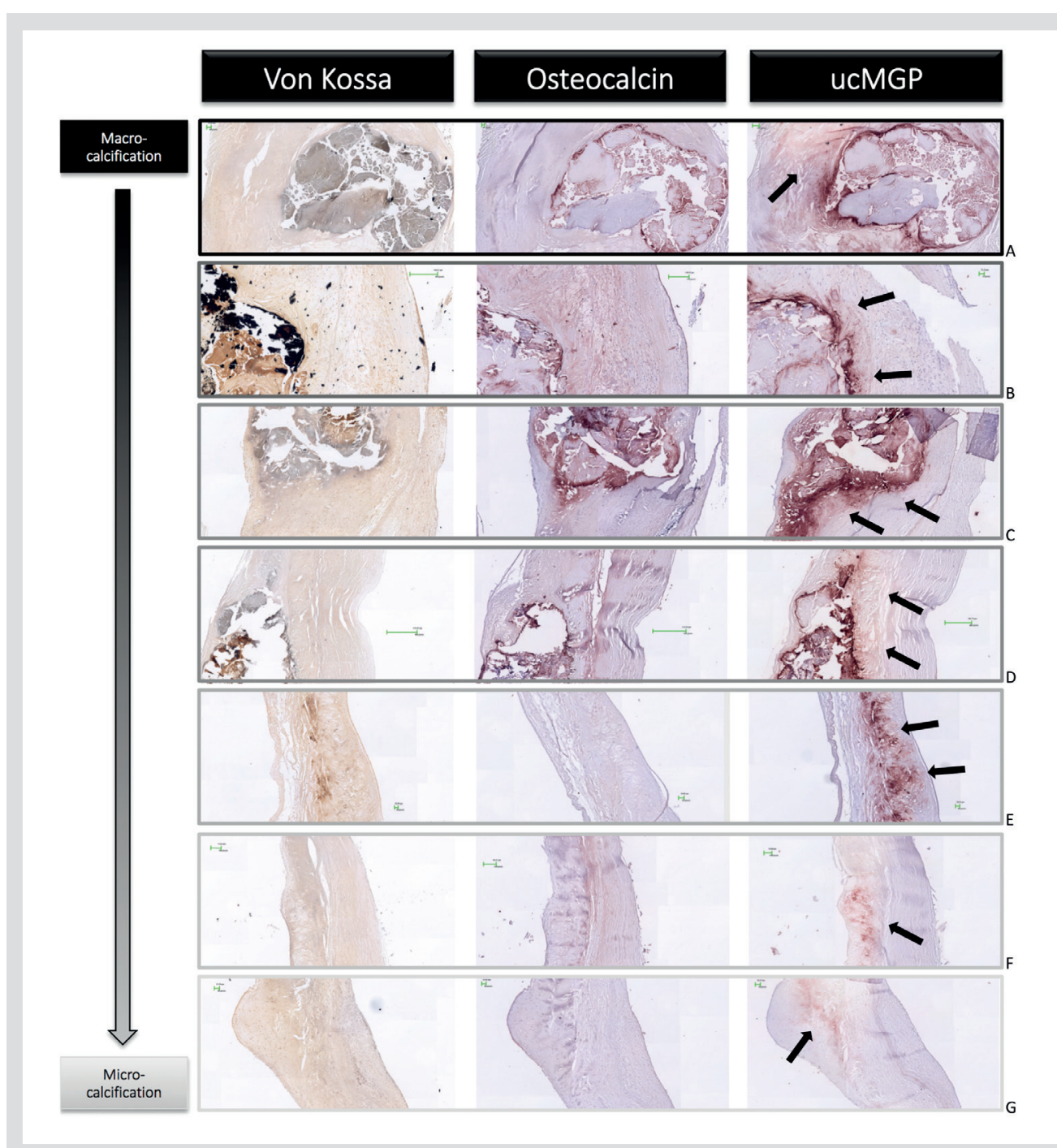

Figure 3. Representative images of calcified regions and regions with microcalcification. Representative images of von Kossa, osteocalcin and UCMGP (immuno-) histochemical staining of regions with macrocalcification (A-D) to early calcification and microcalcification (E-G). Black arrows indicate regions of uCMGP positivity

\section{Quantification of tissue calcification and calcification on micro-CT}

To quantify ex vivo calcification volume, aortic valve leaflets were scanned using a micro-CT scanner. and calcification volume and total valve volume were determined. Quantification of calcification in the valve leaflets was assessed using von Kossa staining according to method I. Indexed calcification volume to total leaflet volume (\%) was significantly correlated with von Kossa positivity (presented as the relative area of staining positivity), $p=0.0026\left(R^{2} 0.2307\right)$ (Figure 2).

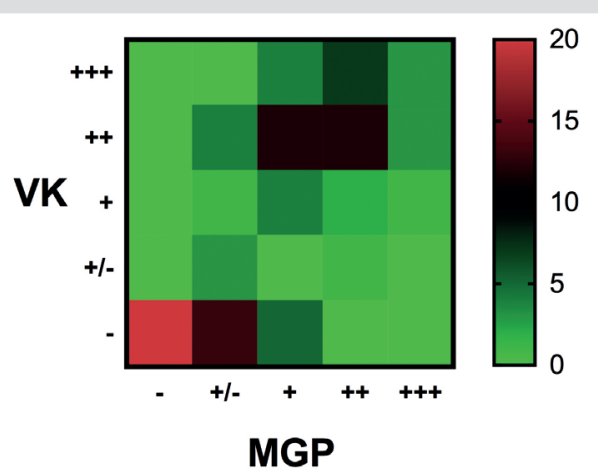

MGP
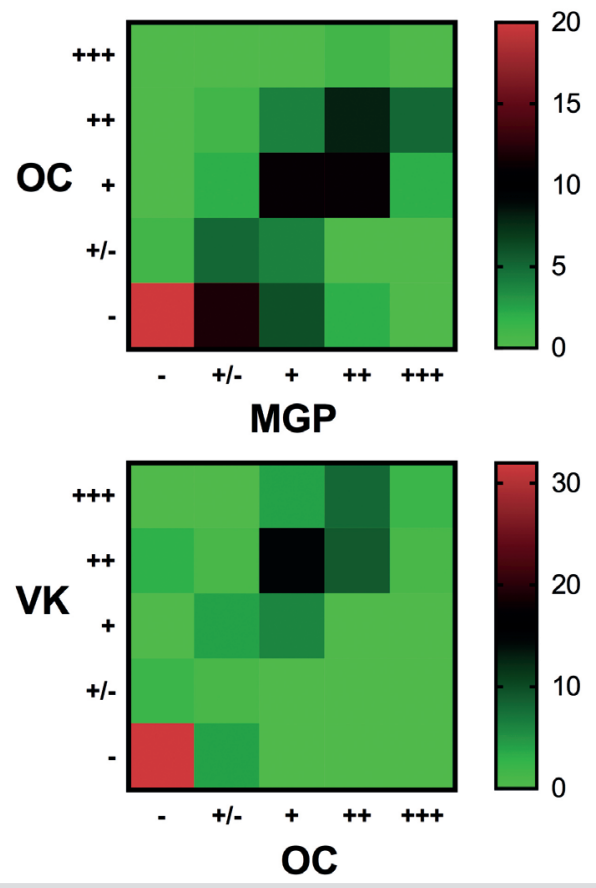

Figure 4. Correlation between Von Kossa, osteocalcin and ucMGP stainings. Upper panel: heatmap von Kossa (VK) and UCMGP (MGP). Middle panel: heatmap osteocalcin (OC) and uCMGP. Lower panel: heatmap von Kossa and osteocalcin. - , +/-. +, ++, +++ represent scoring of degree of positivity of staining (low[---high[+++]]. Color density represents number of counts of staining in valve regions

\section{Analysis of specific regions in valve leaflets: ucMGP surrounding regions of calcification,} representing microcalcification?

Specific regions were selected by visual assessment of whole sections on macro-calcification and Von Kossa positivity. In Figure 3, representative images of selected areas are shown including all three stainings. In regions with significant calcification (Figure 3, A-D), both Von Kossa and the osteochondrogenic OC staining were positive within the calcified region and at the borders of 
calcified areas. Moreover, they followed similar patterns in regional positivity. ucMGP staining showed positivity in the borders of calcification. However, in the surroundings of calcification regions, ucMGP showed a typical pattern of positivity, as can be appreciated in the upper panels of Figure 3 (black arrows). Also, in regions with low stain positivity of Von Kossa and osteocalcin (Figure 3, E-G), ucMGP positivity was found (black arrows). These findings suggest that the presence of UCMGP is involved in the initiation of microcalcification and macro-calcification in the later stages of AS.

After visual assessment, staining was classified (in 5 categories) according to their positivity in order to assess mutual associations. Figure 4 shows the results of these assessments. A MantelHaenszel test of trend was run to determine whether a linear association existed between the von Kossa and UCMGP, osteocalcin and UCMGP and von Kossa and osteocalcin respectively. The Mantel-Haenszel test of trend showed a statistically significant linear association between von Kossa and ucMGP $\left(\chi^{2}=55.429, p<.001, r=.768\right)$, osteocalcin and ucMGP $\left(\chi^{2}=52.024, p<.001, r=.744\right)$ and von Kossa and osteocalcin $\left(\chi^{2}=71.078, p<.001, r=.865\right)$ respectively.

\section{DISCUSSION}

In this study, we examined ucMGP patterns in valve tissue of patients with AS, in relation to tissue calcification and osteochondrogenic markers. We report three main findings: First, ucMGP was detected in areas extending from borders of regions showing heavy calcification, whereas von Kossa and osteocalcin positivity was typically observed within the calcified area. Upon quantification, both von Kossa and osteocalcin showed a positive association with ucMGP. Second ucMGP was found in valvular regions without presence of macrocalcification. In absence of calcification and osteochondrogenic markers in these regions, these findings suggest that ucMGP is present in regions contiguous with existing (macro)calcification and serves as an early marker of beginning processes of calcification, that is, with development of microcalcifications. These findings are based on cross-sectional stainings. Since longitudinal follow-up of these processes is of interest, application of specific imaging tools such as ${ }^{18} \mathrm{~F}$-sodium fluoride $\left({ }^{18} \mathrm{~F}-\mathrm{NaF}\right)$ could be valuable. ${ }^{18} \mathrm{~F}$-sodium fluoride $\left({ }^{18} \mathrm{~F}-\mathrm{NaF}\right)$ is a tracer used in Positron Emission Tomography (PET) for the detection of calcium metabolism. Clinically, it is used to detect bone metastasis in cancer, but it has also been employed in trials studying pharmacological intervention in $\mathrm{AS} \cdot{ }^{16}$ The application of ${ }^{18} \mathrm{~F}-\mathrm{NaF}$ in $\mathrm{PET} / \mathrm{CT}$ has been shown to identify active calcification in AS whilst other imaging modalities enable visualization of macro-calcification solely. ${ }^{17,18}$ This specific tracer binds to hydroxyapatite crystals in regions with developing calcification, and so does MGP.15,17,18 Thus, it would be of interest to see whether ${ }^{18} \mathrm{~F}-\mathrm{NaF}$ shows a similar distribution in our tissue samples to confirm the utility of a clinically available tool to visualize MGP patterns specifically.

Third, tissue calcification correlated with calcification volume found on micro-CT, serving as a tool to corroborate our findings in tissue. Although routine practice incorporates the Agatston score on CT instead of calcification volume to classify AS severity, calcification volume determined with micro-CT appeared to correlate with AS severity in other studies.,19

Studies investigating the role of MGP specifically in human aortic valve tissue are sparse. Two studies investigated the role of MGP in human VICs, and found a decreased expression anti-calcification protein in AS. With that, they provided further evidence for the involvement of MGP in the pathophysiology of AS. ${ }^{13,14}$ Our study adds information by studying the patterns of the inactive variant of MGP (ucMGP) in aortic valve tissue and serves as a foundation to further study the role of MGP in progression of AS. Moreover, ${ }^{18} \mathrm{~F}-\mathrm{NaF}$ PET/CT has been shown to identify active calcification in AS.
At last, circulating levels of UCMGP have been correlated with disease progression and cardiac function, providing evidence to support the potential importance of MGP in AS. ${ }^{20,21}$ Since the availability of active MGP can be influenced by vitamin $\mathrm{K}$ antagonists (VKA) and vitamin $\mathrm{K}$, it is a topic of interest to study.22,23 future studies will have to investigate its utility in follow-up and as a potential target for medical interventions.

\section{Study limitations, conclusion and future perspectives}

As with any cross-sectional study, this study could not establish a causal relation between ucMG and progression of calcification in AS. Moreover, most of our patients had moderate to severe As and high levels of calcification, and thus our results might not be applicable to the whole spectrum of aortic valve disease and more specifically to early phases of disease. Furthermore, we quantified calcification ex vivo using thresholds in micro-CT images, due to impossibility of using the standardized Agatston score quantification in the ex vivo samples. It should therefore be considered as a gross measure, in need for further refinement and standardizing. However, taking the abovementioned limitations into account, to the best of our knowledge, we provide first evidence of MGP distribution patterns in valvular tissue and with that, our data supports further investigation of MGP as a potential follow-up strategy and target in AS. Future analyses of the current study will include other markers at tissue level (f.i. fibrosis, inflammation) and circulating biomarkers. 


\section{REFERENCES}

disty: disease prevalence and number of candidates and modeling study. Journal of the American College of Cardiology 2013;62:1002-12.

2. Pawade TA, Newby DE, Dweck MR. Calcification in Aortic

et al. Calcific aortic valve stenoin the heart: A biomolecular approach towards diagnosis and treatment. European heart journal 2017.

4. Baumgartner H, Falk V, Bax JJ et al. 2017 ESC/EACTS Guidelines for the management of valvular heart disease. Euro-

pean heart journal 2017,38.2739-2791.

calcific aortic value disease. Circulation 2012:125:9-11.

Hutcheson ID, Aikawa E, Merryman WD. Potential drug targets for calcific aortic valve disease. Nature reviews Cardiology 2014;11:218-31.

. Mohler ER, 3rd, Gannon F, Reynolds C, Zimmerman R. cardiac valves. Circulation 2001:103:1522-8.

8. Gomez-Stallons MV, Wirrig-Schwendeman EE, Hassel KR, Conway SJ, Yutzey KE. Bone Morphogenetic Protein Signaing Is Required for Aortic Valve Calcification. Arteriosclersis, thrombosis, and vascular biology 2016;36:1398-405. Luo G, Ducy $P$, Mckee MD et al. Spontaneous calcification of arteries and cartlage in r

K-dependent carboxylation of matrix Gla $\mathrm{CP}$. Vitamin switch to control ectopic mineralization. Trends in molecular medicine 2013;19:217-26.

1. Schurgers LJ, Cranenburg EC, Vermeer C. Matrix Gla-pro-

12. Schurgers $L$, Spronk HM, Skepper JN et al. Post-transla tional modifications regulate matrix Gla protein function importance for inhibition of vascular smooth muscle ce calcification. Journal of thrombosis and haemostasis : JTH

13. Chiyoya M, Seya K, Yu Z et al. Matrix Gla protein negatively regulates calcification of human aortic valve interstitia
cells isolated from calcified aortic valves. Journal of pharmacological sciences 2018;136:257-265.

14. Venardos N, Bennett D, Weyant MJ, Reece TB, Meng X, Fullerton DA. Matrix Gla protein regulates calcification of the aortic valve. The Journa of surgical tesearch 2015,199:1-6. ectopic calcification by a direct interaction with hydroxyapatite crystals. Journal of the American Chemic Society 2011;133:18406-12.

16. Peeters F, van Mourik MJW, Meex SJR et al. Bicuspid Aortic Valve Stenosis and the Effect of Vitamin K2 on Calcificatio Using (18)F-Sodium Fluoride POsitron Emission TomograDesign. Nutrients 2018;10.

17. Dweck MR, Jenkins WS, Vesey AT et al. 18F-sodium fluoride uptake is a marker of active calcification and disease progression in patients with aortic
vascular imaging 2014;7:371-8

18. Irkle A, Vesey AT, Lewis DY et al. Identifying active vascular sion tomography Nature communications 2015:6:7495.

Chitsaz S, Gundiah N. Blackshear C et al. Correlation of calexcised aortic valves by micro-co 作 (12;21:320-7.

. Capoulade R, Cote N, Mathieu P et al. Circulating levels of matrix gla protein and progression of aortic stenosis: a subMeasuring Effects of rosuvastatin (ASTRONOMER) triat The Canadian journal of cardiology 2014:30:1088-95.

Ueland T, Gullestad L, Dah CP et al. Undercarboxylated matrix Gla protein is associated with indices of heart failure and mortality in symptomatic aortic stenosis. Journal of Brend medicine 2010;268.483,92.

Slower Promentation: Results From a Prospective Intervention Proof-of-Concept Study. Circulation 2017:135:2081-2083. Koos R, Mahnken AH, Muhlenbruch G et al. Relation of ora anticoagulation to cardiac valvular and coronary calcium assessed by mulisile spiral compused tomography. The 


\section{SUPPLEMENTAL METHODS}

\section{Von Kossa staining}

Paraffin embedded tissue sections were deparaffinized and rehydrated to demi water. Slides were incubated in $1 \% \mathrm{AgNO}$ for 5 minutes and washed in running demi water for 5 minutes. Sections were incubated with sodaformol (see Supplemental Methods table 1 for formulation) for 1 minute and washed in running demi for 5 minutes. Slides were incubated in a $5 \% \mathrm{Na}_{2} \mathrm{~S}_{2} \mathrm{O}_{3}{ }_{3} \mathrm{H}_{2} \mathrm{O}$ solution for 5 minutes and washed in running demi water for 5 minutes. Tissue sections were counterstained with Nuclear Fast Red (642420, Klinipath, ready to use) for 1 minute and washed in running demi for 5 minutes. Slides were dehydrated to xylol and were mounted with entallan (6383111-1, VWR, USA).

\begin{tabular}{l|l|}
\hline \multicolumn{2}{l}{ Supplemental Methods Table 1. Sodaformol formulation } \\
\hline Volume & Component \\
\hline $35 \mathrm{ml}$ & Millia \\
$40 \mathrm{ml}$ & $12.5 \% \mathrm{NaCO}_{3}$ \\
$25 \mathrm{ml}$ & $37 \%$ Formaldehyde \\
\hline
\end{tabular}

\section{SUPPLEMENTAL MATERIAL}

\section{Immunohistochemical osteocalcin staining}

Paraffin embedded tissue sections were deparaffinized and rehydrated to demi water. After ethanol endogenous peroxidase was blocked by $0.3 \% \mathrm{H}_{2} \mathrm{O}_{2}$ in methanol solution ( $\mathrm{H}-0904$ Sigma-Aldrich, USA) for $30 \mathrm{~min}$. Tissue sections were placed in $90^{\circ} \mathrm{C}$ target retrieval solution (see Supplemental Methods Table 2 for formulation) and left to cool down for 40 min. Sections were washed thrice in Tris buffered saline ( $\mathrm{pH} 7.4$ )(TBS). Blocking was performed with 5\% goat serum in TBT (see Supplemental Methods Table 3 for formulation) for 1 hour. Rabbit anti-OsteoCalcin (sc-30044, Santa Cruz, USA) 1:500 in TBT was incubated to the tissue sections overnight at $4^{\circ} \mathrm{C}$. Slides were washed trice with TBS and goat anti-rabbit poly-HRP (Immunologic, DPVR110HRP, ready-to-use) was incubated for $60 \mathrm{~min}$ at room temperature (RT). The tissue sections were washed twice in TBS before they were stained with Novared (SK-4800, Vector Laboratories, UK) for 3 minutes. Excess staining solution was washed off in demineralized (Demi) water for 5 minutes and tissue sections were counterstained in hematoxylin (ghs-2-32, Sigma-Aldrich, USA) for $1 \mathrm{~min}$ Slides were placed under running tap water to develop color for $10 \mathrm{~min}$. Tissue sections were mounted in aqueous mounting medium Imsol (4058, Klinipath, The Netherlands) (1:5) and afterwards covered in Entellan (Merck, Germany).

Supplemental Methods Table 2. Target retrieval solution formulation

\begin{tabular}{|l|l|}
\hline Concentration & Component \\
\hline $10 \mathrm{mM}$ & Tri-Na-citrate \\
\hline pH 6 & Citric acid \\
\hline
\end{tabular}




\begin{tabular}{|l|l|}
\hline Concentration & Component \\
\hline $1 \%$ & BSA \\
\hline $0.1 \%$ & Tween 20 \\
\hline
\end{tabular}

\section{SUPPLEMENTAL RESULTS}

Supplemental Results Table 1. Population characteristics

\begin{tabular}{|c|c|}
\hline \multicolumn{2}{|c|}{ Supplemental Results Table 1. Population characteristics } \\
\hline & Population \\
\hline \multicolumn{2}{|l|}{ Demographics } \\
\hline Age (years) & $66 \pm 9$ \\
\hline Female sex & $11(22)$ \\
\hline BMI $\left(\mathrm{kg} / \mathrm{m}^{2}\right)$ & $28.2 \pm 3.7$ \\
\hline \multicolumn{2}{|l|}{ Patient history } \\
\hline Hypertension & $29(58)$ \\
\hline Coronary artery disease & $24(48)$ \\
\hline Myocardial infarction & $7(14)$ \\
\hline Diabetes Mellitus & $9(18)$ \\
\hline Hypercholesterolemia & $15(30)$ \\
\hline Current smoker & $11(22)$ \\
\hline \multicolumn{2}{|l|}{ Medication } \\
\hline ACE inhibitor & $10(20)$ \\
\hline Angiotensin receptor blocker & $13(26)$ \\
\hline$\beta$-blocker & $24(48)$ \\
\hline Diuretics & $15(30)$ \\
\hline Statins & $30(60)$ \\
\hline \multicolumn{2}{|l|}{ Echocardiography } \\
\hline Severe aortic valve stenosis & $40(80)$ \\
\hline Maximum pressure gradient $(\mathrm{mmHg})$ & $74 \pm 22$ \\
\hline Mean Pressure gradient $(\mathrm{mmHg})$ & $43 \pm 14$ \\
\hline Doppler Index (VR) & $0.23 \pm 0.06$ \\
\hline Aortic valve area $\left(\mathrm{cm}^{2}\right)$ & $0.9 \pm 0.2$ \\
\hline Left ventricular ejection fraction (\%) & $58 \pm 9$ \\
\hline \multicolumn{2}{|l|}{ Lab } \\
\hline Total cholesterol (mmol/L) & $4.79 \pm 1.22$ \\
\hline Low-density lipoprotein (mmol/L) & $2.66 \pm 1.11$ \\
\hline High-density lipoprotein (mmol/L) & $1.39 \pm 0.46$ \\
\hline Triglycerides (mmol/L) & $1.73 \pm 0.90$ \\
\hline Calcium (mmol/L) & $2.39 \pm 0.11$ \\
\hline Phosphate (mmol/L) & $1.01 \pm 0.18$ \\
\hline Alkaline Phosphatase (U/L) & $81.94 \pm 23.31$ \\
\hline
\end{tabular}

\section{Immunohistochemical uncarboxylated MGP staining}

Paraffin embedded tissue sections were deparaffinized and rehydrated to demi water. After ethanol endogenous peroxidase was blocked by $0.3 \% \mathrm{H}_{2} \mathrm{O}_{2}$ in methanol solution $(\mathrm{H}-0904$, Sigma-Aldrich, USA) for $30 \mathrm{~min}$. Tissue sections were placed in $90^{\circ} \mathrm{C}$ target retrieval solution (see Supplemental Methods Table 2 for formulation) and left to cool down for $40 \mathrm{~min}$. Sections were washed thrice in Tris buffered saline (pH 7.4)(TBS). Blocking was performed with $5 \%$ goat serum in TBT (see Supplemental Methods Table 3 for formulation) for 1 hour. Mouse anti ucMGP (Ma35-49GLU-MGP; B11A) 1:400 in TBT was added to the tissue and left overnight at $4^{\circ} \mathrm{C}$.

Slides were washed trice with TBS and goat anti-mouse HRP (DAKO, p0447) 1:100 was incubated for $60 \mathrm{~min}$ at room temperature (RT). The tissue sections were washed twice in TBS before they were stained with Novared (SK-4800, Vector Laboratories, UK) for 2 minutes. Excess staining solution was washed off in demineralized (Demi) water for 5 minutes and tissue sections were counterstained in hematoxylin (ghs-2-32, Sigma-Aldrich, USA) for 1 minute. Slides were placed under running tap water to develop color for $10 \mathrm{~min}$. Tissue sections were mounted in aqueous mounting medium Imsol (4058, Klinipath, The Netherlands) (1:5) and afterwards covered in Entellan (Merck, Germany) 
Sex-related differences in valvular fibrosis and calcification in aortic stenosis: application of a non-invasive imaging strategy using ${ }^{18} \mathrm{~F}-\mathrm{NaF} \mathrm{PET} / \mathrm{CT}$

Frederique E.C.M. Peeters, $\mathrm{MD}^{1}$, Mhairi K. Doris, MD², Timothy R.G. Cartlidge, $\mathrm{MD}^{2}$, Jacek Kwiecinski2 ${ }^{2}$ Tania A. Pawade'2, William S.A. Jenkins², Dorien M. Kimenai, Msc ${ }^{3}$, Steven J.R. Meex, PhD ${ }^{3}$, Bas L.J.H.Kietselaer, MD, PhD ${ }^{4}$, Harry J.G.M. Crijns, MD, $\mathrm{PhD}^{1}$, Marc R. Dweck, MD, PhD ${ }^{2}$

${ }^{1}$ Maastricht University Medical Centert, department of Cardiology and CARIM, P. Debyelaan 25, 6229 HX Maastricht, the Netherlands,

${ }^{2}$ Centre for Cardiovascular Science, University of Edinburgh, 47 Little France Crescent, Edinburgh EH16 4TJ, United Kingdom,

${ }^{3}$ Maastricht University Medical Center+, department of Clinical Chemistry and CARIM,

P. Debyelaan 25, 6229 HX Maastricht, the Netherlands,

${ }^{4}$ Zuyderland Medical Center, department of Cardiology Sittard/Heerlen, Henri Dunantstraat 5, 6419 PC Heerlen, the Netherlands 


\section{ABSTRACT}

Background: Aortic valve calcification (AVC) is a key factor in development and progression of aortic valve stenosis (AS). However, females tend to have less AVC for similar AS hemodynamic severity when compared to males. Recently, a dominant role for valvular fibrosis in females was suggested by histology.

Objective: To assess sex differences in AVC activity and fibrosis in AS using a clinically available combined ${ }^{18} \mathrm{~F}$-sodiumfluoride ( $\left.{ }^{18} \mathrm{~F}-\mathrm{NaF}\right)$ PET/CT.

Methods: One hundred and forty six patients (29 female, 117 male) with mild to severe aortic valve stenosis underwent combined ${ }^{18} \mathrm{~F}-\mathrm{NaF}$ PET and contrast-enhanced CT scanning. ${ }^{18} \mathrm{~F}-\mathrm{NaF}$ quantification was performed using the most diseased segment approach with maximum and mean target-to-background both calculated. The calcification:fibrosis ratio was calculated using calcification and fibrosis volumes on contrast-enhanced CT.

Results: Results regarding calcification activity were conflicting: male sex was associated with higher $\mathrm{TBR}_{\text {max' }}$ but $T B R_{\text {mean }}$ showed no significant differences. Significant differences between females and males existed in the calcification:fibrosis ratio on CT. Females showed a more dominant fibrosis pattern in relation to calcification, whilst males showed the opposite pattern (ratio $>1$ in $53 \%$ of males and $27 \%$ of females, $p=0.018$ ).

Conclusion: Males and females with aortic valve stenosis show a difference in dominant phenotype (fibrotic vs calcified). Female gender is associated with a predominantly fibrotic phenotype, whereas male gender is associated with a predominance of calcification. However, further work is required to clarify whether sex-differences in calcification activity exist.

Key words: aortic valve stenosis, calcification, calcification:fibrosis ratio, ${ }^{18} \mathrm{~F}-\mathrm{NaF} \mathrm{PET} / \mathrm{CT}$, imaging, sex 
described by Pawade et al. ${ }^{15}$ In short: for each PET/CT scan, registration in the axial, coronal and sagittal planes was performed between PET and CT angiography data in diastolic phase. The maximum ${ }^{18} \mathrm{~F}-\mathrm{NaF}$ uptake in the aortic valve was calculated by construction of polygon-shaped ROIs around the perimeter of the aortic valve in all slices comprising the valve after reorientation in the aortic valve plane. Mean and maximum standard uptake values (SUV $V_{\text {mean }}$ and SUV $V_{\text {max }}$ ) were generated for each slice. Subsequently, two contiguous segments displaying the highest SUV values were averaged to generate $S U V_{\text {mean }}$ and $S U V_{\text {max }}$ of the most diseased segment (MDS).

Blood-pool activity using mean SUVs was determined by averaging two ROIs $\left(2 \mathrm{~cm}^{2}\right.$ each) in the right atrium in two consecutive slices (at the level of the origin of the right coronary artery and one slice superiorly) Interobserver reproducibility was assessed in 15 cases by quantification of uptake values on the scans by two experienced operators (F.P. and M.D.), blinded for the results.

Target-to-background ratios (TBR) were computed. $T_{B R} R_{\max }$ was defined as the maximum uptake measured in the aortic valve divided by the mean SUV of the blood-pool. TBR mean $_{\text {mas }}$ defined as the mean uptake measured in the aortic valve divided by the mean SUV of the blood-pool.

Fibrosis volume and calcium volume were calculated using thresholds calibrated against the blood pool contrast load according to the methods described by Cartlidge et al (manuscript in preparation). Calcification volume was divided by fibrosis volume to obtain valvular calcification:fibrosis ratio.

\section{Statistical analyses}

Normally distributed continuous population characteristics are reported as mean \pm standard deviation (SD) and non-normal distributed continuous population characteristics as median and interquartile range [IQR]. Categorical data are reported as number $(n)$ and percentage $(\%)$. Linear regression analyses were used to evaluate associations between sex and valve TBR TBR and the valve calcification:fibrosis ratio. The outcome variables, TBR , TBR and calcification:fibrosis ratio were logarithmically transformed. The independent variable sex was examined in three models: unadjusted (model 1); model $1+$ aortic valve stenosis severity (peak aortic jet velocity) and LVOT diameter (model 2); and model $2+$ age, body surface area (BSA), presence of coronary artery disease, diabetes mellitus, hypertension, hyperlipidaemia and smoking (model 3). Differences in calcification:fibrosis ratio $>1$ and $<1$ were analyzed using the Mann-Whitney U-test. Interobserver variability testing was performed using the interclass correlation coefficient (ICC) and Bland-Altman plots. Overall, a p-value $<0.05$ was considered statistically significant. All statistical analyses were performed using SPSS statistics version 22 (IBM Corp, Armonk, NY).

\section{RESULTS}

\section{Population characteristics}

For the analysis of the present study, a total of 146 patients were analyzed: 29 females and 117 males. All patients had aortic stenosis at the time of scanning. In the population as a whole $25 \%$ $(n=37)$ had mild, 56\% ( $n=81)$ moderate and 16\% ( $n=23)$ severe AS. In females, $28 \%(n=8)$ had mild, $59 \%(n=17)$ moderate and $4 \%(n=1)$ severe AS. In males, 25\% $(n=29)$ had mild, 55\% $(n=64)$ moderate and $18 \%(n=22)$ severe AS. Population characteristics, including female/male characteristics are shown in table 1.
Table 1. Population characteristics

\begin{tabular}{|c|c|c|c|c|}
\hline & $\begin{array}{l}\text { Total population } \\
(n=146)\end{array}$ & $\begin{array}{l}\text { Female } \\
(n=29)\end{array}$ & $\begin{array}{l}\text { Male } \\
(n=117)\end{array}$ & P-value \\
\hline \multicolumn{5}{|l|}{ Demographics \& vital signs } \\
\hline Age (years) & $72 \pm 8$ & $72 \pm 7$ & $72 \pm 8$ & 0.667 \\
\hline $\mathrm{BSA}\left(\mathrm{m}^{2}\right)$ & $1.99 \pm 0.22$ & $1.78 \pm 0.17$ & $2.03 \pm 0.20$ & $<0.001$ \\
\hline Systolic bloodpressure (mmHg) & $149 \pm 20$ & $150 \pm 18$ & $148 \pm 21$ & 0.719 \\
\hline Diastolic bloodpressure (mmHg) & $78 \pm 12$ & $78 \pm 10$ & $77 \pm 12$ & 0.877 \\
\hline Heart rate (bpm) & $68 \pm 13$ & $68 \pm 13$ & $68 \pm 13$ & 0.899 \\
\hline \multicolumn{5}{|l|}{ Relevant medical history } \\
\hline Hypertension & $106(60.6)$ & $22(75.9)$ & $84(71.8)$ & 0.660 \\
\hline Coronary artery disease & $56(32.0)$ & $11(37.9)$ & $45(38.5)$ & 0.958 \\
\hline Smoking & $51(29.1)$ & $10(34.5)$ & $41(35.0)$ & 0.955 \\
\hline Diabetes mellitus & $34(19.4)$ & $6(20.7)$ & $28(23.9)$ & 0.712 \\
\hline Hyperlipidaemia & $82(46.9)$ & $18(62.1)$ & $64(54.7)$ & 0.474 \\
\hline \multicolumn{5}{|l|}{ Concomitant medications } \\
\hline Statin & $101(57.7)$ & $17(58.6)$ & $84(71.8)$ & 0.169 \\
\hline ACE inhibitor & $53(30.3)$ & $10(34.5)$ & $43(36.8)$ & 0.796 \\
\hline ARB & $29(16.6)$ & $7(24.1)$ & $22(18.8)$ & 0.519 \\
\hline Beta blocker & $57(32.6)$ & $10(34.5)$ & $47(40.2)$ & 0.574 \\
\hline Antiplatelet therapy & $78(44.6)$ & $11(37.9)$ & $67(57.3)$ & 0.062 \\
\hline Oral anticoagulation & $18(10.2)$ & $4(13.8)$ & $14(12.0)$ & 0.789 \\
\hline \multicolumn{5}{|l|}{ Echocardiography } \\
\hline LVOT diameter $(\mathrm{cm})$ & $2.06 \pm 0.16$ & $1.95 \pm 0.13$ & $2.09 \pm 0.15$ & $<0.001$ \\
\hline Bicuspid aortic valve & $6(4.1)$ & $1(3.4)$ & $5(4.3)$ & 0.861 \\
\hline Peak aortic jet velocity $(\mathrm{m} / \mathrm{s})$ & $3.37[0.90]$ & $3.19[0.73]$ & $3.39[0.90]$ & 0.062 \\
\hline Peak gradient $(\mathrm{mmHg})$ & $45.5[23.7]$ & $41.0[18.7]$ & $46.2[25.2]$ & 0.059 \\
\hline Mean gradient (mmHg) & 23.5 [13.9] & 21.0 [12.4] & $24.2[13.5]$ & 0.148 \\
\hline AVA $\left(\mathrm{cm}^{2}\right)$ & $1.07[0.41]$ & $1.09[0.28]$ & $1.07[0.46]$ & 0.986 \\
\hline LVEF (\%) & $59 \pm 4$ & $60 \pm 5$ & $59 \pm 4$ & 0.114 \\
\hline
\end{tabular}

Normally distributed continuous variables are presented as mean $\pm S D$, non-normal distributed continuous variables as median [IQR]. Categorical variables are presented as $n(\%)$.

Abbrevations: ARB: Angiotensin Recepter Blocker, AVA: Aortic Valve Area, BSA: Body Surface Area, LVEF: Left Ventricular Ejection Fraction, LVOT: Left Ventricular Outflow Tract

Differences in calcification activity using ${ }^{18} \mathrm{~F}$-NaF PET in patients with aortic valve stenosis Calcification activity was most frequently seen in the valvular commissures and leaflet coaptation regions. Median [IQR] TBR max $_{\text {was }} 1.93$ [0.67] and 2.29 [0.79] for females and males respectively and $T B R_{\text {mean }}$ for females and males was 1.39 [0.36] and $1.44[0.38]$. The ranges of $\mathrm{TBR}_{\max }$ and $T B R_{\text {men }}$ 


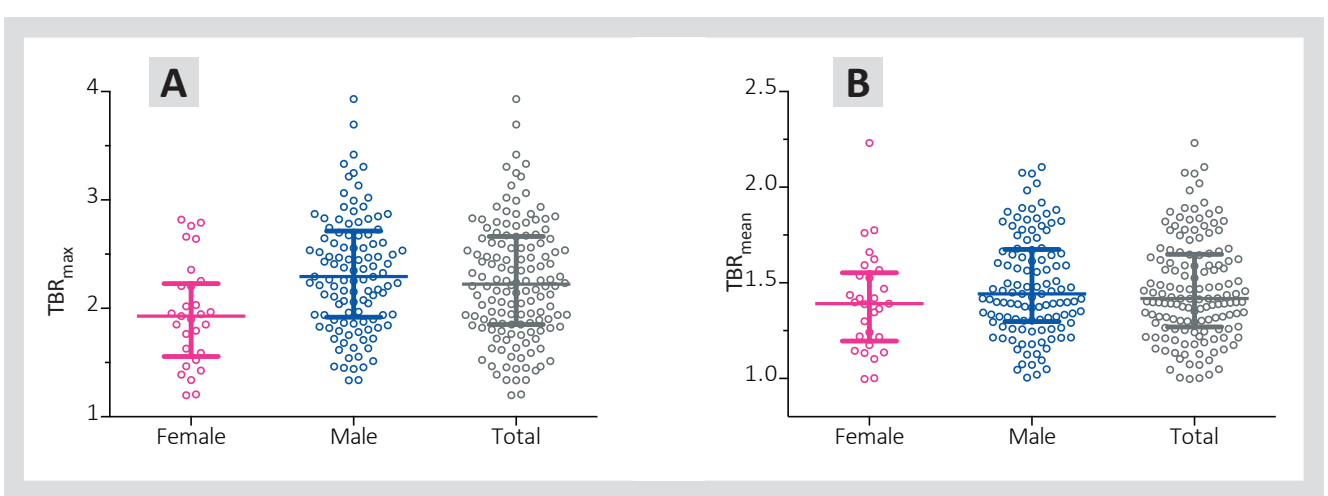

Figure 1. Calcification activity in aortic valve stenosis in males and females. A: $T B R_{\text {max }} . B: T B R_{\text {mean }}$

measurements in the total population and in the female and male subgroups are presented in Figure 1. Interobserver reproducibility was good and provided us with intraclass correlation coefficients varying from 0.828-0.991. Coefficients on variation and Bland-Altman plots are presented in Supplemental Table 1 and Supplemental Figure 1.

Univariable linear regression showed that male sex was associated with higher tracer uptake values as reflected by TBR $(\beta=0.089(0.042 ; 0.136), p<0.001)$. Further adjustment for stenosis severity and cardiovascular risk factors did not abbrogate the associations between sex and calcification activity: male sex remained associated with higher values of $\mathrm{TBR}_{\max }$ as compared with female sex $(\beta=0.068(0.013 ; 0.124), p=0.016$; Table 2$)$. Conversely, with regard to mean tracer uptake, TBR was not significantly different between males and females in all three models (Table 2).

Table 2. Associations between gender and indices of calcification activity and calcification:fibrosis ratio in patients with AS

\begin{tabular}{|c|c|c|c|c|c|c|}
\hline \multirow[t]{2}{*}{ Model } & \multicolumn{2}{|l|}{$\mathrm{TBR}_{\text {max }}$} & \multicolumn{2}{|l|}{$\mathrm{TBR}_{\text {mean }}$} & \multicolumn{2}{|c|}{ Calcification:fibrosis ratic } \\
\hline & $\beta(95 \% \mathrm{Cl})$ & P-value & $\beta(95 \% \mathrm{Cl})$ & P-value & $\beta(95 \% \mathrm{Cl})$ & P-value \\
\hline 1 & $\begin{array}{l}0.089 \\
(0.042 ; 0.136)\end{array}$ & $<0.001$ & $\begin{array}{l}0.024 \\
(-0.010 ; 0.057)\end{array}$ & 0.161 & $\begin{array}{l}0.516 \\
(0.292 ; 0.741)\end{array}$ & $<0.001$ \\
\hline 2 & $\begin{array}{l}0.067 \\
(0.017 ; 0.118)\end{array}$ & 0.009 & $\begin{array}{l}0.014 \\
(-0.023 ; 0.051)\end{array}$ & 0.458 & $\begin{array}{l}0.407 \\
(0.164 ; 0.649)\end{array}$ & 0.001 \\
\hline 3 & $\begin{array}{l}0.068 \\
(0.013 ; 0.124)\end{array}$ & 0.016 & $\begin{array}{l}0.021 \\
(-0.020 ; 0.061)\end{array}$ & 0.319 & $\begin{array}{l}0.261 \\
(0.006 ; 0.516)\end{array}$ & 0.045 \\
\hline
\end{tabular}

Values are regression coefficients (and their 95\% confidence intervals). Outcome variables are log-transformed. Model 1: univariable model including sex. Model 2: model 1 plus adjustment for maximum velocity aortic valve and LVOT diameter. Model 3: model 2 plus age, BSA, presence of coronary artery disease, diabetes mellitus, hypertension, hyperlipidaemia and smoking
Calcification:fibrosis ratio and calcification activity in females and males with aortic

valve stenosis

To explore the predominant process in the valve, both calcification and fibrosis volume parameters measured on CT were integrated in a calcification:fibrosis ratio. Overall, the ratio (median [IQR]) was 1.048 [1.5130] in males, and 0.6726 [1.0548] in females. A ratio $>1$ indicated a trend towards calcification as the predominant phenotype, and a ratio $<1$ represented a trend towards greater fibrosis. Unadjusted linear regression revealed that male sex was associated with a higher calcification:fibrosis ratio (Table 2). Moreover, upon dichotomizing the calcification:fibrosis ratio, we found a significant difference between males and females with a ratio $>1$ (53\% for males and $27 \%$ for females respectively, $p=0.018$ ), indicating a predominance of a fibrotic phenotype in the valves of females (Figure 2).

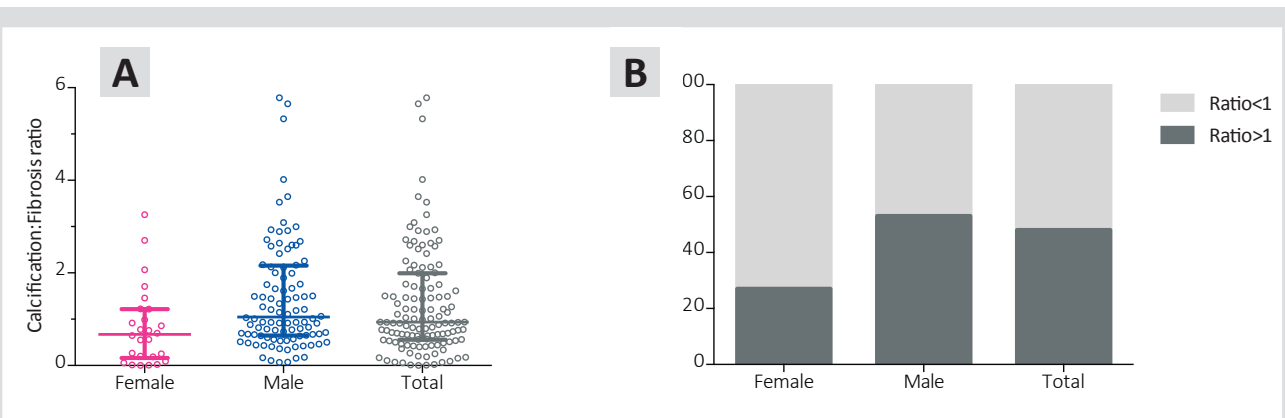

Figure 2. Calcification:fibrosis ratio in aortic valve stenosis in females (pink), males (blue) and the total population (grey). A: Calcification:fibrosis ratio, B: presence (\%) of ratio $<1$ (light grey) and ratio $>1$ (dark grey)

Given the difference in predominant processes of fibrosis and calcification in females and males, we expected a ratio $>1$ to correspond with higher TBR values. Therefore, we explored whether values of TBR and TBR corresponded with higher calcification:fibrosis ratios and found that both $\mathrm{TBR}_{\max }$ and $\mathrm{TBR}_{\text {man }}$ were significantly higher in patients with a ratio $>1$ (both p<0.001), and thus with a calcific phenotype. Figure 3 illustrates representative images of calcification activity and different valve phenotypes.

\section{DISCUSSION}

Using a combined ${ }^{18} \mathrm{~F}$-sodiumfluoride PET/CT approach, we investigated calcification activity and the calcification:fibrosis ratio and found that females tend to show a fibrotic phenotype, whilst the calcification phenotype is predominantly present in males with AS. Calcification activity seemed higher in males when considering $\mathrm{TBR}_{\text {max }}$, but we could not find a significant difference when considered $\mathrm{TBR}_{\mathrm{me}}$

\section{Sex-specific differences in calcification activity: present and relevant?}

In accordance with previous studies, males presented a higher calcification burden than females in our study. However, whether these sex differences result in a discrepancy in calcification activity, as measured by ${ }^{18} \mathrm{~F}-\mathrm{NaF}$ uptake, has not been addressed in curren iterature. ${ }^{18} \mathrm{~F}$-sodiumfluoride is a marker of calcification activity, and is incorporated on the 


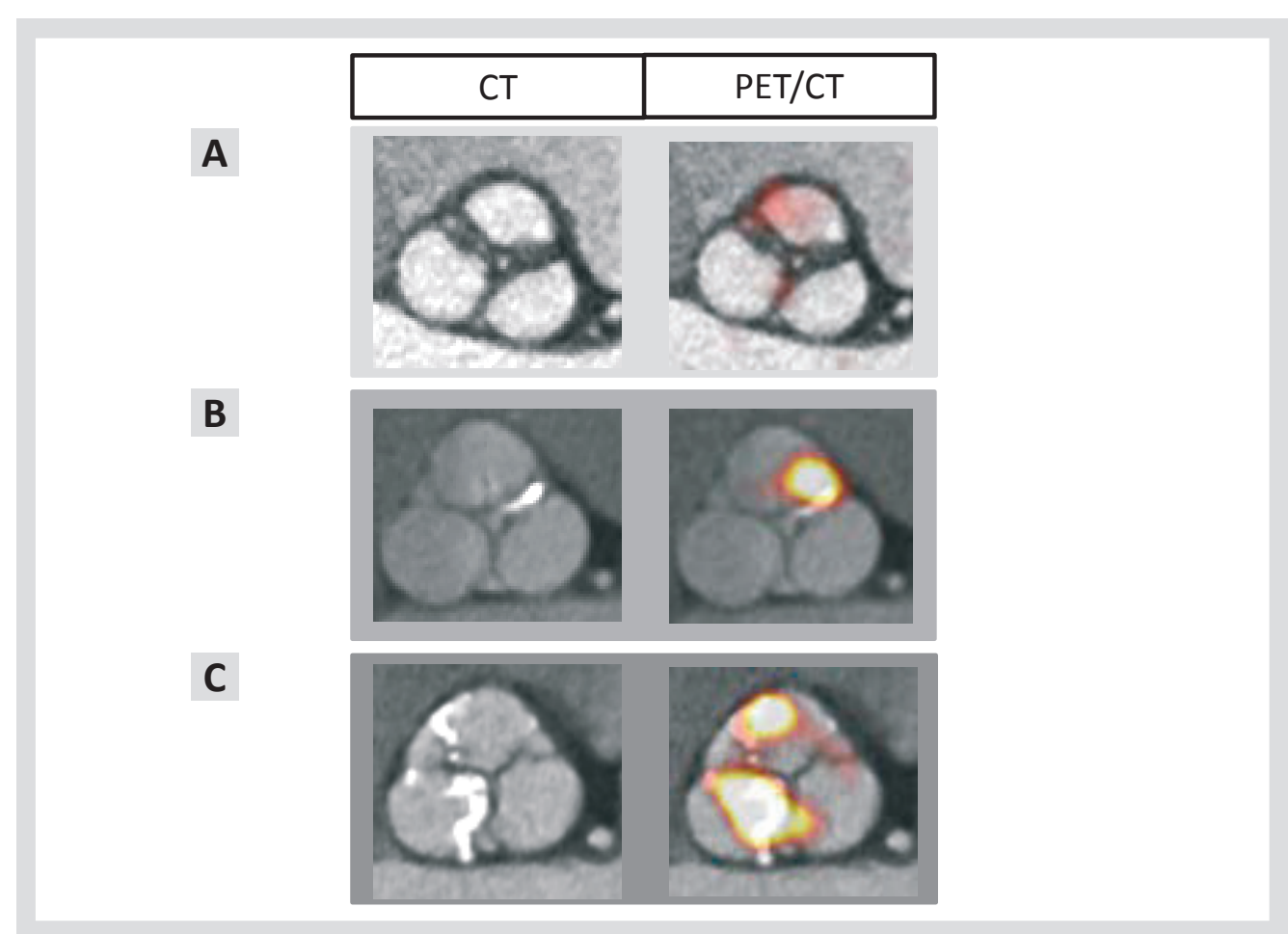

Figure 3. Contrast-enhanced CT and PET images of aortic valves with A: predominant fibrosis (ratio<1), B: balanced calcification:fibrosis ratio (ratio 1), C: predominant calcification (ratio $>1$ )

surface of key components in vascular calcification: hydroxyapatite crystals. It is supposed to be able to differentiate between active and quiescent calcification, related to the available surface area of hydroxyapatite to which the ${ }^{18} \mathrm{~F}-\mathrm{NaF}$ ions can adsorb. ${ }^{3,12,25} \mathrm{Thus},{ }^{18} \mathrm{~F}-\mathrm{NaF}$ uptake is greater in areas of microcalcification (developing calcification) when compared to areas with established calcification when the hydroxyapatite core is internalized and therefore unavailable for binding. With that, it provides additional information to the calcium score, which quantifies regions of established calcification rather than developing calcification. $12,13,26$ In our study, we found that male sex was associated with higher calcification activity as revealed by $T B R_{\text {max }}$. However, this association diminished when TBR man $_{\text {was }}$ considered. Several explanations can be proposed for this discrepancy, starting with an insufficient power to distinguish true differences. Second, calcification activity is not homogenously present in the valve area. Rather, it is distributed throughout the valve, with gross areas of calcification and areas with smaller spots of calcification. ${ }^{18} \mathrm{~F}-\mathrm{NaF}$ uptake is highest in these areas of developing calcification. $\mathrm{TBR}_{\text {mean }}$ generalizes uptake in the valve, whereas $\mathrm{TBR}_{\max }$ reflects the area with with the highest activity in the valve ${ }^{15}$, and might therefore more sensitive to detect differences and change in activity as compared to $\mathrm{TBR}_{\text {mean }}$. However, there is preliminary evidence that calcification activity, as reflected by $\mathrm{TBR}_{\text {mean }}$ has potential to predict AS progression. ${ }^{12}$ Males show a higher calcification burden and progression of calcification, and therefore, their maximum activity in regions with developing calcification as reflected by TBR migxt $_{\text {migh }}$ higher than values seen in females. Data on the value of $\mathrm{TBR}_{\text {max }}$ the established method of measurement in oncology, in prediction of AS progression is unknown at present. These findings merit further investigation in longitudinal studies investigating progression of AS and the clinical relevance of calcification activity differences.

\section{Calcification:fibrosis ratio in non-invasive imaging: a fibrotic phenotype is predominant}

in females

Aortic valve calcification (AVC) is a key component in development and progression of AS. Yet, the disproportion of AVC and AS hemodynamic severity in females has been a critical issue in clinical practice. Also, differences in calcium burden persist despite correction for patient size or aortic valve area. Sex-specific thresholds for AVC burden are incorporated in the most recent guidelines to identify severe AS in females and males. ${ }^{16}$ Moreover, sex-specific thresholds for AVC provide powerful prognostic information. ${ }^{17}$ Despite growing knowledge of AS pathophysiology, serious gaps in knowledge persisted for a long time, especially regarding the sex specific pathophysiology. Recently, Simard et al presented results suggesting that a predominant role of valvular fibrosis in women might provide explanation for differences in AVC thresholds. They investigated calcification:fibrosis ratio in patients with severe AS, quantifying calcification and fibrosis using non-contrast enhanced $\mathrm{CT}$ and histology of valvular tissue. ${ }^{11}$ Our results are in accordance with those results: we found calcification:fibrosis ratio $>1$ in $53 \%$ of males, while a ratio $<1$ was found in $73 \%$ of females, suggesting a dominant contribution of non-calcified tissue to AS severity in females and thus towards a fibrotic phenotype in females as opposed to a calcific phenotype in males. Our study adds information by including patients with varying degrees of AS and by using a combined non-invasive clinical imaging method.

The question why females and males show differences in pathophysiology of AS remains largely hypothetical. The role of both estrogen and androgen pathways are controversial. On the one hand, male sex has been shown to be a risk factor for $\mathrm{AS}, 18,19$ but information regarding the involvement of the androgen signaling pathway in vascular calcification is contradictory. It was shown to induce cellular calcification of vascular smooth muscle cells (VSMC) in mice models, whilst another study showed an inhibitory effect in human VSMC calcification. ${ }^{20,21}$ The effect of androgen pathways in AS has not been studied. On the other hand, an in vitro study presented estrogen as a driving factor for calcification by driving VSMC differentiation to osteoblast-like cells, whilst in human VSMCs, estrogen was found to have an inhibitory effect on calcification. ${ }^{22,23}$ Moreover, it is suggested that the loss of estogen during menopause may contribute to the trend of females developing heart disease later in life. ${ }^{19,24}$ The explanation of the divergent patterns of AS development and progression presumably is more complex though and will need further investigation.

\section{Limitations}

Several limitations of our study merit attention. First, the cross-sectional character of this study makes it impossible to provide data on progression of AS. This information would be highly valuable to determine whether imaging women and men demonstrate differences in rate of progression in valve calcium or stenosis. ${ }^{12}$

Second, the size of the female group is limited, which might have resulted in an insufficient power to detect true changes in TBR between males and females. It remains questionable whether large numbers would lead to a clinically meaningful difference, but it would provide us with the opportunity to perform stratified analyses in subgroups of AS severity. Third, we were the first to determine valvular fibrosis volume using contrast-enhanced CT. It would be valuable to confirm these results in histology, which is rather impossible in patients with mild or moderate AS without a surgical indication. Our results regarding the calcification:fibrosis ratio are in accordance with Simard et al though. ${ }^{11}$ 
Clinical implications

We present the first study to using PET/CT imaging to describe potential gender differences in the pathophysiology of aortic valve stenosis, focusing in particular on calcification activity and fibrosis. The findings of the present study confirm sex-specific predominant processes in aortic valve stenosis, ${ }_{11}^{11}$ suggesting that females with AS develop more fibrosis relative to AVC and thus a fibrotic phenotype, whilst the opposite phenomenon (calcification over fibrosis) is present in males (calcific phenotype). It has important clinical implications, given the discordance of As hemodynamic severity and AVC seen in daily practice, when fibrosis is not incorporated in clinical evaluation. Furthermore, we identified regions of developing (micro)calcification by using non-invasive, combined approach of PET and contrast-enhanced CT. Although further confirmation of our techniques to quantify both calcification activity and fibrosis is needed in longitudinal studies, these findings suggest that using ${ }^{18} \mathrm{~F}-\mathrm{NaF}$ PET/CT might be a suitable way to investigate this issue. Moreover, using ${ }^{18} \mathrm{~F}-\mathrm{NaF} \mathrm{PET} / \mathrm{CT}$ enables us to investigate the processes in more detal in early phases of AS, whereas histologic assessment is hampered by the need for valve tissue, mostly derived from surgery in severe AS

Ultimately, these findings hold potential to develop pharmacological treatments with sex-specific predominant processes. Nonetheless, further research to understand the complex molecular pathways driving development and progression of AS is crucial.

\section{CONCLUSION}

Males and females with aortic valve stenosis show a difference in dominant processes underlying the disease. Female gender is associated with a fibrotic phenotype in AS, whereas male gender is associated with a calcific phenotype. Whether sex-differences in calcification activity exist is questionable though. These findings merit further investigation in longitudinal studies investigating progression of aortic valve stenosis and in the development of effective pharmacological interventions reducing disease progression.
REFERENCES

nosis incidence and $P$. Population-wide trends in aortic steRoberts WC, Ko JM. Frequency by decades of unicuspid, bicuspid, and tricuspid aortic valves in adults having isolated aortic valve replacement for aortic stenosis, with or 2 without associated aortic regurgitation. Circulation Som, 111.9205.

awade TA, Newby DE, Dweck MR. Calcification in Aortic Peeters F, Meex SIR, Dweck MR et al Calcific aortic valve stenosis: hard disease in the heart: A biomolecular approach towards diagnosis and treatment. Eur HeartJ 2017 Carabello BA, Paulus WJ. Aortic stenosis. Lancet 2009;373:956-66

Otto CM, Kuusisto J, Reichenbach DD, Gown AM, O'Brien D. Characterization of the early lesion of 'degenerative' chemical studies. Circulation 1994;90:844-53.

Hervault M, Clavel M-A. Sex-related differences in calcific aortic valve stenosis: pathophysiology, epidemiology, etioogy, diagnosis,

essika-Zeitoun D et al. Sex differences in aortic valve calcification measured by multidetector computed tomography in aortic stenosis. Circ Cardiovasc Imaging 2013;6:40-7.

Clavel MA, Messika-Zeitoun D, Pibarot P et al. The complex rading. new insights from combined $D$ oppler diol 2013;62:2329-38.

10. Clavel MA, Pibarot P, Messika-Zeitoun D et al. Impact of aortic valve calcification, as measured by MDCT, on surviva in patients with aortic stenosis: results of an internation

11. Simard L, Cote N, Dagenais $F$ et al. Sex-Related Discordance Severity of Aortic Stenosis: Is Valvular Fibrosis the Explanation? Circ Res 2017;120:681-691.

2. DweckMR, Jenkins WS, Vesey AT et al. 18F-sodium fluoride uptake is a marker of active calcification and disease promaging 2014:7:371-8.

13. Irkle A, Vesey AT, Lewis Dy et al. Identifying active vascular microcalcification by (18)F-sodium fluoride positron emission tomography. Nat Commun 2015;6:7495,

14. Baumgartner $\mathrm{H}$, Hung J, Bermejo $\mathrm{J}$ et al. Recommendations on the Echocardiographic Assessment of Aortic Valve Ste Cardiovascular Imaging and the American Society of Echocardiography. J Am Soc Echocardiogr 2017:30:372-392.

15. Pawade TA, Cartlidge TR, Jenkins WS et al. Optimization and Reproducibility of Aortic Valve 18F-Fluoride Positron Emission Tomography in Pate Viv With Aortic Stenosis. Cir

16. Baumgartner H, FalkV, Bax JJ et al. 2017 ESC/EACTS Guidelines for the management of

17. Pawade T, Clavel MA, Tribouilloy C et al. Computed Tomogaphy Aortic Valve Calcium Scoring in Patients With Aortic Stenosis. Circ Cardiovasc Imaging 2018;11:e007146.
Stewart BF, Siscovick D, Lind BK et al Clinical factors

8. Stewar $B$, Siscovick D, Lind Bk et al. Cinical factors associHealth Study. Am Coll Cardiol 1997:29:630-4.
Sritharen Y Enriquez-Sarano M, Schaff HV, Casaclang-Vercosa G. Miller JD. Pathophysiology of Aortic Valve Stenosis: (s It Both Fibrocalcific and

Son BK, Akishita M, limina $\mathrm{K}$ et al. Androgen receptordependent transactivation of growth arrest-specific gene 6 cification J Biot Chen 2010;2857537-44.

Zhu D, Hadoke PW, Wu J et al. Ablation of the androge receptor from vascular smooth muscle cells demonstrates 2016;6:24807.

Mcrobb LS, McGrath KCY, Tsatralis Tet al. Estrogen Rece tor Control of Atherosclerotic Calcification and Smoot Thromb Vasc Biol 2017;37:1127-1137.

Nanao-Hamai M, Son BK, Hashizume I, Ogawa S, Akishita M. Protective effects of estrogen against vascular calcification via estrogen receptor alpha-dependent growth arresspecific gene 6 transactivation.

N WISE) Study: Part l: gender differences in traditional and novel risk factors, symptom evaluation, and gender-optimized diagnostic strategies. JAm Coll Cardiol 2006;47:54-S2O Derin T, Richter U, Bannas $P$ et al. Feasibility of 18F-sodium Nucl Med 2010;51:862-5.

, Shah AS et al. Valvular (18)F-Fluoride and (18)F-Fluorodeoxyglucose Uptake Predict Disease Progression and Clinical Outcome in Patients With Aortic Stenosis. J Am Coll Cardiol 2015;66:1200-1. 
Supplemental Table 1. Interobserver variation

\begin{tabular}{|c|c|c|c|c|}
\hline & Mean difference & Lower limit & Upper limit & ICC \\
\hline Blood pool SUV mean & -0.0140 & -0.2476 & 0.2196 & 0.970 \\
\hline$A V S U V_{\text {mean }}$ & -0.2019 & -0.7310 & 0.3272 & 0.929 \\
\hline$A V S U V_{\text {max }}$ & -0.0531 & -0.4933 & 0.3870 & 0.991 \\
\hline$A \vee T B R_{\text {mean }}$ & -0.1446 & -0.4793 & 0.1902 & 0.828 \\
\hline$A V T B R_{\max }$ & -0.0480 & -0.5589 & 0.4629 & 0.972 \\
\hline
\end{tabular}

Abbreviations: AV: aortic valve, ICC: intraclass correlation coefficient, SUV: standard uptake value, TBR: target to background ratio

\section{SUPPLEMENTAL MATERIAL}




\section{Biomarkers associated with early aortic valve calcification: should we focus on sex specific processes?}

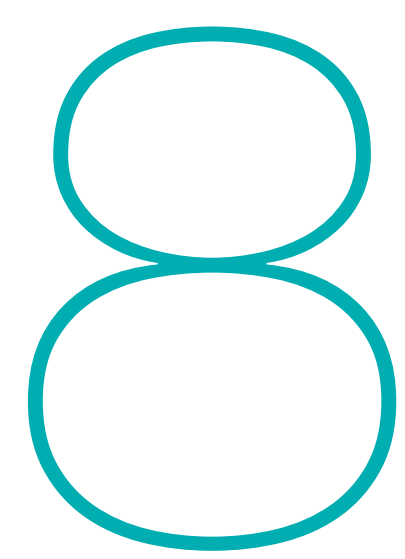

Frederique E.C.M. Peeters ${ }^{1}$, Elton A.M.P. Dudink ${ }^{1}$, Bob Weijs ${ }^{1}$, Larissa Fabritz ${ }^{2}$, Winnie Chua', Bas L.J.H. Kietselaer ${ }^{1,3}$, Joachim E. Wildberger ${ }^{4}$, Steven J.R. Meex ${ }^{5}$, Paulus Kirchhof ${ }^{2}$, Harry J.G.M. Crijns ${ }^{1}$, Leon J. Schurgers ${ }^{6}$

${ }^{1}$ Maastricht University Medical Center+ and CARIM, department of Cardiology, Maastricht, the Netherlands,

${ }^{2}$ University of Birmingham, Institute of Cardiovascular Sciences, Birmingham, United Kingdom,

${ }^{3}$ Current affiliation: Zuyderland Medical Center Heerlen/Sittard, the Netherlands,

${ }^{4}$ Maastricht University Medical Center+ and CARIM, department of Radiology and Nuclear Medicine, Maastricht, the Netherlands,

${ }_{5}^{5}$ Maastricht University Medical Center+, department of Clinical Chemistry, Maastricht, the Netherlands,

${ }^{6}$ Maastricht University and CARIM, department of Biochemistry, Maastricht, the Netherlands 


\section{ABSTRACT}

Background: Circulating biomarkers are useful in detection and monitoring of cardiovascular diseases. However, their role in aortic valve disease is unclear. Mechanisms are rapidly elucidated and sex differences are suggested to be involved. Therefore, we sought to identify biomarkers involved in aortic valve calcification (AVC) stratified by sex.

Methods: Blood samples of 34 patients with AVC (without further overt cardiovascular disease including absence of hemodynamic consequences of valvular calcification) were compared with 136 matched controls. AVC was determined using computed tomography calcium scoring. Circulating biomarkers were quantified using a novel antibody-based method (Olink Proseek Multiplex Cardiovascular Panel I) and 92 biomarkers were compared between patients with and without AVC.

Results: In the overall population, Interleukin-1 Receptor Antagonist and pappalysin-1 were associated with increased and decreased odds of having AVC. These differences were driven by the male population (IL1RA: OR 2.79(1.16-6.70), p=0.022; PAPPA: OR 0.30(0.11-0.84), p=0.021) Furthermore, TNF-related activation-induced cytokine and fibroblast growth factor-23 were associated with decreased odds of having AVC, and monocyte chemotactic protein-1 was associated with increased odds of having AVC (TRANCE: OR 0.32(0.12-0.80), $\mathrm{p}=0.015$; FGF23: OR $0.41(0.170-0.991), p=0.048 ; M C P 1:$ OR 2.64(1.02-6.81), $p=0.045)$. In contrast, galanin peptides and ST2 were associated with increased odds of having AVC in females (GAL: OR 12.38(1.31116.7), $\mathrm{p}=0.028$; ST2: OR 13.64(1.21-153.33), $\mathrm{p}=0.034)$.

Conclusions: In this exploratory study, we identified biomarkers involved in inflammation, fibrosis and calcification which were associated with having AVC. Furthermore, we found sex-related differences in these associations. Biomarkers involved in fibrosis showed higher expression in females, whilst biomarkers involved in inflammation and calcification associated with AVC in males.

Key words: aortic valve calcification; biomarkers; sex-specific; fibrosis; inflammation

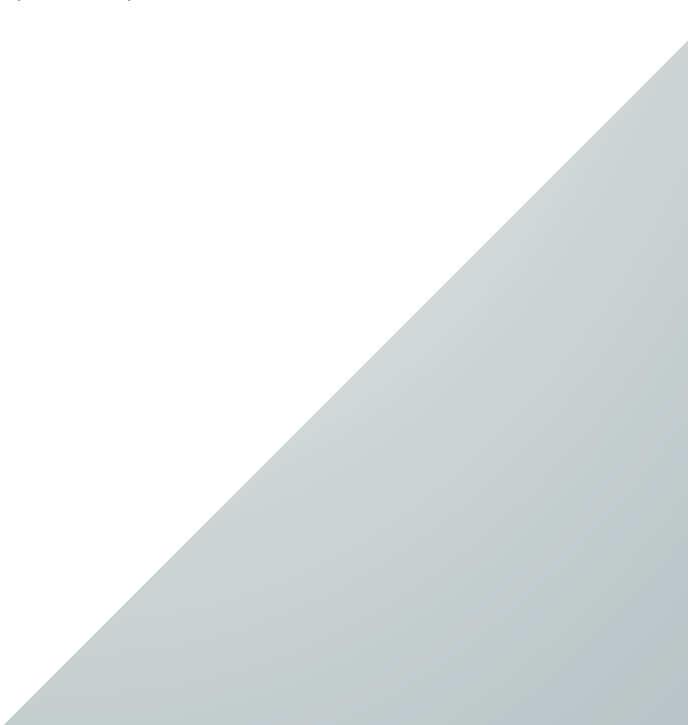

INTRODUCTION

Aortic valve calcification (AVC) is a major determinant in leaflet stiffening and progression of aortic valve disease. Whereas aortic valve disease used to be considered a passive and degenerative process, it is now appreciated to be an active process with involvement of multiple cellular and molecular pathways in inflammation, fibrosis and calcification. Pathophysiological mechanisms involved in the initiation and progression of aortic valve disease are rapidly elucidated, but their exact contribution and extent of their involvement remain to be investigated. ${ }^{1,2}$ Moreover, sex specificity of processes involved are suggested to be present in aortic valve disease, yet are largely unresolved. ${ }^{3}$ Insight into molecular calcification processes may help to define appropriate interventions to halt or reduce progression.

Once present, AVC progresses and development of hemodynamically evident aortic valve disease is a common feature, requiring regular monitoring using echocardiography (and computed tomography). Addition of biomarkers to optimize risk assessment of progressive diseases would be useful from the initial phase onwards. The ESC guidelines only integrate a possible role for NT-proBNP in timing of aortic valve replacement though. ${ }^{4}$ This might be due to the fact that most studies focus on the identification of biomarkers in patients with advanced aortic valve disease.

Therefore, we aimed to identify circulating biomarkers holding potential for further investigation in the early phase of aortic valve calcification in a low risk population.

\section{METHODS}

\section{Study population}

In this cross-sectional observational study, 390 patients without clinically overt vascular disease (other than lone atrial fibrillation (AF)) who underwent cardiac Computed Tomography (CT) (January 2008-March 2011) in the work-up for pulmonary vein isolation ( $n=115)$ or general screening $(n=275)$ were selected. These patients were age, sex and PROCAM-matched as described previously. ${ }^{5}$ Within this group of patients, EDTA-plasma was available in 180 patients. Ten patients were not included in further analyses based on a large number of values below the limit of detection (valid measurement for $<85 \%$ of the proteins), and thus 170 ( $n=48$ AF, $n=122$ sinus rhythm) constituted the final population for the current study. This study was approved by the Institutional Review Board.

\section{Computed Tomography}

All patients underwent a non-contrast enhanced coronary calcium scan as described previously, performed on a Philips Brilliance 64-slice MSCT scanner (Brilliance 64; Philips Healthcare, Best, the Netherlands) or a $2^{\text {nd }}$ generation Dual source CT scanner (Siemens Somatom Definition Flash 2*128-slice, Siemens Healthineers, Forchheim, Germany). ${ }^{5}$ Quantitative assessment (expressed as Agatston scores) of aortic valve calcification (AVC) was performed by 2 independent observers Presence of AVC was defined as Agatston score $>0$.

\section{Biomarkers}

Proteins were quantified by real-time PCR in all EDTA-plasma samples using the Olink Proseek Multiplex Cardiovascular I kit (Olink Proteomics, Uppsala, Sweden), as described previously. Interleukin 4 (IL4), Natriuretic Peptides B (BNP) and Melusin (ITGB1BP2) were excluded from further analyses due to low call rates (valid measurement in $<85 \%$ of samples). Values below the Limit of Detection (LOD) were replaced by the LOD value (http://www.olink.com/data-you-can-trust/validation/). 
Data from the panels were normalised to the median of 0 for each protein, enabling comparisons between measurements from different panels. The panel provides NPX-values which allow for relative quantification comparisons for the same protein across samples.

\section{Statistical analyses}

Statistical analyses were performed using SPSS version 22 (IBM Corp, Armonk, NY). Normally distributed continuous variables are expressed as mean \pm standard deviation (SD) and compared using the independent samples t-test, non-normally distributed continuous variables as median interquartile range; IQR] and compared using the Mann-Whitney U test. Categorical variables are expressed as absolute numbers and percentages and tested using the Fishers exact test.

Logistic regression adjusted for age, presence of AF (and sex when appropriate) was used to determine the association between biomarkers and calcification with AVC or no AVC as the outcome. Odds ratios and $95 \%$ confidence intervals (CI) were calculated and $p<0.05$ was considered significant.

\section{RESULTS}

\section{Aortic valve calcification on $\mathrm{CT}$}

AVC was present in 34 patients: 11 females, 23 males (median [IQR] Agatston scores of the total, female and male populations were 11.3 [47.6], 15.8 [69.2] and 11.2 [40.8] respectively). In general, patients with AVC were older than patients without AVC (mean age $59 \pm 6$ vs $53 \pm 10$ years in patients with versus without $A V C, p<0.001)$. Other baseline characteristics were not significantly different (Supplemental Table 1).

\section{Biomarkers and valvular calcification}

Supplemental table 1 shows the age, sex and AF adjusted OR (and $95 \% \mathrm{Cl}$ ) of all biomarkers. In the total population, Interleukin 1 receptor antagonist protein (IL1RA) was associated with increased odds of having AVC (OR 2.29 (1.13-4.65), $p=0.022)$. Furthermore, pappalysin-1 (PAPPA) was associated with decreased odds of having AVC (OR 0.37 (0.16-0.87), p=0.023) (Figure 1A)

The abovementioned differences of ILIRA and PAPPA were driven by the male population (IL1RA: OR 2.79 (1.16-6.70), $p=0.022$ and PAPPA: OR 0.30 (0.11-0.84), $p=0.021$ respectively). Furthermore, TNF-related activation-induced cytokine (TRANCE) and fibroblast growth factor 23 (FGF23) were lower and monocyte chemotactic protein 1 (MCP1) was higher in males with AVC than without AVC (TRANCE: OR 0.32 (0.12-0.80), $p=0.015$; FGF23: OR 0.41 (0.45-2.29), $p=0.048$ and MCP1: OR 2.64 (1.02-6.81), $p=0.045$ ) (figure 1).

In the female population, galanin peptides (GAL) and ST2 protein (ST2) odds ratios were higher in females with AVC than in females without AVC (GAL: OR 12.38 (1.31-116.69), $p=0.028$; ST2: OR 13.64 (1.21-153.33), $p=0.034$ ) (Figure 1A).

Distributions of biomarkers significantly associated with AVC are shown in Figure $1 \mathrm{~B}$.

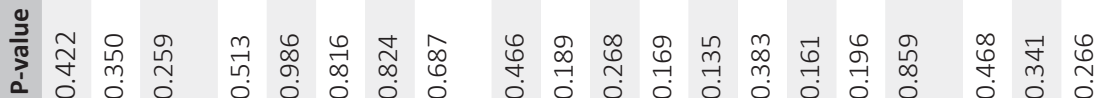

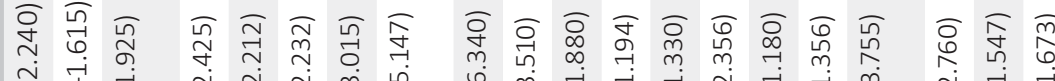

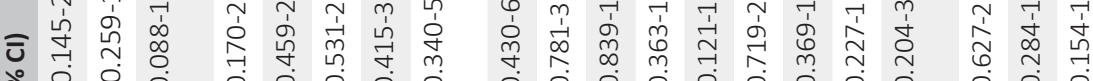
芦送

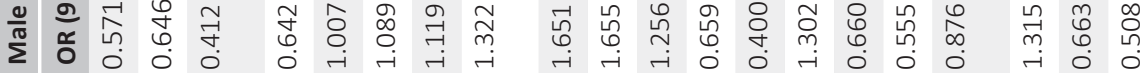

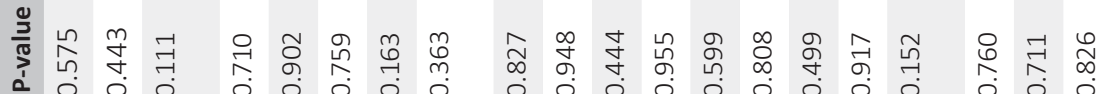

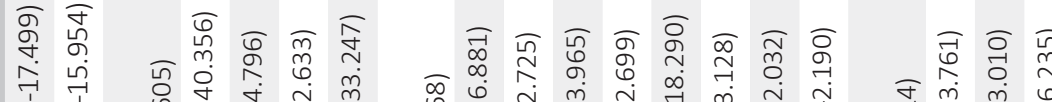

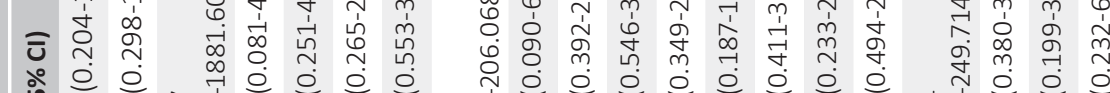

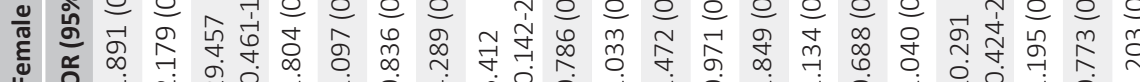

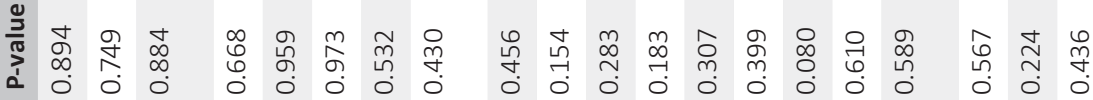

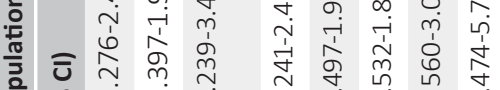

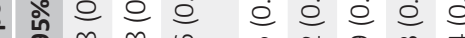

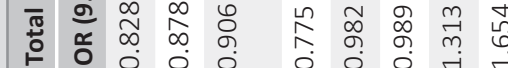

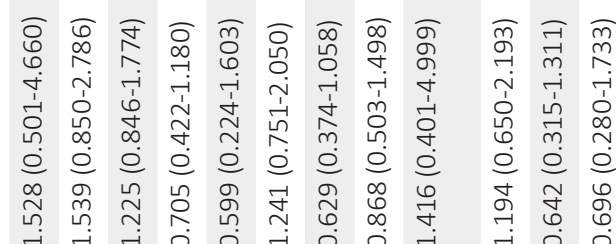




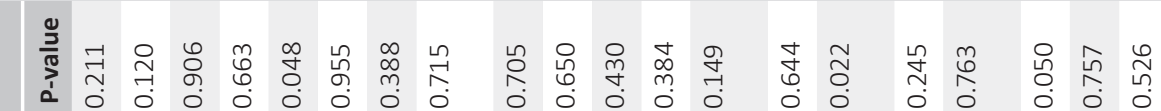

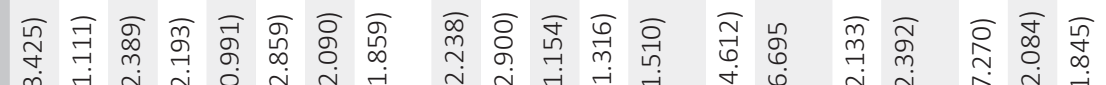

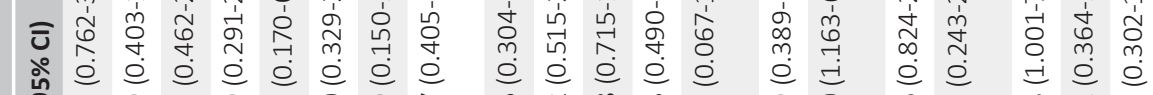

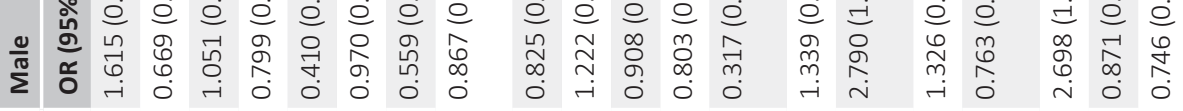

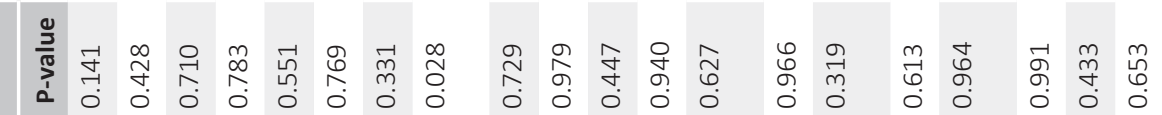

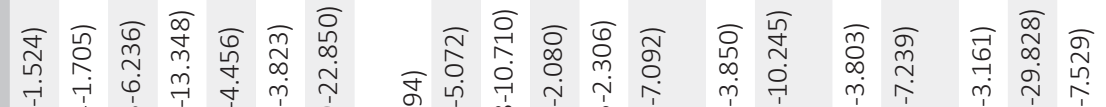

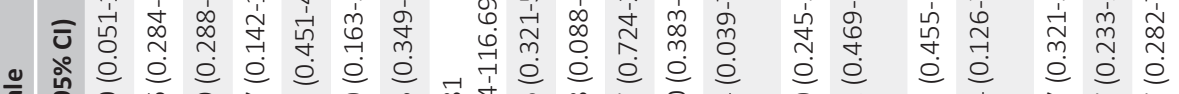

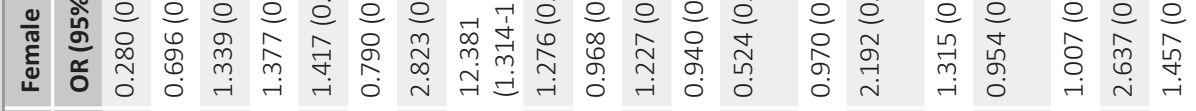

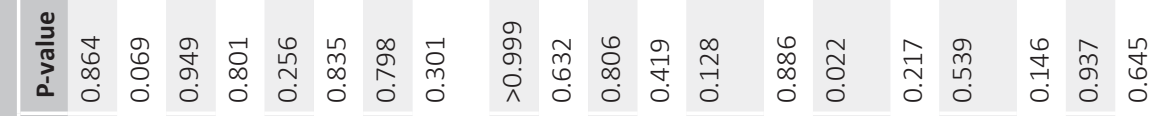

Б

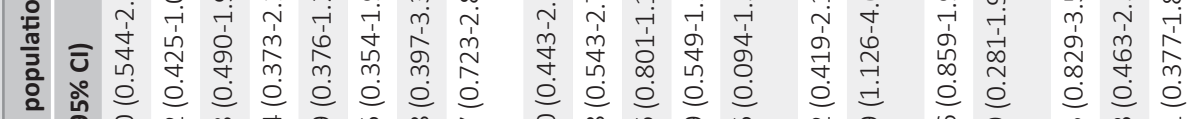

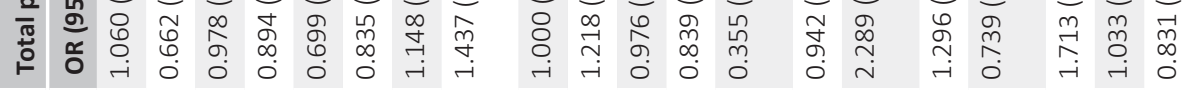

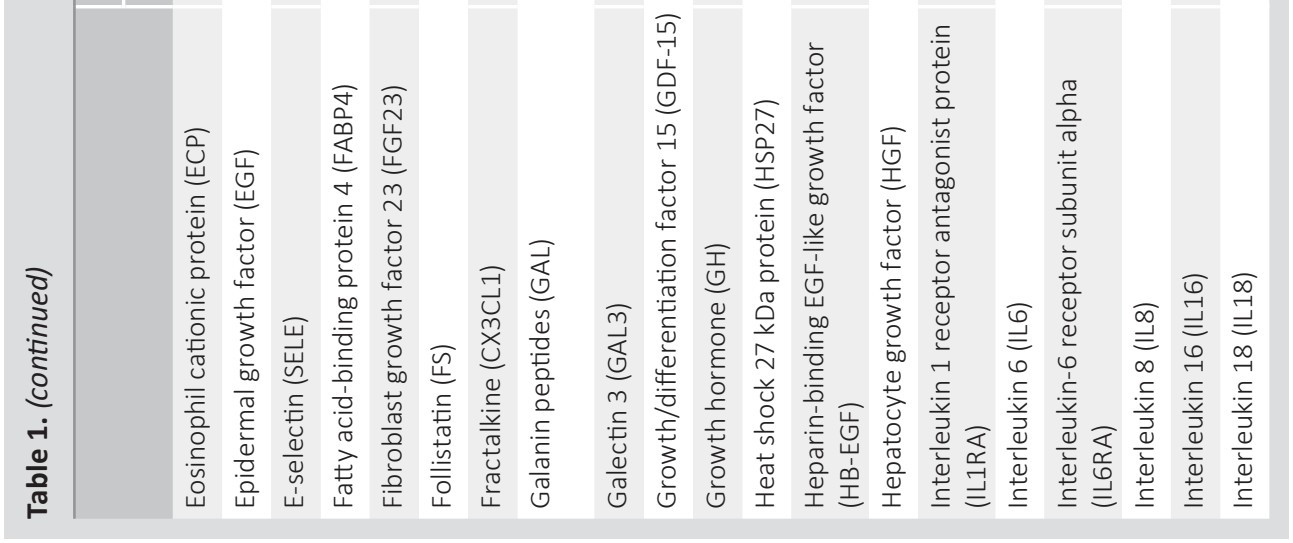

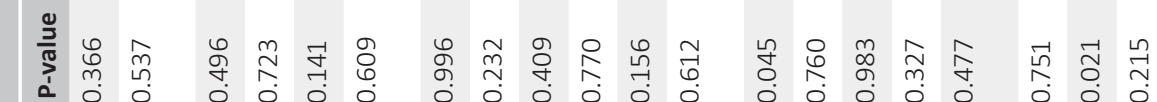

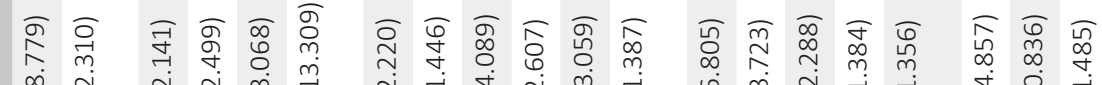

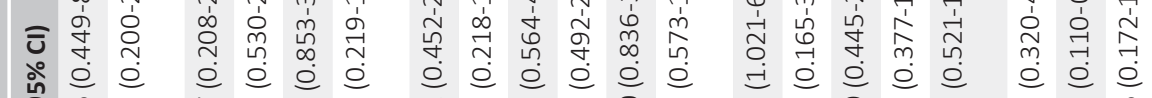

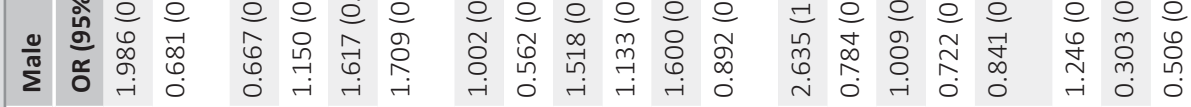

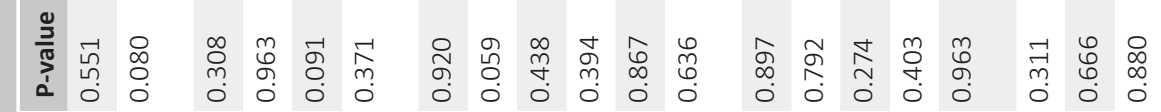

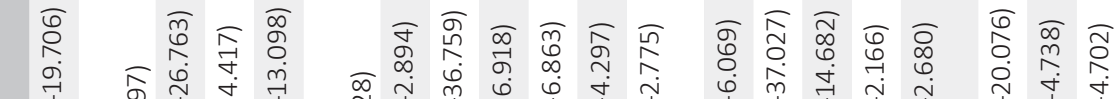

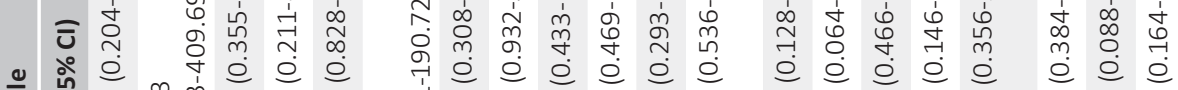

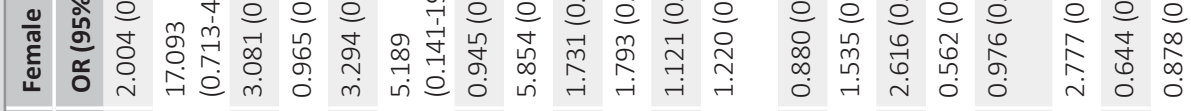

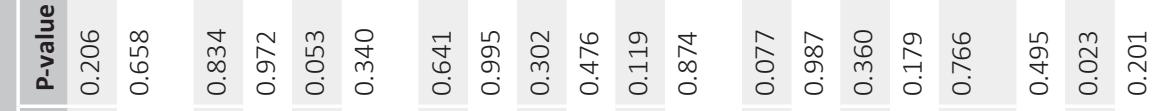

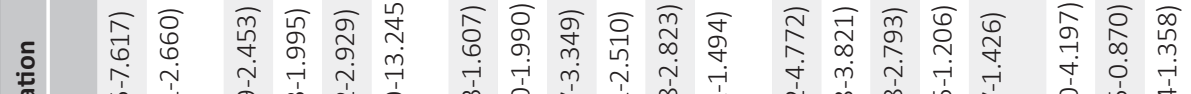

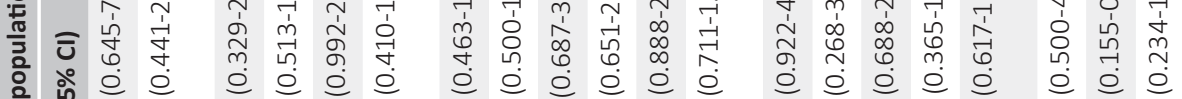

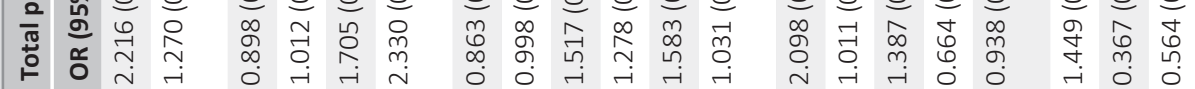

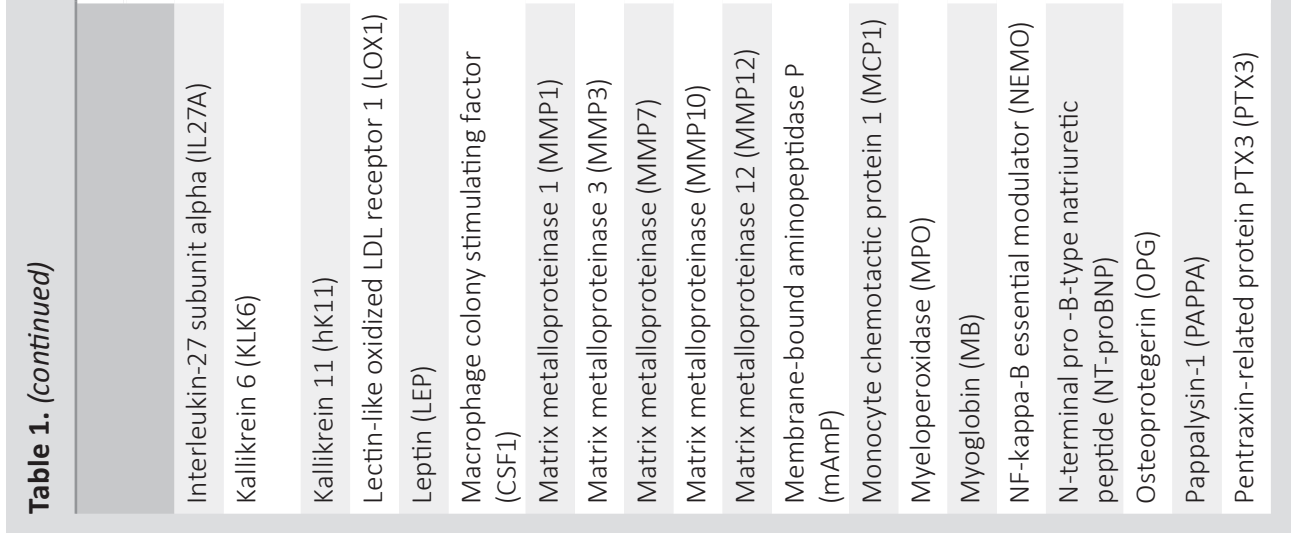




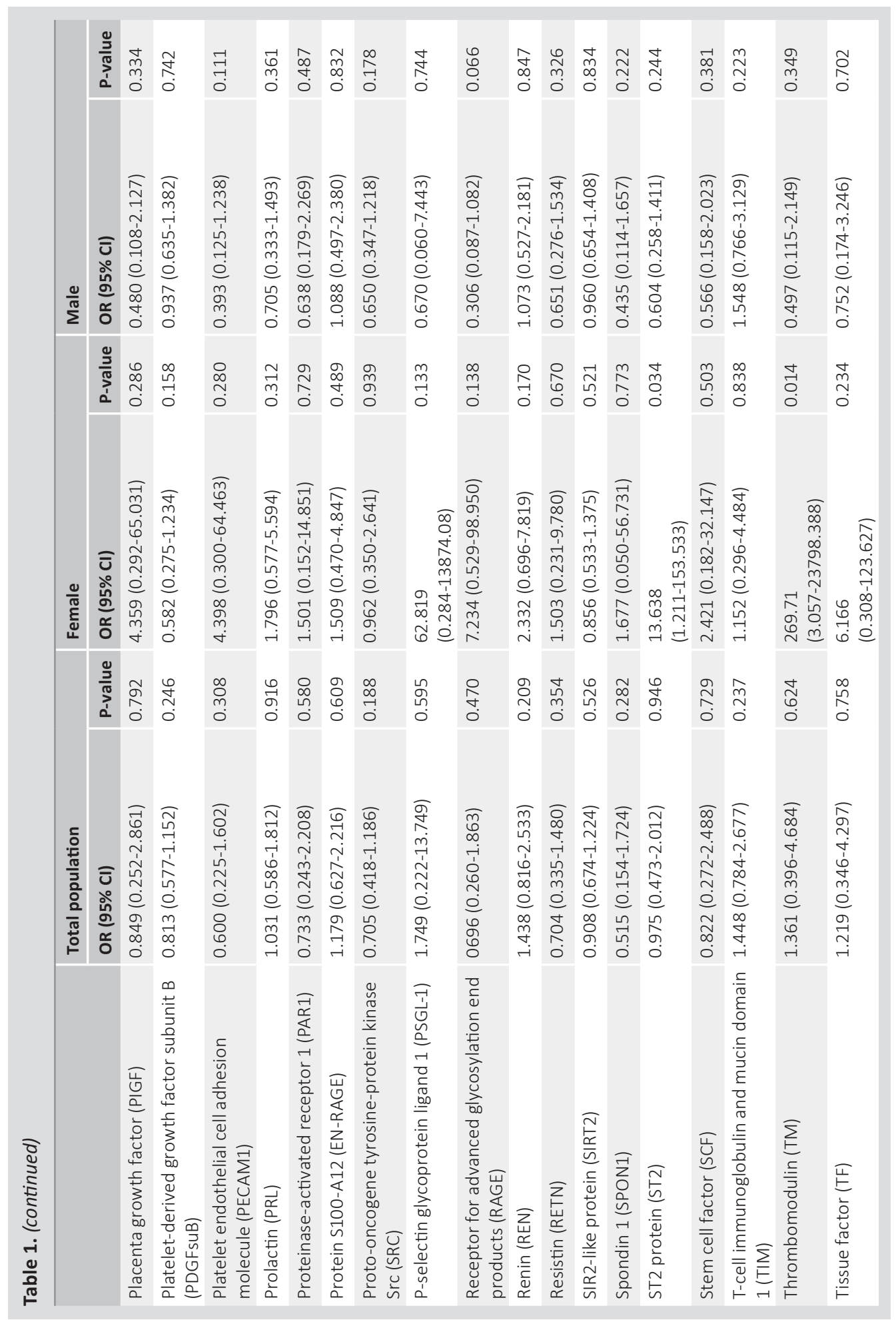

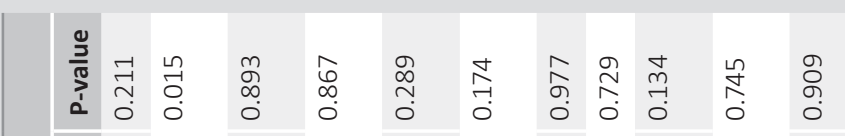

岛高

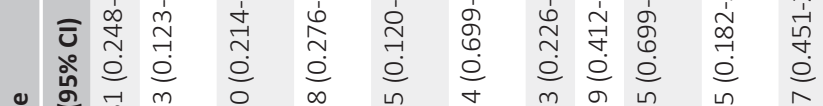

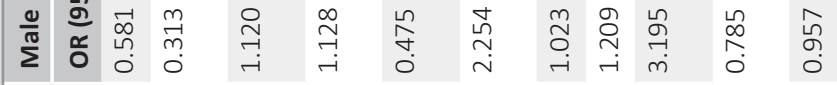

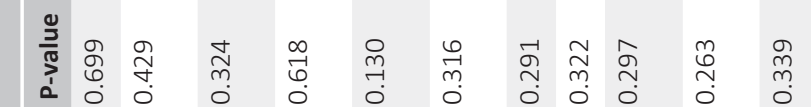

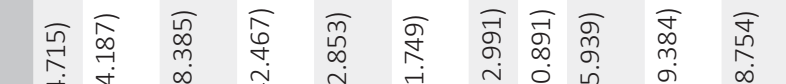

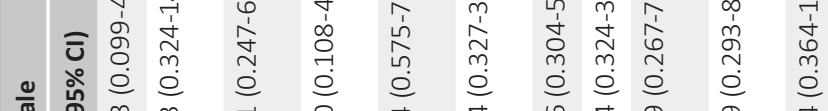

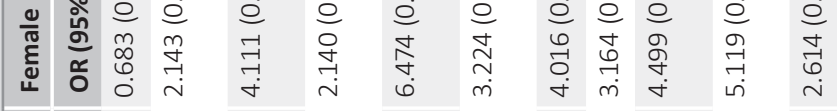

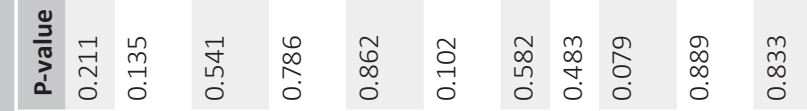

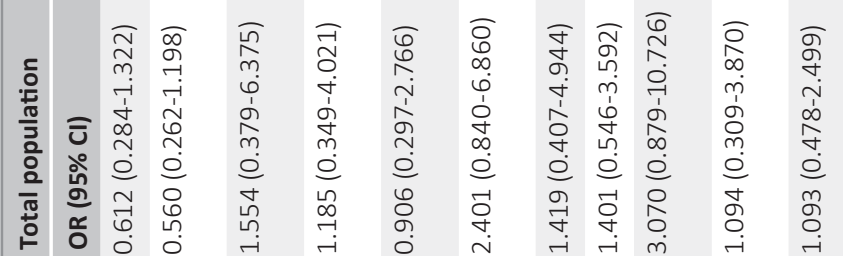

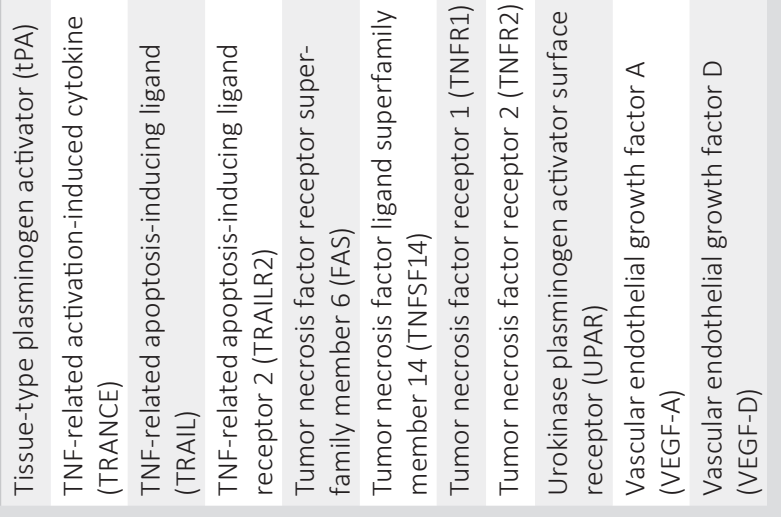



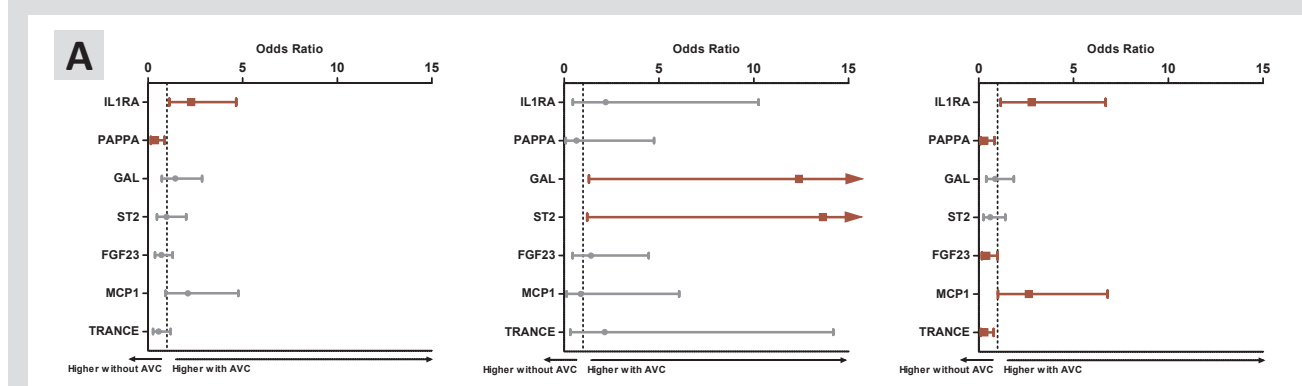

B
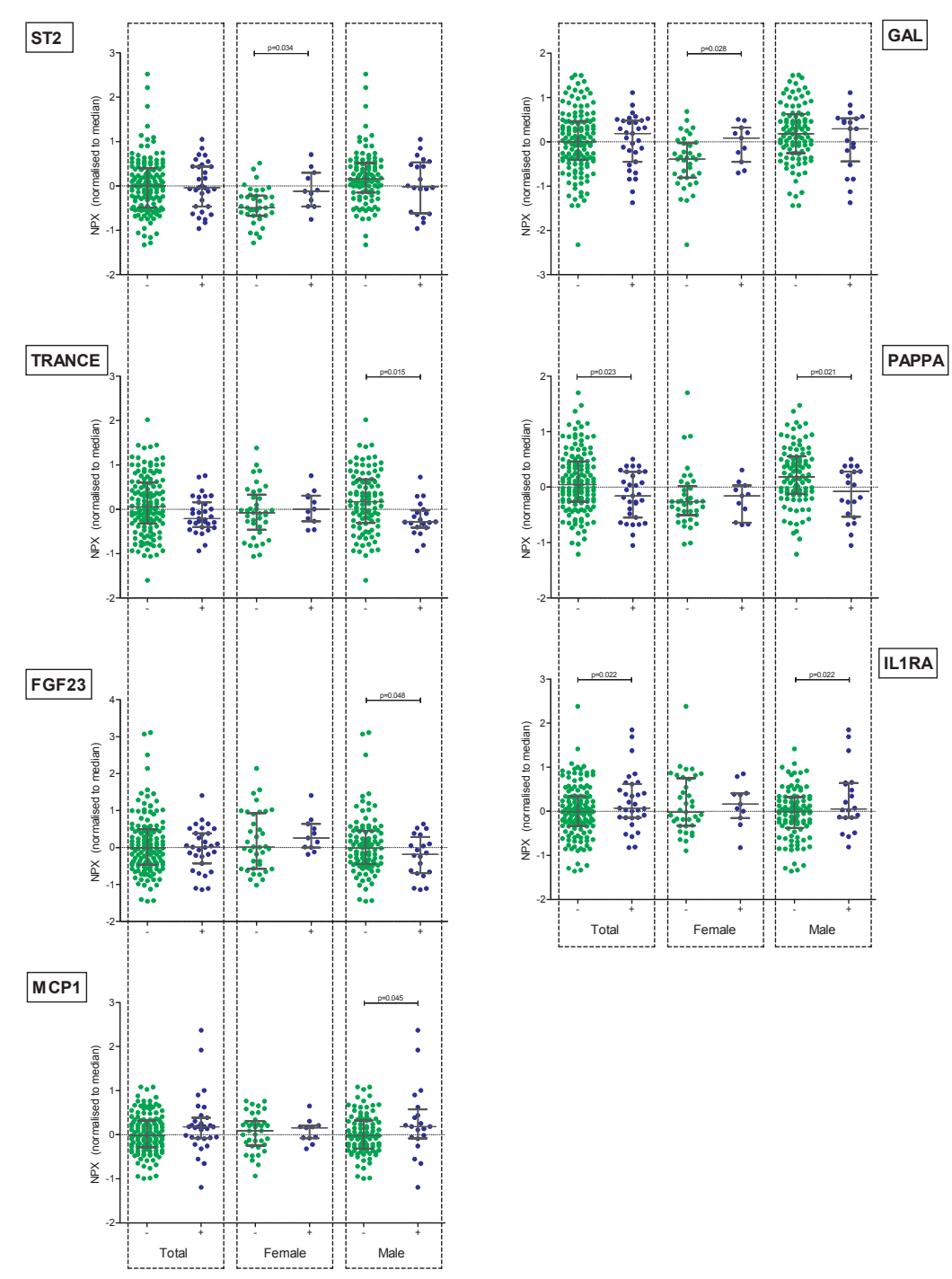

GAL

PAPPA
Figure 1. A: Odds ratios and $95 \%$ confidence intervals (corrected for age, sex and presence of AF) for biomarkers with increased or decreased odds for having AVC in the total population, female and male populations. Significant markers are displayed in red. Left panel: Total population, middle panel: female population, right panel: male population. B: Unadjusted boxplots of 7 biomarkers found to be significantly different between patients (male/female) with and without AVC. Median and interquartile ranges are shown. GAL: galanin peptides, MCP1: Monocyte chemotactic protein 1, PAPPA: Pappalysin-1, TRANCE: TNF-related activation induced cytokine, FGF23: Fibroblast growth factor 23, ST2: ST2-protein, IL1RA: Interleukin 1 receptor antagonist receptor

\section{DISCUSSION AND CONCLUSION}

This study shows that seven circulating biomarkers show subtle differential expression levels associated with AVC in very early stage aortic valve calcification. We found differential expressions of several biomarkers and these biomarkers are involved in all three processes relevant for aortic valve degeneration i.e. inflammation, fibrosis and calcification. ${ }^{2}$

Recently, it was proposed that in aortic valve disease, sex-specific mechanisms should be investigated in future studies. ${ }^{3}$ Women who develop severe aortic valve disease have a lower valvular calcium content when compared to men, suggesting a more dominant role for fibrosis in disease progression in women. The effects of estrogen and testosterone are thought to play a role in determination of the dominance of fibrosis in women versus the calcification dominance in men. ${ }^{3}$ In our study, we confirm a higher expression of ST2 (myocyte stress and fibrosis ${ }^{8-10}$ ) and galanin peptides (myocardial remodeling in response to stress ${ }^{11}$ ) in association with AVC in women. In men, aortic valve disease is dominated by calcification, and in the current study lower expression levels of TRANCE (or RANKL) were associated with AVC. Additionally, lower expression of pappalysin-1, involved in insulin-like growth factor-1 signaling and osteoblast differentiation of valvular interstitial cells (VICS) ${ }^{12}$ was associated with AVC in men. Also, lower expression of FGF23, involved in metabolic bone disease and vascular calcification, was associated with AVC in men. These data suggest that triggers for VIC phenotype change differ between phases of aortic valve disease progression. Moreover, circulating biomarkers involved in inflammation, oxidative stress and endothelial activation (IL1RA, MCP-1) 13,14 $^{14}$ showed, as expected, a higher expression. ${ }^{15}$

The results of the current study show new insights in biomarkers involved in aortic valve disease in a low risk population without significant risk factors for AVC. Therefore, our study adds valuable information to increase knowledge on the mechanisms of aortic valve disease. However, cautious interpretation is warranted. This retrospective, cross-sectional study with an explorative nature has a relatively small sample size, especially when stratified by sex. Therefore, we propose for the biomarker panel results to be confirmed in larger studies.

Genesis and progression of aortic valve disease is a complex process. We found a number of biomarkers involved in several processes associated with aortic valve disease. Single biomarkers clearly lack sensitivity to form the base for analyzing all processes involved at different stages (including the initiation phase) of the disease, given that these biomarkers might be derived from different sources within the body. Investigating panels of biomarkers in future studies can overcome this problem in addition to further development of imaging technologies to visualize the disease in its earliest/premature phases. Moreover, integration of (a combination of) specific biomarkers and imaging could more successfully assess the risk of rapid progression, which would facilitate patient counseling and help personalize follow up of patients. Ultimately, gaining knowledge in the processes involved in the genesis and the progression phases of aortic valve 
disease will provide us with opportunities to investigate potential therapeutic targets to slow/ reduce/regress aortic valve calcification and disease progression. With that, the opportunity to delay surgical interventions in patients with aortic valve disease might be imminent.

\section{REFERENCES}

CES HJ, Sng GK et al. Aortic stenosis, atherocalcification and inflammation? Eur Heart 1 2013:34:1567-74

2. Peeters F, Meex SJR, Dweck MR et al. Calcific aortic valve stenosis: hard disease in the heart: A biomolecular approach towards diagnosis and treatment. Eur Heart

Sritharen Y, Enriquez-Sarano M, Schaff HV, Casaclang-VerIs It Both Fibrocalcific and Sex Specific? Physiology (Bethesda) 2017;32:182-196.

4. Baumgartner H, Falk V, Bax JJ et al. 2017 ESC/EACTS Guidelines for the management of valvular heart disease. Eur Weiis $B$, Pisters $R$, Haest $R$ R et

Weijs B, Pisters R, Haest RJ et al. Patients originally diagfrom insidious coronary artery disease compared to healthy sinus rhythm controls. Heart Rhythm 2012;9:1923-9. 6. Assarsson E, Lundberg $\mathrm{M}$, Holmquist $\mathrm{G}$ et al. Homogenous 96-plex PEA immunoassay exhib high high sensitivity, specAggarwal SR, Clavel MA Messika-Zeitoun Detal. Sex differences in aortic valve calcification measured by multidetector computed tomography in aortic stenosis. Circ Cardiovasc Imaging 2013;6:40-7.

8. Chin CW, Djohan AH, Lang CC. The role of cardiac biochemical markers in aortic stenosis. Biomarkers 2016;21:316-27. of novel biomarkers of cardiovascular stress in patients with aortic stenosis undergoing valve replacement. Heart 2015;101:1382-8.

10. Shah RV, Januzzi IL, Jr. ST2: a novel remodeling biomarker in acute and chronic heart failure. Curr Heart Fail Rep

1. Timotin A, Pisarenko O, Sidorova M et al. Myocardial progalanin fragment. Oncotarget 2017:8:21241-21252.

12. Choi B, Lee S, Kim SM et al. Dipeptidyl Peptidase-4 Induces Aortic Valve Calcification by Inhibiting Insulin-Like Growt Factor-1 Signaling in Valvular Interstitial Cells. Circulation

3. Herder C, de Las Heras Gala T, Carstensen-Kirberg M et al. Risk of Cardiovascular Disease: Meta-Analysis of Six Population-Based Cohorts. Arterioscler Thromb Vasc Bio 2017;37:1222-1227.

14. Deshmane SL, Kremlev S, Amini S, Sawaya BE. Monocyte chemoattractant protein-1 (MCP-1): an overview. J Inte-

15. Aikawa $E$, Nahrendorf M, Sosnovik $D$ et al. Multimodality activities in early aortic valve disease Circulation 2007;115:377-86 


\section{SUPPLEMENTAL MATERIAL}




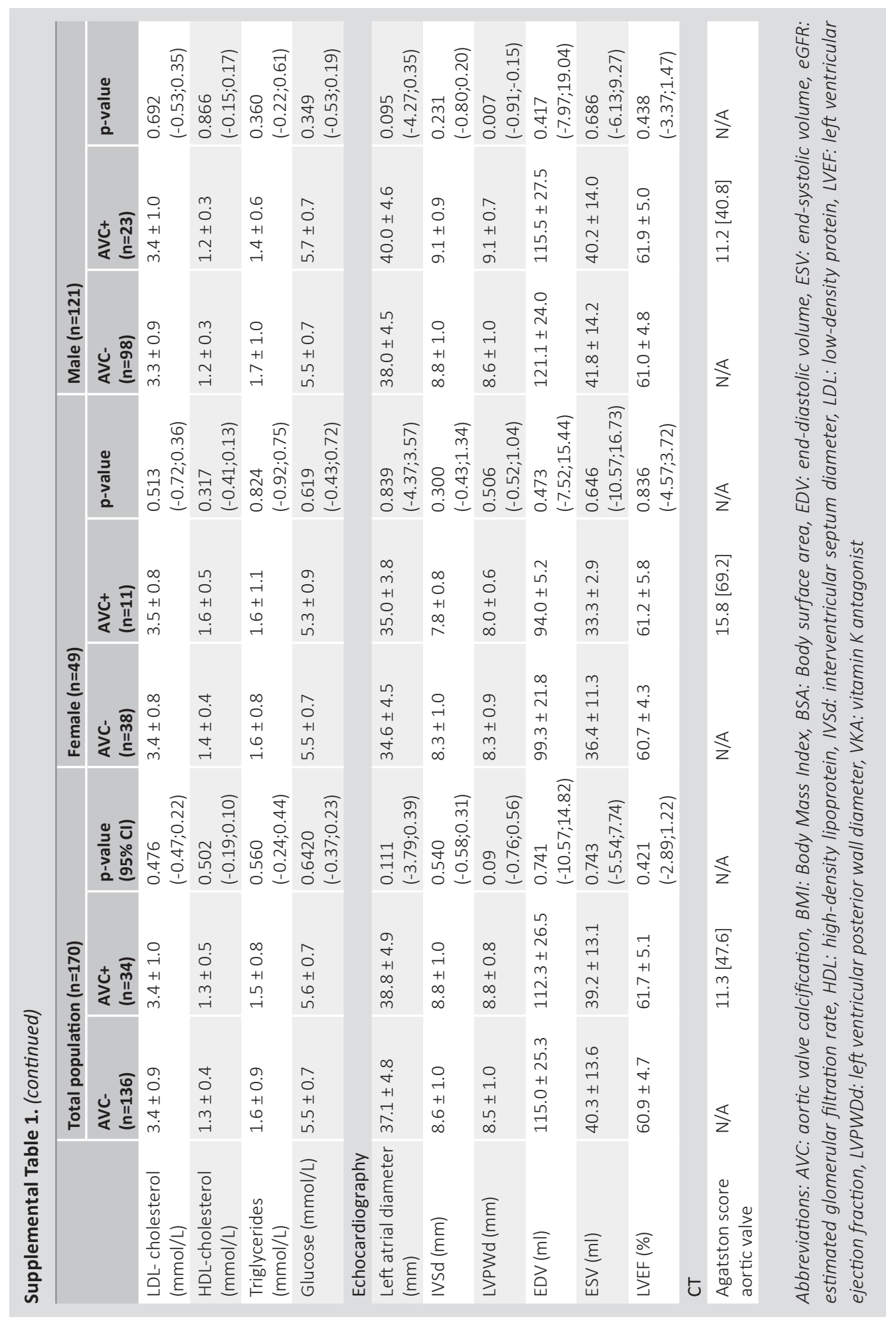




\section{Biological variation of cardiac markers in patients with aortic valve stenosis}

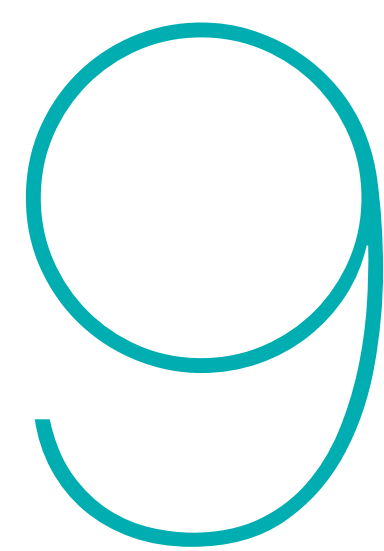

Frederique E.C.M. Peeters ${ }^{1}$, Bas L.J.H. Kietselaer ${ }^{2}$, Judith M. Hilderink ${ }^{3}$, Noreen van der Linden ${ }^{3}$, Marijke Niens ${ }^{4}$, Harry J.G.M. Crijns ${ }^{1}$, Steven J.R. Meex ${ }^{3}$

${ }^{1}$ Maastricht University Medical Center+ and CARIM, department of Cardiology, Maastricht, the Netherlands,

2 Zuyderland Medical Centre, Department of Cardiology, Heerlen, the Netherlands,

${ }^{3}$ Maastricht University Medical Center+ and CARIM, department of Clinical Chemistry,

Maastricht, the Netherlands,

${ }^{4}$ Laurentius ziekenhuis, department of Clinical Chemistry, Roermond, the Netherlands. 


\section{ABSTRACT}

Background: Cardiac biomarkers hold promise for the follow-up and management of aortic valve stenosis. When interpreting serial biomarker measurements of patients with aortic valve stenosis (AS) it can be challenging to distinguish "real changes" from "random fluctuation". Hence, robus estimation of the biological variation of these biomarkers is essential. In the present study we assessed biological variation of BNP, NT-proBNP, troponin-T, troponin-I and ST2 in subjects with stable AS.

Methods: Serial blood sampling was performed in 25 subjects with moderate AS- confirmed by echocardiography- and all free from acute cardiovascular events in the past 6 months. Blood samples were taken on 7 standardized occasions during 1 year. Analytical variation $\left(\mathrm{CV}_{A}\right)$, within-subject biological variation $\left(\mathrm{CV}_{1}\right)$, between-subject biological variation $\left(\mathrm{CV}_{\mathrm{G}}\right)$, index of individuality (II) and reference change values (RCV) were calculated for all cardiac biomarkers

Results: Within-subject biological variation was highest for BNP $(62.0 \%, 95 \% \mathrm{Cl} 52.5 ; 75.4)$ and lowest for troponin-I $(9.2 \%, 95 \% \mathrm{Cl} 2.8 ; 13.8)$. Between-subject biological variation exceeded the $\mathrm{CV}_{1}$ for all biomarkers except BNP, and ranged from $19.8 \%(95 \% \mathrm{Cl} 13.8 ; 33.4)$ for ST2 to $57.2 \%$ 95\%Cl 40.4;97.3) for troponin-T. NT-proBNP, troponin-T and ST2 revealed analytical variation $<5 \%$, whilst BNP and troponin-I showed a higher CV, (19.7 and 14.9 respectively). All biomarkers except BNP showed marked individuality, with II ranging from 0.21 to 0.67 (BNP 1.34).

Conclusion: This study provides the first biological variation estimates of cardiac biomarkers in patients with stable AS. These estimates allow a more evidence-based interpretation of biomarke changes in the follow up and management of patients with AS.

Key words: Aortic valvestenosis, progression, cardiac biomarkers, biologicalvariation, echocardiography 
and was approved by the local Institutional Review Board. It was registered at www.clinicaltrials.gov as NCT02510482. All study subjects provided written informed consent.

\section{Study design}

All subjects visited the outpatient clinics of Cardiology of our center on 7 occasions during 1 year (baseline and 1 day, 1 week, 1 month, 3 months, 6 months and 12 months). Visits and blood samplings were performed in a standardized manner and all patients were asked to refrain from intense physical labor and exercise training 2 days before each visit. All patient visits took place between 08.00 and $09.00 \mathrm{AM}$, during which standard history taking and a standardized questionnaire (including medication use) were performed. Blood sampling was performed through standard venipuncture in seated position.

\section{Laboratory measurements}

Blood samples were collected in serum and ethylenediaminetraacetic acid (EDTA) tubes. Immediately after collection, standard hematological parameters (hemoglobin, hematocrit, white blood cells, neutrophils) were measured in EDTA-samples using the Sysmex XE-5000 analyzer (Sysmex Corporation, Kobe, Japan). The serum samples were allowed to clot and were centrifuged after 25 minutes (12 $\mathrm{min}, 2500 \mathrm{~g}$ ). Directly after aliquoting, samples were stored at $-80^{\circ} \mathrm{C}$ until further analyses.

NT-proBNP and high-sensitivity troponin-T levels were measured on the COBAS 6000 analyzer. High-sensitivity cardiac troponin-I was measured with the STAT high-sensitivity troponin I assay (Architect 2000, Abbott Diagnostics). BNP was measured on the Architect analyzer (Abbott Diagnostics) and ST2 using the Presage ${ }^{\circledR}$ ST2 Assay (Critical Diagnostics). To estimate analytical variation, $60-100 \%$ of BNP, NT-proBNP, troponin-T, troponin-I and ST2 measurements were performed in duplicate.

\section{Echocardiography}

Standard two-dimensional transthoracic echocardiography was performed by an independen observer prior to inclusion and during regular visits to the outpatient clinics according to the European Association of Echocardiography (EAE) guidelines. ${ }^{24}$

\section{Statistical analyses}

Cochran's $\mathrm{C}$ test was used to test data for homogeneity in analytical and within-subject biologica variances as suggested by Fraser and Harris. ${ }^{25}$ Subjects were excluded until homogeneity of variances was achieved. Between-subject outliers were identified using the criteria of Reed.11,26,27 Between-subject $\left(\mathrm{CV}_{G}\right)$ and within-subject biological variation $\left(\mathrm{CV}_{1}\right)$ and analytical variation $\left(\mathrm{CV}_{A}\right)$ were calculated using a balanced analysis of variance with a nested random design in two levels. ${ }^{28}$ The method of Burdick and Graybill was used accordingly to calculate $95 \%$ confidence intervals (Cl) of the variance components. ${ }^{28,29}$ The index of individuality (II) and Reference Change Value (RCV) were calculated according to the method described by Petersen et al. and Fraser and Harris. $25,30,3$ II was calculated using the formula: $\|=V\left(C_{1}^{2}+\left(\mathrm{CV}_{A}^{2}\right) / C V_{G}\right.$. The RCV was calculated using the formula: $R C V=Z^{*} V\left(2^{*}\left(C V_{1}{ }^{2}+C V_{d}^{2}\right)\right)$. In this formula, $Z$ represents the number of standard deviations appropriate for the desired level of statistical significance for a bidirectional change. For RCV calculations in this study, a Z-score of 1.96 was used. Additionally, RCVs were calculated and evaluated after log-normal transformation. ${ }^{32}$ All statistical analyses were performed using SPSS statistics version 22 (IBM Corp, Armonk, NY).

\section{RESULTS}

\section{Baseline population characteristics}

A total of 25 subjects with moderate aortic valve stenosis participated in the current study. Mean age $( \pm S D)$ was $66 \pm 6$ years, and $44 \%(n=11)$ subjects were female. All subjects had moderate aortic valve stenosis on baseline echocardiography (median [IQR] mean gradient 25 [11] $\mathrm{mmHg}$ maximum velocity $340[65] \mathrm{cm} / \mathrm{s}$ and aortic valve area $1.3[0.2] \mathrm{cm}^{2}$ ) and median [IQR] left ventricular ejection fraction (LVEF) 63 [5]\%. None had complaints attributable to aortic valve stenosis. Baseline concentrations of all biomarkers are shown in figure 1.

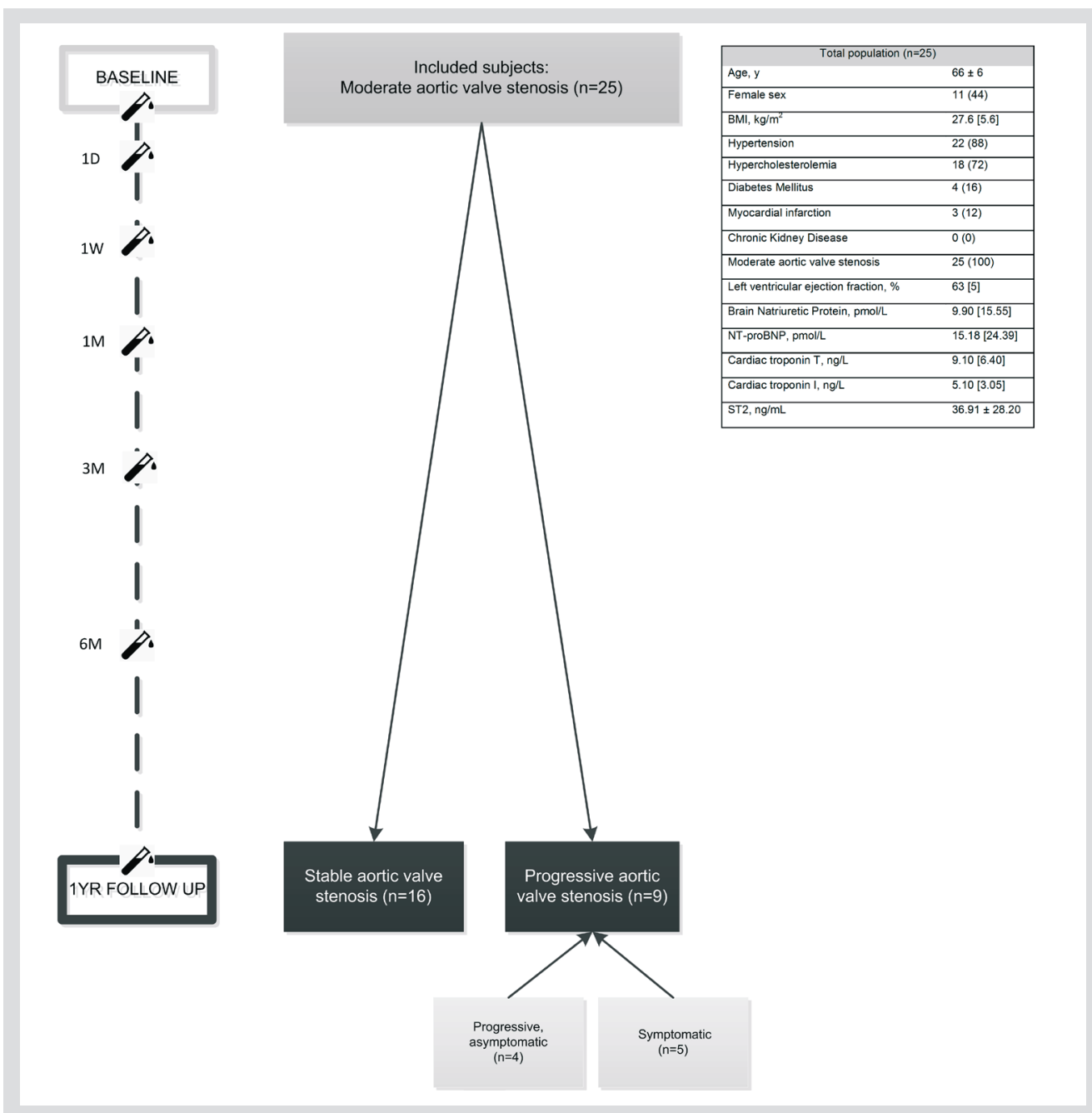

Figure 1. Flowchart of the population: including baseline characteristics and follow-up

Continuous variables are expressed as mean $\pm S D$ or median [IQR] depending on their distribution. Categorical variables are reported as $n(\%)$. 


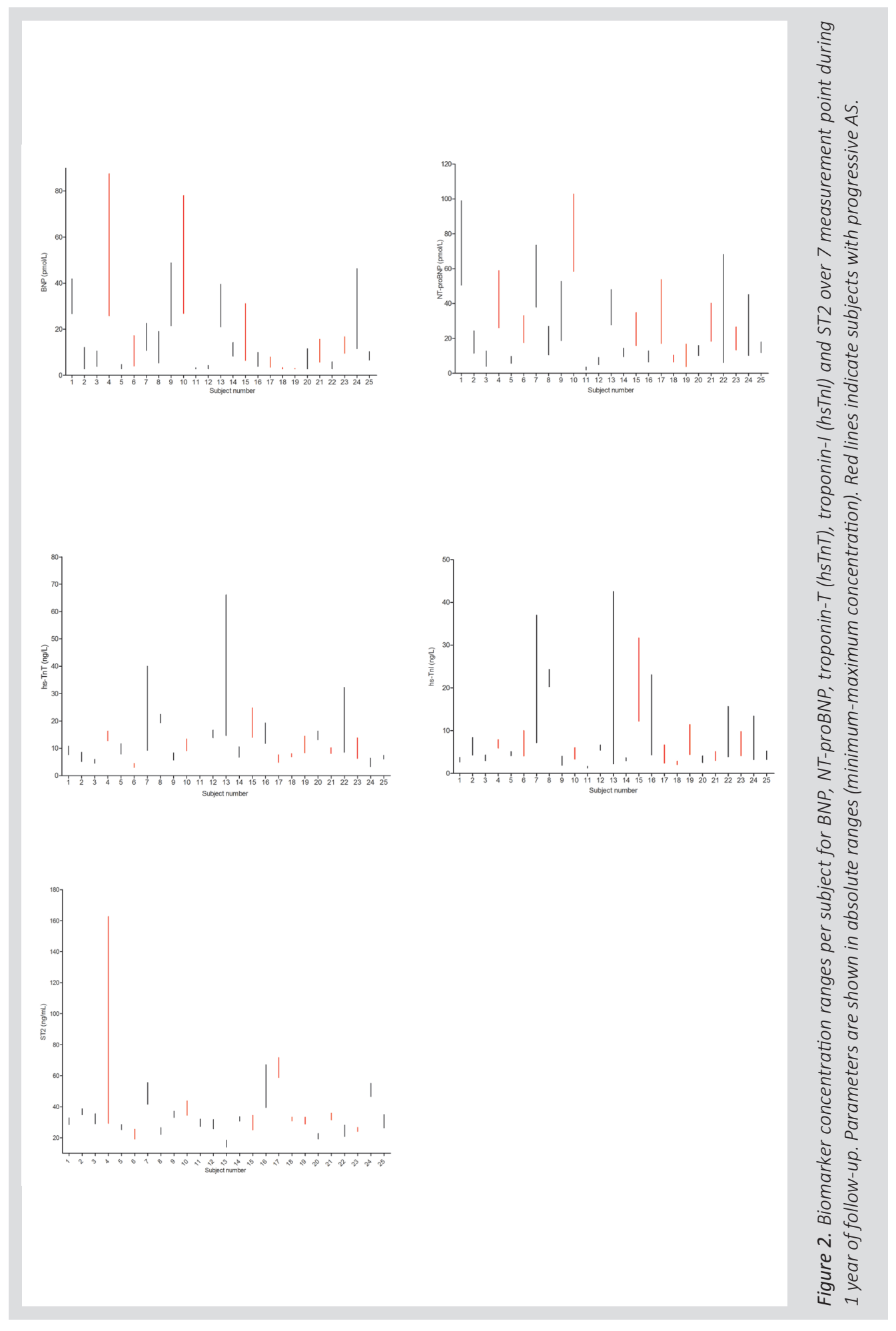

\section{Biological variation in stable aortic valve stenosis}

Sample collection was complete for all subjects. Figure 1 shows the baseline characteristics of the total population. Biomarker concentration ranges per subject (BNP, NT-proBNP, troponin-T, troponin-I and ST2) are plotted in Figure 2.

In 9 subjects, aortic valve stenosis was progressive (Figure 1). Therefore, the primary analysis to determine biological variation was performed in the group of subjects that remained clinically stable during follow-up ( $n=16$ ). An overview of outliers and excluded subjects per biomarker is provided in Supplemental Table 1. Variation components of all cardiac biomarkers are listed in Table 1.

NT-proBNP, troponin-T, troponin-I and ST2 revealed lower within-subject (CV) than betweensubject $\left(\mathrm{CV}_{\mathrm{G}}\right)$ values. Troponin-I demonstrated the lowest and BNP the highest $\mathrm{CV},(9.1 \%, 95 \% \mathrm{C}$ 2.8;13.8 versus $62.0 \%, 95 \% \mathrm{Cl} 52.5 ; 75.4)$. Except for $\mathrm{BNP} \mathrm{CV}_{\mathrm{G}}$ was consistently higher than $\mathrm{CV}_{1}$, and ranged from $19.8 \%(95 \% \mathrm{Cl} 13.8 ; 33.4)$ for ST2 to $57.2 \%$ (95\% Cl 40.4;97.3) for troponin-T. Duplicate measurements allowed calculation of analytical variation: NT-proBNP, troponin-T and ST2 were measured with a $\mathrm{CV}_{\mathrm{A}}<5 \%$, whereas BNP and troponin-I showed higher analytical variation (19.7\% and $14.9 \%$, respectively).

Additionally, the variation between specific set points, known as the index of individuality (II) was calculated. All biomarkers except BNP showed marked individuality, with II ranging from 0.2 to 0.7 (BNP 1.3). High individuality of biomarkers (II<0.6) implies that the use of reference change values for monitoring offers substantial benefit over classical population based reference intervals.

Table 1. Analytical variation, biological variation, indexes of individuality and reference change values in stable aortic valve stenosis

\begin{tabular}{|c|c|c|c|c|c|c|c|}
\hline & \multirow[t]{2}{*}{ Mean concentration } & \multicolumn{3}{|c|}{ Variance components } & \multicolumn{2}{|l|}{$\mathrm{RCV}^{\mathrm{b}}$} & \multirow[t]{2}{*}{ II } \\
\hline & & $\mathrm{Cv}_{\mathrm{G}}$ & $\mathrm{CV}_{1}$ & $\mathrm{CV}_{\mathrm{A}}{ }^{\mathrm{a}}$ & Normal & Log-normal & \\
\hline BNP & $5.7 \mathrm{pmol} / \mathrm{L}$ & $\begin{array}{l}48.4 \\
(28.6 ; 91.7)\end{array}$ & $\begin{array}{l}62.0 \\
(52.5 ; 75.4)\end{array}$ & $\begin{array}{l}19.7 \\
(17.1 ; 23.4)\end{array}$ & * & $52.6 ;-34.5$ & 1.3 \\
\hline NT-proBNP & $9.3 \mathrm{pmol} / \mathrm{L}$ & $\begin{array}{l}43.0 \\
(27.7 ; 88.8)\end{array}$ & $\begin{array}{l}22.0 \\
(18.3 ; 27.5)\end{array}$ & $\begin{array}{l}1.9 \\
(1.6 ; 2.3)\end{array}$ & * & $44.6 ;-30.9$ & 0.5 \\
\hline Hs-TnT & $9.2 \mathrm{ng} / \mathrm{L}$ & $\begin{array}{l}57.2 \\
(40.4 ; 97.3)\end{array}$ & $\begin{array}{l}11.2 \\
(9.6 ; 13.5)\end{array}$ & $\begin{array}{l}3.7 \\
(3.2 ; 4.3)\end{array}$ & * & $43.1 ;-30.1$ & 0.2 \\
\hline $\mathrm{Hs}-\mathrm{Tnl}$ & $3.6 \mathrm{ng} / \mathrm{L}$ & $\begin{array}{l}35.0 \\
(23.3 ; 67.6)\end{array}$ & $\begin{array}{l}9.2 \\
(2.8 ; 13.8)\end{array}$ & $\begin{array}{l}14.9 \\
(12.7 ; 18.0)\end{array}$ & * & $73.1 ;-42.2$ & 0.5 \\
\hline ST2 & $28.4 \mathrm{ng} / \mathrm{mL}$ & $\begin{array}{l}19.8 \\
(13.8 ; 33.4)\end{array}$ & $\begin{array}{l}13.1 \\
(11.3 ; 15.6)\end{array}$ & $\begin{array}{l}2.0 \\
(1.7 ; 2.4)\end{array}$ & 36.83 & ** & 0.7 \\
\hline
\end{tabular}

Values are \% (95\% Cl); $\mathrm{Cl}=$ confidence interval; $C V_{A}=$ analytical coefficient of variation; $C V_{G}=$ between person coefficient of variation; $C V_{1}=$ within-person biological coefficient of variation; $R C V=$ reference change value

BNP: brain natriuretic peptide; NT-proBNP: N-terminus pro-brain natriuretic peptide; $h$-TnT: high-sensitivity troponin T; hs-Tnl: high-sensitivity troponin I; II: index of individuality

${ }^{a}$ On the basis of duplicate measurements, ${ }^{b}$ On the basis of a z-score of 1.96

*Non-normal distribution; **Normal distribution 


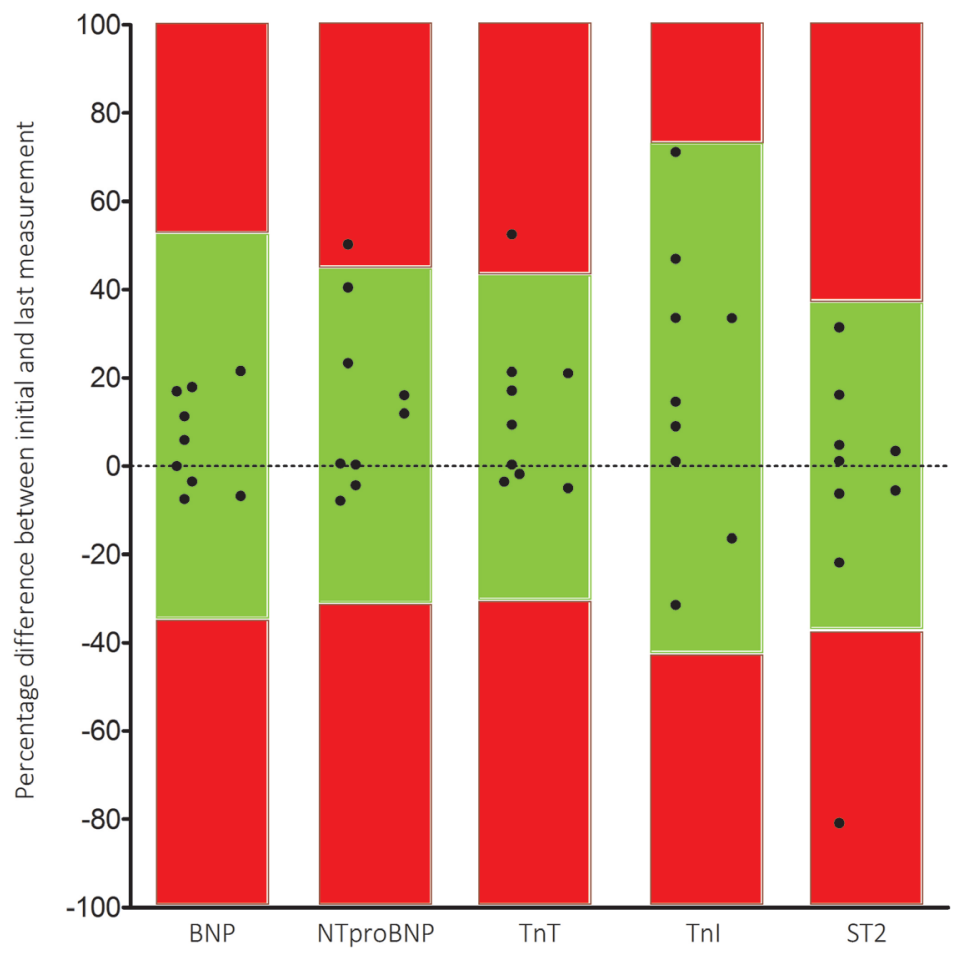

Figure 3. Observed variation in 1 year of patients with progressive AS.

Black dots represent the difference between initial and last measurement (1 year). Green boxes represent (log-normal) reference change values and dots within these boxes represent random fluctuations. Dots in the red boxes are considered true changes (outside the RCV limits). BNP and NT-proBNP: brain natriuretic peptides, TnT: troponin-T, Tnl: troponin-I.

\section{Biomarkers in stable and progressive aortic valve stenosis}

The subpopulation of subjects showing progressive aortic valve disease $(n=9)$ was explored. Five patients progressed to symptomatic aortic valve stenosis after their 6 months visit. Another 4 remained asymptomatic, but showed progressive disease during echocardiographic examination after 1 year of follow up. Since biomarker results from these progressive patients cannot be used to determine biological variation, we explored whether the investigated biomarkers have potentia value to discriminate stable from progressive AS. The annual variation (defined as the difference between initial measurement and measurement after 1 year) was calculated for each biomarker in all progressive subjects. These values were compared to the (log-normal) RCVs found in the stable population (Figure 3). Troponin-T and NT-proBNP variation was higher in 1 of 9 subjects with progressive disease, whereas the RCV of ST2 was surpassed in 1 of 9 subjects. None of the progressive subjects showed variation higher than the RCV for BNP and troponin-I.
DISCUSSION

The management and follow up of patients with aortic valve stenosis would benefit from cardiac biomarker changes that reflect or even precede clinical progression of aortic valve stenosis. $7,8,33-36$ The interpretation whether an observed biomarker change over time is clinically relevant is challenging. To interpret serial measurements, knowledge about variation components of biomarkers is essential.

This study is the first to examine biological variation of several cardiac biomarkers (BNP NT-proBNP, troponin-T, troponin-I and ST2) in subjects with stable moderate aortic valve stenosis. We report two major findings: First, we found substantial biological variation within and between subjects with $A S\left(C V_{1}=9-62 \%\right.$ and $\left.C V_{G}=20-58 \%\right)$, corresponding with previous studies that were conducted in healthy subjects. Within-subject variability was relatively small for ST2 and troponin-T and -1 , whereas within-subject variation was high for BNP and NT-proBNP. These results show that observed changes in consecutively measured BNP and NT-proBNP samples must be relatively large to meet the threshold for a "true" change, while smaller changes between serial measurements of troponin-T and ST2 are indicative of a significant change. Studies examining biological variation have been performed in healthy populations, but a growing interest in variation components of biomarkers in populations with (cardiovascular) disease resulted in newer studies addressing biological variation in heart failure and chronic kidney disease. 15,17-20,37 We found that indices of biological variation in stable aortic valve stenosis approximated indices found in studies investigating biological variation in healthy subjects and chronic and stable heart failure. ${ }^{17,20,37}$

Second, between-subject variation was higher than within-subject variation in all biomarkers but BNP in our population. Both affect the index of individuality and therefore, we found low indexes of individuality in all biomarkers (except BNP) and thus marked individuality in our population. Therefore, the use of population based reference values is of limited utility. $13,14,3$ Instead, the use of reference change values (RCV) is of value in these biomarkers.

From a clinical perspective, a low index of individuality underlines the importance to use of reference change values instead of general population-based reference intervals to interpret serial measurements in an individual. However, the individual variation is undervalued in daily practice, as we tend to interpret biomarkers above or below general thresholds to identify a patient with low or high risk. The use of RCVs with serial biomarker measurements bears potential to integrate in the development of tailored treatment strategies in personalized medicine.

\section{Study limitations}

Potential limitations of the current study merit attention. First, we included 25 patients with stable moderate aortic valve stenosis at baseline for analysis of biological variation. After 1 year of follow up, 9 patients showed progressive disease. Sixteen patients with stable aortic valve stenosis were left for primary analysis, providing us with sufficient power to make reliable estimations for variation components for primary analyses. ${ }^{28}$ Second, the size of our population did not allow stratification in sex- or- age-groups. Further exploration of the role of these biomarkers in personalized clinical decision-making would be interesting.

\section{CONCLUSION}

This study provides the first biological variation estimates of BNP, NT-proBNP, troponin-T, troponinand ST2 in patients with stable aortic valve stenosis. These estimates allow a more evidence-based interpretation of biomarker changes in the follow up and management of patients with aortic valve stenosis. 
REFERENCES

Toutouzas K, Stathogiannis K, Latsios G et al. Biomarkers in 20. Wu AH, Wians F, Jaffe A. Biological variation of galectin-3 aortic valve stenosis and their clinical significance in tran-
scatheter aortic valve implantation. Curr Med Chem 2017.

2. van Holten TC, Waanders LF, de Groot PG et al. Circulating 21. Bartlett WA, Braga F, Carobene A et al. A checklist for critibiomarkers for predicting cardiovascular disease risk; a sys- cal appraisal of studies of biological variation. Clinical tematic review and comprehensive overview of meta-anal- 22 chemistry and laboratory medicine 2015;53:879-85.

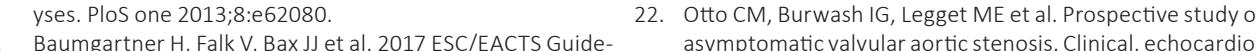
lines for the management of valyular heart disease. Euro- graphic, and exercise predictors of outcome. Circulation pean heart journal 2017;38:2739-2791. 1997;95:2262-70.

4. Nishimura RA, Otto CM, Bonow RO et al. 2014 AHA/ACC 23. Rosenhek R, Binder T, Porenta $G$ et al. Predictors of outGuideline for the Management of Patients With Valvular come in severe, asymptomatic aortic stenosis. N EnglJ Med Heart Disease: a report of the American College of Cardiorogy/American Heart Association Task Force on Practice 24. Evangelista A, Flachskampf F, Lancellotti P et al. European Peeters F, Meex SIR, Dweck MR et al. Calcific aortic valve ste- standardization of performance, digital storage and report nosis: hard disease in the heart: A biomolecular approach ing of echocardiographic studies. Eur I Echocardiogr towards diagnosis and treatment. European heart journal 2017. Small A, Kiss D, Giri J et al. Biomarkers of Calcific Aortic 25. Fraser CG, Harris EK. Generation and application of data on Valve Disease. Arterioscler Thromb Vasc Biol 2017,37.623-632. biological variation in clinical chemistry. Crit Rev Clin Lab aortic stenosis. European heart journal 2014:35:2312-21.

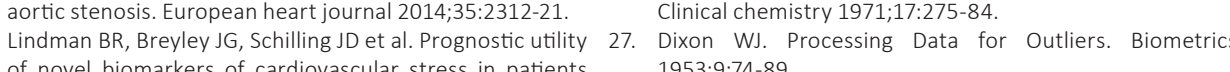
of novel biomarkers of cardiovascular stress in patients with aortic stenosis undergoing valve replacement. Heart 28 Dahou A, Clavel MA,

Septide and MA, Capoulade R et al. B-Type Natriuretic Stratification in Low-Flow, Low-Gradient Aortic Stenosis: Substudy of the TOPAS Study. JACC Cardiovascular imaging 2018;11:939-947.

10. Dweck MR, Everett RJ. Multibiomarker Strategies in Aortic 3 Fraser $C G$. Biological variation f from principles to practice

Washington DC: AACC Press, 2001.

12. Bailey D, Bevilacqua V, Colantonio DA et al. Pediatric within-day biological variation and quality specification for 38 biochemical markers in the CALIPER cohort. Clinical chemistry 2014;60:518-29,

13. Fraser CG. Reference change values. Clinical chemistry and

interindividual variation on 3 1974;20:1535-42.

15. Frankenstein L, Wu AH, Hallermayer K, Wians FH, Jr., Giannitsis $E$, Katus $H A$. Biological variation and reference viduals during short and intermediate follow-up periods. Clinical chemistry 2011;57:1068-71.

16. Klinkenberg LJ, van Dijk JW, Tan FE, van Loon LJ, van Diejen-Visser MP, Meex SJ. Circulating cardiac troponin exhibits a diurnal hyythm.J Am Coll Cardiol 2014;63:1788-95. of biomarkersin patients with chron Kobold fall al. ariability controls. European journal of heart failure 2017:19:357-365.

van der Linden N Hilderink IM, Cornelis T et al. TwentyFour-Hour Biological Variation Profiles of Cardiac Troponin in Individuals with or without Chronic Kidney Disease. Clinical chemistry 2017,63:1655-1656.

9. Wu AH, Smith A. Blological varinton of the natriuretic pep-e method used on the resulting estimate of normat range. 1953;9:74-89.

and S Tetersen PH, Sandberg S. Confidence interval and power calculations for within-person biological varianumber of samples, and number of individuals. Clinica chemistry 2012;58:1306-13.

Burdick RK, Graybill FA. Confidence intervals on variance components. New York: Marcel Dekker, 1992.

che Clinical chemistry 1983;29:25-30

31. Petersen PH Fraser $C G$ Sandherg $S$, Goldschmidt $H$. The index of individuality is often a misinterpreted quantity characteristic. Clinical chemistry and laboratory medicin 1999;37:655-61.

. Fokkema MR, Hermann Z, Muskiet FA, Moecks J. Refer-

政 Natriuretic Peptide Changes for Predicting Outcome in (

Sancellotti P, Dulgheru R, Magne J et al. Elevated Plasma Outcome in Aortic Stenosis. Plos one 2015:10 Rosjo H. Andreassen J, Edvardsen T, Omland T. Prognostic usefuness of circulating high-sensitivity troponin Tin aortic stenosis and relation to echocardiographic indexes of cardiac function and anatomy. The American journal of cardiology 2011;108:88-91. tissue and a a past, present and future. Current opinion in cardiolog 2018;33:125-133.

7. Schindler El, Szymanski JJ, Hock KG, Geltman EM, Scott MG. Short-and Long-term Biologic Variability of Galectin-3 and Other Cardiac Blomarkers in Patients with Stable Heart FallHarris EK. Statisticas aspects of reference values in pathology Progress in clinical pathology 1981:8:45-66. 


\section{SUPPLEMENTAL MATERIAL}




\section{Clinical and echocardiographic determinants in bicuspid aortic dilatation: results from a longitudinal observational study}

Frederique E.C.M. Peeters ${ }^{1}$, Noreen Van der Linden ${ }^{2}$, Alissa L.L. Thomassen ${ }^{1}$ Harry J.G.M. Crijns ${ }^{1}$, Steven J.R. Meex², Bas L.J.H. Kietselaer ${ }^{3}$

${ }^{1}$ Maastricht University Medical Center+ and CARIM, department of Cardiology, Maastricht, the Netherlands,

2 Maastricht University Medical Center+ and CARIM, department of Clinical Chemistry, Maastricht, the Netherlands,

${ }^{3}$ Maastricht University Medical Center+ and CARIM, department of Cardiology and Radiology, Maastricht, the Netherlands 


\section{ABSTRACT}

Background: Bicuspid aortic valve (BAV) disease is associated with aortic dilatation. Timing of follow-up and surgery is challenging. Hence, there is an unmet clinical need for additional risk stratification. It is unclear whether valve morphology is associated with dilatation rates. Therefore, the objective of this study was to examine the association between clinical and echocardiographic determinants (including valve morphology) and aortic dimension and the progression rate of dilatation.

Methods: Serial echocardiographic assessments of aortic dimensions between 1999 and 2014 in a population of 392 patients with bicuspid aortic valves in a tertiary care center in the Netherlands. Analyses using mixed linear models were performed.

Results: Mean age of participants was $48 \pm 17$ years and $69 \%$ were male. BAV morphology was associated with aortic dimensions, as well as age, sex, BSA and valvular dysfunction. Tubular ascending aorta, sinus of Valsalva and sinotubular junction showed a dilatation rate of $0.32 \mathrm{~mm} /$ year, $0.18 \mathrm{~mm} /$ year and $0.06 \mathrm{~mm} /$ year, respectively. Dilatation rate was not associated with valve morphology.

Conclusion: In the present study, there is no association between BAV morphology and aortic dilatation rates. Therefore, morphology is of limited use in prediction of aortic growth. Discovering fast progressors remains challenging.

Key words: bicuspid aortic valve, thoracic aorta, aortic dilatation, aortic dimensions, aortic dilatation rate

\section{INTRODUCTION}

The bicuspid aortic valve (BAV) is the most common congenital cardiac abnormality with an estimated prevalence of 13 per 1000 births in the general population. It is known for its heterogeneous presentation and its association with valvular and vascular complications, including aortic dilatation. Because of the association with aortic dilatation, BAV is considered as an aortopathy rather than a stand-alone valvulopathy. Yet, the natural course of dilatation varies widely, from virtually non-progressive to rapidly progressive, potentially leading to life-threatening aortic complications. ${ }^{2-4}$ Indication and timing of elective aortic surgical intervention remains challenging at present, as curren guidelines recommend variable treatment options based on studies advocating aggressive repair versus a conservative treatment approach. ${ }^{.-10}$

The exact pathophysiologic mechanisms underlying aortic dilatation in bicuspid aortopathy are not fully elucidated.11,12 Two mechanisms are proposed: firstly, the inherited or intrinsic predisposition. Several studies show abnormalities in the matrix, fibrillin and elastin fragmentation leading to accelerated degeneration of the media. ${ }^{13,14}$ Secondly, the hemodynamic consequences of BAV on aortic tissue by abnormal mechanical (local) stress (overload). ${ }^{15,16}$ Also, BAV morphology and its effect on blood flow in the ascending aorta has been studied as a potential contributing factor for development of aortic complications. Contradictory results exist concerning the possible association of valve morphology and both aortic dilatation and valvular function..$^{17,18}$

Optimizing the risk stratification of aortic dilatation in BAV patients is desirable, as this could impact timing of clinical follow-up and surgery. Few studies are available regarding dilatation rates and associated risk factors, showing variable outcomes, 2,19-21 Therefore, the aim of this study was to analyze the dimensions and dilatation rates of different segments of the ascending aorta and its determinants/ risk factors, including BAV morphology.

\section{METHODS}

Patients were identified in a tertiary care center in the Netherlands (Maastricht University Medical Centre, MUMC), by using the electronic database of all echocardiographic records from 1999 to 2014 Eligible patients were at least 18 years old and had a visually confirmed BAV on echocardiographic images. Serial echocardiographic images had to be available, at least six months apart. Patients with prior valve replacement surgery or surgery of the ascending aorta were excluded, whereas all degrees of valvular dysfunction were accepted. Clinical information was obtained through review of the available electronic hospital charts. This study was approved by the local Institutional Review Board and Ethics Committee.

\section{Echocardiography}

Measurements were performed in serial transthoracic echocardiographic images of eligible patients by two observers, using a dedicated workstation (Philips Xcelera software Version R3, Philips medica Systems, Best, the Netherlands). Presence of a bicuspid aortic valve was confirmed in a short-axis view and valve morphology was determined. In case of ambiguity, consensus was reached in the presence of a third observer. Bicuspid aortic valves were systematically classified during systole, according to Sievers classification, ${ }^{22}$ firstly as a raphe-related type 0 (bicuspid aortic valve without raphe), type 1 (bicuspid aortic valve with presence of one raphe) or type 2 (bicuspid aortic valve with presence of two raphes). Secondly, the exact fusion type was reported by description of the fusion patterns between the right coronary cusp (RCC), left coronary cusp (LCC) and non-coronary cusp (NCC).

Echocardiographic Doppler methods were used to assess the function of the aortic valve, in accordance with the American Society of Echocardiography (ASE) and American College of Cardiology/ American Heart Association (ACC/AHA) guidelines.., 23 


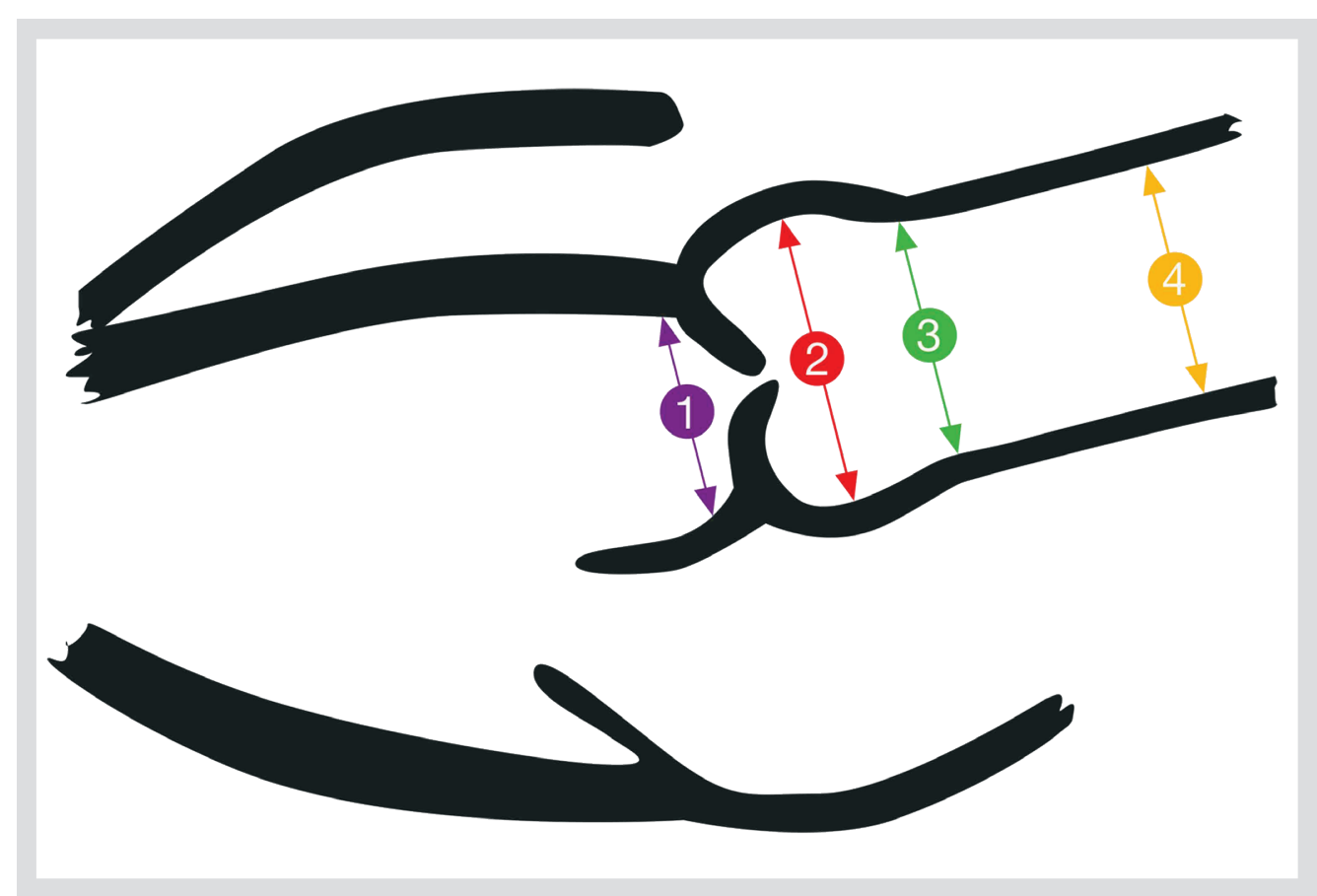

Figure 1. Schematic overview of measurement of aortic dimensions. 1: LVOT, 2: sinus of Valsalva, 3: sinotubular junction and 4: ascending aorta

Diameters of the left ventricular outflow tract (LVOT), sinus of Valsalva, sinotubular junction (STJ) and the tubular ascending aorta (TA) were measured from inner edge to inner edge in a parasterna long axis view. The LVOT diameter was assessed underneath the hinge points of the leaflets of the aortic valve, the sinus of Valsalva maximal diameter was measured perpendicular to the axis of the proximal aorta. The STJ was measured at the point where the sinus of Valsalva continues to the tubular ascending aorta. The tubular aorta was visualized as distally as possible. The maximal diameter perpendicular to the axis of the aorta was taken (figure 1). ${ }^{6}$

\section{Statistical analyses}

Statistical analyses were performed using SPSS version 22 (SPSS Inc. Chicago, IL). Normally distributed continuous variables were reported as mean \pm standard deviation (SD) and non-normal distributed continuous variables as median and interquartile range (IQR). Categorical data are reported as number $(n)$ and percentage (\%). Changes in aortic dimensions over time were modeled using mixed linear model analyses with a random slope and random intercept. Independent variables investigated were age, sex, body surface area (BSA), valve morphology, valvular dysfunction, hypertension, aortic dimension and time. The final models were also analyzed in the presence of potential confounders (sex, BSA and age). Dependent variables were LVOT, sinus of Valsalva, STJ and TA. Potential interactions between time and the other independent variables were also tested. The final models were realized by stepwise elimination using a threshold $p$-value of $<0.10$.

\section{RESULTS}

Population characteristics

In this single center study, 392 patients with a bicuspid aortic valve with serial echocardiographic images available were enrolled. Median follow-up (IQR) was 5 (4) years in which patients underwent $3.6 \pm 1.6$ echocardiographies. $69 \%$ of patients were male and mean age was $48( \pm 17)$ years with a mean BSA of $1.9( \pm 0.2) \mathrm{m}^{2}$ during the first echocardiography (Table 1). According to the raphe-related classification, ${ }^{22} 78 \%(n=305)$ of patients were classified as type $1,19 \%(n=73)$ as type 0 BAV and $2 \%$ $(n=7)$ as type $2 \mathrm{BAV}$. When considering the leaflet fusion type within the raphe-related classification type 1, BAV with a raphe between the RCC and LCC (RCC/LCC subtype) was the most common subtype of BAV in $56 \%$ of the study population ( $n=221$ ), followed by the RCC/NCC subtype in $14 \%(n=54)$. The NCC/LCC subtype of BAV was the least common in this group with $8 \%(n=30)$ of patients. (figure 2$)$. In $2 \%(n=7)$ of the patients, presence of BAV was confirmed, although exact determination of the morphology was uncertain.

\begin{tabular}{|c|c|c|}
\hline & & $\begin{array}{l}\text { Total population } \\
(\mathrm{n}=392)\end{array}$ \\
\hline \multirow[t]{7}{*}{ BASELINE } & Age (years), mean ( $\pm S D$ ) & $48( \pm 17)$ \\
\hline & Male, n (\%) & $271(69)$ \\
\hline & BSA $\left(m^{2}\right)$, mean $( \pm S D)$ & $1.9( \pm 0.2)$ \\
\hline & Hypertension, n (\%) & $155(40)$ \\
\hline & $\begin{array}{l}\text { AR, } n(\%) \\
\quad \text { Mild, } n(\%) \\
\text { Moderate, } n(\%) \\
\text { Severe, } n(\%)\end{array}$ & $\begin{array}{l}269(68.6) \\
218(55.6) \\
49(12.5) \\
2(0.5)\end{array}$ \\
\hline & $\begin{array}{l}\text { AS, } n(\%) \\
\text { Mild, } n(\%) \\
\text { Moderate, } n(\%) \\
\text { Severe, } n(\%)\end{array}$ & $\begin{array}{l}206(52.6) \\
125(31.9) \\
71(18.1) \\
10(2.6)\end{array}$ \\
\hline & $\begin{array}{l}\text { Other congenital heart disease, } \mathrm{n}(\%) \\
\text { Coarctation of the aorta } \\
\text { Atrial septal defect (ASD) } \\
\text { Ventricular septal defect (VSD) } \\
\text { Patent foramen ovale } \\
\text { Patent ductus arteriosus } \\
\text { Transposition of the great arteries } \\
\text { Combined congenital disease* }\end{array}$ & $\begin{array}{l}34(8.7) \\
22(5.7) \\
2(0.5) \\
3(0.8) \\
1(0.3) \\
1(0.3) \\
1(0.3) \\
4(1.1)\end{array}$ \\
\hline \multirow[t]{3}{*}{ FOLLOW-UPt } & Endocarditis, n (\%) & $5(1)$ \\
\hline & Aortic dissection, $\mathrm{n}(\%)$ & $0(0)$ \\
\hline & $\begin{array}{l}\text { Surgical intervention, } n(\%) \\
\text { Combined aortic (valve) surgery, } n(\%) \\
\text { Aortic valve replacement, } n(\%) \\
\text { Aortic valve reconstruction, } n(\%) \\
\text { Supracoronary aortic replacement, } n(\%)\end{array}$ & $\begin{array}{l}58(14.8) \\
16(4.1) \\
39(9.9) \\
2(0.5) \\
1(0.3)\end{array}$ \\
\hline
\end{tabular}

Table 1. Legend on next page 
Table 1. Abbreviations: $A S$, aortic valve stenosis; $A R$, aortic valve regurgitation: $B S A$,

body surface area

*Combined congenital heart disease: coarctation combined with ASD, VSD or patent ductus arteriosus tAll events and surgical interventions were performed after the last echocardiography included in the current study

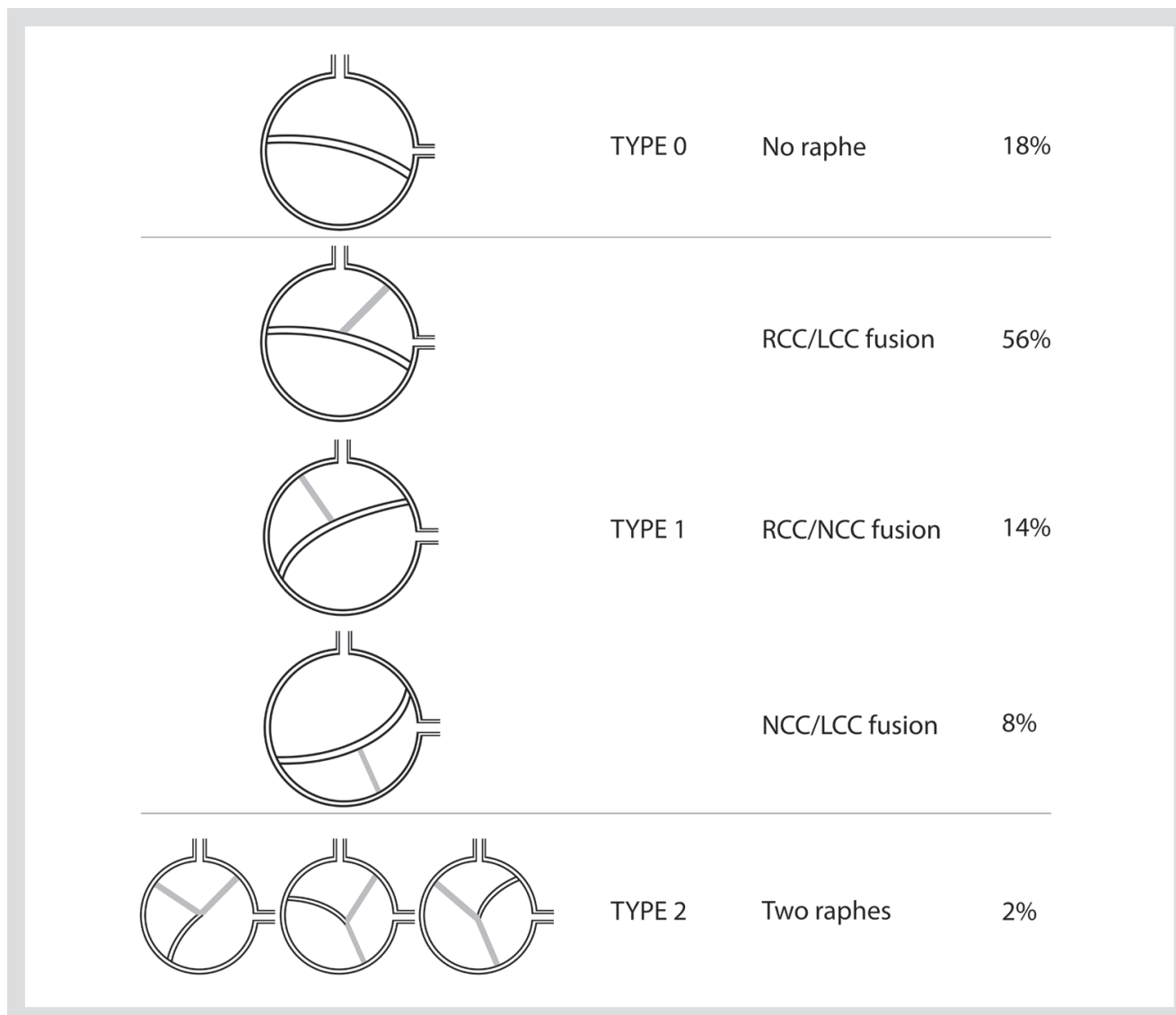

Figure 2. Distribution of the types of BAV by raphe-related and fusion type

Abbreviations: LCC: Left coronary cusp; NCC: non-coronary cusp; RCC: right coronary cusp

\section{Associations with aortic dimensions}

Aortic dimensions per BAV subtype during the first echocardiography are presented in figure 3 . Classification using leaflet fusion type showed an association with all aortic segments. The dimension of the sinus of Valsalva was significantly smaller in patients with the BAV subtype NCC/ LCC when compared to the other fusion types. Furthermore, the RCC/LCC fusion subtype was significantly larger when compared to the RCC/NCC fusion subtype. As for the sinotubular junction dimensions, the NCC/LCC subtype was associated with the smallest dimensions in comparison to

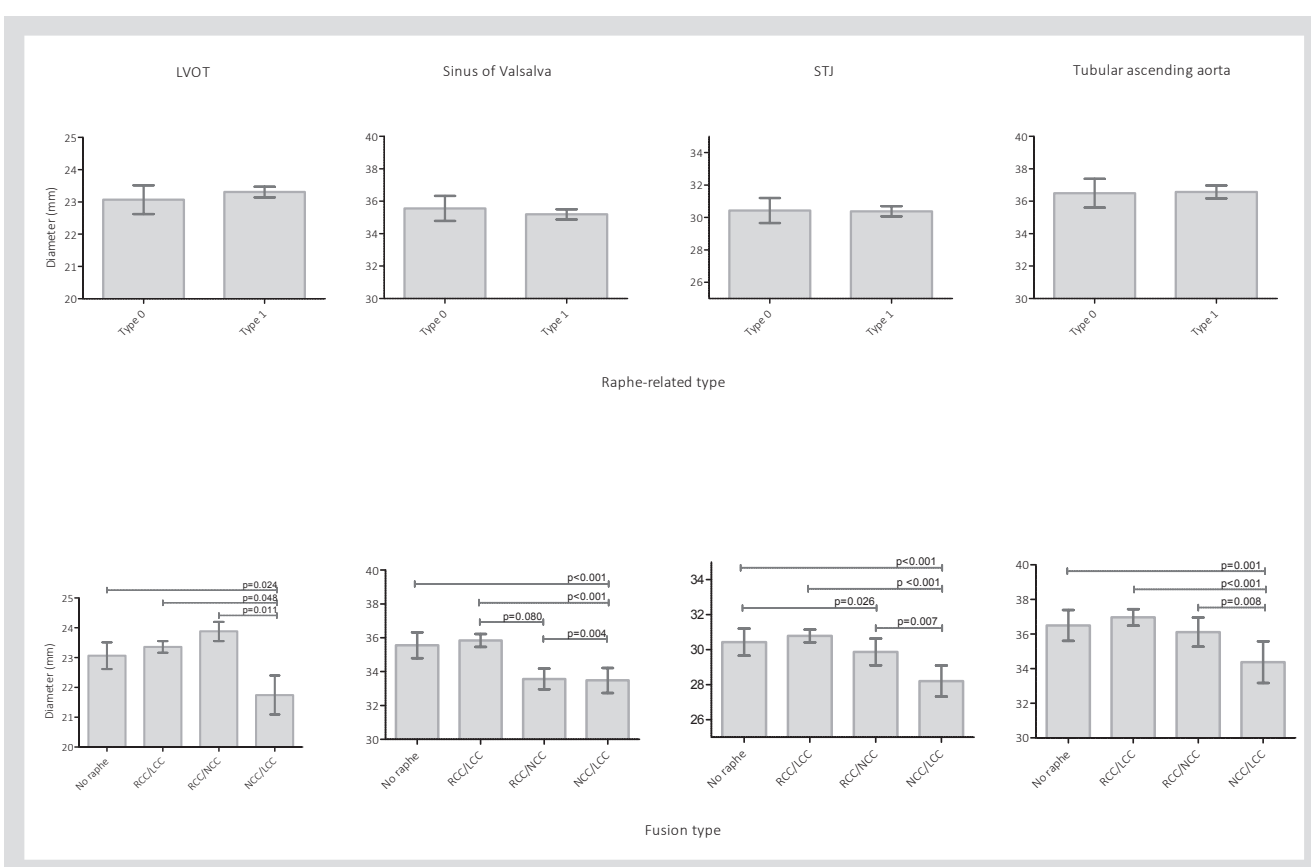

Figure 3. Aortic segment dimensions. Segments from left to right: LVOT, sinus of Valsalva, sinotubula junction (STJ) and tubular ascending aorta. Upper panel: raphe-related classification of BAV. Lower panel: fusion type classification of BAV

the other subtypes and the subtype without a raphe had larger dimensions in comparison to the RCC/NCC subtype. Finally, the NCC/LCC subtype was associated with smaller TA and LVOT dimensions compared to the other subtypes. On the contrary, when patients were classified according to number of raphes, an association between the aortic dimensions with BAV morphology in the model was not found.

Presence of an association between aortic dimensions and other biologically plausible parameters was investigated. The significantly associated parameters for all aortic dimensions are listed in table 2. Dimensions of all segments were significantly larger in males. A high BSA was associated with significantly larger dimensions of the LVOT, sinotubular junction and tubular ascending aorta. Increasing age was associated with larger dimensions of all segments except for dimensions of the LVOT. We further investigated the association between valvular dysfunction and aortic dimensions and found that the presence of aortic regurgitation (AR) or aortic valve stenosis (AS) was associated with larger LVOT dimensions or TA dimensions respectively. Dimensions of the other aortic segments were not associated with AR or AS. 

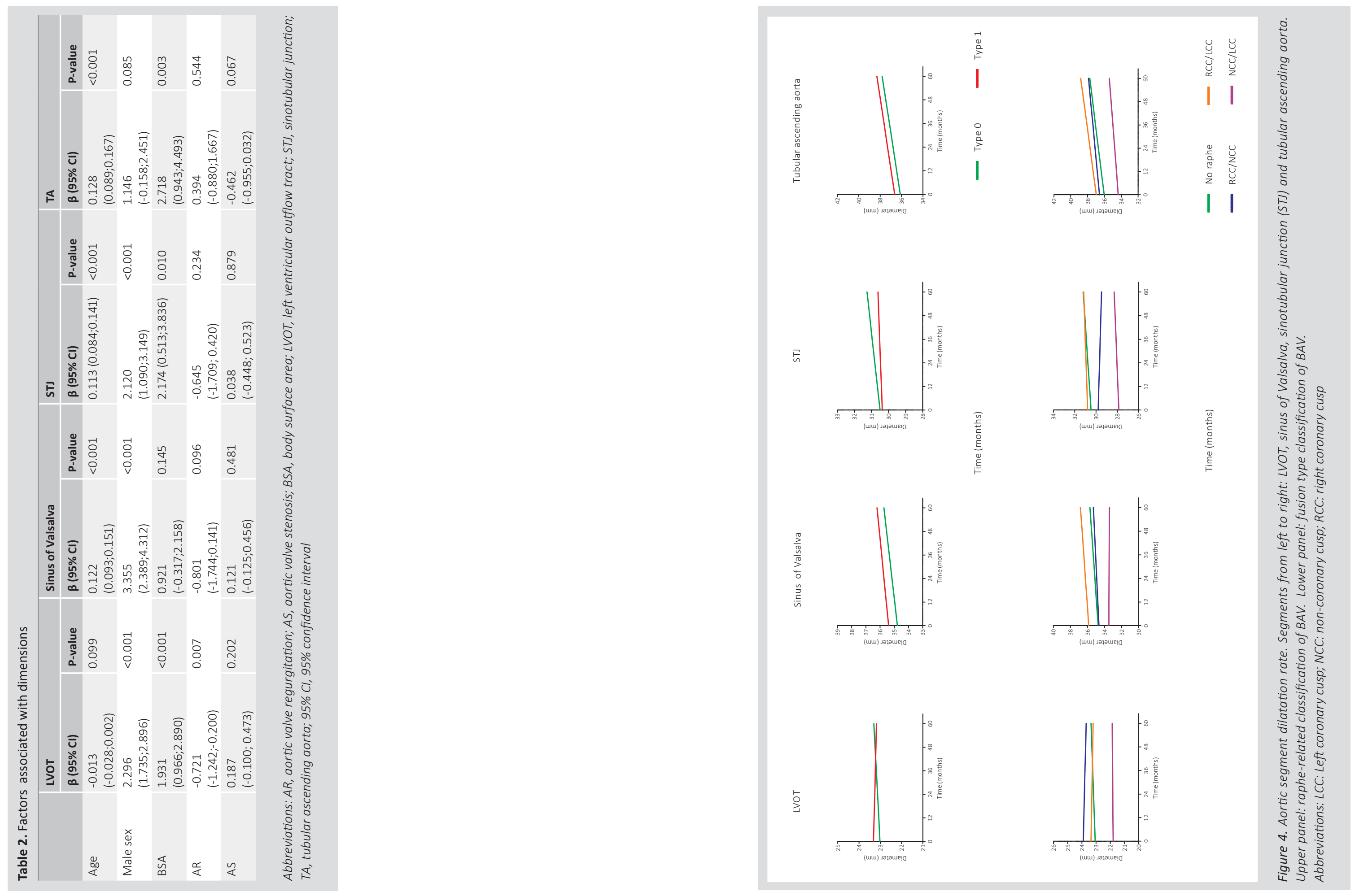

Chapter 10 


\section{Aortic dilatation rate variation}

Serial assessments of aortic dimensions were used to determine the dilatation rates of the aortic segments, employing mixed linear models. Mean aortic dilatation rates differed across aortic segments. The mean ( \pm SD) increase of the sinotubular junction was $0.06( \pm 0.05) \mathrm{mm} / \mathrm{year}$, the mean increase of the sinus of Valsalva was $0.18( \pm 0.02) \mathrm{mm} /$ year and the tubular ascending aorta showed the fastest dilatation rate: $0.32( \pm 0.03) \mathrm{mm} /$ year. The LVOT did not show significant annual progression of dilatation

We next investigated which parameters were associated with increased aortic dilatation rates. BAV morphology type was not associated with aortic dilatation in any of the segments mentioned above. This finding is illustrated by similar slopes in figure 4, representing growth per morphology type. Baseline dimensions of the tubular ascending aorta were associated with dilatation rate of that specific aortic segment but this association could not be found in the other segments. As for the other parameters a significant association with dilatation rates of any of the aortic segments could not be demonstrated. Addition of potential confounders (age, sex and BSA) to the model did not affect the results (data not shown).

\section{Monocuspid valves, a special subgroup}

Two percent $(n=7)$ of the aortic valves in this study population was monocuspid. Overall, the presence of a monocuspid aortic valve seemed to result in larger dimensions of the aorta in al segments, but did not show morphology-dependent dilatation rates as well. The small number of patients necessitates cautious interpretation of these data, as they may not be representative for the population with a monocuspid aortic valve as a whole.

\section{DISCUSSION}

The aim of the present study was to elucidate on determinants of dimensions and dilatation rates of ascending aortic segments in a population consisting of 392 patients with a bicuspid aortic valve. The main findings of this study are as follows: First, segments of the proximal ascending aorta showed different dilatation rates. The tubular ascending aorta showed the highest dilatation rate of $0.32 \mathrm{~mm} /$ year, followed by the sinus of Valsalva with $0.18 \mathrm{~mm} / \mathrm{year}$ and the sinotubular junction with a dilatation rate of $0.06 \mathrm{~mm} / \mathrm{year}$. Second, type of BAV morphology showed no association with aortic dilatation rates, regardless of the classification system (raphe-related type or fusion type). The association between valve morphology and aortic dilatation rates has been suggested and contradicted in previous studies and shows a wide dispersion of dilatation rates.2,21, Our study results are in accordance with recent studies, regarding dilatation rates in BAV aortopathy. However, we believe that the results of this study have added value in the confirmation of results of these studies, 19,20 due to group size and the strong method of analyses. Mixed linear models provide an elegant way to overcome different follow-up time intervals and provide a solid basis to underpin the dilatation rates found in this study by not only taking the first and last measurement of the aortic dimensions into account, but all intermediate measurements as well, eading to a more accurate estimate of the aortic dimensions and dilatation rates over time. Third, morphology types were associated with differences in dimensions of the proximal aorta. The association between BAV morphology and aortic size has been subject of extensive debate, especially since initial studies did not find an association whereas in later studies morphology related significantly to both sinus of Valsalva and TA size. $18,25,26$ The present findings are in accordance with the latter and support the notion that BAV morphology related hemodynamics may cause aortic dilatation directly, but does not exclude a role for underlying ontogenetic defects or a role of an interplay between both. The involvement of genetic defects and defects in the neural crest cells leading to the development of BAV (calcification) and concomitant abnormalities of the aorta by disruption of the extracellular matrix ${ }^{27-30}$ have been studied in conjunction with hemodynamic patterns in BAV disease..$^{31,32}$ A cross-sectional study by Hope et al.16 showed an eccentric flow resulting in an abnormal helical flow pattern in a subset of patients with and without aortic dilatation and a bicuspid aortic valve with "normal" function. They suggested a role for hemodynamic stress in identifying patients at risk for developing aortic aneurysms, taking into account the alternative hypothesis that presence of intrinsic aortic wall abnormalities predisposes to a greater aortic dilatation in the presence of abnormal hemodynamic stress. ${ }^{33}$ Fourth, our modeled analyses show that dimensions of the aortic segments are influenced by sex, age, BSA and valvular function, whereas dilatation rates are not. The inability to show this association does not aid in optimizing risk stratification in BAV aortopathy. As a result of the unpredictability of dilatation rates, an especially challenging aspect of BAV is the timing of surgical intervention. ${ }^{3}$ Verma et $a^{10}$ found significantly different clinical decisions by cardiac surgeons towards optimal timing of surgical intervention. This group recommended a renewed strategy for follow-up and timing of aortic surgical interventions, accepting more dilatation of the aorta (in absence of risk factors). Biological/genetic background might play a more important role in dilatation rates and should probably be taken into account when deciding on timing of follow-up and aortic surgery. A positive family history has been described as a denominator of risk as well. ${ }^{19}$ For now, it still is a matter of debate whether the timing of surgery in BAV should be based on absolute dimensions. A novel model for timing of follow-up and surgical intervention fed by data from observationa studies incorporating the above aspects including genomics, may improve surgical care for BAV patients but obviously needs validation in prospective trials.

\section{Study limitations}

Although the study population size was relatively large and the aortic measurements were repeated prospectively by two independent observers, some possible limitations merit attention.

Despite the size, this study is prone to some bias due to its retrospective observational design On the other hand, our hospital is a combined regional and tertiary center serving its own population. With that, we assume we evaluated a representative population when compared to populations in exclusively tertiary centers.

Another limitation is inherent to the serial echocardiographic measurements. Measurements are dependent on image quality and availability of echocardiographic images. We tried to minimalize bias based on image quality by requiring valid measurements from independent observers.

Aortic dilatation is a lifelong process, necessitating long term follow-up in clinical studies. Thus, aortic dilatation is frequently studied in retrospective cohorts. Although the retrospective study design inherently involves some limitations, the current manuscript represents the natura clinical course of BAV patients within our institution. These results have to be interpreted with caution in a population with a tricuspid aortic valve, since this group was not included in the current study and might show another natural clinical course of aortic dilatation when compared to patients with BAV.

Finally, despite the relatively large population size, the group of patients with a monocuspid aortic valve was relatively small and reliability of the analyses considering this group was considered low and was not included in this study. 


\section{CONCLUSION \& CLINICAL IMPLICATIONS}

The prediction of aortic complications resulting from (asymptomatic) aortic dilatation rates and timing of surgical intervention is a major challenge in patients with $B A V^{5,8}$ necessitating long-term and costly follow-up. Dilatation rates in BAV aortopathy vary widely among patients with a maximum dilatation rate of the tubular ascending aorta, followed by the sinus of Valsalva and the sinotubular junction. Dilatation rates are not associated with BAV morphology, and thus BAV morphology should not be used for additional risk stratification. A small group of patients might benefit from a stricter follow-up to determine progression of dilatation at an early stage. Moreover, the risk of developing aortic complications should be determined individually durin follow-up echocardiography. Finally, there is an unmet clinical need for improvement of risk stratification in BAV patients. Genomics is expected to gain importance in this field.
REFERENCES on. 2015;131(4):e29-322.

A, Bancone C, Buonocore M, et al. Pattern of ascending aortic dimensions predicts the growth rate of diovascular imaging. 2013;6(12):1301-1310.

3. Michelena HI Desjardins VA, Avierinos IF, et a Naturathis tory of asymptomatic patients with normally functioning or munity. Circulation. 2008;117(21):2776-2784.

4. Tzemos $\mathrm{N}$, Therrien J, Yip J, et al. Outcomes in adults wi bicuspid aortic valves. Jama. 2008;300(11):1317-1325,

5. Nishimura RA, Otto CM, Bonow RO, et al. 2014 AHA/ACC Heart Disease: executive summary: a report of the America College of Cardiology/American Heart Association Task Force on Practice Guidelines. Circulation. 2014;129(23):2440-2492. 6 Hiratzka LF, Bakris GL, Beckman JA, et al. 2010 ACCF/AHA/ AATS/ACR/ASA/SCA/SCA/SIR/STS/SVM guidelines for the Diagnose: a report of the American College of Cardiorto Foundation/American Heart Association Task Force on Practice Guidelines, Americon Association for Thoracic Surgery, American College of Radiology, American Stroke Association, Society of Cardiovascular Anesthesiologists, society for Cardiovascular Angiography and Interventions, (arculation. 2 2010;121(13):e266-369

7. Joint Task Force on the Management of Valvular Heart Disease of the European Society of C, European Association for Cardio-Thoracic S, Vahanian A, et al. Guidelines on the European heart journal. 2012;33(19):2451-2496.

8. Erbel R. Aboyans V Boileau C et at 2014 ESC Gudelines on the diagnosis and treatment of aortic diseases: Document covering acute and chronic aortic diseases of the thoracic and abdominal aorta of the adult. The Task Force for the Diagnosis and Treatment of Aortic Diseases of the European Society of Cardiology (ASC) Fusopeon

Acc//hha/Aats/Acr/Asa/Sca/Scai/Sir/Sts/Svm Guidelines Disease Representative M, Hiratzka LF, et al. Surgery for Aortic Dilatation in Patients With Bicuspid Aortic Valves: $A$ Statement of Clarification From the American College of Cardlology/American Heart Association Task Force on Cln-

Verma S, Yanagawa B, Kalra S, et al Knowledge, atitudes, and practice patterns in surgical management of bicuspid aortopathy: A survey of 100 cardiac surgeons.J Thorac Car- 26. diov Sur. 2013,146(5).1033-1040.

11. Nistri S, Sorbo MD, Marin M, Palisi M, Scognamiglio R, Thiene G. Aortic root dilatation in young men with normally

12. Yasuda $\mathrm{H}$ Nakatani S, Stugaard $\mathrm{M}$, et al Falure to prevent progressive dilation of ascending aorta by aortic valve replacement in patients with bicuspid aortic valve: com- 28. Suppl 1:11291-294

13. Fedak PW, Vesma S, David TE, Leask RL, Weisel RD, Butany lar matrix protein transport associated with increased drome and bicuspid aortic valve thoracic aortic aneurysm. Circulation. 2003;108 Suppl 1:11329-334,

Bum Is NS, Hope MD. Bicuspid valve-related aortic disease

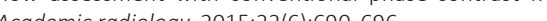

Hope MD, Hope TA, Meadows AK, et al. Bicuspid aortic valve: four-dimensional MR evaluation of ascending ao
systolic flow patterns. Radiology. 2010:255(1):53-61.

Kang JW, song HG, Yang DH, et al. Association between bicuspid aortic valve phenotype and patterns of valvular uation usin MDCT and echocardiography. IACC. Cardiovascular imaging. 2013;6(2):150-161.

Schaefer BM, Lewin MB, Stout KK, et al The bicuspid aortic valve: an integrated phenotypic classification of leaflet morphology and aortic root shape. Heart. 2008;94(12):1634-1638. Avadhani SA, Martin-Doyle W, Shaikh AY, Pape LA. Predicease: a five-year prospective study. The American journal of medicine. 2015;128(6):647-652.

Detaint D, Michelena HI, Nkomo VT, Vahanian A, Jondea $G$, Sarano ME. Aortic dilatation patterns and rates in adults with bicuspid aortic valves. a comparative study with Marfan syndrome and degenertive

W Filion K, et al. Retrospective stud 作 valves. Nature clinical practice. Cardiovascular medicine. 2008; $5(12): 821-828$.

biers $\mathrm{HH}$, Schmidtke C. A classification system for the 2. 2007;133(5):1226-1233

3. Baumgartner H, Hung J, Bermejo J, et al. Echocardiographic assessment of valve stenosis: EAE/ASE recommendation for clinical practice. Journal of the American Society of Echocardiography: Official publication of the American

. Holmes KW, Lehmann CU, Dalal, etal Progessive diltion of the ascending aorta in children with isolated bicuspid aortic valve. The American journal of cardiology. 2007;99(7):978-983.

. Jassal DS, Bhagirath KM, Tam JW, et al. Association of Bicuspid aortic valve morphology and aortic root dimensions: measuring effects of rosuvastatin (ASTRONOMER) study Echocardiography. 2010;27(2):174-179.

. Cecconi M, Nistri S, Quarti A, et al. Aortic dilatation in patients with bicuspid aortic valve. Journal of cardiovascular medicine. 2006; 7(1):11-20.

. implications for aortic dilatation. The Journal of thoracic and cardiovascular surgery. 2003;126(3):797-806.

Fedak PW, Verma S. The molecular fingerprint of bicuspid artopathy. The Journal of thoracic and cardiovascular surgery. 2013;145(5):1334.

.

30. Ikonomidis JS, Jones JA, Barbour JR, et al. Expression of 
matrix metalloproteinases and endogenous inhibitors within ascending aortic aneenrysms of patients with bicus. Broberg CS. Therrien J. Understanding and tree.

athy in bicuspid aortic valve. Trends in cardiovascular medcine. 2015;25(5):445-451.

2. Tadros TM, Klein MD, Shapira OM. Ascending aortic dilatation associated with bicuspid aortic valve: pathophysioogy, molecular wiolosy, and anical

Ferencik M, Pape LA Change

and aortic valve function with time in ascending aorta genitally bicuspid aortic valves. The American journal of cardiology. 2003;92(1):43-46,

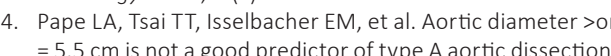
$=5.5 \mathrm{~cm}$ is not a good predictor of type A aoortic dissection Dissection (IRAD) Circulation. 2007:116(10):1120-1127. 
GENERAL DISCUSSION 
This thesis highlights the complexity of aortic valve disease and its progression. Steps were undertaken to find and unravel a piece of the aortic stenosis puzzle; a puzzle that, when completed, should lead to improved insights in AS, ultimately leading to an approach with integrated circulating and imaging biomarkers to enable monitoring and development of therapies to halt progression. In other words: to stop the "snowball effect" of aortic valve stenosis.

\section{THE INTERPLAY OF MECHANISMS IN AORTIC VALVE STENOSIS AND POTENTIAL}

\section{TARGETS FOR MEDICAL THERAPY}

In Chapter 2, we summarize the substantial transformation in knowledge regarding the pathophysiology of aortic valve stenosis (AS) over the past decades. ${ }^{1}$ Traditionally, AS was considered a passive process of wear and tear resulting in calcification within the valve leaflets, but today, AS is appreciated to be regulated by the interaction of a highly complex series of pathophysiological processes. In particular, two phases are recognized in AS: the initiation and progression phase, each dominated by different mechanisms. The classical view on the initiation phase is endothelia damage triggering lipid infiltration and oxidation, provoking an inflammatory response involving macrophages, T-lymphocytes, and mast cells. Inflammation triggers phenotypic switching of valvular interstitial cells (VIC) resulting in increased extracellular vesicle release, providing a nidus for (micro)calcification. Microcalcification, in turn, provokes an inflammatory response, resulting in increased apoptosis and/or delayed phagocytosis, thereby expanding calcium deposition..$^{2-1}$ Upon AS propagation, pro-fibrotic and pro-calcific processes predominate. Pro-fibrotic changes leading to collagen deposition and facilitating progressive calcification are mediated by reduced nitric oxide expression and up-regulation of renin-angiotensin system. ${ }^{11-14}$ Calcification is considered to be the dominant process driving disease progression. VIC phenotype switching to an osteoblas phenotype is thought to play a fundamental role in the propagation phase and is driven by multiple regulatory pathways (for instance Notch1, RANK/RANKL/OPG, Wnt/b-catenin). Bone morphogenetic is a crucial protein in this phenomenon of VIC phenotype switching. ${ }^{15-21}$ Physiologically, Matrix Gla Protein (MGP), a vitamin K dependent protein in need of carboxylation to be activated (CMGP), inhibits BMP-2.22 The vital role of MGP in vascular calcification was demonstrated in 1997 already, 23,24 and more recently it was shown that decreased expression or activity of MGP contributed to the progression of vascular and valvular calcification. Besides forming a complex with BMP-2 and by that inhibiting the BMP-2 pro-mineralizing activity, MGP exerts its function via a second mechanism by directly binding to hydroxyapatite and inhibiting growth of crystals. $22,25-29$ Hence, the work in this thesis provides a novel basis that not only disease progression but also initiation is driven by calcification (Chapter 2 and 6).

The inhibitory function of MGP in AS (micro)calcification and progression seems evident by its interactions with both BMP-2 and hydroxyapatite, making it a potential target for medical intervention. Therefore, we posed two separate issues requiring further investigation, being (1) the presence of a counter-mechanism enhancing calcification, and (2) the effect of supplementing vitamin $\mathrm{K}$ in patients with AS to decrease progression.

The central question in the counter mechanism mentioned in the first issue is whether calcification is enhanced upon vitamin $\mathrm{K}$ depletion, leaving us with biologically inactive (uncarboxylated) MGP. As mentioned above, MGP is a vitamin $\mathrm{K}$ dependent protein. Vitamin $\mathrm{K}$ serves as an essential cofactor enabling Y-carboxylation, thereby activating MGP. Vitamin Kantagonists (VKA) block the -carboxylation, hence inhibiting full bioactivity of MGP, resulting in potentially decreased tissue calcification protection. . $430-32$ We studied this hypothesis in a propensity-score matched population including patients with lone atrial fibrillation (AF) with low cardiovascular risk profiles treated with vitamin antagonists (VKA), non-vitamin K antagonist oral anticoagulants (NOAC) and patients not treated with ral anticoagulation (Chapter 3). In patients with similar risk profiles, we found that VKA treatment was associated with a higher prevalence of calcification of the aorta and aortic valve as compared to patients not treated with VKA. Price et al investigated this phenomenon in 1998 already and found that VKA caused calcification of vascular and valvular tissue in rats treated ${ }^{24}$ In addition, we found a trend in the duration of VKA treatment towards an increase in calcification, hinting towards a direct calcification-inducing effect of VKA. At last, differences in calcification between NOAC treated patients and patients not treated with oral anticoagulation were not observed. Although limitations of this study have to be taken into account (short duration of NOAC treatment versus VKA treatment duration, relatively small propensity score-matched groups and the cross-sectional character of the study), this study added evidence to existing knowledge regarding the "calcification-induction effect" of VKA and suggested an absence of this effect of NOACs. Concerning the association between calcification and VKA treatment, results are in accordance with former studies (including patients with different risk profiles), while results regarding the time-dependent relation were controversial in earlier studies, necessitating further research. ${ }^{33,34}$ Moreover, prospective longitudinal studies and randomized controlled trials will allow determining the true absence of an association between NOAC treatment and calcification. ${ }^{35} \mathrm{~A}$ recent study investigating this association in mice treated with Rivaroxaban or Warfarin indeed reported that mice treated with Rivaroxaban had similar calcification burden as control mice and showed calcification to a lesser degree as compared to Warfarin treated mice. ${ }^{36}$

Several studies provided a clue regarding the second issue by providing evidence that (supplementation of) vitamin $\mathrm{K}$ was associated with inhibition of calcification. ${ }^{37-40}$ Therefore, as a secondary hypothesis, we hypothesized that supplementation of vitamin $\mathrm{K}$ bears potential to inhibit the progression of calcification in AS through activation of MGP. Chapter 4 and 5 investigated this hypothesis. In Chapter 4, the results of the effect of supplementation with Vitamin K1 (Phytomenadione, VK1, $2 \mathrm{mg}$ orally daily) were presented in a first-in-man proof-of-concept trial using calcification volume scoring on cardiac computed tomography (CT) after one year. Significant attenuation of progression of aortic valve calcification was observed in the VK1 arm as compared to placebo, suggesting the potential role of vitamin $\mathrm{K}$ supplementation in aortic valve stenosis. ${ }^{41}$ Chapter 5 comprises a rationale and trial design of an on-going trial, investigating the effect of vitamin $\mathrm{K} 2$ (Menaquinone-7; MK7) in patients with a bicuspid aortic valve and aortic valve stenosis. ${ }^{42}$ In this chapter, other studies investigating potential pharmacological treatment were outlined, as well as trials currently running. Unfortunately, until now, negative and controversial results have dominated in the field of other treatment options. In the BASIK2 trial ("Bicuspid Aortic Valve Stenosis and the Effect of Vitamin K2 on Calcification Using ${ }^{18} \mathrm{~F}$-Sodium Fluoride Positron Emission Tomography/ Magnetic Resonance") the effect of MK7 on aortic valve calcification will be investigated using ${ }^{18} \mathrm{~F}$-sodium fluoride $\left({ }^{18} \mathrm{~F}-\mathrm{NaF}\right)$ positron emission tomography (PET)/magnetic resonance (MR). During trial set-up, multiple aspects needed to be considered, such as the choice to investigate aortic valve stenosis in bicuspid valves instead of tricuspid valves, supplementation with MK7 instead of VK1 and the imaging methods to assess the potential effect. In short, bicuspid aortic valves are predisposed to accelerated calcification and progression of stenosis, ${ }^{43-45}$ and MK7 has a significantly higher bioavailability and bioactivity in vivo compared to vitamin $\mathrm{K} 1 .{ }^{46} \mathrm{At}$ last, the rationale to evaluate the effect of MK7 by ${ }^{18} \mathrm{~F}-\mathrm{NaF}$ tracer uptake instead of conventional calcium scoring on $\mathrm{CT}$ has been provided in both Chapter 5 and Chapter 2. ${ }^{18} \mathrm{~F}-\mathrm{NaF}$ enables visualization of initiating microcalcification, which in turn may help to identify location and extent of eventual macrocalcification and thereby predict future aortic valve stenosis progression in an early phase. ${ }^{1,47-50}$ Although both studies provide us with new and important insights, both are proof-of-concept trials, and thus in need of further validation in future trials to confirm the effect on hemodynamic parameters and clinical outcomes. 


\section{FROM UNRAVELING MECHANISMS AND BIOMARKERS TO APPLICATION OF CIRCULATING AND IMAGING BIOMARKERS: SLIDING FROM BENCH}

\section{TO BEDSIDE}

The progressive character of AS and the absence of a medication-based treatment triggered cardiovascular research to identify the exact mechanism underlying aortic valve calcification and interactions between pathways. $1,18,19,50-52$ In general, the translation of new understandings of disease mechanisms into clinically useful applications in diagnosis, therapy and prevention requires information sharing between laboratory and clinic, also known as a simplified definition of "translational research" or taking research "from bench-to-bedside". ${ }^{33,54}$ As part of translational research, research focusing on the differences between normal (physiological) and abnormal (pathophysiological) mechanisms will pave the road for the development of medical treatment targeted to specific mechanisms involved in AS. In this respect sex differences are of importance. Not only insights in AS disease mechanisms, but also the development of a noninvasive method capable of predicting the natural course of AS in a single patient are part of translational research, as they will provide a major step towards tailored patient care and management approach. In Chapters 6 to 9, we describe studies gently sliding from bench-to-bedside with potential clinical future implications.

A step forward in understanding the role of MGP in the mechanisms involved in AS was investigated in Chapter 6. As part of ongoing analyses, a series of immunohistochemical stainings were performed in a population of 50 patients undergoing aortic valve replacement. In these valves, heavily calcified regions and regions with less calcification were identified according to Von Kossa staining positivity. We showed that UCMGP was present in the borders and regions surrounding calcifications, in contrary to other calcification stainings (Von Kossa and Osteocalcin). Although the cross-sectional design of this study has to be taken into account, these findings suggest MGP be present in regions of active calcification and thereby, its involvement in calcification in AS. Other studies supporting these findings found a significant decrease in active MGP in isolated VICS when compared to normal VICS, reinforcing the ability of MGP to negatively regulate calcification in AS. ${ }^{29,55}$

Whereas Chapter 6 is devoted to the basic side of science to support insight in disease mechanisms, Chapter 7 moves along the translational chain by combining insights in pathophysiology and imaging tools to reveal sex-related differences in predominant processes. Even though calcification is considered the predominant mechanism in AS, the contribution of fibrosis to the progression of AS is a matter of debate. That is, several studies showed aortic valves of female patients with severe AS show less aortic valve calcification on $\mathrm{CT}$ as compared to male patients with similar hemodynamic severity. ${ }^{66-59}$

This disproportion has been a critical issue in clinical practice and as a consequence, different thresholds to confirm AS severity in males and females were implemented. ${ }^{13,57,60}$ However, implementation of different thresholds for calcification burden did not explain the similar hemodynamic severity observed in both sexes. This led to the hypothesis that fibrosis burden might explain the basis of this sex-related discrepancy. Recently, a shift in calcification:fibrosis ratio was demonstrated in patients with varying degrees of AS by CT and histology. ${ }^{61}$ However, histologic assessment is hampered by the need for valve tissue, mostly derived from surgery in severe AS. In our study (Chapter 7), we confirmed the calcification:fibrosis ratio shift, and suggest a dominant fibrotic phenotype in females with AS (calcification:fibrosis ratio<1), whereas males tend to show dominance in calcific phenotype. Additionally, our results are based on the application of a combined noninvasive clinical imaging technique solely, thus not necessitating histology. More specifically, fibrosis, calcification and calcification activity were quantified using ${ }^{18} \mathrm{~F}$-sodium fluoride $\left({ }^{18} \mathrm{~F}-\mathrm{NaF}\right)$ positron emission tomography (PET) and contrast-enhanced CT. ${ }^{18} \mathrm{~F}-\mathrm{NaF}$ is known as a biomarker that binds to regions of developing calcification, reflecting calcification activity and progression of AS. ${ }^{47,49}$ Although further confirmation is needed in longitudinal studies, our results highlight the potential application of ${ }^{18} \mathrm{~F}-\mathrm{NaF}$ PET/CT to measure disease activity and fibrosis in patients with AS. Moreover, given the fact that PET allows measurement of activity of specific biological processes within specific structures and that ${ }^{18} \mathrm{~F}-\mathrm{NaF}$ is a suitable tracer reflecting calcification activity, application of this technique enables us to investigate processes of AS in more detail in early phases of the disease. ${ }^{3,62}$

In line with Chapter 7, Chapter 8 explored sex-related differences in the expression of a panel of circulating biomarkers in a small study including low-risk patients in an early phase of aortic valve calcification and found higher expression of fibrosis markers in females, whereas differential expression of calcification and inflammatory markers were found in males. Again, this data adds information to the knowledge of mechanisms involved in aortic valve calcification, but results have to be interpreted with caution. This study does amplify the complexity of aortic valve calcification and stenosis and provides a rationale to integrate a multi-biomarker approach in longitudina studies in aortic valve stenosis.

As mentioned above, imaging biomarkers have been investigated for their established or potential role in the diagnosis and follow-up in AS. Studies investigating the potential role of circulating biomarkers in follow-up in AS are scarce though, and current guidelines only recommend repeated measurements of markedly elevated natriuretic peptides. Whilst these are incorporated in the most recent guidelines, their actual role in clinical management is not clearly defined. ${ }^{60}$ Emerging studies investigate the potential utility of other biomarkers, such as troponin-T, troponin-I, ST2, growth differentiation factor-15 (GDF-15) and galectin-3 showing variable results. ${ }^{63-69}$ A recent study investigating multiple biomarkers of cardiovascular stress revealed that a combination of GDF-15, SST2, and NT-proBNP provided prognostic implications in patients with $\mathrm{AS}$, and with that, a net improvement in risk stratification for mortality after both conventional aortic valve replacement and TAVI. ${ }^{70}$ Therefore, it seems likely that multiple biomarkers reflecting various disease mechanisms will be useful in AS. Moreover, we hypothesize that measurement of these biomarkers holds potential as a complementary approach to gain insight in AS progression and timing of intervention in future clinical care. When circulating biomarkers are repeatedly measured, changes between measurements should reflect disease progression ideally. However, the interpretation of whether an observed change over time is clinically relevant is challenging, and knowledge about their natural variation is essential. ${ }^{71}$ In Chapter 9, we presented the first study investigating this variation (known as biological variation) in a patient group with stable AS using serial measurements of potentially us eful biomarkers: NT-proBNP, BNP, troponin-T, troponin-I and ST2. Indices of biological variation found in this group approximated those found in both healthy and chronic heart failure populations. ${ }^{72-77}$ Moreover, we explored biomarker patterns in patients with progressive AS and although these findings merit further confirmation in larger studies, they hold potential to distinguish biomarkers suitable for serial measurements in patients to predict progression in AS.

\section{BICUSPID AORTIC VALVES AND OTHER COMPLICATIONS}

Bicuspid aortic valve (BAV) is well known for its heterogeneous presentation and its association with valvular and vascular morbidity. Not only early development and progression of aortic valve calcification and stenosis is common, ${ }^{43-45}$ but dilatation of the thoracic aorta has rather frequently 
been observed in patients with BAV78-81 The natural course of dilatation varies widely, and underlying mechanisms are incompletely understood, challenging indication and timing of elective aortic surgical intervention. ${ }^{82-86}$ Several studies investigated potential determinants of aortic dilatation and reported obvious disparities. ${ }^{87-91}$ Therefore, we studied aortic dilatation rates and associated risk factors, including BAV morphology in a population of 392 patients with longitudinal follow-up in Chapter 10.92 Dilatation rates varied substantially and were not associated with BAV morphology. Other studies attempting to associate dilatation rates by categorizing according to valve morphology showed controversial results. ${ }^{87,939-95}$ Although our study consisted of the largest number of BAV patients with longitudinal follow-up at the time, a recent study included 852 patients in a cross-sectional cohort to assess determinants of valvular dysfunction and patterns of aortic dilatation once more. Again, large heterogeneity in both valvular dysfunction and aortic dilatation was found. They reported that both hemodynamic and demographic variables contributed to valvular dysfunction and aortic dilatation 90 but with significant overlap between valve types. Therefore, it is questionable whether these associations offer enough discrimination to tailor follow-up strategies to valve morphology/type. ${ }^{8}$ Integration of hemodynamic parameters and genetics might better explain the observed heterogeneity in BAV patients. ${ }^{86,87,89,96,97}$ Research integrating both domains holds promise to identify high-risk patients and with that, to offer tailor-made follow-up strategies. ${ }^{86,98}$ In current daily practice, however, patients with aortic dilatation as well as valvular dysfunction are subjected to an identica non-personalized follow-up scheme still devoid of these promising predictors.

\section{FUTURE PERSPECTIVES}

Multiple aspects of aortic valve stenosis were highlighted in this thesis (Figure 1). Our finding covered part of the translational research spectrum and resulted in new challenges and new opportunities for ongoing and future research. In the last decade, inexhaustible groups studying As led to a better understanding of the biomolecular mechanisms involved in AS and novel avenues have started to open for diagnosis and treatment. Still, there is a long road ahead and controversies and questions to be discussed and answered. Integration of both basic and clinical research is fundamental to reach the ultimate goal: generate medical therapies capable of slowing or halting AS progression. Multiple steps and feedback-mechanisms have to be involved to transfer new understandings of AS mechanisms to the integration and development of accurate methods of diagnosis and treatment. Several studies are currently ongoing by our group and others in these fields. In the cohort study mentioned in Chapter 6, local and systemic factors will be studied to explore mechanisms involved in AS. With that, this study aims to provide clues for new amenable targets. In the ongoing randomized controlled trial, BASIK2 (Chapter 5), we investigate the effect of vitamin $\mathrm{K} 2$ on calcification activity in AS using serial ${ }^{18} \mathrm{~F}-\mathrm{NaF}$ PET/MR imaging. With that, this trial does not only investigate the early efficacy of a potential treatment option but also if PET imaging would be best suited to visualize processes involved in AS. Although PET showed to predict AS progression well, it is questionable whether this slightly advanced technique, instead of a rather simple technique alike CT calcium scoring, should be used as a clinical tool reflecting the natura course of AS in future routine clinical care. Ongoing and future trials will address this aspect, as well as the integration of single or combinations of circulating biomarkers to predict progression and timing of intervention. Moreover, other potential agents interfering with the inflammatory, fibrotic and calcification mechanisms such as bisphosphonates and denosumab (SALTIRE II), Niacin and Proprotein convertase subtilisin/kexin type 9 (PCSK9) inhibitors are currently investigated. The results of these trials will shed further light on the relevance of the respective mechanisms and the application of imaging modalities in AS. That is, to stop the snowball effect in aortic valve stenosis.

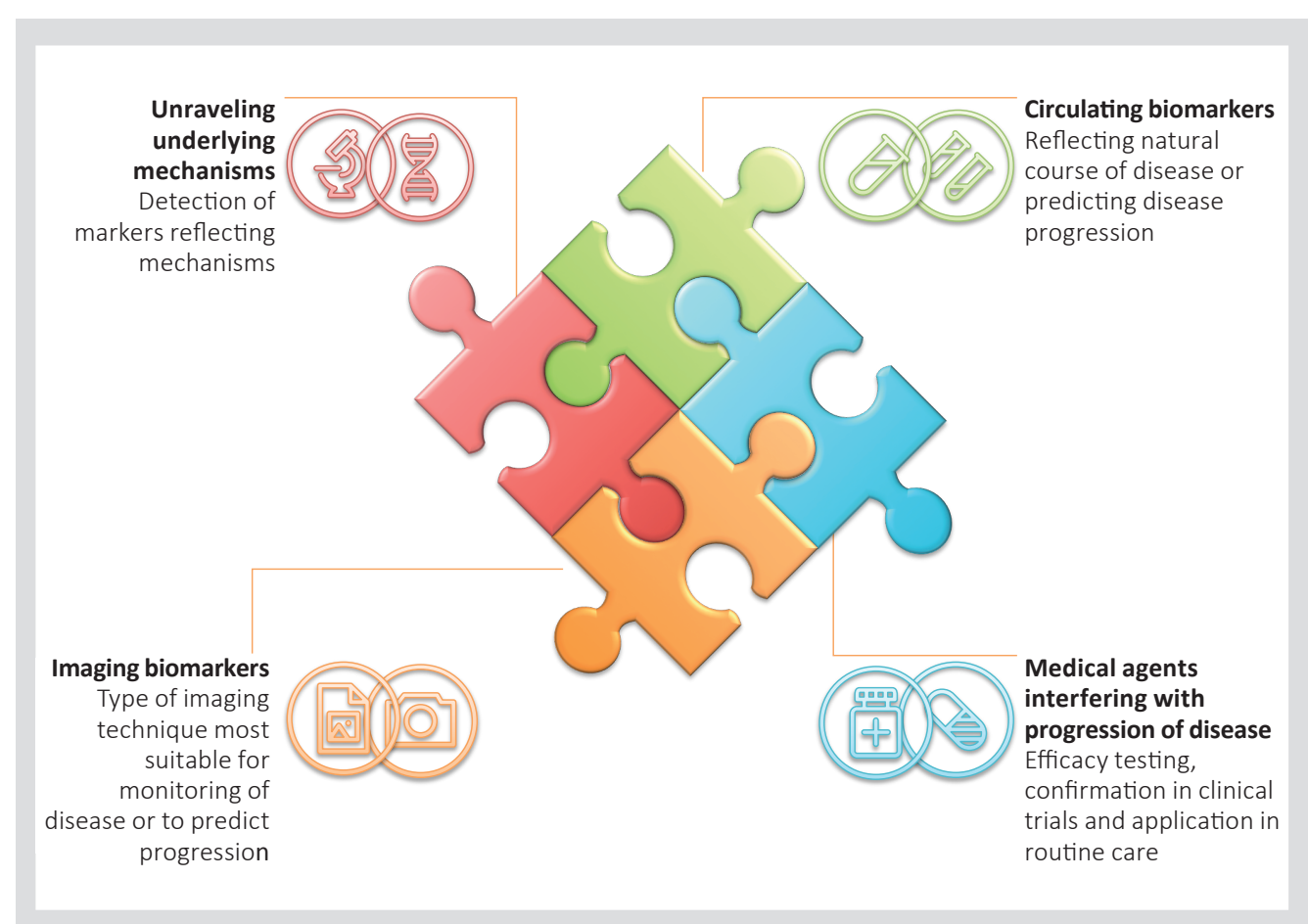

Figure 1. Domains and stages of research in aortic valve stenosis. Reflects part of the spectrum of translational research: from unraveling new mechanistic insights to application in the clinic by means of imaging techniques and circulating biomarkers reflecting the course of disease or predicting disease progression. Finally, investigation of potential medical treatments interfering with disease progression 
REFERENCES

Peeters F, Meex SJR, Dweck MR et al. Calcific aortic valve
stenosis: Rajamannan NM, Subramaniam M, Rickard D et al. Human
stenose in the heart: A biomolecular
aortic valve calcification is associated with an osteoblast stenosis: hard disease in the heart: A biomolecular approach towards diagnosis and
heart journal 2018;39:2618-2624

Aikawa E, Otto CM. Look more closely at the valve: imagin calcific aortic valve disease. Circulation 2012;125:9-11.

Dweck MR, Jones C, Joshi NV et al. Assessment of valvular tomography in patients with aortic stenosis. Circulation 2012;125:76-86.

4. Kim KM. Calcification of matrix vesicles in human aortic valve and aortic media. Federation proceeding

New SE, Aikawa E. Molecular imaging insights into early tion. Circulation research 2011:108:1381-91.

6. Olsson M, Thyberg J, Nilsson J. Presence of oxidized low density lipoprotein in nonrheumatic stenotic aortic valves. Arteriosclerosis, thrombosis, and vascular biology 1999; 19:1218-22.

Otto CM, Kuusisto J, Reichenbach DD, Gown AM, O'Brien valyular aortic stenosis. Histological and immunohistochemical studies. Circulation 1994;90:844-53.

8. Proudfoot D, Skepper JN, Hegyi L, Bennett MR, Shanahan CM, Weissberg PL. Apoptosis regulates human vascular calcation by apoptotic bodies. Circulation research

9. Rajamannan NM. Mechanisms of aortic valve calcification: the LDL-density-radius theory: a translation from cell signand circulatory physiology 2010;298: H5-15.

10. Shanahan CM. Inflammation ushers in calcification: a cycle 27 EI Accaoui RN, Gould ST, Haij GP et al. Aortic valve sclerose

in mice deficient in endothelial nitric oxide synthase. American journal of physiology Heart and circulatory physiology 2014;306:H1302-13

12. Mohler ER, 3rd, Gannon F, Reynolds C, Zimmerman R, Keane MG, Kaplan FS. Bone formation and inflammation in

13. Pawade $\mathrm{T}$, Clavel MA, Tribouilloy $\mathrm{C}$ et al. Computed TomogStenosis. Circulation Cardiovascular imagin 2018;11:e007146.

14. Peltonen T, Napankangas J, Ohtonen $P$ et al. (Pro)renin receptors and angiotensin converting enzyme $2 /$ angiotenAtherosclerosis 2011;216:35-43.

15. Gomez-Stallons MV, Wirrig-Schwendeman EE, Hassel KR, Conway SJ, Yutzey KE. Bone Morphogenetic Protein Signaling Is Required for Aortic Valve Calcification. Arteriosclero-
sis, thrombosis, and vascular biology 2016;36:1398-405.

16. Kaden JJ, Bickel haupt $\mathrm{S}$, Grobholz $\mathrm{R}$ et at. Receptor activ tor of nuclear factor kappaB ligand and osteoprotegerin regulate aortic valve calcification.
cellular cardiology 2004:36:57-66.

17. Nigam V, Srivastava D. Notch1 represses osteogenic pathways in aortic valve cells. Journal of molecular and cellular

18. Pawade TA, Newby DE, Dweck MR. Calcification in Aortic Stenosis: The Skeleton Key. Journal of the American College of Cardiology 2015;66:561-77. aortic valve calcification is associated
phenotype. Circulation 2003;107:2181-4.

. Yang X, Fullerton DA, Su X, Ao L, Cleveland JC, Jr., Meng X. Pro-osteogenic phenotype of human aortic valve intersttial cells is associated with higher levels of Toll-like recepCardiology 2009:53:491-500.

Yang $X$, Meng $X$, su $X$ et al. Bone morphogenic protein 2 induces Run $\times 2$ and osteopontin expression in human aortic valve interstitial cells: role of Smad1 and extracellular signal-regulated kinase $1 / 2$. The Journal of thoracic and cardiovascular surgery 2009,138.1008-15.

政 switch to control ectopic mineralization. Trends in molecular medicine 2013;19:217-26.

Luo G, Ducy P, McKee MD et al. Spontaneous calcification of arteries and cartilage in mice lacking matrix GLA protei

PA Fas SA, Williamson MK. Warfarin causes rapid calcification of the elastic lamellae in rat arteries and heart 1998;18:1400-7.

D. Society 2011:133:18406-12

Price PA, Urist MR, Otaw

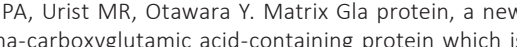
associated with the organic matrix of bone. Biochemica and biophysical research communications 1983;117:765-71 Chergers LJ, Cranerburg EC, Vermeer C. Matrix Gla-probosis and haemostasis 2008:100:593-603.

Schurgers $L$, Spronk HM, Skepper JN et al. Post-transla tional modifications regulate matrix Gla protein function: importance for inhibition of vascular smooth muscle cell calcification. Journal of thrombosis and

G. Venardos N, Bennett D, Weyant MJ, Reece TB, Meng X,
Fullerton DA. Matrix Gla protein regulates calcification of . The 2015;199:1-6.

. Cranenburg EC, Vermeer C, Koos R et al. The circulating nactive form of matrix Gla Protein (ucMGP) as a biomarker for cardiovascular calcification. Journal of vascular

C, Bultmann B, Janzen lar disease? Blood 2004;104:3231-2. (MGP) and bone morphogenetic protein-2 in aortic calcified lesions of aging rats. Jou stasis : ITH 2003;1:178-85,

Do AS, Hopman WM, Zimmerman D Garland JS, Morton AR. Warfarin and aortic valve calcification in hemodis
2007;20:417-22.

. Koos R, Mahnken AH, Muhlenbruch G et al. Relation of ora anticoagulation to cardiac valuvalar and coronary calcium American journal of cardiology 2005:96:747-9. tors 2 and 4 and enhanced expression of bone morphoge ectopic calcification by a direct interaction with
35. Caluwe R, Pyfferoen L, De Boeck K, De Vriese AS. The 51. Clark-Greuel JN, Connolly JM, Sorichillo E et al. Transform effects of vitamin K supplementation and vitamin Kantagodomized costrolled trials. Clinical kidney joun 2016;9:273-9.

36. Rattazzi $M$, Faggin E, Bertacco E et al. Warfarin, but not ivaroxaban, promotes the calcification of the aortic valve in ApoE-\% mice. Cardiovascular thercher

2018,36.e12438.

37. Kruger $\mathrm{T}$, Oelenberg $\mathrm{S}$, Kaesler $\mathrm{N}$ et al. Warfarin induces 54 cardiovascular damage in mice. Arteriosc
sis, and vascular biology 2013;33:2618-24

38. McCabe KM, Booth SL, Fu X et al. Dietary vitamin K and therapeutic warfarin alter the susceptibility to vascular cacification in experimental chronic kidney disease. Kidne

39. Schurgers LI, Spronk HM, Soute BA, Schiffers PM, DeMey tocalcinosis by high intake of vitamin $\mathrm{K}$ in rats. Blood 2007; 109:2823-31.

40. Shea MK, O'Donnell CJ, Hoffmann U et al. Vitamin K supplementation and progression of coronary artery calcium in older men and women. The American journal of clinica

41. Brandenburg VM, Reinartz $\mathrm{S}$, Kaesler $\mathrm{N}$ et al. Slower Progress of Aortic Valve Calcification With Vitamin K Supplementation: Results From a Prospective Interventiona

2. Peeters F, van Mourik MIW, Meex SIR et al. Bicuspid Aartic Valve Stenosis and the Effect of Vitamin K2 on Calcification phy/Magnetic ResonanceDesign. Nutrients 2018;10.

43. Beppu S, Suzuki S, Matsuda H, Ohmori F, Nagata S, Miyatake K. Rapidity of progression of aortic stenosis in patients with congenital bicuspid aortic valves. The American jour-

44. Roberts WC, Ko JM. Frequency by decades of unicuspid, bicuspid, and tricuspid aortic valves in adults having isolated aortic valve replacement for aortic stenosis, with or 2005;111:920-5.

45. Sun L, Chandra S, Sucosky P. Ex vivo evidence for the contrbution of hemodynamic shear stress abnormalities to the

. Schurgers LJ, Teunissen KJ, Hamulyak K, Knapen MH, Vik H, Vermeer $\mathrm{C}$. Vitamin $\mathrm{K}$-containing dietary supplements: menaquinone-7. Blood 2007;109:3279-83
ment

47. Dweck MR, Jenkins WS, Vesey AT et al. 18F-sodium fluoride uptake is a marker of active calcification and disease progression in patients with aortic

4. Irkle A, Vesey AT, Lewis DY et al. Identifying active vascular microcalcification by (18)F-sodium fluoride positron emis-

sion tomography. Nature communications 2015,6.7495.

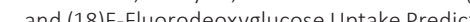
gression and Clinical Outcome in Patients With Aortic Stenosis. Journal of the American College of Cardiology 2015;66:1200-1.

50. Peeters F, Meex SJR, Dweck MR et al. Calcific aortic valve stenosis: hard disease in the heart. A biomolecular
Clartic cation: increased alkaline phosphatase and related events.

The Annals of thoracic surgery 2007:83:946-53.

Furukawa K. Recent advances in research on human aortic valve calcification

Fastic science and trans

A

al. Matrix Gla protein negatively regulates calcification of human aortic valve interstitial cells isolated from calcified aortic valves.
macological sciences 2018:136:257-265.

macological sciences 2018,136:257-265. ferences in Aortic Valva, Messika-Zeitoun D et al. Sex Diftector Computed Tomography in Aortic Stenosis. Circ-Cardiovasc Imag 2013;6:40-47.

Clavel MA, Messika-Zeitoun D, Pibarot P et al. The Complex Nature of Discordant Severe Calcified Aortic Valve Disease (a) Americancol

Clavel MA, Pibarot P, Messika-Zeitoun D et al. Impact of Aortic Valve Calcification, as Measured by MDCT, on Survival in Patients With Aortic Stenosis. Journal of
can College of Cardiology 2014:64:1202-1213

Hervault M, Clavel MA. Sex-related differences in calcific artic valve stenosis: pathophysiology, epidemiology, etiology, diagnosis, presentation, and outcomes. Structura

Baumgartner H, Falk V, Bax JJ et al. 2017 ESC/EACTS Guideines for the management of valvular $h$

Sean heart joun nal 2017,38.2739-2791. Sereled Discordance Severity of Aortic Stenosis: Is Valvular Fibrosis thodnamic crculation research 2017;120:681-691. sclerosis, and skeletal bone: is there a common link with $2013,34.1567-74$.

Chin CW, Shah AS, McAllister DA et al. High-sensitivity troponin I concentrations are a marker of an advanced hyperaortic stenosis. European heart journal 2014:35:2312-21. Henri C, Dulgheru R, Magne J et al. Impact of Serial B-Type Natriuretic Peptide Changes for Predicting Outcome in With Aortic Stenosis The Canadia journal of cardiology 2016;32:183-9.

Krau NC, Lunstedt NS, Freitag-Wolf Set al. Elevated growt pean journal of heart failure 2015;17:945-55.

Lancellotti P. Dulgheru R, Magne $t$ et al Elevated Plarma soluble ST2 Is Associated with Heart Failure Symptoms and Outcome in Aortic Stenosis. Plos one 2015;10:e0138940. Rosjo H, Andreassen J, Edvardsen T, Omland T. Prognostic usefulness of circulating high-sensitivity troponin $\mathrm{T}$ in aorcar diology 2011;108:88-91. (a)

proger $\mathrm{V}$, Nuyen $\mathrm{V}$, Robert T et al. Determinants and 6. 
69. Shen $M$, Tastet L, Bergler-Klein J, Pibarot P, Clavel MA. 84. Hiratzka LF, Creager MA, Isselbacher EM et al. Surgery for Blood, tissue and imaging biomarkers in calcific aortic valve Aortic Dilatation in Patients With Bicuspid Aortic Valves: A stenosis: past, present and future. Current

7. Lindman BR, Breyley JG, Schilling JD et al. Prognostic utility _ ical Practice Guidelines. Journal of the American College of of novel biomarkers of cardiovascular stress in patients with aortic stenosis undergoing valve replacement. Hear 8 2015;101:1382-8.

1. Fraser CG. Biological variation: from principles to practice.

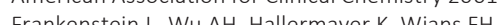
Cardiology 2016;67:724-731.

5. Verma S, Yanagawa B, Kalra S et al. Knowledge, attitudes, and practice patterns in surgical management of bicuspid aortopathy: a survey of 100 cardiac surgeons. The Journa nitsis $E$, Katus HA. Biological varia change value of high-sensitivity troponin $\mathrm{T}$ in healthy indviduals during short and intermediate follow-up periods.

ability of bar er ide and healthy controls.

Schindler El, Szymanski JJ, Hock KG, Geltman EM, Scott MG. Short-and Long-term Biologic Variability of Galectin-3 an Other Cardiac Biomarkers in Patients with Stable Heart Fal-

75. ure and Heathy Adults. Clinical chemistry 2016,62:360-6.

Four-Hour Biological Variation Profiles of Cardiac Troponi in Individuals with or without Chronic Kidney Disease. Clinical chemistry 2017;63:1655-1656.

76. Wu AH, Smith A. Biological variation of the natriuretic peptides and their role in monitoring patients with heart

Wu. AH, Wins $\mathrm{F}$.affe A. Biological 2004,6355-8

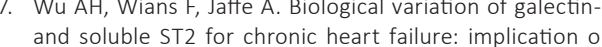
interpretation of test results. American heart journal 2013;165:995-9.

78. Della Corte A, Bancone C, Quarto C et al. Predictors of ascending aortic dilatation with bicuspid aortic valve: wide spectrum of disease expression. European journal of Association for Cardio-thoracic Surgery 2007:31:397-404; discussion 404-5.

79. Hahn RT, Roman MJ, Mogtader AH, Devereux RB. Association of aortic dilation with regurgitant, stenotic and func- 9 tionally normal bicuspid aortic valves. Journal of the Ame-

. Mn College of Das ogv 192, 19:283-8.

os JF et al. Natural hisminimally dysfunctional bicuspid aortic valye in the con munity. Circulation 2008;117:2776-84.

Krieger EV Hung $\perp$. Bicuspid aortic valve type: it takes two Heart 2018;104:544-545.

Della Corte A, Bancone C, Buonocore M et al. Pattern of ascending aortic dimensions predicts the growth rate of the aorta in patients with bicuspid aortic valve. JACC Ca

Kans IW, Song HG Yan, DH et al. Ass

bicuspid aortic valve phenotype and pacterion between dysfunction and bicuspid aortopathy: comprehensive evaluation using MDCT and echocardiography. JACC Cardiovascular imaging 2013;6:150-61.

9. Khoo C, Cheung C, Jue J. Patterns of aortic dilatation in American Society of Echocardiography : official pul of the of the American Society of Echocardiography 2013:26:600-5

0. Kim YG, Sun BJ, Park GM et al. Aortopathy and bicuspid aortic valve: haemodynamic burden is main contributor to aortic dilatation. Heart 2012;98:1822-7.
Schaefer BM, Lewin MB, Stout KK

Shaefer BM, Lewin MB, Stout KK et al. The bicuspid aortic valve: an integrated phenotypic classification of leaflet
morphology and aortic root shape. Heart 2008:94:1634-8 Peeters FE, Van der Linden N, Thomassen AL, Crijns HI, Meex SJ, Kietselaer BL. Clinical and echocardiographic determinants in bicuspid aortic dillatation: Results from longitudinal observational study. Medicine 2016;95:e5699. Ge Sara D, Me Aortic dilatation patterns and rates ondeau with bicuspid aortic valves: a comparative study with Marfan syndrome and degenerative aortopathy - Heart 2014;100:126-34.

. Holmes KW, Lehmann CU, Dalal D et al. Progressive dilation of the ascending aorta in children with isolated bicuspid aortic valve. The American journal of cardiolog

identify predictors of the $\mathrm{k}$ Ket al. Retrospective study to of aortic dilatation in patients with bicuspid aortic valves. Nature clinical practice Cardiovascular medicin

2008;5:821-8.
6. Evangelista A, Gallego P, Calvo-Iglesias F et al. Anatomical

81. Tzemos N, Therrien J, Yip J et al. Outcomes in aduls with and clinical predictors of valve dysfunction and aortic dilation in bicuspid aortic valve disease. Heart 2018;104:566-573. aorta with bicuspidone C, Dialetto $\mathrm{G}$ et al. The ascending with potential prognostic significance. European journal of cardio-thoracic surgery : official journal of the Europea Association for Guidelines. Circulation 2014;129:e521-643.

83. Hiratzka LF, Bakris GL, Beckman JA et al. 2010 ACCF/AH diagnosis and manascment of patients with The Disease: a report of the American College of Card dation/American Heart Association Task Force on Practice can College of Radiology, American Stroke Association, Society of Cardiovascular Anesthesiologists, Society for Cardiovascular Angrophy and Intersons, Society of Sterventiona cular Medicine Circultion 2010,121:e266 369. investigate the genetic basis of bicuspid aortic valve and its (Bicuspid Aortic Valve Consortium). Journal of the Amer- 
Summary

Nederlandse samenvatting

Valorization

Dankwoord

About the Author

List of publications 
mostly seen in patients $>65$ years of age. In patients with a congenital valvular malformation, the bicuspid aortic valve, AS is commonly seen at younger ages though. Once present, progression of hemodynamic severity is common but variable and medical therapies halting or reducing the progressive course of the disease are lacking. Therefore, physicians monitor progression of the disease by repeated echocardiographic examinations, and upon progression to severe (symptomatic) AS, patients are referred for valve replacement. Improvement of our understanding of the underlying mechanisms and pathways influencing AS and its progression is of uttermost importance to develop multi-biomarker strategies for monitoring and prediction and to discover targets amenable to medical therapy. My thesis presents several aspects in the translational field to gain insight into AS.

Firstly, I focus on current knowledge of pathways involved in AS and provide a rationale for the involvement of matrix Gla protein (MGP), an anti-calcification protein, in the pathophysiology through interaction with BMP-2 and hydroxyapatite crystals (Chapter 2). In the following chapters, we provide a step forward in the understanding of MGP involvement in AS. Herein, we show that the inactive (uncarboxylated) form of MGP (a vitamin K dependent protein, thus dependent on the presence of vitamin $\mathrm{K}$ for full bioactivity) is present in valve tissue of patients with AS in regions surrounding macrocalcification. Moreover, histological evidence that uncarboxylated MGP is present in areas without macrocalcification is provided, suggesting its involvement in the starting process of microcalcification (Chapter 6). Additionally, as a counter-mechanism, I showed that inhibition of vitamin $\mathrm{K}$ action through treatment with vitamin $\mathrm{K}$ antagonists (VKA) is associated with calcification in Chapter 3. In Chapter 4, I present the first pilot study on vitamin K1 supplementation, showing decreased inactive MGP (and increasing active MGP), and subsequent successful decrease in progression of the calcium score in patients with AS. The last step in MGP understanding is presented in Chapter 5, which outlines the trial design of our trial supplementing vitamin K2 in patients with bicuspid AS. This study aims to provide additional information by integrating an advanced technique, ${ }^{18} \mathrm{~F}$-sodium fluoride positron emission tomography, to show early effects of treatment with vitamin K2. More extensive studies are needed though to confirm the clinical impact of supplementation of vitamin $\mathrm{K}$ and with that, the impact of activated MGP on progression.

Secondly, imaging and circulating biomarkers aid in unraveling pathophysiology of AS and potential sex-related differences. In Chapter 7, we demonstrate differences in the dominant features of valvular tissue of males and females, being calcification in males and fibrosis in females. However, calcification activity, represented by ${ }^{18} \mathrm{~F}$-sodium fluoride uptake, showed variable differences between sexes according to measurement methods. Subsequently, in Chapter 8, we show a difference in the expression of circulating biomarkers between females and males. Again, fibrosis biomarkers were higher in women, whereas calcification biomarkers showed a higher expression in men. Thereby, these results give direction to sex-related differences in dominant mechanisms in AS and pose the question as to whether we should adapt amenable targets according to sex. Future studies will have to unravel these aspects.

Circulating biomarkers may also aid in the future to discriminate between progressive and stable disease. In Chapter 9, I investigated the natural variability of several circulating cardiac biomarkers, NT-proBNP, BNP, troponin-T, troponin-I and ST2, in a group of patients with AS. Although well-powered studies should be performed to confirm the role of single or combinations of these circulating biomarkers in prediction of progressive disease, we provide a basis for interpretation of serial measurements in AS for the first time. Additionally, an integrated multibiomarker approach including circulating and imaging biomarkers merits further investigation. 
In the last part of this thesis, I describe a patient group with bicuspid aortic valves and additional common vascular problem in this group; aortic dilatation and progression. Herein, it was found that clinical and echocardiographic parameters cannot predict progression of aortic dilatation by themselves to tailor follow-up strategies. These findings further support the concept of multi-biomarker approach with integration of hemodynamic parameters and genetics in BAV patients as well. The road to a tailor-made follow-up is still long. 
Aortaklepstenose (AS) is een complex ziektebeeld dat veel voorkomt, met name bij patiënten ouder dan 65 jaar. In patiënten met een aangeboren valvulaire malformatie, de bicuspide aortaklep wordt aortaklepstenose echter vaak gezien op jongere leeftijden. Als aortaklepstenose eenmaa aanwezig is laat het vaak een progressief beloop zien. Dit beloop is zeer variabel en tot op heden bestaan er geen medicamenteuze therapieën om het ziektebeeld te remmen of te stoppen. Om progressie te monitoren worden in de klinische praktijk op geregelde tijdstippen echocardiografische onderzoeken uitgevoerd. Bij progressie naar een ernstige (symptomatische) aortaklepstenose wordt de patiënt vervolgens doorverwezen voor een (minimaal invasieve) klepvervanging. Het is van belang om de kennis omtrent de onderliggende pathofysiologische mechanismen die AS (progressie) bepalen te verbeteren, om zo strategieën met verschillende biomarkers (uit bloedonderzoek) te ontwikkelen voor monitoring en predictie. Bovendien kunnen daarmee nieuwe medicamenten gemaakt worden die op deze markers ingrijpen. Dit proefschrift beschrijft diverse aspecten in het translationele spectrum van onderzoek om inzichten bij aortaklepstenose te verbeteren.

In het eerste gedeelte van het proefschrift beschrijf ik de huidige kennis van betrokken mechanismen in aortaklepstenose. Hierbij wordt ook de mogelijke rol van een vitamine $\mathrm{k}$ afhankelijk proteine, matrix gla proteine (MGP) onderbouwd, dat ingrijpt op de BMP-2 cascade en hydroxyapatite kristallen en zo in zowel de beginnende als gevorderde fase van AS een rol zou kunnen spelen (Hoofdstuk 2). MGP komt voor in de inactieve en actieve vorm, en heeft vitamine $K$ nodig om zijn functie uit te voeren. In de daaropvolgende hoofdstukken onderbouw ik de rol van MGP verder, door enerzijds aan te tonen dat er in klepweefsel niet alleen veel inactief MGP aanwezig is in de regio's rondom de verkalking, maar ook in regio's waar nog geen grove verkalking gezien wordt. Daarmee lijkt het een vroege marker van actieve verkalking, ook wel microverkalking genoemd (Hoofdstuk 6). Anderzijds laten we zien dat er een contra-mechanisme is; depletie van vitamine $\mathrm{K}$ door vitamine $\mathrm{K}$ antagonisten, en dus vermindering van de functie van MGP, is geassocieerd met verkalking (Hoofdstuk 3). Tenslotte volgen de eerste resultaten van een studie waarbij ik laat zien dat door vitamine K1 de verkalking van de klep in mindere mate verhoogd is ten opzichte van placebo, gemeten als calciumscore op computed tomography (CT). Parallel aan deze studie (beschreven in Hoofdstuk 4) loopt momenteel een studie die het effect van vitamine K2 bestudeert bij patiënten met een bicuspide aortaklep met aortaklepstenose door gebruik te maken van een geavanceerde techniek die calciumactiviteit in beeld kan brengen ("18F-natrium fluoride positron emission tomography" [PET]) (Hoofdstuk 5). In de toekomst zullen grotere studies moeten uitwijzen of het effect dat we zagen in Hoofdstuk 4 ook het beoogde klinische effect heeft; namelijk het vertragen van de aortaklepstenose progressie en de daarmee gepaard gaande complicaties

In het tweede gedeelte van dit proefschrift ga ik een stapje verder en onderzoeken we of er beeldvormende en circulerende biomarkers zijn die ons verder kunnen helpen om mechanismen en mogelijke geslachtsafhankelijke verschillen in aortaklepstenose te begrijpen. In Hoofdstuk 7 beschrijven we, door opnieuw gebruik te maken van de ${ }^{18} \mathrm{~F}$-natrium fluoride PET en CT scan, dat calciumactiviteit variabel is en bij vrouwen vaker sprake is van fibrose als dominant proces, terwif verkalking bij mannen het voorop staat. In Hoofdstuk 8 bevestigen we dit verschil op basis van verschillen die we vinden in expressie van circulerende biomarkers. Deze resultaten wijzen in de richting van geslachtsafhankelijke verschillen in dominante mechanismen, en stellen ons voor de vraag of toekomstige studies andere "geslachtsafhankelijke" targets voor interventies zouden moeten onderzoeken

Circulerende biomarkers kunnen ook van waarde zijn om progressieve ziekte van stabiele ziekte te onderscheiden. In Hoofdstuk 9 heb ik de natuurlijke variatie van verschillende biomarkers (NT-proBNP, BNP, troponine T, troponine I en ST2) onderzocht in patiënten met aortaklepstenose. 
Deze studie geeft voor het eerst een beeld van deze variatie bij AS dat van belang is voor de interpretatie van seriële metingen. Om de rol van enkele of combinaties van biomarkers te integreren in predictie van ziekte zullen grotere studies moeten komen die specifiek ingericht zijn om deze vraagstelling te beantwoorden.

In het laatste deel van het proefschrift (Hoofdstuk 10) wordt aandacht besteed aan patiënten met een bicuspide aortaklep en een ander probleem dat veel voor komt in deze populatie: aortadilatatie. In deze populatie bestudeerden we of we met echocardiografische en klinische parameters de snelheid van dilatatie kunnen voorspellen. Deze parameters lijken op zichzelf onvoldoende om individuele follow-up nauwkeurig te kunnen uitvoeren, maar ondersteunen wel het concept van een strategie met meer biomarkers die onder andere gericht zijn op hemodynamica en genetica.

Concluderend hebben de onderzoeken van dit proefschrift bijgedragen aan een verbetering in onze kennis van aortaklepstenose. De weg naar een geschikte therapie en factoren die het ziektebeloop kunnen voorspellen ligt nu open. 
This chapter discusses the future valorization of the findings presented in this thesis. Knowledge valorization is defined as the relevance of knowledge for social and/or economic purposes and to translate it into products, processes and innovations. ${ }^{1}$

\section{RELEVANCE}

Over the last decades, a progressive increase in valvular heart disease has been observed. Along with the occurrence and the growing elderly population, the necessity for regular follow-up, diagnostic tools and treatment costs have risen, and with that, the burden on our healthcare system. It has been described as the 'next cardiac epidemic'.,3 Within the spectrum of valvular heart disease, aortic valve stenosis is the most important type of valvular disease concerning clinical impact and mortality. It is responsible for approximately $45 \%$ of deaths in valvular heart disease. ${ }^{4}$ To date; no medical therapies have proven to effectively influence its natural course. Upon progression of aortic stenosis (AS), this leaves us with the only treatment possible; valve replacement, to which not all patients are suitable nor willing to undergo the intervention.

This thesis focuses on several aspects in AS, ranging from unraveling part of its pathophysiology and sex-related differences to the application of different circulating and imaging biomarkers to keep track of its course of progression. With that, it contributes to the optimization of follow-up strategies and specifies targets for intervention in this group of patients.

\section{VALORIZATION}

\section{TARGET GROUPS}

Findings in this thesis are relevant for patients with AS as well as cardiologists concerned with valvular heart disease. With a focus on knowledge regarding pathophysiology and target discover and possibilities to integrate a multibiomarker approach to monitor AS, these findings are relevan to the whole spectrum of patients with AS, ranging from beginning to end-stage and bicuspid or tricuspid aortic valves. Moreover, discovery of new targets might be relevant for organizations involved in the food industry and in drug development.

\section{PRODUCT}

Our findings resulted in new challenges and opportunities and trigger further research. For instance, the application of serial measurements of single or combinations of circulating biomarkers to monitor and predict progression requires additional research. A multibiomarker approach integrating imaging and circulating biomarkers would be the next step enabling personalized treatment options and timing of these interventions. Furthermore, others and we provide more and more evidence of the involvement of matrix gla protein (MGP) in AS progression. The first proof-of-concept trial showed that vitamin $\mathrm{K} 1$ had an effect on calcification already, and another ongoing trial treats patients with AS with a vitamin $\mathrm{K} 2$ supplement and applies innovative imaging techniques to monitor the potential effect. If trial results are positive, these findings may revolutionize preventive treatment of AS after further clinical evaluation.

\section{IMPLEMENTATION}

Studying mechanisms of AS led to a better understanding of the biomolecular mechanisms and opened new avenues for diagnosis and treatment. However, controversies and questions remain to be answered. As described in the general discussion, multiple steps and feedback-mechanisms 
in translational research are needed to transfer new understandings of AS mechanisms to mplement methods of diagnosis and treatment. With my research, we provided the first evidence that supplementation of vitamin $\mathrm{K}$ might be useful in reducing AS progression. However, large studies have to confirm this concept and reveal whether this effect results in actual clinica mprovement. Moreover, by investigating circulating biomarkers, the possibility to integrate my findings in a multi-biomarker strategy to monitor the course of the disease will have profound consequences in adequate timing of intervention. At last, this thesis (amongst other research) provides evidence that dominating processes in AS development and progression differ between males and females. These results may help to develop a personalized and more effective gender-specific approach to treatment. Multiple steps have been undertaken yielding new stepping-stones for future implementation research.

\section{REFERENCES}

Vandeberg. R. Waardevol: Indicator Coffey S, Cairns BJ, lung B. The modern epidemiology of heart valve disease. Heart 2016;102:75-85.

d'Arcy JL, Prendergast BD, Chambers JB, Ray SG, Bridgewa ter B. Valvular heart disease: the next cardiac epidemic. Heart 2011;97:91-3,

$B$, Williams MJ. Lack of progress in valvular ment era: increasing deaths and minimal change in mortaity rate over the past three decades. American heart journal 2014;167:562-567 e2. 
En dan zijn we aangekomen bij het dankwoord, waarschijnlijk het eerst gelezen hoofdstuk van dit proefschrift. Het schrijven ervan bleek niet zo makkelijk te zijn, en dat terwijl het altijd zo goed weg leest in andere proefschriften. Er zijn namelijk zoveel mensen die direct of indirect hebben meegewerkt aan het tot stand komen van dit proefschrift!

Allereerst wil ik mijn promotieteam bedanken; prof. dr. Harry Crijns, prof. dr. Leon Schurgers, dr. Bas Kietselaer en dr. Steven Meex.

Beste professor Crijns, beste Harry, u bent vanaf het allereerste begin van mijn onderzoek carrière betrokken geweest. Toen ik bij u kwam met de vraag of ik tussen mijn bachelor en master een jaar fulltime onderzoek mocht komen doen ontving $u$ mij met open armen in de wereld van AF en tachycardiomyopathie. Mijn enthousiasme voor onderzoek groeide in die tijd, en heeft ook zeker geholpen in mijn huidige PhD, ondanks dat het onderwerp totaal anders was; dat was tenslotte geen atriumfibrilleren. Ons maandelijks overleg kende vele gezichten; kort, lang, gestructureerd of ongestructureerd, maar vooral ook vaak verrassend. Uw helicopterview en vermogen om mogelijkheden te zien die niet voor de hand lagen zijn ongeëvenaard en ik ben $u$ dan ook erg dankbaar voor alle ideeën en mogelijkheden die u mij gaf. Daarnaast bent u ook wel de snelste met reacties na mijn reminders via Whatsapp.

Beste professor Leon Schurgers, beste Leon, je bent al sinds het begin van mijn promotie betrokken geweest bij verschillende projecten en ik ben dan ook erg blij dat je als laatste toegetreden bent tot mijn promotieteam. Ik zal ons eerste contact niet snel vergeten; tijdens een meeting voor het opzetten van de BASIK2 studie vousvoyeerde $i k j e$, waar je nogal verbaasd op reageerde. Het ijs was gebroken en vele gesprekken volgden bij jou op de universiteit, waar ik wel even moest wennen aan de informele structuur die aan de overkant van de brug heers en dus de eerste keren netjes buiten je kamer wachtte tot ik binnen mocht komen. Je enthousiasme voor onderzoek en je blik op potentiele klinische implicaties bewonder ik, alsmede je vakkennis en doorzettingsvermogen (met daarbij komend soms ook je ongeloof en ongeduld als iets bureaucratisch wat anders liep dan gepland). Onze overleggen waren niet zuiver gerelateerd aan werk; zaken in privé-sferen, vakanties en toekomstplannen werden uitgebreid en enthousiast besproken. Ik wil je heel hartelijk bedanken voor al je tijd die je hebt gestoken in mijn/onze onderzoeksprojecten (dat zijn er inmiddels toch behoorlijk wat), je prachtige verhalen maar ook voor de contacten waarmee je me kennis liet maken. Het diner samen met jou en Kathy Shanahan in München zal ik niet snel vergeten, net als de inaugurele rede die je afgelopen oktober hield. Ik hoop dat we in de toekomst nog vele redenen vinden om samen te blijven werken

Beste dr. Kietselaer, beste Bas, onze samenwerking begon tijdens mijn WESP, toen je me, zo bleek later, wel een erg optimistisch project had gegeven. Ondanks dat ik dit bij lange na niet af kreeg zag je dit niet als een gemiste kans, maar juist als een opstap naar een promotietraject. Voor dez kans ben ik je erg dankbaar. Mijn promotietraject begon met bicuspide aortaklep, en eindigde in een veel breder spectrum. De ideeën van jou en Steven aan het begin van mijn PhD hebben hiertoe geleid. Je hebt me nooit in de weg gestaan om mezelf te ontwikkelen; je gaf me vee verantwoordelijkheid en vrijheid, iets wat niet altijd vanzelfsprekend is. Door onze tegengestelde karakters (zo mag ik het wel noemen) heb je het denk ik niet altijd even makkelijk gehad met mij. Er was soms wat frictie en mijn ongeduld bij tijd en wijlen moest je op de koop toenemen. Het zorgde er ook voor dat er meer ideeën kwamen en ik mezelf enorm heb kunnen ontwikkelen. Ik waardeer je toegankelijkheid en bereikbaarheid, niet alleen tijdens het wekelijks overleg met 
Steven maar ook daarbuiten, en het gemak waarop jij met anderen kunt communiceren over zaken die eigenlijk helemaal niet zo makkelijk zijn. Bedankt!

Beste dr. Meex, beste Steven, bij de start van mijn promotie raakte jij als niet-cardioloog betrokken bij mijn promotietraject, iets waar ik je zeer dankbaar voor ben. Je liet me kennismaken op het lab met al zijn diversiteit en stelde onderzoeksvragen waar ik nog niet eerder over had nagedacht. Je scherpte, kennis en inzicht lieten me zien dat een promotietraject zoveel meer behelst dan data verzamelen en analyseren. Daarnaast was jij degene die me liet inzien dat we een balans moesten vinden tussen lopende en "zekere" projecten en nieuwe, risicovolle projecten om mijn promotietraject tot een goed einde te brengen. Bij het wekelijkse overleg samen met Bas was er altijd tijd genoeg om mijn verhaal te doen, maar ook daarbuiten had je een luisterend oor, wat ik als zeer prettig en vertrouwd heb ervaren. Tenslotte zal ik de leuke activiteiten en barbecues met de klinisch chemische-onderzoeksgroep niet snel vergeten; bedankt voor alles!

Beste prof. Dr. Marja van Dieijen-Visser, beste Marja, ondanks dat je officieel geen deel uitmaakt van mijn promotieteam wil ik ook jou graag bedanken. Voor de korte duur van officieel promotorschap was je inbreng, structuur en stabiliteit een groot voorbeeld voor me. Ik zie je werkwijze deels terug bij Steven, en dat kan ik alleen maar toejuichen. Je bent geïnteresseerd gebleven in mijn onderzoek en blijven meedenken, waarvoor ik je hartelijk wil bedanken.

Voorzitter en leden van de beoordelingscommissie, prof. Dr. Tilman Hackeng, prof. Dr. Michiel de Haan, prof. Dr. Arnoud van't Hof en prof. Dr. Tim Leiner, hartelijk dank voor de kritische beoordeling van mijn proefschrift. Furthermore, I would like to thank prof. Dr. David Newby for reading my thesis.

Dit proefschrift mag ik gaan verdedigen met twee mensen aan mijn zijde die, ieder op geheel eigen wijze, veel voor mij betekenen.

Beste Elton, AKA mister ACWAS, Dr. Dudnik, ik vergeet mijn eerste dag op onze kamer niet meer waar jouw directheid naar voren kwam doordat je je openlijk verbaasde dat er een werkplek mét computer voor mij was ingericht. Vanaf het begin van mijn promotie heb je mij geholpen (en me af en toe terug gefloten) om niet het wiel opnieuw uit te vinden als dat al lang gedaan was. Daarnaast zijn je kennis van statistiek en je bereidheid om samen projecten op te zetten en uit te werken een grote inspiratie voor me geweest. Ook onvergetelijk was ons project waarvoor we "even" CT-scans bij Philips in Eindhoven gingen uitwerken en al ons zure voorbereidende werk in zo'n 10 seconden ongedaan werd gemaakt. Dat was misschien niet de beste dag aangezien we beiden niet het karakter bezitten om dit naast ons neer te leggen, maar uiteindelijk hebben we het toch maar geflikt en zijn er nu al de nodige papers uit gekomen. Dank voor alle gezelligheid tijdens congressen, maar zeker door gewoonweg jezelf te zijn; ik heb je aanwezigheid (lees: slechte muzieksmaak, sarcastische opmerkingen, je uitgesproken reacties en je talent om bij iedereen vlaai te regelen) op de kamer gemist; hopelijk tot snel weer in de kliniek!

Lieve Cro, wij hebben elkaar leren kennen in het begin van onze studietijd bij Circumflex en ik mag wel zeggen dat er direct een klik was. In eerste instantie zo verschillend van elkaar, maar tegelijkertijd ook een hoop eigenschappen gemeen. We hebben een hoop mooie events meegemaak met de jaarclub, maar ook onze diners, borrels, feestjes en onze vakanties waren (en zijn) altijd top. Niet alleen voor de leuke dingen, maar ook voor serieuze en minder leuke gesprekken was je er toen ik in een wat onzekere periode zat. Ik kan altijd helemaal mezelf zijn bij je, en dat geeft een enorm vertrouwd en warm gevoel. Ik ben heel blij dat je sinds iets meer dan een jaar zo gelukkig met Pim (it's in the name) bent; dat we samen nog maar veel mogen meemaken! Overigens: hoe je het af en toe met mij volgehouden hebt (en nog steeds) als "Duracellkonijn" is me nog altijd een raadsel. Bedankt!

Tiidens mijn promotie heb ik een tweetal verhuizingen naar andere kamers mee mogen maken Daarbij gingen we van een 6-persoonskamer naar een 14-persoonskamer, wat leidde tot een enorme toename samenwerking en plezier met fijne collega's. Met nogal wat uiteenlopende persoonlijkheden en een scala aan taferelen die daar plaatsvonden denk ik terug aan een fijne tijd. Op alfabetische volgorde:

Arantxa, jij kwam bij ons broekies op de kamer terwijl je al bijna klaar was met je opleiding tot cardioloog en toen besloot om eerst te promoveren. Een succesverhaal als je het mij vraagt; je hebt het wereldje van onderzoek snel eigen gemaakt en daarnaast ook nog een prachtige zoon op de wereld gezet.

Bianca, de kamer jongste toen ik nog bij jullie op kamer zat. We hebben elkaar niet heel veel gezien omdat projecten langs elkaar liepen. Volgens mij heb je een goede start gemaakt met je promotieonderzoek en ik wens je dan ook heel veel succes met het vervolg.

Bouke, in beginsel zo rustig ogend, tot ik je beter leerde kennen. Je scherpzinnige opmerkingen maar ookvooral dewijzewaaropje de 4D-flowstudies opzettezijnalleen maarbewonderingswaardig te noemen. Ik twijfel er niet over dat jij binnenkort mooi proefschrift op de plank gaat leggen. Daarnaast kwam er met jouw komst op de kamer meteen een nieuw initiatief; de PhD-ski! Dank voor de twee prachtige midweken die ik met je mocht organiseren!

Floor, als PhD zat jii "ver" weg van ons op de universiteit. Dit weerhield je er niet van om mee te gaan op de uitjes, maar ook op skivakantie, waar je liet zien een echt skitalent te zijn! Succes met het afronden van je promotie.

Job, de stilste werker van ons. Je moest in het begin wennen aan alle chaos en persoonlijkheden, maar hebt je plekje helemaal gevonden. Je produceert papers alsof het werkelijk niets is (ook a krijg je wat ondersteuning in de analyses); wil je me het geheim eens vertellen?

Jort, AKA Jordan Brands, wij begonnen vrijwel tegelijkertijd aan ons promotietraject. Altijd duidelijk aanwezig, een mening over wat jij wel en niet hoefde te doen. Zelfs 19 versies van één protocol sloegen je niet uit het veld om door te zetten en grappen te blijven uithalen. Ik zie je nu in de kliniek als ik weer eens hulp nodig heb met interne geneeskunde. Hopelijk snel weer als directe collega. Succes met de verdediging van je proefschrift! Ga je me nog eens vertellen hoeveel paren sneakers je nu eigenlijk echt hebt?

Luuk, ik ken je eigenlijk vooral toen je Elton en mij hielp met het vergaren van de CT-data in Eindhoven; een proces dat niet al te soepel verliep helaas. Je hebt je gelukkig door dit voorbeeld niet laten ontmoedigen en bent ook begonnen aan je promotietraject; succes!

Manouk, mijn opvolgster voor de BASIK2-studie, en hoe! We leerden elkaar kennen op congres in Leipzig, waar jij een praatje mocht houden. Je enthousiasme voor onderzoek was duidelijk 
merkbaar, en ik ben dan ook heel blij dat je bent begonnen aan je promotieonderzoek bij Bas Bekkers en prof. Crijns. Je hebt een lieve en warme uitstraling, maar staat je mannetje. lets wat zeker niet vanzelfsprekend is bij je buurman: chapeau! Dank voor je inzet voor de BASIK2. Ik hoop dat je, nu je besloten heb om de huisartsenopleiding te gaan doen, nog betrokken blijft! Succes!

Mark, AKA shady hazy, niet de allergrootste, maar zeker wel een van de luidste (op Jort na)! Jouw inzichten in de wereld van het onderzoek waren verhelderend en je hebt me meer dan eens geholpen als ik weer eens vast liep. Dank daarvoor! Ook je enorme drive en doorzettingsvermogen bij feestjes en borrels zijn niet te evenaren en leverden meer dan eens hilarische momenten op!

Martijn, mister CARMENTA, eindelijk is het dan zover; na lang zwoegen is jouw studie afgerond. Ik hoop binnenkort je proefschrift te zien. Dank voor je gezelligheid en dank voor je inzet om me te eren hoe de CT-coronairen te beoordelen. Ook zeker bedankt voor het aanwakkeren van mijn enthousiasme om beter te worden in wielrennen toen je me voor een "kort en niet te zwaar" (70km met in de eerste $30 \mathrm{~km} 700$ hoogtemeters) ritje meenam in het heuvelland en me zo ongeveer elke heuvel naar boven praatte!

Masih, naast Mark, Elton en Jort zat jij ook op de 6-persoonskamer op de stafgang, die doo mannen gedomineerd werd. Elke dag met jou was weer een verrassing; je kende hoge pieken maar ook diepe dalen. De grap die je met Mark uithaalde kan ik me nog als de dag van gisteren herinneren, maar ook de dagen dat je alleen maar op je knieën op je bureaustoel kon zitten! Dank voor je uitleg en geduld bij het ontwerpen van figuren voor mijn papers!

Michiel, met jouw komst kwam er een vrolijke noot in de kamer! Ik ken maar weinig mensen die promoveren en elke dag met een lach binnen komen. Je bent een harde werker en vooral een ontzettend vroege vogel, die in de uurtjes dat Jort nog niet functioneert het fort bewaakt. Heel veel succes met de rest van je promotie.

Mindy, mijn buurvrouw in de grote kamer. Ook jij steekt je mening niet onder stoelen of banken wat af en toe tot hilarische gesprekken/en confrontaties leidde met niet nader te noemen personen. Je besloot om je carrière na je promotie voort te zetten in de cardiothoracale chirurgie, dus wellicht komen we elkaar in de toekomst nog tegen. Succes!

Nick, de snelheidsduivel op de motor. Je hebt het niet altijd even makkelijk gehad tijdens je promotie met alle klinische onderzoeken die op je bord lagen. Toch heb je je er met opgeheven hoofd doorheen geslagen en ik ben ervan overtuigd dat we binnenkort een mooi proefschrift van je kunnen verwachten! We zullen binnenkort directe collega's worden als je aan je opleiding begint in het MUMC+; heel veel succes!

Nikki, als opvolger voor de ACWAS had Elton zich geen betere kunnen wensen. Je tomeloze inzet en bereidheid om het hele land door te reizen zijn zeker niet onopgemerkt. De inclusie van de ACWAS is inmiddels klaar, en dus kan nu een andere fase beginnen; het analyseren en opschrijven! Je bent een serieuze en harde werker, wat ik alleen maar kan aanmoedigen. Denk je wel af en toe aan jezelf?

Ömer, AKA Ömi, toen jij begon aan je promotie kwam je als $5^{\text {de }}$ man op de kamer van 6. Verreweg de meest fatsoenlijke man van de kamer, die zelfs voor mij opkwam als er weer eens wat vrouwonvriendelijke praat was. Daarnaast altijd "the last-one-standing" in de kroeg. Je staat je mannetje wel, en ondanks dat ik er niet heel veel over gehoord heb, ben ik ervan overtuigd dat ook jouw promotie binnenkort tot een goed einde gebracht zal worden. Succes in Heerlen en hopelijk tot snel!

Sema, door je aanwezigheid op andere werkplekken hebben we elkaar niet veel gezien, ondanks dat je mijn buurvrouw was op de 6-persoonskamer. Inmiddels alweer een tijd in Heerlen aan het werk; succes met je opleiding!

Sibel, onze interesse voor het onderzoek en beoordeling van CT-coronairen brachten ons vaak bi elkaar, ondanks dat jij op de universiteit zat en dus eigenlijk "ver weg". Ik ken geen vriendelijker persoon dan jij! Altijd behulpzaam, tijd voor uitleg en tijd om te brainstormen. Dat is echte een enorme kracht. Verlies die niet, en laten we nog eens samen op congres gaan! Succes met je opleiding en de laatste lootjes voor je proefschrift.

Theo, je was je promotie al bijna aan het afronden toen ik begon. Je nuchtere invalshoek en het gemak waarop alles je af lijkt te gaan zijn me bijgebleven, maar ook je enthousiasme om biertjes te drinken in de stad samen met Martijn en mij.

Twan, ik ken oprecht niemand die zo goed kan praten als jij! Ook jij begon aan je promotieonderzoek terwiil je bijna klaar was met je opleiding maar koos ervoor om niet op de onderzoekerskamer te gaan zitten. Wijs besluit denk ik. Toch ben ik blij dat we je af en toe nog mochten helpen met het opzetten van statistiek en figuren. Je proefschrift was binnen no-time geschreven; respect!

Yvonne, de laatste van de onderzoekersgroep, maar zeker niet de minste. Vanaf het begin van je promotie was je in voor alles; vol goede moed ging je er tegenaan. Daarmee had ik een goede "partner-in-crime" voor de organisatie van menig uitje en borrel, waarvoor dank! Helaas leerde ook jij dat promoveren met hoge pieken, maar ook diepe dalen gaat. Je slaat je goed er doorheen ook al is het niet altijd makkelijk! Houd de spirit vast, en blijf vooral jezelf; jij komt er zeker! Ook ji zult binnenkort met je opleiding starten; gefeliciteerd en heel veel succes!

Een speciaal woordje voor de promovendi van het CDL. Dorien, zonder jouw kennis van statistiek en je bereidheid me te helpen met zowel makkelijke als moeilijke vraagstukken had ik lang niet alles zo mooi kunnen analyseren en opschrijven (en daadwerkelijk begrijpen!). Daarnaast werd ik door je verhalen over de marathon geïnspireerd om er zelf ook 2 te lopen. Ik hoop dat je knie na die ultramarathon (respect!) nog hersteld, zodat je weer vol goede moed een nieuwe marathon kunt uitzoeken. Noreen, ook jij bedankt voor je hulp en de nodige statistische kennis bij het schrijven van mijn eerste stuk! Het ga je goed verder! Judith, bedankt voor je heldere uitleg van biologische variatie. Heel veel succes met je opleiding in Rotterdam! Lieke, Wim, William en Stefanie, dank voor jullie gezelligheid en bedankt voor de tijd die jullie namen om altijd een praatje te maken als ik langs kwam op de vijfde etage.

Naast miin kamergenoten en directe PhD-collega's wil ik ook nog graag een klein woordje van dank uitspreken aan de personen die op de PhD-kamer zaten toen ik een jaartje als groentje onderzoek kwam doen tussen mijn bachelor en master; Sandra, Bob, Ron, en lone. Ik werd warm ontvangen op de kamer, ondanks dat ik echt he-le-maal niets wist van onderzoek en jullie meer heb lastig gevallen met vragen dan wie dan ook. Jullie manier van (samen)werken was een inspiratie voor me. Bob, een 
extra bedankje voor jou; dank voor je tijd om mij eindeloos uitleg te geven over AF, de poli en je onderzoek. Ik waardeer het dat je me hierin hebt betrokken, zodat ik al vroeg een publicatie op mijn CV kon zetten!

Ook wil ik graag alle cardiologen van het MUMC+ bedanken voor de medewerking bij inclusies voor de diverse studies die gedurende mijn promotie draaiden. Verder wil ik graag de cardiologen van het Zuyderland en het Laurentius ziekenhuis bedanken voor hun waardevolle bijdrage aan de inclusie van patiënten voor de BASIK2 studie.

Beste dames van cardioresearch; zonder jullie ondersteuning was er nooit een kaft om dit proefschrift gekomen. Dank voor jullie inzet bij het inplannen van patiënten, de hulp als een venapunctie niet lukte en het geduld als er weer eens iets opnieuw ingepland moest worden. Ik zie jullie momenteel iets minder, maar doe mijn best voor de inclusies van de trials nu ik in de kliniek sta.

De echokamer verdient ook een plekje in dit dankwoord; zonder jullie medewerking had ik heel wat meer moeite gehad om de resultaten bij elkaar te krijgen van dit proefschrift. Alissa, super bedankt voor je tomeloze inzet bij de beoordeling van alle echo's voor het bicuspidie en aortadilatatie project!

De secretaresses, maar met name Miriam Habex-Froidmont en Saskia Buskes. Miriam, bedankt voor alle planning en organisatie van afspraken, die je menig grijze haar hebben bezorgd. Daarnaast zal ik de gesprekken die we hebben gevoerd niet vergeten. Je werkt helaas niet meer bij de cardiologie, maar de weg naar de andere stafgang is me wel bekend. Saskia, bedankt voor de organisatie rondom mijn boekje en promotie. Zonder je inzet had dit best wel eens een langdurig traject kunnen worden.

Dit proefschrift was eveneens nooit tot stand gekomen zonder goede samenwerking met vele andere specialismen.

Allereerst het CDL, waar ik altijd met open armen ontvangen werd en er altijd tijd en ruimte was voo een brainstorm of overleg. Vincent Kleijnen, bedankt voor al je hulp bij het verwerken en analyseren van de monsters van de biologische variatie en de CATApULT. Dit was een tijdrovende klus die op het laatste moment, onder nogal wat tijdsdruk, foutloos uitgevoerd moest worden. Ook wil ik graag Otto Bekers, Alma Mingels en Petal Wijnen bedanken voor de fijne samenwerking. Tenslotte wil ik de analisten van het laboratorium bedanken voor hun medewerking en hulp als ik samples kwam afdraaien en analyseren. Zeker als dat weer eens in het weekend moest gebeuren!

De afdeling radiologie, in het bijzonder professor Joachim Wildberger, Suzanne Gerretsen, Suzanne Gommers en Casper Mihl, voor de zeer waardevolle bijdrage aan mijn lering en kennis omtrent beoordeling van CT-coronairen en de constructieve feedback en hulp bij diverse onderzoeksprojecten. In een proefschrift waar beeldvorming een centrale rol heeft is jullie hulp en kennis onontbeerlijk.

Professor Wildberger, graag bedank ik u ook voor de tijd die u nam om advocaat van de duivel te spelen bij diverse manuscripten die ik schreef; deze werden telkens een niveau hoger getild!

Jan Bucerius, bedankt voor je hulp en betrokkenheid bij de PET in de BASIK2 studie. Natuurlijk wil ik ook de laboranten van de CT bedanken voor hun inzet.

Een groot woord van dank voor Eslina, Kim, llse en Anouk voor alle avonduren waarin jullie PET/MR scans maakten (en maken) voor de BASIK2 studie!
Ook een woord van dank aan de cardiothoracale chirurgen CTC-assistenten en niet te vergeten de verpleegkundigen op afdeling D4, voor alle inzet bij de inclusie en het bewaren van klepmateriaal voor de CATAPuLT studie. Ondanks dat we nog druk bezig zijn met de analyses en er slechts een klein manuscript in dit proefschrift beschreven wordt, verwacht ik dat er nog mooie resultaten verkregen worden met al het materiaal dat we met jullie hulp hebben kunnen onderzoeken!

Voor de andere afdelingen gaan we de brug over naar de universiteit; allereerst de afdeling biochemie. Armand, bedankt voor de tijd en de hoeveelheid werk die je gestoken hebt voor de verwerking van het kleppen van de CATAPuLT studie, ook al was dat soms niet zo eenvoudig te plannen! Daarnaast was je nooit te beroerd om met Leon en mij mee te denken over zinvolle analyses. Ik denk dat we nu al aardig wat uit deze studie hebben gehaald. Heel veel succes met de laatste lootjes voor je eigen proefschrift! Rick, alhoewel we geen directe projecten samen hebben gedaan ook voor jou een bedankje voor het kritisch meedenken, maar ook met de praktisch uitvoering van analyses. Ook jij veel succes met je proefschrift. Ik ben benieuwd wie van jullie de eerste is; of gaan jullie er een duo-promotie van maken? Petra en Cecile, zonder jullie was ik nergens geweest voor de uitvoering van de kleuringen; hartelijk dank voor jullie harde werk, flexibiliteit en de wijze waarop jullie mij op eenvoudige wijze uitlegden hoe het allemaal écht werkte!

Ook de Biobank, en speciaal Claudia Bosma en Corine Coorens, wil ik bedanken voor de samenwerking. Als de Biobank niet zo ontzettend netjes georganiseerd was geweest had ik niet geweten hoe alle samples van alle studies netjes had moeten opslaan.

Marc Dweck, I would like to thank you for giving me the opportunity to come to Edinburgh for fellowship in the last year of my PhD. I received a warm welcome from your group; I learned such a lot about PET/CT and NaF! Hopefully our project will lead to a publication in the end of course, but even more, I hope to stay in touch and hope to visit Edinburgh another time for a collaboration (or extension). Professor David Newby, thank you for having me in Edinburgh, and for the great dinner I got to attend while there!

Mhairi, Russell, Nick and Jack, thank you all for the help and also for the great company during dinners, drinks and the half marathon of course! (Russel, send Emily my best!) Jacek, thank you for your enthusiasm in teaching me the essentials of Fusionquant; love working with that program.

Maryam en Mahboobeh, dank voor jullie enthousiasme toen ik jullie vroeg om het design van mijn proefschrift te verzorgen. Ook bedankt voor het geduld dat jullie hadden toen ik maar bleef twijfelen over kleurstellingen van de omslag en jullie een behoorlijk aantal versies liet maken (om vervolgens bij een van de eerste versies terug te keren). Jullie ideeën waren heel vernieuwend voor mij en laat echt zien hoe vakkundig jullie zijn. Jullie hebben een prachtig design hebben gemaakt. Bedankt!

Ook buiten het ziekenhuis zijn er vele personen die op geheel eigen wijze een bijdrage hebben geleverd aan dit proefschrift. Niet alleen door interesse te tonen in mijn werk (ook al was dat soms moeilijk uit te leggen), maar vooral ook om even helemaal af te kunnen schakelen. Zo wil ik de "Vriavbo" leden van het dispuut bedanken voor het plezier tijdens alle borrels en events die we samen mee hebben gemaakt; dat er nog maar vele mogen volgen! Daarnaast ook een woordje van dank voor de jaarclub; onze band is hechter geworden dan we ooit hadden kunnen hopen. Onze weekendjes en uitjes staan altijd 6 maanden lang geblokkeerd in mijn agenda. Op naar de tweede 
lustrumvakantie! Ook de dames van het hockeyteam wil ik bedanken voor het mooie team dat we samen vormen. Het is heerlijk om met jullie samen te spelen op zondag (ook al klaag ik wel eens over de mid-mid positie waar ik liever wat minder vaak zou staan) en alle initiatieven die jullie ondernemen buiten het veld. Voor mij een goed moment om even af te schakelen!

Sabine en Jaap, ook jullie verdienen een speciaal plekje in dit dankwoord. Jullie waren altijd supe geïnteresseerd in mijn promotie en de voortgang ervan. Daarnaast wil ik jullie ook graag bedanken voor alle leuke borrels, etentjes en de festivals die we samen bezochten (altijd een succes). Laten we binnenkort eens samen bedenken welk sterrenrestaurant we samen kunnen aandoen, want ik geloof dat dat het enige is waar we nog niet over uit waren.

Lisanne, ook jou wil ik heel erg bedanken voor al je lieve berichtjes en de fijne tijden die we samen hebben. Je bent zo heerlijk jezelf en laat je door niemand daar vanaf brengen. Laten we probere om dit jaar weer eens daadwerkelijk een weekend weg te plannen tussen de diensten door!

Corine, ook jou zal ik zeker niet vergeten in dit dankwoord. Dank voor het klankbord dat je me bood, maar vooral voor je geheel eigen wijze om altijd gelegenheid te creëren voor gezellige avonden/dagen/weekendjes! Nu je EINDELIJK weer in Den Bosch woont hoeven we daar in ieder geval niet meer dat roteind voor te reizen:) Natuurlijk ook een dank voor Lois en Marloes voor de heerlijke themadiners. De vorige heb ik gemist; wanneer is het tijd voor een nieuwe?

Na deze al ontzettend lange lijst kom ik langzaam richting het eind van mijn dankwoord, maar niet zonder een aantal heel speciale personen te noemen.

Lieve Tom en Neli, ik kom nu al ruim 9.5 jaar bij jullie over de vloer en heb me vanaf het begin af aan heel welkom gevoeld (ondanks de vuurdoop die ik kreeg met de traditionele nieuwjaarsborrel in Weert waar ik meteen de hele familie mocht ontmoeten). We hebben al veel leuke, maar ook moeilijke dingen samen meegemaakt. Jullie voorbereiding van elk diner en borrel is ongeëvenaard; dat er nog maar velen mogen volgen!

Lieve Laura en Mark, ook jullie horen zeker in dit riitje thuis. Ik geloof dat ik jullie voor het eerst in de kroeg ( $C^{\prime}$ est la Vie) heb ontmoet toen ik met Pim was. Ik ken geen enkel koppel dat in de afgelopen jaren zoveel verbouwd heeft, respect daarvoor hoor. Ik voel me altijd welkom bij jullie en kom dan ook graag van je kookkunsten genieten Laura. Dank ook voor de vakinhoudelijke discussies; een goede uitlaatklep voor mij die af en toe wel nodig was/is.

Lieve Cathelyn, Bram en natuurlijk Dex! Mijn kleine sissie, zo groot en een prachtige zoon gekregen. Ik geloof dat we vooral mam vroeger wel meer dan eens tot waanzin hebben gedreven met onze waanzinnige botsingen waar jij witheet werd en ik ijskoud. Onze leventjes zijn zo anders, maar in tegenstelling tot vroeger kunnen we nu goed met elkaar praten en kom ik graag bij jullie over de vloer. Ik wens jullie ook het beste, al zit ik er niet over in dat dat wel goed komt.

Lieve Alexander, Alex, mijn kleine broertje. Door onze 12 jaar leeftijdsverschil kan ik me nog goed herinneren hoe je was als baby; een charmeurtje! Die charmes niet verloren, maar inmiddels de grootste van ons drieën en druk bezig met je studie BMW. Ik ben heel benieuwd wat de toekomst je verder gaat brengen. Vergeet niet van je studententijd te genieten; best time of your life! ieve pap en mam, jullie hebben in mijn jeugd ervoor gezorgd dat ik alles had wat mijn hartje begeerde en me daardoor volledig op mijn toekomst kon storten. Hierdoor ben ik geworden wie ik nu ben, en daar ben ik jullie eeuwig dankbaar voor. We hebben het niet altijd even makkelijk gehad en jullie hebben altijd heel hard gewerkt om alles wat er is voor elkaar te boksen. For better and for worse, ook in moeilijke tijden zijn jullie er met een luisterend oor en met praktische tips. Jullie staan onvoorwaardelijk voor ons klaar, en weet dat dit ook wederzijds is!

En dan... save the best for last, lieve Pim! Wat moet ik toch zonder jou. We zijn nu al 9.5 jaar samen, en nog steeds verveel ik me geen dag met je. Je staat met beide benen stevig op de grond, bent een alfa-mannetje als het nodig is, maar stiekem vooral heel eerlijk, recht door zee, lief en zorgzaam. We hebben al zoveel meegemaakt dat ik er een hoofdstuk vol over zou kunnen schrijven (zal ik je besparen;)). Onze buitenlandavonturen in het begin van onze relatie, waar we elkaar welgeteld 2 maanden zagen in 1 jaar door diverse stages in Zuid-Afrika, India en New York, waren een goed test voor onze relatie, die vanaf het begin goed was en alleen maar sterker is geworden. Daarna hebben we voornamelijk samen gereisd naar toch een behoorlijk aantal mooie oorden, maar ook onze trip naar Berlijn, waar we samen de marathon finishte in 3:59:13 (beiden;)) zal ik nooit meer vergeten. Waar gaan we dit jaar naartoe? Naast je drukke baan, alle sport- en sociale activiteiten zorg je ervoor dat ik af en toe mijn rust bewaar, iets waar ik nooit erg goed in ben geweest. Hoe ji dat doet is me nog altijd een raadsel, maar still trying to learn, dus misschien komt dat nog ooit. Zonder jouw steun was er denk ik nooit een boekje gekomen, want het ging niet altijd even makkelijk de afgelopen periode. Ik zou niet weten wat ik zonder je zou moeten. Ik hou van je schatje! 


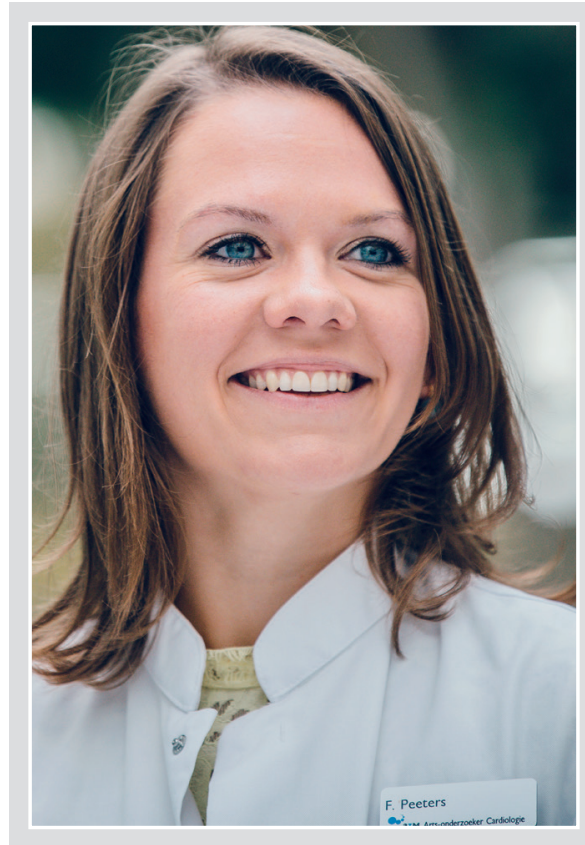

Frederique Peeters

November $10^{\text {th }} 1987$ in Venlo, the Netherlands

\section{ABOUT THE AUTHOR}

She attended pre-university education at Bisschoppelijk College Broekhin (Roermond, the Netherlands) and graduated in 2006 for both the Atheneum (dual language) and the Internationa Baccalaureate (IB). That year, she started studying Molecular Life Sciences at Maastricht University (Maastricht, the Netherlands) and successfully completed the first year. In 2007, Frederique started her medical training at the Faculty of Health Medicine and Life Sciences at Maastricht University (Maastricht, the Netherlands) and studied 1.5 months in Italy in her second year (Ferrara, Italy). After obtaining her Bachelor's degree, she decided to postpone the start of her Masters degree and applied for a fulltime function as research student to participate in research studying atrial fibrillation and tachycardiomyopathy under supervision of Prof. Dr. Harry J.G.M. Crijns at the department of Cardiology. She proceeded her medical training in 2011 and spend three periods of internships abroad (Cape Town, South-Africa; Manipal, India and Antwerpen, Belgium). In her final year of medical training she participated in a research internship focusing on the bicuspid aortic valve at the department of Cardiology of Maastricht University under supervision of Dr. Bas L.J.H. Kietselaer. Following this period, she did a clinical internship in Cardiology in Maxima Medical Center (Veldhoven, the Netherlands) under supervision of Dr. R. Verbunt. Frederique graduated as a medical docter at Maastricht University in 2014 and started her PhD-program in the field of aortic valve disease under supervision of Prof. Dr. Harry J.G.M. Crijns, Prof. Dr. Leon J. Schurgers, Dr. Bas L.J.H. Kietselaer and Dr. Steven J.R. Meex. As part of her PhD-program, she collaborated with different specialties within the hospital and university departments of Cardiothoracic surgery, Radiology and nuclear medicine, Clinical Chemistry and Biochemistry). She visited the Edinburgh Centre for Cardiovascular Science (Edinburgh, Scotland, United Kingdom) as a research fellow under supervision of Dr. Marc R. Dweck. Her scientific work was presented at various national and international congresses and patient-related conferences. Additionally, Frederique received a travel grant from the European Society of Cardiology. In September 2018 Frederique started her clinical training in Cardiology at the Maastricht University Medical Center+ (Maastricht, the Netherlands). 
D. Massera, M.K. Doris, S. Cadet, J. Kwiecinski, T.A. Pawade, F.E.C.M. Peeters, D. Dey, D.E. Newby, M.R. Dweck, P.J. Slomka.

Analytical quantification of aortic valve ${ }^{18} \mathrm{~F}$-sodium fluoride PET uptake.

Nucl Cardiol. 2018 Nov 29. doi: 10.1007/s12350-018-01542-6.

E.A.M.P Dudink, B.W. Florijn, B. Weijs, J.M.G.J. Duijs, J.G.L.M. Luermans, F.E.C.M. Peeters, L.J. Schurgers, J.E. Wildberger, U. Schotten, R. Bijkerk, H.J.G.M. Crijns, A.J. van Zonneveld.

Vascular calcification and not arrhythmia in idiopathic atrial fibrillation associates with sex differences in diabetic microvascular injury miRNA profiles.

Microrna. 2018 Nov 22. doi: 10.2174/2211536608666181122125208.

E.A.M.P. Dudink, F.E.C.M. Peeters, S. Altintas, L.I.B. Heckman, R.J. Haest, H. Kragten, B.L.J.H Kietselaer, J.E. Wildberger, J.G.L.M. Luermans, B. Weijs, H.J.G.M. Crijns.

The Agatston score of the descending aorta is an independent predictor of future coronary artery disease on top of coronary Agatston score in a low-risk population.

Open Heart 2018 Nov 10;5:e000893. doi:10.1136.

F.E.C.M. Peeters, E.A.M.P. Dudink, D.M. Kimenai, B. Weijs, S. Altintas, , L.I.B. Heckman, C. Mihl L.J. Schurgers, J.E. Wildberger, S.J.R. Meex, B.L.J.H. Kietselaer, H.J.G.M. Crijns.

Vitamin K Antagonists, Non-vitamin K Antagonist Oral AntiCoagulants and vascular calcification in patients with atrial fibrillation.

TH Open 2018 Sept 21:2:e391-e398.

\section{LIST OF PUBLICATIONS}

F.E.C.M. Peeters, M.J.W. van Mourik, S.J.R. Meex, J. Bucerius, S.M. Schalla, S.C. Gerretsen, C. Mihl M.R. Dweck, L.J. Schurgers, J.E. Wildberger, H.J.G.M. Crijns, B.L.J.H. Kietselaer.

Bicuspid Aortic valve Stenosis and the effect of vitamin K2 on calcification using ${ }^{18} \mathrm{~F}$-sodium fluoride positron emission tomography/magnetic resonance: the BASIK2 rationale and trial design.

Nutrients. 2018 Mar 21;10(4)

F.E.C.M. Peeters, S.J.R. Meex, M.R. Dweck, E. Aikawa, H.J.G.M. Crijns, L.J. Schurgers, B.L.J.H. Kietselaer. Calcific aortic valve stenosis: hard disease in the heart: A biomolecular approach towards diagnosis and treatment.

Eur Heart J. 2018 Jul 21:39(28):2618-2624

H.M.H. Spronk, T. Padro, J.E. Siland, J.H. Prochaska, J. Winters, A.C. van der Wal, J.J. Posthuma, G. Lowe, E. d'Alessandro, P. Wenzel, D.M. Coenen, P.H. Reitsma, W. Ruf, R.H. van Gorp, R.R. Koenen T. Vajen, N.A. Alshaikh, A.S. Wolberg, F.L. Macrae, N. Asquith, J. Heemskerk, A. Heinzmann, M. Moorlag, N. Mackman, P. van der Meijden, J.C.M. Meijers, M. Heestermans, T. Renné, S. Dólleman W. Chayouâ, R.A.S. Ariëns, C.C. Baaten, M. Nagy, A. Kuliopulos, J.J. Posma, P. Harrison, M.J. Vries, H.J.G.M. Crijns, E.A.M.P. Dudink, H.R. Buller, Y.M.C. Henskens, A. Själander, S. Zwaveling, O. Erküner, W. Eikelboom, A. Gulpen, F.E.C.M. Peeters, J. Douxfils, R.H. Olie, T. Baglin, A. Leader, U. Schotten, B. Scaf, H.M.M. van Beusekom, L.O. Mosnier, L. van der Vorm, P. Declerck, M. Visser, D.W.J. Dippel, V.J. Strijbis, K. Pertiwi, A.J. Ten Cate-Hoek, H. Ten Cate.

Atherothrombosis and thromboembolism: position paper from the second Maastricht Consensus Conference on Thrombosis.

Thromb. Haemost. 2018 Feb;118(2):229-250. 
N. van der Linden, T. Cornelis, D.M. Kimenai, L.J.J. Klinkenberg, J.M. Hilderink, S. Lück, E.J.R. Litjens, F.E.C.M. Peeters, A.S. Streng, T. Breidthardt, L.J.C. van Loon, O. Bekers, J.P. Kooman, P.O. Westermark, C. Mueller, S.J.R. Meex.

Origin of cardiac troponin Televations in chronic kidney disease.

Circulation. 2017 Sep 12;136(11):1073-1075

V.M. Brandenburg, S. Reinartz, N. Kaesler, T. Kruger, T. Dirrichs, R. Kramann, F.E.C.M. Peeters, J. Floege, A. Keszei, N. Marx, L.J. Schurgers, R. Koos.

Slower progress of aortic valve calcification with vitamin $\mathrm{K}$ supplementation: results from a prospective interventional proof-of-concept study.

Circulation. 2017. May 23; 135(21):2081-2083.

F.E.C.M. Peeters, N. van der Linden, A.L.L. Thomassen, H.J.G.M. Crijns, S.J.R. Meex, B.L.J.H. Kietselaer Clinical and echocardiographic determinants in bicuspid aortic dilatation.

Medicine (Baltimore). 2016 Dec;95(52):e5699.

F.E.C.M. Peeters, B.L.J.H. Kietselaer

Editorial to: Baseline MDCT findings after prosthetic heart valve implantation provide important complementary information to echocardiography for follow-up purposes by Suchá et al.

Eur Radiol. 2016 Apr; 26(4):1007-1008.

L.J.J. Klinkenberg, P. Luyten, N. van der Linden, K. Urgel, D.P.C. Snijders, C. Knackstedt, R. Dennert, B.L.J.H. Kietselaer, A.M.A. Mingels, E.P.M. Cardinaels, F.E.C.M. Peeters, J.D.E. van Suijlen, J. ten Kate, E. Marsch, T.L. Theelen, J.C. Sluimer, K. Wouters, O. Bekers, S.C.A.M. Bekkers, L.J.C. van Loon, M.P. van Dieijen-Visser, S.J.R. Meex.

Cardiac Troponin T and I Release after a 30-km Run.

Am J Cardiol. 2016 Jul 15;118(2):281-287.

B. Weijs, C.B. de Vos, R.G. Tieleman, F.E.C.M. Peeters, I. Limantoro, A.A. Kroon, E.C. Cheriex, R. Pisters, H.J.G.M. Crijns,

The occurrence of cardiovascular disease during 5-year follow-up in patients with idiopathic atrial fibrillation.

Europace. 2013. Jan;15(1): 18-23.

F.E.C.M. Peeters, E.A.M.P. Dudink, B. Weijs, L. Fabritz, W. Chua, B.L.J.H. Kietselaer, J.E. Wildberger, S.J.R. Meex, P. Kirchhof, H.J.G.M. Crijns, L.J. Schurgers.

Biomarkers associated with early aortic valve calcification: should we focus on gender specific processes? Submitted

F.E.C.M. Peeters, B.L.J.H. Kietselaer, J.M. Hilderink, N. van der Linden, M. Niens, H.J.G.M. Crijns, S.J.R. Meex. Variation of cardiac biomarkers in patients with aortic valve stenosis.

Submitted.

E.A.M.P Dudink, B. Weijs, J.G.L.M. Luermans. F.E.C.M. Peeters, S. Altintas, C.C.M.L.M. Timmermans, L.A.F.G. Pison, R.J. Haest, J.A. Kragten, B.L.J.H. Kietselaer, J.E. Wildberger, H.J.G.M. Crijns.

Computed tomography angiographic abnormalities predict incident cardiovascular disease in idiopathic AF - a 5 year follow-up study.

Submitted. 
